\title{
Enantioselective [2+2] Cycloadditions of Cinnamate Esters: Generalizing Lewis Acid Catalysis of Triplet Energy Transfer
}

Mary Elisabeth Daub, ${ }^{a}$ Hoimin Jung, ${ }^{b, c}$ Byung Joo Lee, ${ }^{a}$ Joonghee Won, ${ }^{b, c}$ Mu-Hyun Baik, $*, b, c$ and Tehshik P. Yoon*,a

${ }^{a}$ Department of Chemistry, University of Wisconsin-Madison, Madison, Wisconsin 53706, United States

${ }^{b}$ Department of Chemistry, Korea Advanced Institute of Science and Technology (KAIST), Daejeon 34141, South Korea

${ }^{c}$ Center for Catalytic Hydrocarbon Functionalizations, Institute for Basic Science (IBS), Daejeon 34141, Republic of Korea

mbaik2805@kaist.ac.krandtyoon@chem.wisc.edu

Supporting Information

\section{Table of Contents}

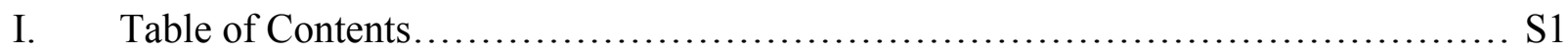

II. General Experimental Methods......................................... S2

III. Experimental Procedures and Characterization Data............................ S3

IV. Asymmetric Synthesis of Norlignan Natural Product 3 ......................... S29

V. HPLC Chromatograms............................................... S32

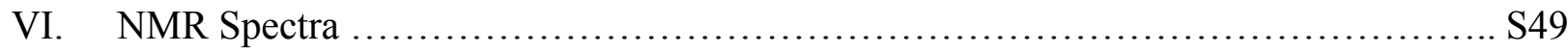

VII. Stern-Volmer Quenching Experiments...................................... 69

VIII. Emission Spectra of Iridium(III) Photocatalysts............................. S71

IX. Computational Details............................................... S72

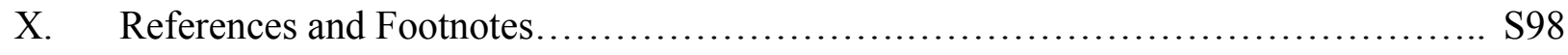




\section{General Experimental Methods}

Reagent Preparation. All reactions were performed under an inert atmosphere of nitrogen using oven-dried or flame-dried glassware and Teflon ${ }^{\circledR}$ coated stir bars. Solvents were dried by passage through columns of activated alumina. A 15W Eagle Light PAR38 blue LED flood light (500 lumens) or a Kessil H150 Blue LED light were used for all photochemical reactions as noted. All commercially available aldehydes and olefinic coupling partners were purified by distillation prior to use, and all other reagents were prepared using known literature procedures. Reactions were monitored by thin-layer chromatography (TLC) performed on $250 \mu \mathrm{m}$ silica gel 60 plates with $254 \mathrm{~nm}$ fluorescent indicator from EMD Chemicals using UV light as a visualizing agent and ceric ammonium molybdate and heat as developing agents. Flash chromatography was performed with Silicycle 40-63A silica (230-40 mesh).

Product Characterization. NMR spectra were recorded on a Bruker Avance-400, Avance-500, or Avance-600 MHz spectrometer with $\mathrm{DCH}$, Prodigy, BBFO+, or TCI-F probes. Chemical shifts are reported in parts per million and are internally referenced to tetramethyl silane $(0.00$ ppm) or the residual protio-solvent peak $\left(\mathrm{CDCl}_{3}: 7.26 \mathrm{ppm}\right.$ for ${ }^{1} \mathrm{H} \mathrm{NMR}$ and $77.16 \mathrm{ppm}$ for ${ }^{13} \mathrm{C}$ NMR). ${ }^{19} \mathrm{~F}\left\{{ }^{1} \mathrm{H}\right\}$ spectra were absolute referenced to the corresponding ${ }^{1} \mathrm{H}$ spectra using the method described by Harris. Data are reported as follows: chemical shift, multiplicity $($ ap $=$ apparent, $\mathrm{s}=$ singlet, $\mathrm{d}=$ doublet, $\mathrm{t}=$ triplet, $\mathrm{q}=$ quartet, $\mathrm{p}=$ pentet, $\mathrm{sext}=$ sextet, $\mathrm{sept}=$ septet, $\mathrm{m}$ $=$ multiplet, $\mathrm{br}=$ broad $)$, coupling constant(s) in Hz, integration. NMR spectra were obtained at $298 \mathrm{~K}$ unless otherwise noted. FT-IR spectra were recorded on a Bruker Alpha Platinum spectrometer and are reported in terms of frequency of absorption $\left(\mathrm{cm}^{-1}\right)$. Optical rotations were measured with a Rudolph Research Autopol III polarimeter operating on the sodium D-line (589 $\mathrm{nm}$ ) using a $50 \mathrm{~mm}$ path-length cell and are reported as: $[\alpha]_{\mathrm{D}}{ }^{\mathrm{T}}$ (concentration in $\mathrm{g} / 100 \mathrm{~mL}$, 
solvent). Analytical chiral HPLC was performed with a Waters Alliance system HPLC using Daicel CHIRALPAK ${ }^{\circledR}$ columns $(4.6 \mathrm{~mm} \times 25 \mathrm{~cm})$ and Chromasolv ${ }^{\circledR}$-grade $i$-PrOH and hexanes. High resolution mass spectra (HRMS) were recorded on a Thermo Q Exactive ${ }^{\mathrm{TM}}$ Plus using ESI-TOF (electrospray ionization-time of flight). Melting points (mp) are uncorrected and were measured on a Mel-Temp II melting point apparatus.

\section{Experimental Procedures and Characterization Data}

\section{General Procedure A: Synthesis of Cinnamic Esters by Acylation of Alcohols}

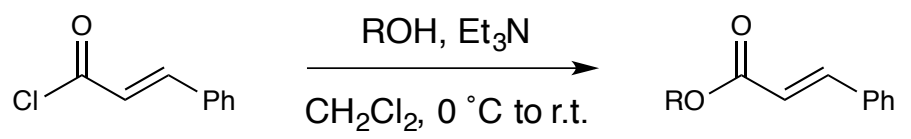

To a solution of cinnamoyl chloride (1 equiv) in $\mathrm{CH}_{2} \mathrm{Cl}_{2}(0.5 \mathrm{M})$ at $0{ }^{\circ} \mathrm{C}$ in an ice bath was added triethylamine (2.2 equiv) dropwise via syringe followed by the alcohol (1.1 equiv). After five minutes, the solution was allowed to warm to room temperature. After $17 \mathrm{~h}, 1 \mathrm{M} \mathrm{HCl}$ was added to the reaction mixture, and the aqueous layer was extracted three times with $\mathrm{CH}_{2} \mathrm{Cl}_{2}$. The combined organic extracts were washed with water, dried over $\mathrm{MgSO}_{4}$, filtered, and concentrated in vacuo. The crude material was purified by flash column chromatography, yielding the desired cinnamic ester.

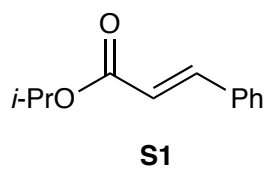

Isopropyl cinnamate (S1). The title compound was prepared according to General Procedure A using cinnamoyl chloride (1.00 g, $6.00 \mathrm{mmol})$, triethylamine $(1.84 \mathrm{~mL}, 13.20 \mathrm{mmol}), 2$-propanol (0.51 mL, $6.60 \mathrm{mmol})$, and $\mathrm{CH}_{2} \mathrm{Cl}_{2}(12 \mathrm{~mL})$. The crude material was purified by flash chromatography ( $\mathrm{SiO}_{2}, 3 \%$ EtOAc:hexanes) to afford isopropyl cinnamate $(611.0 \mathrm{mg}, 54 \%)$ as a colorless oil. The spectral data for this compound are consistent with those reported in the 
literature. ${ }^{1}{ }^{1} \mathrm{H} \mathrm{NMR}\left(\mathrm{CDCl}_{3}, 500 \mathrm{MHz}\right) \delta 7.67(\mathrm{~d}, J=16.0 \mathrm{~Hz}, 1 \mathrm{H}), 7.55-7.50(\mathrm{~m}, 2 \mathrm{H}), 7.41-$ $7.36(\mathrm{~m}, 3 \mathrm{H}), 6.42(\mathrm{~d}, J=16.0 \mathrm{~Hz}, 1 \mathrm{H}), 5.14(\mathrm{sept}, J=6.3 \mathrm{~Hz}, 1 \mathrm{H}), 1.32(\mathrm{~d}, J=6.4 \mathrm{~Hz}, 6 \mathrm{H}),{ }^{13} \mathrm{C}$ NMR (126 MHz, $\left.\mathrm{CDCl}_{3}\right) \delta 166.5,144.3,134.5,130.1,128.0,118.8,67.8,22.0$.

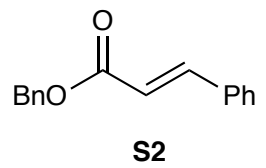

Benzyl cinnamate (S2). The title compound was prepared according to General Procedure A using cinnamoyl chloride (0.956 g, $5.74 \mathrm{mmol})$, triethylamine $(1.76 \mathrm{~mL}, 12.62 \mathrm{mmol})$, benzyl alcohol $(0.65 \mathrm{~mL}, 6.31 \mathrm{mmol})$, and $\mathrm{CH}_{2} \mathrm{Cl}_{2}(11.5 \mathrm{~mL})$. The crude material was purified by flash chromatography $\left(\mathrm{SiO}_{2}, 3 \%\right.$ EtOAc:hexanes) to afford benzyl cinnamate $(1.146 \mathrm{~g}, 84 \%)$ as a white solid. The spectral data for this compound are consistent with those reported in the literature. ${ }^{1}{ }^{1} \mathrm{H}$ NMR $\left(\mathrm{CDCl}_{3}, 400 \mathrm{MHz}\right) \delta 7.73(\mathrm{~d}, J=16.0 \mathrm{~Hz}, 1 \mathrm{H}), 7.56-7.49(\mathrm{~m}, 2 \mathrm{H}), 7.45-$ $7.31(\mathrm{~m}, 8 \mathrm{H}), 6.49(\mathrm{~d}, J=16.0 \mathrm{~Hz}, 1 \mathrm{H}), 5.26(\mathrm{~s}, 2 \mathrm{H}) ;{ }^{13} \mathrm{C} \mathrm{NMR}\left(126 \mathrm{MHz}, \mathrm{CDCl}_{3}\right) \delta 166.8$ 145.2, 136.1, 136.0, 134.3, 130.4, 128.9, 128.6, 128.29, 128.26, 128.1, 117.9, 66.4.

\section{General Procedure B: Synthesis of Cinnamic Esters by Wittig Olefination}

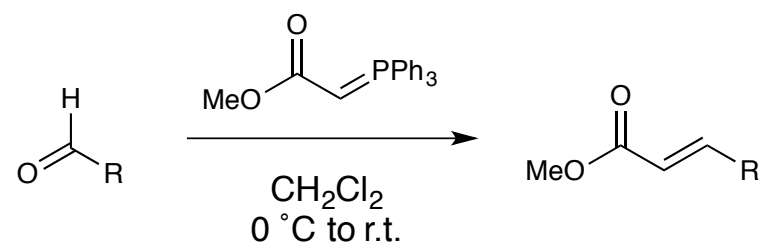

To a solution of freshly distilled aldehyde (1 equiv) in $\mathrm{CH}_{2} \mathrm{Cl}_{2}(0.2 \mathrm{M})$ at $0{ }^{\circ} \mathrm{C}$ in an ice bath was added methyl (triphenylphosphoranylidene)acetate (1.5 equiv) in one portion. After thirty minutes, the reaction mixture was allowed to warm to room temperature. After $19 \mathrm{~h}$, the solvent was removed in vacuo, and the crude residue was purified by flash column chromatography, yielding the desired cinnamic ester. 


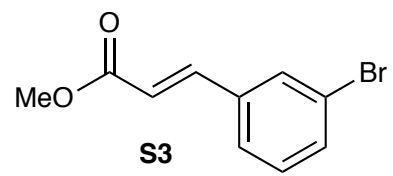

Methyl (E)-3-(3-bromophenyl)acrylate (S3). The title compound was prepared according to General Procedure B using 3-bromobenzaldehyde (0.64 mL, 5.49 mmol), methyl (triphenylphosphoranylidene)acetate $(2.71 \mathrm{~g}, 8.11 \mathrm{mmol})$, and $\mathrm{CH}_{2} \mathrm{Cl}_{2}(27 \mathrm{~mL})$. The crude material was purified by flash chromatography $\left(\mathrm{SiO}_{2}, 5 \%\right.$ EtOAc:hexanes) to afford methyl $(E)$ 3-(3-bromophenyl)acrylate (1.25 g, 96\%) as a white solid. The spectral data for this compound are consistent with those reported in the literature. ${ }^{2}{ }^{1} \mathrm{H} \mathrm{NMR}\left(\mathrm{CDCl}_{3}, 500 \mathrm{MHz}\right) \delta 7.67(\mathrm{~s}, 1 \mathrm{H})$, $7.61(\mathrm{~d}, J=16.0 \mathrm{~Hz}, 1 \mathrm{H}), 7.51(\mathrm{~d}, J=8.0 \mathrm{~Hz}, 1 \mathrm{H}), 7.44(\mathrm{~d}, J=8.0 \mathrm{~Hz}, 1 \mathrm{H}), 7.26(\mathrm{t}, J=7.9 \mathrm{~Hz}$, $1 \mathrm{H}), 6.43(\mathrm{~d}, J=16.0 \mathrm{~Hz}, 1 \mathrm{H}), 3.81(\mathrm{~s}, 3 \mathrm{H}) ;{ }^{13} \mathrm{C} \mathrm{NMR}\left(126 \mathrm{MHz}, \mathrm{CDCl}_{3}\right) \delta 167.0,143.1,136.5$, $133.1,130.7,130.4,126.7,123.0,119.3,51.9$.

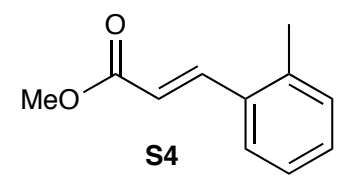

Methyl (E)-3-(o-tolyl)acrylate (S4). The title compound was prepared according to General Procedure B using $\quad$-tolualdehyde $\quad(0.69 \quad \mathrm{~mL}, \quad 5.97 \quad \mathrm{mmol})$, methyl (triphenylphosphoranylidene)acetate $(3.01 \mathrm{~g}, 9.00 \mathrm{mmol})$, and $\mathrm{CH}_{2} \mathrm{Cl}_{2}(30 \mathrm{~mL})$. The crude material was purified by flash chromatography $\left(\mathrm{SiO}_{2}, 5 \%\right.$ EtOAc:hexanes) to afford methyl $(E)-$ 3-(o-tolyl)acrylate $(823 \mathrm{mg}, 78 \%)$ as a colorless oil. The spectral data for this compound are consistent with those reported in the literature. ${ }^{3} \mathrm{H}$ NMR $\left(\mathrm{CDCl}_{3}, 500 \mathrm{MHz}\right) \delta 7.98(\mathrm{~d}, J=15.9$ $\mathrm{Hz}, 1 \mathrm{H}), 7.55(\mathrm{~d}, J=7.2 \mathrm{~Hz}, 1 \mathrm{H}), 7.31-7.24(\mathrm{~m}, 1 \mathrm{H}), 7.23-7.18(\mathrm{~m}, 1 \mathrm{H}), 6.36(\mathrm{~d}, J=15.9 \mathrm{~Hz}$, 1H), $3.81(\mathrm{~s}, 3 \mathrm{H}), 2.44(\mathrm{~s}, 3 \mathrm{H})$; ${ }^{\mathrm{B}} \mathrm{C} \mathrm{NMR}\left(126 \mathrm{MHz}, \mathrm{CDCl}_{3}\right) \delta 167.5,142.5,137.7,133.4,130.8$, $130.0,126.4,126.3,118.8,51.7,19.8$. 


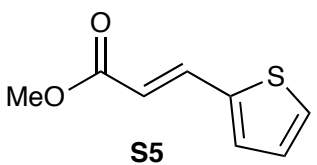

Methyl (E)-3-(thiophen-2-yl)acrylate (S5). The title compound was prepared according to General Procedure B using 2-thiophenecarboxaldehyde $(0.56 \mathrm{~mL}, 6.00 \mathrm{mmol})$, methyl (triphenylphosphoranylidene)acetate $(3.01 \mathrm{~g}, 9.00 \mathrm{mmol})$, and $\mathrm{CH}_{2} \mathrm{Cl}_{2}(30 \mathrm{~mL})$. The crude material was purified by flash chromatography $\left(\mathrm{SiO}_{2}, 5 \%\right.$ EtOAc:hexanes) to afford methyl $(E)$ 3-(thiophen-2-yl)acrylate $(951 \mathrm{mg}, 94 \%)$ as a white solid. The spectral data for this compound are consistent with those reported in the literature. ${ }^{4} \mathrm{H}$ NMR $\left(\mathrm{CDCl}_{3}, 500 \mathrm{MHz}\right) \delta 7.79(\mathrm{~d}, J=$ $15.7 \mathrm{~Hz}, 1 \mathrm{H}), 7.37$ (d, $J=5.0 \mathrm{~Hz}, 1 \mathrm{H}), 7.25(\mathrm{~d}, J=3.6 \mathrm{~Hz}, 1 \mathrm{H}), 7.05(\mathrm{dd}, J=5.1,3.6 \mathrm{~Hz}, 1 \mathrm{H})$, $6.24(\mathrm{~d}, J=15.7 \mathrm{~Hz}, 1 \mathrm{H}), 3.79(\mathrm{~s}, 3 \mathrm{H}) ;{ }^{13} \mathrm{C} \mathrm{NMR}\left(126 \mathrm{MHz}, \mathrm{CDCl}_{3}\right) \delta 167.3,139.5,137.3$, $130.9,128.4,128.1,116.5,51.7$.
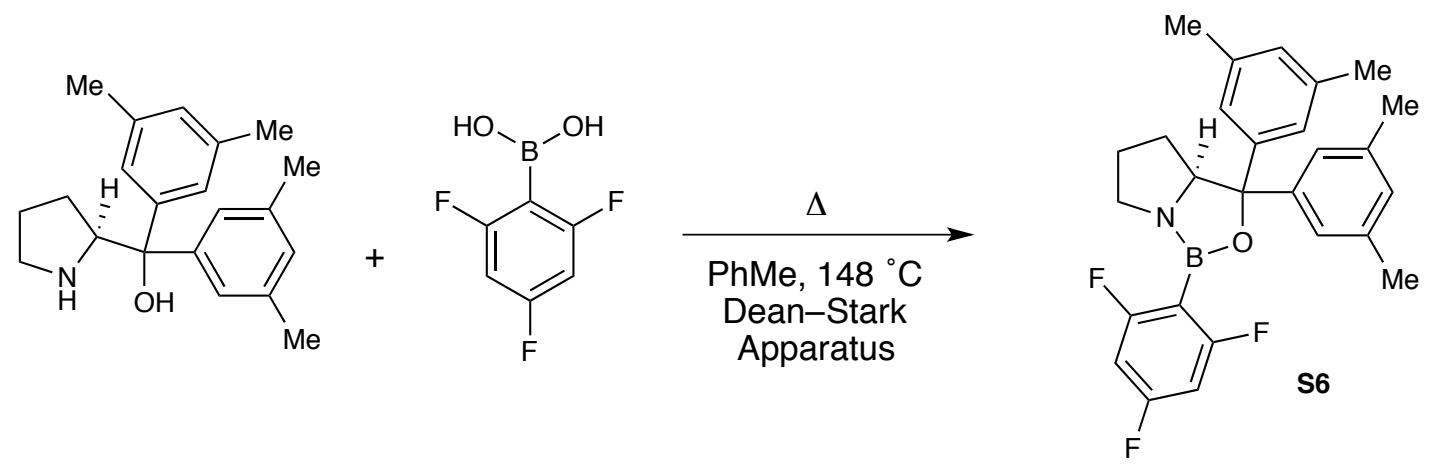

Oxazaborolidine Precursor S6. The following procedure was adapted from Bach et al. ${ }^{5}$ A 25 $\mathrm{mL}$ round-bottom flask was charged with 2,4,6-trifluorophenylboronic acid (1.00 equiv) and equipped with a Dean-Stark apparatus. The apparatus was purged with $\mathrm{N}_{2}$ and a solution of $(S)$ bis(3,5-dimethylphenyl)(pyrrolidin-2-yl)methanol (1.00 equiv, $0.1 \mathrm{M}$ in $\mathrm{PhMe}$ ) was added to the $25 \mathrm{~mL}$ round-bottom flask. Additional toluene was added to the reaction mixture to bring the concentration to $0.02 \mathrm{M}$, and the Dean-Stark apparatus was filled with toluene. The reaction mixture was heated to reflux (Bath Temperature: $148{ }^{\circ} \mathrm{C}$ ). After three hours, the Dean-Stark trap 
was drained and half of the toluene in the reaction vessel was removed by distillation. The DeanStark apparatus was refilled with toluene, and the volume of toluene that was distilled from the reaction mixture was replenished. The procedure was repeated again after refluxing for 3 hours. After 16 hours, the toluene was distilled from the reaction mixture to a volume of $\sim 1 \mathrm{~mL}$. After allowing to cool to room temperature, the Dean-Stark apparatus was quickly removed under flow of nitrogen and replaced with a septum, and the remaining toluene was removed in vacuo. The oxazaborolidine was dissolved in $\mathrm{CH}_{2} \mathrm{Cl}_{2}(0.15 \mathrm{M})$ and used directly. The oxazaborolidine was freshly prepared for each photochemical reaction. ${ }^{1} \mathrm{H} \mathrm{NMR}\left(\mathrm{CDCl}_{3}, 500 \mathrm{MHz}\right) \delta 7.10(\mathrm{~s}$, 2H), $6.98(\mathrm{~s}, 2 \mathrm{H}), 6.80(\mathrm{~s}, 1 \mathrm{H}), 6.76(\mathrm{~s}, 1 \mathrm{H}), 6.56(\mathrm{dd}, J=9.1,7.2 \mathrm{~Hz}, 2 \mathrm{H}), 4.47(\mathrm{dd}, J=10.1$, $5.6 \mathrm{~Hz}, 1 \mathrm{H}), 3.21(\mathrm{dt}, J=10.6,7.3 \mathrm{~Hz}, 1 \mathrm{H}), 3.08(\mathrm{ddd}, J=10.8,91,5.2 \mathrm{~Hz}, 1 \mathrm{H}), 2.22(\mathrm{~s}, 6 \mathrm{H})$, $2.20(\mathrm{~s}, 6 \mathrm{H}), 1.80-1.69(\mathrm{~m}, 2 \mathrm{H}), 1.66-1.58(\mathrm{~m}, 1 \mathrm{H}), 0.85(\mathrm{dq}, J=12.2,9.8 \mathrm{~Hz}, 1 \mathrm{H}) ;{ }^{13} \mathrm{C} \mathrm{NMR}$ $\left(126 \mathrm{MHz}, \mathrm{CDCl}_{3}\right) \delta 165.5(\mathrm{ddd}, J=249.5,16.8,15.0 \mathrm{~Hz}), 163.5(\mathrm{dt}, J=250.5,15.8 \mathrm{~Hz}), 146.1$, 142.4, 136.5, 136.0, 127.9, 127.3, 123.1, 123.0, 103.4-102.2 (m), ${ }^{6} 99.1-98.7$ (m), 87.7, 72.3, 42.0, 29.5, 25.4, 20.54, 20.49; ${ }^{19} \mathrm{~F}$ NMR $\left(376 \mathrm{MHz}, \mathrm{CDCl}_{3}\right) \delta-96.9(\mathrm{~d}, J=8.3 \mathrm{~Hz}, 2 \mathrm{~F}),-105.8$ $(\mathrm{t}, J=8.3 \mathrm{~Hz}, 1 \mathrm{~F}) ;{ }^{11} \mathrm{~B} \mathrm{NMR}\left(128 \mathrm{MHz}, \mathrm{CDCl}_{3}\right) \delta 29.8($ br s).
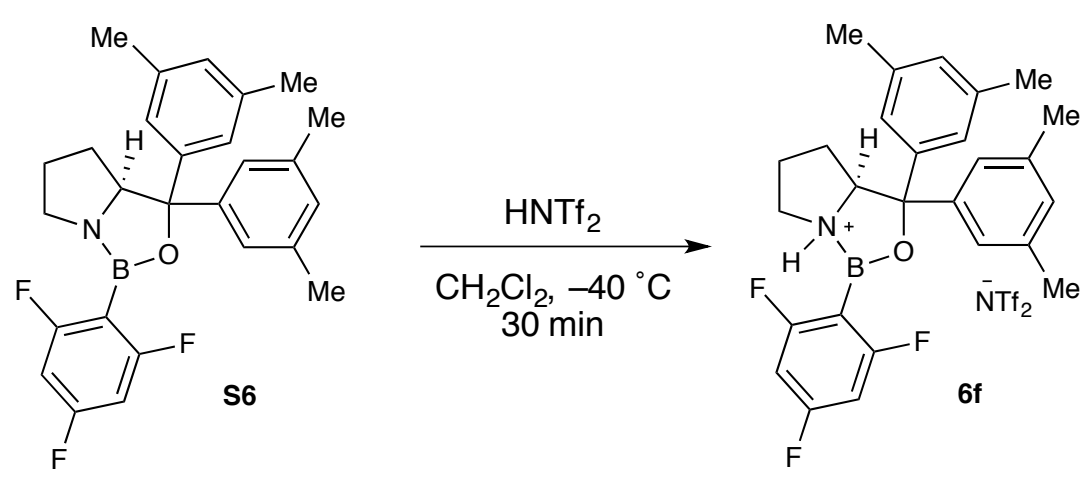

Oxazaborolidine 6f. A solution of oxazaborolidine precursor $\mathbf{S 6}$ (1.2 equiv, $0.15 \mathrm{M}$ in $\mathrm{CH}_{2} \mathrm{Cl}_{2}$ ) was cooled to $-40{ }^{\circ} \mathrm{C}$. A solution of trifluoromethanesulfonimide (1.0 equiv, $0.5 \mathrm{M}$ solution in 
$\mathrm{CH}_{2} \mathrm{Cl}_{2}$ ) was added dropwise via syringe. After $30 \mathrm{~min}$ at $-40{ }^{\circ} \mathrm{C}$, oxazaborolidine $\mathbf{6 f}$ was used directly in the enantioselective [2+2] photocycloaddition.

\section{General Procedure C: Enantioselective [2+2] Photocycloaddition of Cinnamic Esters}

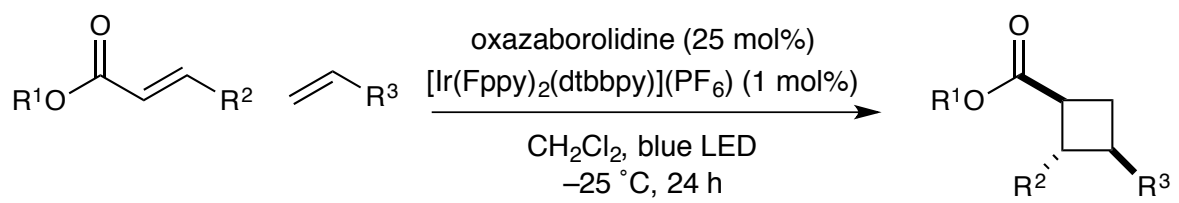

(5 equiv)

To a solution of cinnamic ester (1 equiv) and photocatalyst $(1 \mathrm{~mol} \%)$ in $\mathrm{CH}_{2} \mathrm{Cl}_{2}(0.032 \mathrm{M})$ cooled to $-40{ }^{\circ} \mathrm{C}$ was added a pre-cooled solution of oxazaborolidine $6 \mathbf{f}(25 \mathrm{~mol} \%, 0.1 \mathrm{M}$ in $\mathrm{CH}_{2} \mathrm{Cl}_{2}$ ) dropwise via syringe. After five minutes, styrene (5 equiv) was added dropwise via syringe. The reaction mixture was allowed to warm to $-25{ }^{\circ} \mathrm{C}$, and irradiated with a HG150 Kessil lamp at a distance of $6 \mathrm{~cm}$. After $24 \mathrm{~h}$, the reaction was quenched with triethylamine $(0.1$ $\mathrm{mL}$ ). The reaction mixture was allowed to warm to room temperature and filtered through a pad of silica (elution with $\mathrm{Et}_{2} \mathrm{O}$ ). The filtrate was concentrated in vacuo, and the crude residue was purified using flash chromatography, yielding the desired [2+2] cycloadduct.

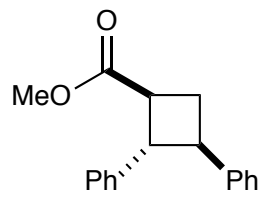

5

Methyl (1S,2S,3R)-2,3-diphenylcyclobutane-1-carboxylate (5). The title compound was prepared according to General Procedure C using methyl cinnamate (64.9 $\mathrm{mg}, 0.400 \mathrm{mmol}$ ), $\left[\operatorname{Ir}(\mathrm{Fppy})_{2}(\mathrm{dtbpy})\left(\mathrm{PF}_{6}\right)\right](3.8 \mathrm{mg}, 0.004 \mathrm{mmol})$, styrene $(0.23 \mathrm{~mL}, 2.00 \mathrm{mmol})$, oxazaborolidine 6f $(1.0 \mathrm{~mL}, 0.100 \mathrm{mmol})$, and $\mathrm{CH}_{2} \mathrm{Cl}_{2}(12.5 \mathrm{~mL})$. The crude material resulted in a $6: 1$ mixture of diastereomers that was purified by flash chromatography $\left(\mathrm{SiO}_{2}\right.$, Column 1: 2.5\% MTBE:pentane, Column 2: 3\% $\mathrm{Et}_{2} \mathrm{O}$ :pentane). Combined yield: $97.3 \mathrm{mg}$ of a clear oil (91\%, $\left.0.365 \mathrm{mmol}\right) ; 97 \%$ 
ee (Daicel CHIRALPAK ${ }^{\circledR}$ OD-H, 5-50\% IPA:hexanes over $13 \mathrm{~min}$, flow rate of $1 \mathrm{~mL} / \mathrm{min} ; \mathrm{t}_{1}=$ $\left.5.20 \mathrm{~min}, \mathrm{t}_{2}=6.62 \mathrm{~min}\right) .[\alpha]_{\mathrm{D}}{ }^{20}=-39.8^{\circ}\left(\mathrm{c}=1.0, \mathrm{CH}_{2} \mathrm{Cl}_{2}\right) ;{ }^{1} \mathrm{H} \mathrm{NMR}\left(\mathrm{CDCl}_{3}, 500 \mathrm{MHz}\right) \delta 7.37-$ $7.18(\mathrm{~m}, 10 \mathrm{H}), 3.85(\mathrm{t}, J=9.7 \mathrm{~Hz}, 1 \mathrm{H}), 3.72(\mathrm{~s}, 3 \mathrm{H}), 3.53(\mathrm{td}, J=10,8.3 \mathrm{~Hz}, 1 \mathrm{H}), 3.20(\mathrm{dt}, J=$ 9.7, $8.3 \mathrm{~Hz}, 1 \mathrm{H}), 2.63(\mathrm{dt}, J=10.6,8.3 \mathrm{~Hz}, 1 \mathrm{H}), 2.41$ (q, $J=10.3 \mathrm{~Hz}, 1 \mathrm{H}) ;{ }^{13} \mathrm{C} \mathrm{NMR}(126 \mathrm{MHz}$, $\left.\mathrm{CDCl}_{3}\right) \delta 174.6,143.2,142.1,128.47,128.46,126.8,126.7,126.6,126.57,51.9,50.8,43.5,41.5$, 29.6; IR (thin film) v 3059, 3027, 2987, 2948, 1728, 1600, 1496, 1439, 1200, 1159, 1027, 751, $696 \mathrm{~cm}^{-1}$; HRMS (ESI) $\mathrm{m} / \mathrm{z}$ calcd for $\mathrm{C}_{18} \mathrm{H}_{18} \mathrm{O}_{2} \mathrm{Na}(\mathrm{M}+\mathrm{Na})^{+} 289.1199$, found 289.1194. Selective ${ }^{1}$ H-NOESY-1D spectra were obtained to determine the relative stereochemistry. Selected NOE interactions are shown.
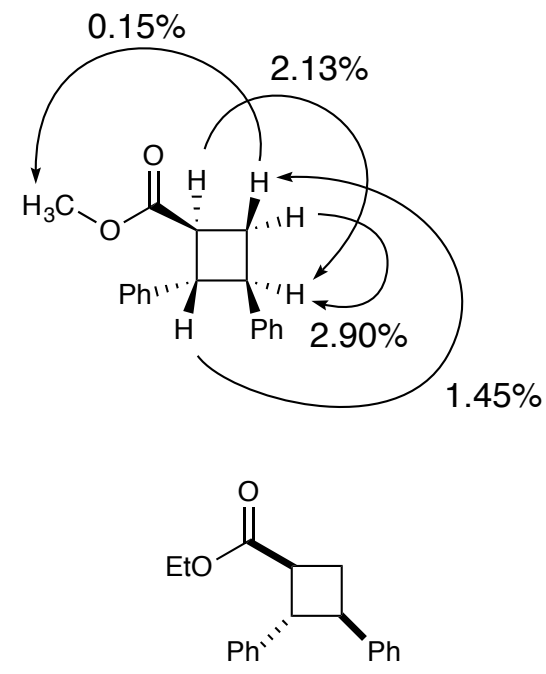

8

Ethyl (1S,2S,3R)-2,3-diphenylcyclobutane-1-carboxylate (8). The title compound was prepared according to General Procedure C using ethyl cinnamate $(70.5 \mathrm{mg}, 0.400 \mathrm{mmol}$ ), $\left[\operatorname{Ir}(\mathrm{Fppy})_{2}(\mathrm{dtbpy})\left(\mathrm{PF}_{6}\right)\right](3.8 \mathrm{mg}, 0.004 \mathrm{mmol})$, styrene $(0.23 \mathrm{~mL}, 2.00 \mathrm{mmol})$, oxazaborolidine 6f $(1.0 \mathrm{~mL}, 0.100 \mathrm{mmol})$, and $\mathrm{CH}_{2} \mathrm{Cl}_{2}(12.5 \mathrm{~mL})$. The crude material resulted in a $6: 1$ mixture of diastereomers that was purified by flash chromatography $\left(\mathrm{SiO}_{2}\right.$, Column 1: 4\% EtOAc:hexanes, Column 2: $4 \% \mathrm{MTBE} / \mathrm{pentane})$. Combined yield: $105.3 \mathrm{mg}$ of a clear oil $(94 \%, 0.376 \mathrm{mmol})$; 
98\% ee (Daicel CHIRALPAK $®$ OD-H, 5-50\% IPA:hexanes over $13 \mathrm{~min}$, flow rate of 1 $\left.\mathrm{mL} / \mathrm{min} ; \mathrm{t}_{1}=4.89 \mathrm{~min}, \mathrm{t}_{2}=6.09 \mathrm{~min}\right) .[\alpha]_{\mathrm{D}}{ }^{20}=-41.8^{\circ}\left(\mathrm{c}=1.0, \mathrm{CH}_{2} \mathrm{Cl}_{2}\right) ;{ }^{1} \mathrm{H} \mathrm{NMR}\left(\mathrm{CDCl}_{3}, 500\right.$ MHz) $\delta 7.34-7.18(\mathrm{~m}, 10 \mathrm{H}), 4.18(\mathrm{q}, J=7.2 \mathrm{~Hz}, 2 \mathrm{H}), 3.85(\mathrm{t}, J=9.8 \mathrm{~Hz}, 1 \mathrm{H}), 3.55(\mathrm{td}, J=9.8$, $8.3 \mathrm{~Hz}, 1 \mathrm{H}), 3.17$ (ap q, $J=9.3 \mathrm{~Hz}, 1 \mathrm{H}), 2.63(\mathrm{dt}, J=10.6,8.3 \mathrm{~Hz}, 1 \mathrm{H}), 2.40$ (q, $J=10.3 \mathrm{~Hz}$, 1H), $1.28(\mathrm{t}, J=7.2 \mathrm{~Hz}, 3 \mathrm{H}) ;{ }^{13} \mathrm{C}$ NMR $\left(126 \mathrm{MHz}, \mathrm{CDCl}_{3}\right) \delta 174.1,143.3,142.2,128.4(2 \mathrm{C})$, $126.8,126.63,126.62,126.5,60.6,50.7,43.3,41.8,29.6,14.3$; IR (thin film) $v$ 3060, 3028, 2980, 2903, 1726, 1603, 1496, 1446, 1195, 1161, 1029, $752 \mathrm{~cm}^{-1}$; HRMS (ESI) $\mathrm{m} / \mathrm{z}$ calcd for $\mathrm{C}_{19} \mathrm{H}_{20} \mathrm{O}_{2} \mathrm{Na}(\mathrm{M}+\mathrm{Na})^{+}$303.1355, found 303.1352.

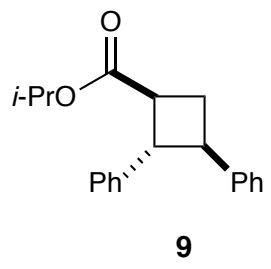

Isopropyl $(1 S, 2 S, 3 R)$-2,3-diphenylcyclobutane-1-carboxylate (9). The title compound was prepared according to General Procedure C using isopropyl cinnamate (76.1 $\mathrm{mg}, 0.400 \mathrm{mmol}$ ), $\left[\operatorname{Ir}(\mathrm{Fppy})_{2}(\mathrm{dtbpy})\left(\mathrm{PF}_{6}\right)\right](3.8 \mathrm{mg}, 0.004 \mathrm{mmol})$, styrene $(0.23 \mathrm{~mL}, 2.00 \mathrm{mmol})$, oxazaborolidine 6f $(1.0 \mathrm{~mL}, 0.100 \mathrm{mmol})$, and $\mathrm{CH}_{2} \mathrm{Cl}_{2}(12.5 \mathrm{~mL})$. The crude material resulted in a 7:1 mixture of diastereomers that was purified by flash chromatography $\left(\mathrm{SiO}_{2}\right.$, Column 1: 4\% EtOAc:hexanes, Column 2: 4\% MTBE:pentane). Combined yield: $112.9 \mathrm{mg}$ of a clear oil (96\%, $0.384 \mathrm{mmol})$; 97\% ee (Daicel CHIRALPAK® OD-H, 5-50\% IPA:hexanes over 13 min, flow rate of 1 $\left.\mathrm{mL} / \mathrm{min} ; \mathrm{t}_{1}=4.37 \mathrm{~min}, \mathrm{t}_{2}=4.86 \mathrm{~min}\right) .[\alpha]_{\mathrm{D}}{ }^{20}=-41.6^{\circ}\left(\mathrm{c}=1.0, \mathrm{CH}_{2} \mathrm{Cl}_{2}\right) ;{ }^{1} \mathrm{H} \mathrm{NMR}\left(\mathrm{CDCl}_{3}, 500\right.$ $\mathrm{MHz}) \delta 7.34-7.18(\mathrm{~m}, 10 \mathrm{H}), 5.06(\mathrm{sept}, J=6.3 \mathrm{~Hz}, 1 \mathrm{H}), 3.84(\mathrm{t}, J=9.7 \mathrm{~Hz}, 1 \mathrm{H}), 3.55(\mathrm{td}, J=$ $10.0,8.3 \mathrm{~Hz}, 1 \mathrm{H}), 3.12(\mathrm{td}, J=9.7,8.5 \mathrm{~Hz}, 1 \mathrm{H}), 2.62(\mathrm{dt}, J=10.3,8.3 \mathrm{~Hz}, 1 \mathrm{H}), 2.38(\mathrm{q}, J=10.3$ $\mathrm{Hz}, 1 \mathrm{H}), 1.26(\mathrm{~d}, J=6.3 \mathrm{~Hz}, 3 \mathrm{H}) 1.25(\mathrm{~d}, J=6.3 \mathrm{~Hz}, 3 \mathrm{H}) ;{ }^{13} \mathrm{C} \mathrm{NMR}\left(126 \mathrm{MHz}, \mathrm{CDCl}_{3}\right) \delta 173.7$, $143.4,142.3,128.44,128.41,126.8,126.61,126.58,126.5,67.9,50.6,43.0,42.1,29.6,21.87$, 
21.85; IR (thin film) v 3061, 3027, 2979, 2943, 1722, 1603, 1495, 1448, 1197, 1106, 751, 696 $\mathrm{cm}^{-1}$; HRMS (ESI) $\mathrm{m} / \mathrm{z}$ calcd for $\mathrm{C}_{20} \mathrm{H}_{22} \mathrm{O}_{2} \mathrm{Na}(\mathrm{M}+\mathrm{Na})^{+}$317.1512, found 317.1509.

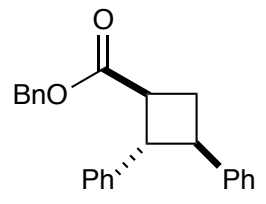

10

Benzyl (1S,2S,3R)-2,3-diphenylcyclobutane-1-carboxylate (10). The title compound was prepared according to General Procedure $\mathrm{C}$ using benzyl cinnamate $(95.3 \mathrm{mg}, 0.400 \mathrm{mmol}$ ), $\left[\operatorname{Ir}(\mathrm{Fppy})_{2}(\mathrm{dtbpy})\left(\mathrm{PF}_{6}\right)\right](3.8 \mathrm{mg}, 0.004 \mathrm{mmol})$, styrene $(0.23 \mathrm{~mL}, 2.00 \mathrm{mmol})$, oxazaborolidine 6f $(1.0 \mathrm{~mL}, 0.100 \mathrm{mmol})$, and $\mathrm{CH}_{2} \mathrm{Cl}_{2}(12.5 \mathrm{~mL})$. The crude material resulted in a $6: 1$ mixture of diastereomers that was purified by flash chromatography $\left(\mathrm{SiO}_{2}\right.$, Column 1: 4\% EtOAc:hexanes, Column 2: 4\% MTBE:pentane). Combined yield: $118.1 \mathrm{mg}$ of a clear oil $(86 \%, 0.345 \mathrm{mmol})$; 97\% ee (Daicel CHIRALPAK® OD-H, 5-50\% IPA:hexanes over $13 \mathrm{~min}$, flow rate of 1 $\left.\mathrm{mL} / \mathrm{min} ; \mathrm{t}_{1}=5.97 \mathrm{~min}, \mathrm{t}_{2}=6.50 \mathrm{~min}\right) .[\alpha]_{\mathrm{D}}{ }^{20}=-26.0^{\circ}\left(\mathrm{c}=1.0, \mathrm{CH}_{2} \mathrm{Cl}_{2}\right) ;{ }^{1} \mathrm{H} \mathrm{NMR}\left(\mathrm{CDCl}_{3}, 500\right.$ MHz) $\delta 7.39-7.18(\mathrm{~m}, 15 \mathrm{H}), 5.19(\mathrm{~d}, J=12.5 \mathrm{~Hz}, 1 \mathrm{H}), 5.15(\mathrm{~d}, J=12.5 \mathrm{~Hz}, 1 \mathrm{H}), 3.86(\mathrm{t}, J=9.8$ $\mathrm{Hz}, 1 \mathrm{H}), 3.56(\mathrm{q}, J=9.5 \mathrm{~Hz}, 1 \mathrm{H}), 3.24(\mathrm{q}, J=9.3 \mathrm{~Hz}, 1 \mathrm{H}), 2.64(\mathrm{dt}, J=10.7,8.3 \mathrm{~Hz}, 1 \mathrm{H}), 2.42$ $(\mathrm{q}, J=10.4 \mathrm{~Hz}, 1 \mathrm{H}) ;{ }^{13} \mathrm{C} \mathrm{NMR}\left(126 \mathrm{MHz}, \mathrm{CDCl}_{3}\right) \delta 173.9,143.1,142.0,136.0,128.54,128.45$ (2C), 128.2, 128.1, 126.8, 126.7, 126.66, 126.6, 66.4, 50.8, 43.3, 41.8, 29.5; IR (thin film) $v$ 3061, 3027, 2979, 2943, 1722, 1603, 1495, 1448, 1197, 1106, 751, $696 \mathrm{~cm}^{-1}$; HRMS (ESI) $\mathrm{m} / \mathrm{z}$ calcd for $\mathrm{C}_{20} \mathrm{H}_{22} \mathrm{O}_{2} \mathrm{Na}(\mathrm{M}+\mathrm{Na})^{+}$317.1512, found 317.1509.

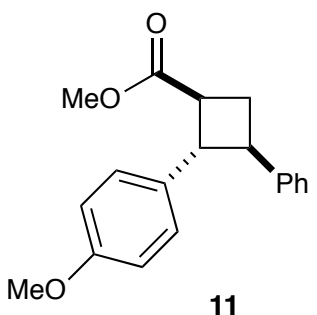


Methyl (1S,2S,3R)-2-(4-methoxyphenyl)-3-phenylcyclobutane-1-carboxylate (11). The title compound was prepared according to General Procedure C using methyl (E)-3-(4methoxyphenyl)acrylate ${ }^{7}(76.9 \mathrm{mg}, 0.400 \mathrm{mmol}),\left[\operatorname{Ir}(\mathrm{Fppy})_{2}(\mathrm{dtbpy})\left(\mathrm{PF}_{6}\right)\right](3.8 \mathrm{mg}, 0.004 \mathrm{mmol})$, styrene $(0.23 \mathrm{~mL}, 2.00 \mathrm{mmol})$, oxazaborolidine $\mathbf{6 f}(1.0 \mathrm{~mL}, 0.100 \mathrm{mmol})$, and $\mathrm{CH}_{2} \mathrm{Cl}_{2}(12.5 \mathrm{~mL})$. The crude material resulted in a single diastereomer $(>20: 1)$ that was purified by flash chromatography $\left(\mathrm{SiO}_{2}\right.$, Column 1: 8\% EtOAc:hexanes, Column 2: 10\% $\mathrm{Et}_{2} \mathrm{O}$ :pentane). Yield: $90.4 \mathrm{mg}$ of a clear oil $(76 \%, 0.305 \mathrm{mmol}) ; 94 \%$ ee (Daicel CHIRALPAK ${ }^{\circledR}$ OD-H, 5-50\% IPA:hexanes over $13 \mathrm{~min}$, flow rate of $\left.1 \mathrm{~mL} / \mathrm{min} ; \mathrm{t}_{1}=6.16 \mathrm{~min}, \mathrm{t}_{2}=7.69 \mathrm{~min}\right) .[\alpha]_{\mathrm{D}}{ }^{20}=-46.4^{\circ}$ $\left(\mathrm{c}=1.0, \mathrm{CH}_{2} \mathrm{Cl}_{2}\right) ;{ }^{1} \mathrm{H} \mathrm{NMR}\left(\mathrm{CDCl}_{3}, 500 \mathrm{MHz}\right) \delta 7.33-7.28(\mathrm{~m}, 2 \mathrm{H}), 7.27-7.23(\mathrm{~m}, 2 \mathrm{H}), 7.23-$ $7.19(\mathrm{~m}, 1 \mathrm{H}), 7.18(\operatorname{ap~d}, J=8.5 \mathrm{~Hz}, 2 \mathrm{H}), 6.84(\operatorname{ap~d}, J=8.6 \mathrm{~Hz}, 2 \mathrm{H}), 3.78(\mathrm{~s}, 3 \mathrm{H}), 3.75(\mathrm{t}, J=$ $9.8 \mathrm{~Hz}, 1 \mathrm{H}), 3.71(\mathrm{~s}, 3 \mathrm{H}), 3.49(\mathrm{dt}, J=10.0,8.3 \mathrm{~Hz}, 1 \mathrm{H}), 3.15(\mathrm{dt}, J=9.6,8.3 \mathrm{~Hz}, 1 \mathrm{H}), 2.60(\mathrm{td}$, $J=10.5,8.3 \mathrm{~Hz}, 1 \mathrm{H}), 2.39(\mathrm{q}, J=10.5 \mathrm{~Hz}, 1 \mathrm{H}) ;{ }^{13} \mathrm{C} \mathrm{NMR}\left(126 \mathrm{MHz}, \mathrm{CDCl}_{3}\right) \delta$ 174.6, 158.4, $143.2,134.2,128.4,127.7,126.8,126.5,113.9,55.351 .8,50.4,43.7,41.9,29.3$; IR (thin film) $v$ $3062,3030,2996,2951,2835,1728,1611,1513,1455,1435,1247,1177,1033,821,762,665$ $\mathrm{cm}^{-1}$; HRMS (ESI) $m / \mathrm{z}$ calcd for $\mathrm{C}_{19} \mathrm{H}_{20} \mathrm{O}_{3} \mathrm{Na}(\mathrm{M}+\mathrm{Na})^{+}$319.1305, found 319.1301.

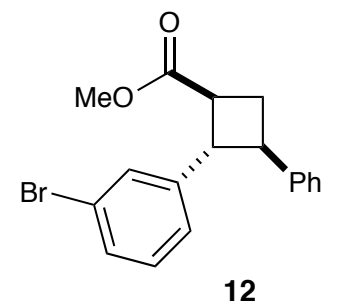

Methyl (1S,2S,3R)-2-(3-bromophenyl)-3-phenylcyclobutane-1-carboxylate (12). The title compound was prepared according to General Procedure C using methyl (E)-3-(3bromophenyl)acrylate $(96.4 \mathrm{mg}, 0.400 \mathrm{mmol}),\left[\operatorname{Ir}(\mathrm{Fppy})_{2}(\mathrm{dtbpy})\left(\mathrm{PF}_{6}\right)\right](3.8 \mathrm{mg}, 0.004 \mathrm{mmol})$, styrene $(0.23 \mathrm{~mL}, 2.00 \mathrm{mmol})$, oxazaborolidine $6 \mathbf{f}(1.0 \mathrm{~mL}, 0.100 \mathrm{mmol})$, and $\mathrm{CH}_{2} \mathrm{Cl}_{2}(12.5 \mathrm{~mL})$. 
The crude material resulted in a 5:1 mixture of diastereomers that was purified by flash chromatography ( $\mathrm{SiO}_{2}$, Column 1: 4\% EtOAc:hexanes, Column 2: 4\% MTBE:pentane). Yield: $113.9 \mathrm{mg}$ of a clear oil $(82 \%, 0.330 \mathrm{mmol}) ; 95 \%$ ee (Daicel CHIRALPAK ${ }^{\circledR}$ OD-H, $5-50 \%$ IPA:hexanes over $13 \mathrm{~min}$, flow rate of $\left.1 \mathrm{~mL} / \mathrm{min} ; \mathrm{t}_{1}=5.39 \mathrm{~min}, \mathrm{t}_{2}=7.77 \mathrm{~min}\right) .[\alpha]_{\mathrm{D}}{ }^{20}=-42.2^{\circ}$ $\left(\mathrm{c}=1.0, \mathrm{CH}_{2} \mathrm{Cl}_{2}\right) ;{ }^{1} \mathrm{H} \mathrm{NMR}\left(\mathrm{CDCl}_{3}, 500 \mathrm{MHz}\right) \delta 7.41(\mathrm{~s}, 1 \mathrm{H}), 7.37-7.30(\mathrm{~m}, 3 \mathrm{H}), 7.28-7.21(\mathrm{~m}$, 3H), 7.18-7.15 (m, 2H), 3.80 (t, $J=9.7 \mathrm{~Hz}, 1 \mathrm{H}), 3.73(\mathrm{~s}, 3 \mathrm{H}), 3.51$ (td, $J=9.8,8.8 \mathrm{~Hz}, 1 \mathrm{H}), 3.17$ $(\mathrm{td}, J=9.5,9.0 \mathrm{~Hz}, 1 \mathrm{H}), 2.64(\mathrm{dt}, J=10.4,8.4 \mathrm{~Hz}, 1 \mathrm{H}), 2.40(\mathrm{q}, J=10.4 \mathrm{~Hz}, 1 \mathrm{H}) ;{ }^{13} \mathrm{C} \mathrm{NMR}$ $\left(126 \mathrm{MHz}, \mathrm{CDCl}_{3}\right) \delta 174.2,144.4,142.6,130.1,129.9,129.7,128.6,126.76,126.75,125.4$ 122.7, 52.0, 50.2, 43.4, 41.4, 29.6; IR (thin film) v 3063, 3028, 2993, 2951, 1731, 2595, 1565, 1495, 1476, 1456, 1435, 1231, 1162, 1073, 1029, 780, 760, 745, $698 \mathrm{~cm}^{-1}$; HRMS (ESI) $\mathrm{m} / \mathrm{z}$ calcd for $\mathrm{C}_{18} \mathrm{H}_{17} \mathrm{O}_{2} \mathrm{BrH}(\mathrm{M}+\mathrm{H})^{+}$345.0485, found 345.0485.

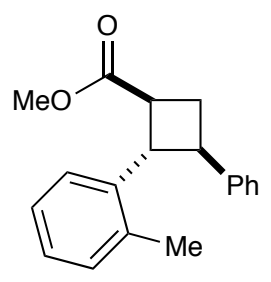

13

Methyl (1S,2S,3R)-3-phenyl-2-(o-tolyl)cyclobutane-1-carboxylate (13). The title compound was prepared according to General Procedure C using methyl (E)-3-(o-tolyl)acrylate (70.5 mg, $0.400 \mathrm{mmol}),\left[\operatorname{Ir}(\mathrm{Fppy})_{2}(\mathrm{dtbpy})\left(\mathrm{PF}_{6}\right)\right](3.8 \mathrm{mg}, 0.004 \mathrm{mmol})$, styrene $(0.23 \mathrm{~mL}, 2.00 \mathrm{mmol})$, oxazaborolidine $\mathbf{6 f}(1.0 \mathrm{~mL}, 0.100 \mathrm{mmol})$, and $\mathrm{CH}_{2} \mathrm{Cl}_{2}(12.5 \mathrm{~mL})$. The crude material resulted in a 9:1 mixture of diastereomers that was purified by flash chromatography $\left(\mathrm{SiO}_{2}\right.$, Column 1: 4\% EtOAc:hexanes, Column 2: 6\% $\mathrm{Et}_{2} \mathrm{O}$ :pentane). Yield: $108.9 \mathrm{mg}$ of a clear oil $(97 \%, 0.388$ mmol); 99\% ee (Daicel CHIRALPAK ${ }^{\circledR}$ OD-H, 5-50\% IPA:hexanes over 13 min, flow rate of 1 $\left.\mathrm{mL} / \mathrm{min} ; \mathrm{t}_{1}=5.14 \mathrm{~min}, \mathrm{t}_{2}=7.30 \mathrm{~min}\right) .[\alpha]_{\mathrm{D}}{ }^{20}=-39.0^{\circ}\left(\mathrm{c}=1.0, \mathrm{CH}_{2} \mathrm{Cl}_{2}\right) ;{ }^{1} \mathrm{H} \mathrm{NMR}\left(\mathrm{CDCl}_{3}, 500\right.$ 
$\mathrm{MHz}) \delta 7.46(\mathrm{~d}, J=7.9 \mathrm{~Hz}, 1 \mathrm{H}), 7.30-7.17(\mathrm{~m}, 6 \mathrm{H}), 7.15-7.08(\mathrm{~m}, 2 \mathrm{H}), 4.03(\mathrm{t}, J=9.8 \mathrm{~Hz}, 1 \mathrm{H})$, $3.68(\mathrm{~s}, 3 \mathrm{H}), 3.59(\mathrm{td}, J=10.0,8.5 \mathrm{~Hz}, 1 \mathrm{H}), 3.19(\mathrm{td}, J=9.7,8.4 \mathrm{~Hz}, 1 \mathrm{H}), 2.62(\mathrm{dt}, J=10.6,8.2$ $\mathrm{Hz}, 1 \mathrm{H}), 2.44(\mathrm{q}, J=10.3 \mathrm{~Hz}, 1 \mathrm{H}), 2.10(\mathrm{~s}, 3 \mathrm{H}) ;{ }^{13} \mathrm{C}$ NMR $\left(126 \mathrm{MHz}, \mathrm{CDCl}_{3}\right) \delta 174.7,143.3$, $139.7,136.3,130.3,128.4,126.7,126.6,126.5,126.2,126.1,51.8,48.1,43.7,41.8,29.1,19.8$; IR (thin film) v 3061, 3026, 2987, 2950, 1730, 1602, 1494, 1456, 1435, 1229, 1200, 1161, 1029, 753, $699 \mathrm{~cm}^{-1}$; HRMS (ESI) $m / \mathrm{z}$ calcd for $\mathrm{C}_{19} \mathrm{H}_{20} \mathrm{O}_{2} \mathrm{NH}_{4}\left(\mathrm{M}+\mathrm{NH}_{4}\right)^{+}$298.1802, found 298.1802.

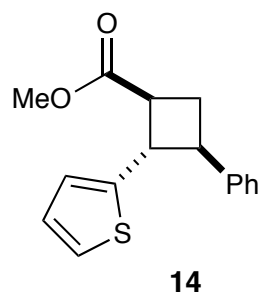

Methyl (1S,2S,3R)-3-phenyl-2-(thiophen-2-yl)cyclobutane-1-carboxylate (14). The title compound was prepared according to General Procedure C using methyl (E)-3-(thiophen-2yl)acrylate $(67.3 \mathrm{mg}, 0.400 \mathrm{mmol}),\left[\operatorname{Ir}(\mathrm{Fppy})_{2}(\mathrm{dtbpy})\left(\mathrm{PF}_{6}\right)\right](3.8 \mathrm{mg}, 0.004 \mathrm{mmol})$, styrene $(0.23$ $\mathrm{mL}, 2.00 \mathrm{mmol})$, oxazaborolidine $6 \mathrm{f}(1.0 \mathrm{~mL}, 0.100 \mathrm{mmol})$, and $\mathrm{CH}_{2} \mathrm{Cl}_{2}(12.5 \mathrm{~mL})$. The crude material resulted in a 4:1 mixture of diastereomers that was purified by flash chromatography $\left(\mathrm{SiO}_{2}\right.$, Column 1: 4\% EtOAc:hexanes, Column 2: 6\% $\mathrm{Et}_{2} \mathrm{O}$ :pentane). Yield: $73.8 \mathrm{mg}$ of a clear oil (68\%, 0.271 mmol); 93\% ee (Daicel CHIRALPAK® OD-H, 5-50\% IPA:hexanes over 13 min, flow rate of $\left.1 \mathrm{~mL} / \mathrm{min} ; \mathrm{t}_{1}=5.88 \mathrm{~min}, \mathrm{t}_{2}=7.36 \mathrm{~min}\right) .[\alpha]_{\mathrm{D}}{ }^{20}=-39.6^{\circ}\left(\mathrm{c}=1.0, \mathrm{CH}_{2} \mathrm{Cl}_{2}\right) ;{ }^{1} \mathrm{H}$ NMR $\left(\mathrm{CDCl}_{3}, 500 \mathrm{MHz}\right) \delta 7.34-7.29(\mathrm{~m}, 2 \mathrm{H}), 7.29-7.25(\mathrm{~m}, 2 \mathrm{H}), 7.25-7.20(\mathrm{~m}, 1 \mathrm{H}), 7.18$ (ap $\mathrm{d}, J=5.1 \mathrm{~Hz}, 1 \mathrm{H}), 6.96-6.93(\mathrm{~m}, 1 \mathrm{H}), 6.91-6.89(\mathrm{~m}, 1 \mathrm{H}), 3.94(\mathrm{t}, J=9.6 \mathrm{~Hz}, 1 \mathrm{H}), 3.72(\mathrm{~s}, 3 \mathrm{H})$, $3.55(\mathrm{dt}, J=9.8,8.7 \mathrm{~Hz}, 1 \mathrm{H}), 3.19(\mathrm{dt}, J=9.4,8.9 \mathrm{~Hz}, 1 \mathrm{H}), 2.62(\mathrm{td}, J=10.6,8.4 \mathrm{~Hz}, 1 \mathrm{H}), 2.41$ $(\mathrm{q}, J=10.4 \mathrm{~Hz}, 1 \mathrm{H}) ;{ }^{13} \mathrm{C} \mathrm{NMR}\left(126 \mathrm{MHz}, \mathrm{CDCl}_{3}\right) \delta 174.0,146.0,142.4,128.5,126.9,126.7$, 
$126.67,123.85,123.80,51.9,46.7,45.4,43.5,28.9$; IR (thin film) $v 3063,3026,2989,2950$, 1729, 1602, 1496, 1456, 1435, 1227, 1199, 1160, 1086, 1029, 848, 760, $696 \mathrm{~cm}^{-1}$; HRMS (ESI) $m / \mathrm{z}$ calcd for $\mathrm{C}_{16} \mathrm{H}_{16} \mathrm{O}_{2} \mathrm{SH}(\mathrm{M}+\mathrm{H})^{+}$273.0944, found 273.0940.

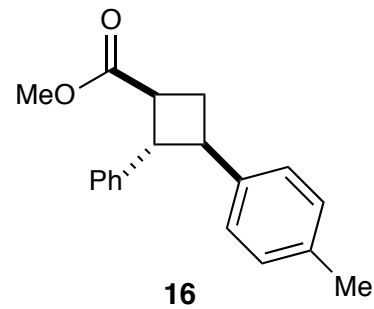

Methyl (1S,2S,3R)-2-phenyl-3-(p-tolyl)cyclobutane-1-carboxylate (16). The title compound was prepared according to General Procedure C using methyl (E)-3-(thiophen-2-yl)acrylate (67.3 $\mathrm{mg}, 0.400 \mathrm{mmol}),\left[\operatorname{Ir}(\mathrm{Fppy})_{2}(\mathrm{dtbpy})\left(\mathrm{PF}_{6}\right)\right](3.8 \mathrm{mg}, 0.004 \mathrm{mmol}), 1$-methyl-4-vinylbenzene $(0.26$ $\mathrm{mL}, 2.00 \mathrm{mmol})$, oxazaborolidine $6 \mathbf{f}(1.0 \mathrm{~mL}, 0.100 \mathrm{mmol})$, and $\mathrm{CH}_{2} \mathrm{Cl}_{2}(12.5 \mathrm{~mL})$. The crude material resulted in a 5:1 mixture of diastereomers that was purified by flash chromatography $\left(\mathrm{SiO}_{2}, 5 \% \mathrm{Et}_{2} \mathrm{O}\right.$ :pentane). Yield: $85.6 \mathrm{mg}$ of a clear oil $(76 \%, 0.305 \mathrm{mmol}) ; 96 \%$ ee (Daicel CHIRALPAK ${ }^{\circledR}$ OD-H, $1-40 \%$ IPA:hexanes over $20 \mathrm{~min}$, flow rate of $1 \mathrm{~mL} / \mathrm{min} ; \mathrm{t}_{1}=6.02 \mathrm{~min}$, $\left.\mathrm{t}_{2}=9.93 \mathrm{~min}\right) \cdot[\alpha]_{\mathrm{D}}{ }^{20}=-31.2^{\circ}\left(\mathrm{c}=0.25, \mathrm{CH}_{2} \mathrm{Cl}_{2}\right) ;{ }^{1} \mathrm{H} \mathrm{NMR}\left(\mathrm{CDCl}_{3}, 500 \mathrm{MHz}\right) \delta 7.31-7.28(\mathrm{~m}$, 2H), 7.25-7.22 (m, 3H), 7.19-7.17 (m, 2H), 7.18 (ap d, $J=7.9 \mathrm{~Hz}, 2 \mathrm{H}), 3.82(\mathrm{t}, J=9.8 \mathrm{~Hz}, 1 \mathrm{H})$, $3.72(\mathrm{~s}, 3 \mathrm{H}), 3.48(\mathrm{td}, J=10.1,8.1 \mathrm{~Hz}, 1 \mathrm{H}), 3.19(\mathrm{td}, J=9.8,8.2 \mathrm{~Hz}, 1 \mathrm{H}), 2.60(\mathrm{dt}, J=10.8,8.2$ $\mathrm{Hz}, 1 \mathrm{H}), 2.38(\mathrm{q}, J=10.4 \mathrm{~Hz}, 1 \mathrm{H}), 2.32(\mathrm{~s}, 3 \mathrm{H}),{ }^{13} \mathrm{C} \mathrm{NMR}\left(126 \mathrm{MHz}, \mathrm{CDCl}_{3}\right) \delta 174.66,142.18$, $140.14,136.16,129.14,128.44,126.78,126.64,126.58,51.84,50.86,43.28,41.35,29.76$, 21.05; IR (thin film) v 3024, 2948, 1728, 1602, 1510, 1201, 1162, 908, 729, $697 \mathrm{~cm}^{-1}$; HRMS (ESI) $m / z$ calcd for $\mathrm{C}_{19} \mathrm{H}_{20} \mathrm{O}_{2} \mathrm{Na}(\mathrm{M}+\mathrm{Na})^{+} 303.1356$, found 303.1353 . 


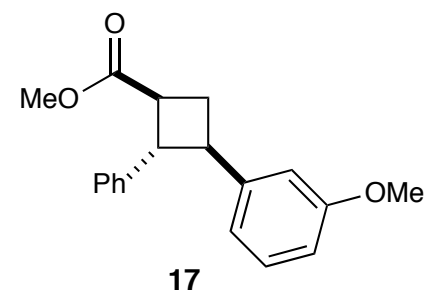

Methyl (1S,2S,3R)-3-(3-methoxyphenyl)-2-phenylcyclobutane-1-carboxylate (17). The title compound was prepared according to General Procedure C using methyl cinnamate (64.9 mg, $0.400 \mathrm{mmol}),\left[\operatorname{Ir}(\mathrm{Fppy})_{2}(\mathrm{dtbpy})\left(\mathrm{PF}_{6}\right)\right](3.8 \mathrm{mg}, 0.004 \mathrm{mmol}), 3$-vinylanisole $(0.28 \mathrm{~mL}, 2.00$ mmol), oxazaborolidine $6 \mathbf{f}(1.0 \mathrm{~mL}, 0.100 \mathrm{mmol})$, and $\mathrm{CH}_{2} \mathrm{Cl}_{2}(12.5 \mathrm{~mL})$. The crude material resulted in a 6:1 mixture of diastereomers that was purified by flash chromatography $\left(\mathrm{SiO}_{2}, 6 \%\right.$ EtOAc:hexanes). Yield: $78.9 \mathrm{mg}$ of a clear oil (67\%, $0.266 \mathrm{mmol}) ; 90 \%$ ee (Daicel CHIRALPAK® OD-H, 5-50\% IPA:hexanes over $13 \mathrm{~min}$, flow rate of $1 \mathrm{~mL} / \mathrm{min} ; \mathrm{t}_{1}=6.10 \mathrm{~min}$, $\left.\mathrm{t}_{2}=6.90 \mathrm{~min}\right) .[\alpha]_{\mathrm{D}}{ }^{20}=-34.0^{\circ}\left(\mathrm{c}=1.0, \mathrm{CH}_{2} \mathrm{Cl}_{2}\right) ;{ }^{1} \mathrm{H} \mathrm{NMR}\left(\mathrm{CDCl}_{3}, 500 \mathrm{MHz}\right) \delta 7.34-7.27(\mathrm{~m}$, 2H), 7.26-7.18 (m, 3H), $6.87(\mathrm{~d}, J=7.6 \mathrm{~Hz}, 1 \mathrm{H}), 6.81$ (br t, $J=1.9 \mathrm{~Hz}, 1 \mathrm{H}), 6.76(\mathrm{dd}, J=8.2$, $2.3 \mathrm{~Hz}, 1 \mathrm{H}), 3.84(\mathrm{t}, J=9.8 \mathrm{~Hz}, 1 \mathrm{H}), 3.79(\mathrm{~s}, 3 \mathrm{H}), 3.72(\mathrm{~s}, 3 \mathrm{H}), 3.50(\mathrm{td}, J=10.1,8.2 \mathrm{~Hz}, 1 \mathrm{H})$, $3.19(\mathrm{td}, J=9.8,8.2 \mathrm{~Hz}, 1 \mathrm{H}), 2.62(\mathrm{dt}, J=10.7,8.2 \mathrm{~Hz}, 1 \mathrm{H}), 2.40(\mathrm{q}, J=10.4 \mathrm{~Hz}, 1 \mathrm{H}) ;{ }^{13} \mathrm{C}$ NMR $\left(126 \mathrm{MHz}, \mathrm{CDCl}_{3}\right) \delta 174.5,159.7,144.9,142.0,129.5,128.5,126.7,126.6,119.2,112.7$, 111.7, 55.2, 51.9, 50.7, 43.5, 41.4, 29.6; IR (thin film) v 3058, 3028, 2995, 2950, 2835, 1728, 1601, 1583, 1489, 1452, 1435, 1289, 1260, 1230, 1198, 1158, 1039, 774, 755, $696 \mathrm{~cm}^{-1}$; HRMS (ESI) $m / \mathrm{z}$ calcd for $\mathrm{C}_{19} \mathrm{H}_{20} \mathrm{O}_{3} \mathrm{H}(\mathrm{M}+\mathrm{H})^{+} 297.1485$, found 297.1483.

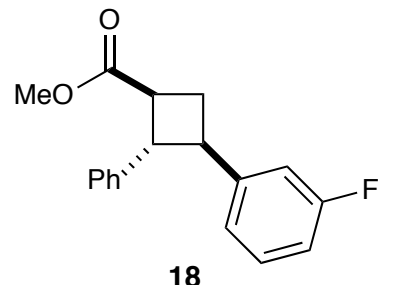


Methyl (1S,2S,3R)-3-(3-fluorophenyl)-2-phenylcyclobutane-1-carboxylate (18). The title compound was prepared according to General Procedure C using methyl cinnamate (64.9 mg, $0.400 \mathrm{mmol}),\left[\operatorname{Ir}(\mathrm{Fppy})_{2}(\mathrm{dtbpy})\left(\mathrm{PF}_{6}\right)\right](3.8 \mathrm{mg}, 0.004 \mathrm{mmol}), 1$-fluoro-3-vinylbezne $(0.24 \mathrm{~mL}$, $2.00 \mathrm{mmol})$, oxazaborolidine $6 \mathbf{f}(1.0 \mathrm{~mL}, 0.100 \mathrm{mmol})$, and $\mathrm{CH}_{2} \mathrm{Cl}_{2}(12.5 \mathrm{~mL})$. The crude material resulted in a 6:1 mixture of diastereomers that was purified by flash chromatography $\left(\mathrm{SiO}_{2}, 5 \% \mathrm{Et}_{2} \mathrm{O}\right.$ : Pentane. Yield: $104.6 \mathrm{mg}$ of a clear oil $(92 \%, 0.368 \mathrm{mmol}) ; 97 \%$ ee (Daicel CHIRALPAK® IC, $1-40 \%$ IPA:hexanes over $20 \mathrm{~min}$, flow rate of $1 \mathrm{~mL} / \mathrm{min} ; \mathrm{t}_{1}=6.35 \mathrm{~min}, \mathrm{t}_{2}=$ $6.83 \mathrm{~min}) .[\alpha]_{\mathrm{D}}{ }^{20}=-41.9^{\circ}\left(\mathrm{c}=1.14, \mathrm{CH}_{2} \mathrm{Cl}_{2}\right) ;{ }^{1} \mathrm{H} \mathrm{NMR}\left(\mathrm{CDCl}_{3}, 500 \mathrm{MHz}\right) \delta 7.33-7.30(\mathrm{~m}$, 2H), 7.27-7.21 (m, 4H), $7.03(\mathrm{~d}, J=7.6 \mathrm{~Hz}, 1 \mathrm{H}), 6.97(\mathrm{dt}, J=10.0,2.2 \mathrm{~Hz}, 1 \mathrm{H}), 6.90(\mathrm{td}, J=$ 8.1, 2.6 Hz, 1H), $3.81(\mathrm{t}, J=9.7 \mathrm{~Hz}, 1 \mathrm{H}), 3.72(\mathrm{~s}, 3 \mathrm{H}), 3.53(\mathrm{td}, J=10.1,8.1 \mathrm{~Hz}, 1 \mathrm{H}), 3.21(\mathrm{td}, J$ $=9.8,8.2 \mathrm{~Hz}, 1 \mathrm{H}), 2.63(\mathrm{dt}, J=10.9,8.2 \mathrm{~Hz}, 1 \mathrm{H}), 2.38(\mathrm{q}, J=10.3 \mathrm{~Hz}, 1 \mathrm{H}) ;{ }^{13} \mathrm{C}$ NMR $(126$ $\left.\mathrm{MHz}, \mathrm{CDCl}_{3}\right) \delta 174.38,162.99(\mathrm{~d}, J=245.9 \mathrm{~Hz}), 162.01,145.82(\mathrm{~d}, J=7.0 \mathrm{~Hz}), 141.67,129.95$ $(\mathrm{d}, J=8.3 \mathrm{~Hz}), 128.58,126.89,126.58,122.46(\mathrm{~d}, J=2.8 \mathrm{~Hz}), 113.81,113.64,113.56,113.39$, 51.92, 50.79, 43.13, 41.41, 29.41; ${ }^{19} \mathrm{~F}$ NMR (376 $\left.\mathrm{MHz}, \mathrm{CDCl}_{3}\right) \delta-113.2(\mathrm{~s}, \mathrm{~F})$; IR (thin film) $v$ 3030, 2991, 2952, 1727, 1587, 1442, 1230, 1202, 1156, 907, 728, $694 \mathrm{~cm}^{-1}$; HRMS (ESI) $\mathrm{m} / \mathrm{z}$ calcd for $\mathrm{C}_{18} \mathrm{H}_{17} \mathrm{FO}_{2} \mathrm{Na}(\mathrm{M}+\mathrm{Na})^{+}$307.1105, found 307.1101.

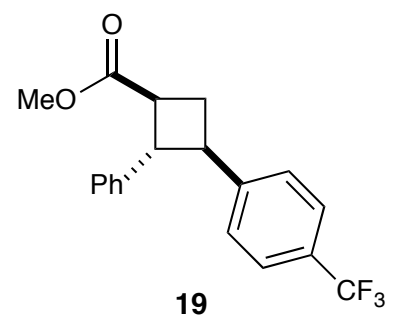

Methyl (1S,2S,3R)-2-phenyl-3-(4-(trifluoromethyl)phenyl)cyclobutane-1-carboxylate (19).

The title compound was prepared according to General Procedure $\mathrm{C}$ using methyl cinnamate $(64.9 \quad \mathrm{mg}, \quad 0.400 \quad \mathrm{mmol}), \quad\left[\operatorname{Ir}(\mathrm{Fppy})_{2}(\mathrm{dtbpy})\left(\mathrm{PF}_{6}\right)\right] \quad\left(\begin{array}{lllll}3.8 & \mathrm{mg}, & 0.004 & \mathrm{mmol}\end{array}\right), \quad 4-$ 
(trifluoromethyl)styrene $(0.30 \mathrm{~mL}, 2.00 \mathrm{mmol})$, oxazaborolidine $6 \mathbf{f}(1.0 \mathrm{~mL}, 0.100 \mathrm{mmol})$, and $\mathrm{CH}_{2} \mathrm{Cl}_{2}(12.5 \mathrm{~mL})$. The crude material resulted in a $8: 1$ mixture of diastereomers that was purified by flash chromatography $\left(\mathrm{SiO}_{2}, 5 \%\right.$ EtOAc:hexanes). Yield: $100.5 \mathrm{mg}$ of a clear oil (75\%, $0.301 \mathrm{mmol}) ; 95 \%$ ee (Daicel CHIRALPAK ${ }^{\circledR}$ OD-H, 5-50\% IPA:hexanes over $13 \mathrm{~min}$, flow rate of $\left.1 \mathrm{~mL} / \mathrm{min} ; \mathrm{t}_{1}=5.21 \mathrm{~min}, \mathrm{t}_{2}=5.94 \mathrm{~min}\right) .[\alpha]_{\mathrm{D}}{ }^{20}=-42.8^{\circ}\left(\mathrm{c}=1.0, \mathrm{CH}_{2} \mathrm{Cl}_{2}\right) ;{ }^{1} \mathrm{H} \mathrm{NMR}$ $\left(\mathrm{CDCl}_{3}, 500 \mathrm{MHz}\right) \delta 7.56(\mathrm{~d}, J=8.0 \mathrm{~Hz}, 2 \mathrm{H}), 7.37(\mathrm{~d}, J=8.0 \mathrm{~Hz}, 2 \mathrm{H}), 7.34-7.29(\mathrm{~m}, 2 \mathrm{H}), 7.27-$ $7.22(\mathrm{~m}, 4 \mathrm{H}), 3.84(\mathrm{t}, J=9.7 \mathrm{~Hz}, 1 \mathrm{H}), 3.73(\mathrm{~s}, 3 \mathrm{H}), 3.59(\mathrm{td}, J=10.1,8.3 \mathrm{~Hz}, 1 \mathrm{H}), 3.24(\mathrm{td}, J=$ 9.8, 8.2 Hz, 1H), $2.66(\mathrm{dt}, J=10.8,8.2 \mathrm{~Hz}, 1 \mathrm{H}), 2.42(\mathrm{q}, J=10.4 \mathrm{~Hz}, 1 \mathrm{H}) ;{ }^{13} \mathrm{C}$ NMR $(126 \mathrm{MHz}$, $\left.\mathrm{CDCl}_{3}\right) \delta 174.3,147.1($ br d, $J=1.2 \mathrm{~Hz}), 141.5,128.9(\mathrm{q}, J=32.5 \mathrm{~Hz}), 128.6,127.1,127.0$, 126.6, $125.4(\mathrm{q}, J=3.8 \mathrm{~Hz}), 124.2(\mathrm{q}, J=271.6 \mathrm{~Hz}), 52.0,50.8,43.2,41.5,29.3 ;{ }^{19} \mathrm{~F}$ NMR $(376$ $\left.\mathrm{MHz}, \mathrm{CDCl}_{3}\right) \delta-62.4$ (s, 3F); IR (thin film) v 3029, 2991, 2954, 1731, 1619, 1497, 1448, 1437, 1421, 1324, 1232, 1201, 1162, 1113, 1068, 1017, 842, 822, 757, 747, $699 \mathrm{~cm}^{-1}$; HRMS (ESI) $\mathrm{m} /$ $\mathrm{z}$ calcd for $\mathrm{C}_{19} \mathrm{H}_{17} \mathrm{O}_{2} \mathrm{~F}_{3} \mathrm{NH}_{4}\left(\mathrm{M}+\mathrm{NH}_{4}\right)^{+} 352.1519$, found 352.1518 .

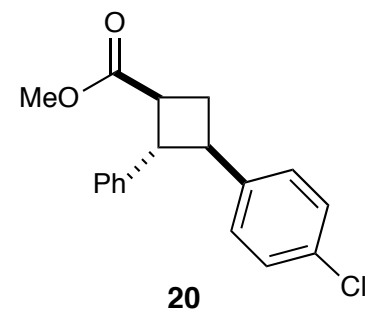

Methyl (1S,2S,3R)-3-(4-chlorophenyl)-2-phenylcyclobutane-1-carboxylate (20). The title compound was prepared according to General Procedure C using methyl cinnamate (64.9 mg, $0.400 \mathrm{mmol}),\left[\operatorname{Ir}(\mathrm{Fppy})_{2}(\mathrm{dtbpy})\left(\mathrm{PF}_{6}\right)\right](3.8 \mathrm{mg}, 0.004 \mathrm{mmol})$, 1-chloro-4-vinylbenzne $(0.24 \mathrm{~mL}$, $2.00 \mathrm{mmol})$, oxazaborolidine $6 \mathrm{f}(1.0 \mathrm{~mL}, 0.100 \mathrm{mmol})$, and $\mathrm{CH}_{2} \mathrm{Cl}_{2}(12.5 \mathrm{~mL})$. The crude material resulted in a 5:1 mixture of diastereomers that was purified by flash chromatography $\left(\mathrm{SiO}_{2}, 3 \% \mathrm{Et}_{2} \mathrm{O}\right.$ : Pentane). Yield: $102.9 \mathrm{mg}$ of a clear oil (85\%, $\left.0.342 \mathrm{mmol}\right) ; 96 \%$ ee (Daicel 
CHIRALPAK® OD-H, 1-40\% IPA:hexanes over $20 \mathrm{~min}$, flow rate of $1 \mathrm{~mL} / \mathrm{min} ; \mathrm{t}_{1}=6.59 \mathrm{~min}$, $\left.\mathrm{t}_{2}=7.13 \mathrm{~min}\right) .[\alpha]_{\mathrm{D}}{ }^{20}=-61.9^{\circ}\left(\mathrm{c}=1.23, \mathrm{CH}_{2} \mathrm{Cl}_{2}\right) ;{ }^{1} \mathrm{H} \mathrm{NMR}\left(\mathrm{CDCl}_{3}, 500 \mathrm{MHz}\right) \delta 7.33-7.29(\mathrm{~m}$, 2H), 7.28-7.25 (m, 2H), 7.25-7.21 (m, 3H), $7.20(\mathrm{ap} \mathrm{d}, J=8.5 \mathrm{~Hz}, 2 \mathrm{H}), 3.78(\mathrm{t}, J=9.8 \mathrm{~Hz}, 1 \mathrm{H})$, $3.72(\mathrm{~s}, 3 \mathrm{H}), 3.49(\mathrm{td}, J=10.1,8.2 \mathrm{~Hz}, 1 \mathrm{H}), 3.21(\mathrm{td}, J=9.8,8.2 \mathrm{~Hz}, 1 \mathrm{H}), 2.62(\mathrm{dt}, J=10.8,8.2$ $\mathrm{Hz}, 1 \mathrm{H}), 2.37$ (q, $J=10.4 \mathrm{~Hz}, 1 \mathrm{H}) ;{ }^{13} \mathrm{C} \mathrm{NMR}\left(126 \mathrm{MHz}, \mathrm{CDCl}_{3}\right) \delta 174.44,141.69,141.60$, $132.31,128.59,128.57,128.21,126.87,126.55,51.91,50.98,43.01,41.32,29.48$; IR (thin film) v 3029, 2991, 2951, 1727, 1601, 1492, 1226, 1203, 906, 728, $649 \mathrm{~cm}^{-1}$; HRMS (ESI) $\mathrm{m} / \mathrm{z}$ calcd for $\mathrm{C}_{18} \mathrm{H}_{17} \mathrm{ClO}_{2} \mathrm{Na}(\mathrm{M}+\mathrm{Na})^{+} 323.0809$, found 323.0806.

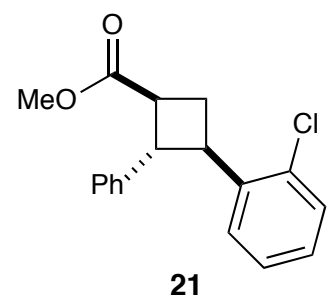

Methyl (1S,2S,3R)-3-(2-chlorophenyl)-2-phenylcyclobutane-1-carboxylate (21). The title compound was prepared according to General Procedure C using methyl cinnamate (64.9 mg, $0.400 \mathrm{mmol}),\left[\operatorname{Ir}(\mathrm{Fppy})_{2}(\mathrm{dtbpy})\left(\mathrm{PF}_{6}\right)\right](3.8 \mathrm{mg}, 0.004 \mathrm{mmol})$, 2-chlorostyrene $(0.26 \mathrm{~mL}, 2.00$ mmol), oxazaborolidine $6 f(1.0 \mathrm{~mL}, 0.100 \mathrm{mmol})$, and $\mathrm{CH}_{2} \mathrm{Cl}_{2}(12.5 \mathrm{~mL})$. The crude material resulted in a 9:1 mixture of diastereomers that was purified by flash chromatography $\left(\mathrm{SiO}_{2}\right.$, Column 1: 4\% EtOAc:hexanes, Column 2: 0.5\% EtOAc:PhMe). Yield: $93.5 \mathrm{mg}$ of a clear oil (78\%, $0.311 \mathrm{mmol}) ; 96 \%$ ee (Daicel CHIRALPAK® OD-H, 5-50\% IPA:hexanes over 13 min, flow rate of $\left.1 \mathrm{~mL} / \mathrm{min} ; \mathrm{t}_{1}=5.08 \mathrm{~min}, \mathrm{t}_{2}=7.37 \mathrm{~min}\right) .[\alpha]_{\mathrm{D}}{ }^{20}=-10.8^{\circ}\left(\mathrm{c}=1.0, \mathrm{CH}_{2} \mathrm{Cl}_{2}\right) ;{ }^{1} \mathrm{H} \mathrm{NMR}$ $\left(\mathrm{CDCl}_{3}, 500 \mathrm{MHz}\right) \delta 7.45(\mathrm{dd}, J=7.8,1.5 \mathrm{~Hz}, 1 \mathrm{H}), 7.35-7.20(\mathrm{~m}, 7 \mathrm{H}), 7.16(\mathrm{td}, J=7.7,1.7 \mathrm{~Hz}$, 1H), $4.05(\mathrm{t}, J=9.8 \mathrm{~Hz}, 1 \mathrm{H}), 3.95(\mathrm{td}, J=10.2,8.1 \mathrm{~Hz}, 1 \mathrm{H}), 3.73(\mathrm{~s}, 3 \mathrm{H}), 3.20(\mathrm{td}, J=9.6,8.3$ $\mathrm{Hz}, 1 \mathrm{H}), 2.83(\mathrm{dt}, J=10.7,8.3 \mathrm{~Hz}, 1 \mathrm{H}), 2.20(\mathrm{q}, J=10.2 \mathrm{~Hz}, 1 \mathrm{H}) ;{ }^{13} \mathrm{C} \mathrm{NMR}\left(126 \mathrm{MHz}, \mathrm{CDCl}_{3}\right)$ 
$\delta 174.6,141.7,140.2,133.6,129.4,128.5,127.8,127.7,127.0,126.8,126.6,51.9,48.1,42.1$, 40.0, 30.4; IR (thin film) v 3063, 3027, 2995, 2951, 1730, 1497, 1476, 1436, 1230, 1199, 1164 , $1034,752,698 \mathrm{~cm}^{-1}$; HRMS (ESI) $\mathrm{m} / \mathrm{z}$ calcd for $\mathrm{C}_{18} \mathrm{H}_{17} \mathrm{ClO}_{2} \mathrm{NH}_{4}\left(\mathrm{M}+\mathrm{NH}_{4}\right)^{+} 318.1255$, found 318.1253.

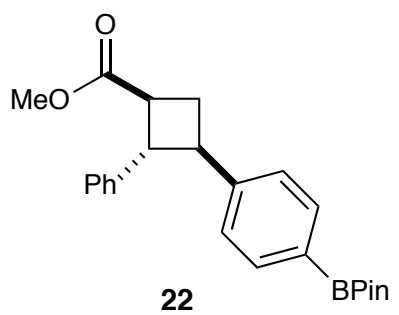

Methyl (1S,2S,3R)-2-phenyl-3-(4-(4,4,5,5-tetramethyl-1,3,2-dioxaborolan-2-yl)phenyl)cyclobutane-1-carboxylate (22). The title compound was prepared according to General Procedure C using methyl cinnamate $(64.9 \mathrm{mg}, 0.400 \mathrm{mmol})$, [Ir(Fppy $\left.)_{2}(\mathrm{dtbpy})\left(\mathrm{PF}_{6}\right)\right](3.8 \mathrm{mg}, 0.004 \mathrm{mmol})$, 4-vinylphenylboronic acid $(460.2 \mathrm{mg}, 2.00 \mathrm{mmol})$, oxazaborolidine $6 f(1.0 \mathrm{~mL}, 0.100 \mathrm{mmol})$, and $\mathrm{CH}_{2} \mathrm{Cl}_{2}(12.5 \mathrm{~mL})$, an irradiation time of $76 \mathrm{~h}$. The crude material resulted in a 6:1 mixture of diastereomers that was purified by flash chromatography $\left(\mathrm{SiO}_{2}, 5 \% \mathrm{Et}_{2} \mathrm{O}\right.$ : Pentane). Yield: $120.7 \mathrm{mg}$ of a clear oil $(77 \%, 0.307 \mathrm{mmol}) ; 92 \%$ ee (Daicel CHIRALPAK® IC, $1-40 \%$ IPA:hexanes over $20 \mathrm{~min}$, flow rate of $\left.1 \mathrm{~mL} / \mathrm{min} ; \mathrm{t}_{1}=5.91 \mathrm{~min}, \mathrm{t}_{2}=6.35 \mathrm{~min}\right) .[\alpha]_{\mathrm{D}}^{20}=-46.2^{\circ}$ $\left(\mathrm{c}=0.81, \mathrm{CH}_{2} \mathrm{Cl}_{2}\right) ;{ }^{1} \mathrm{H} \mathrm{NMR}\left(\mathrm{CDCl}_{3}, 500 \mathrm{MHz}\right) \delta 7.77-7.75(\mathrm{~m}, 2 \mathrm{H}), 7.30-7.27(\mathrm{~m}, 4 \mathrm{H}), 7.23-$ $7.20(\mathrm{~m}, 3 \mathrm{H}), 3.85(\mathrm{t}, J=9.8 \mathrm{~Hz}, 1 \mathrm{H}), 3.72(\mathrm{~s}, 3 \mathrm{H}), 3.52(\mathrm{td}, J=10.1,8.1 \mathrm{~Hz}, 1 \mathrm{H}), 3.22(\mathrm{td}, J=$ 9.9, 8.2 Hz, 1H), $2.62(\mathrm{dt}, J=11.0,8.3 \mathrm{~Hz}, 1 \mathrm{H}), 2.42(\mathrm{q}, J=10.4 \mathrm{~Hz}, 1 \mathrm{H}), 1.33(\mathrm{~s}, 12 \mathrm{H}) ;{ }^{13} \mathrm{C}$ NMR $\left(126 \mathrm{MHz}, \mathrm{CDCl}_{3}\right) \delta 174.55,146.30,142.00,135.02,128.47,126.70,126.54,126.33$, 83.72, 51.87, 50.68, 43.87, 41.28, 29.50, 24.85; ${ }^{11} \mathrm{~B}$ NMR (160 MHz, CDCl3): $\delta 30.5$ (b, B); IR $\left(\right.$ thin film) $\vee 2380,1730,1608,1492,1441,1396,1357,1322,1267,1144,1088,736,699 \mathrm{~cm}^{-1}$; HRMS (ESI) $\mathrm{m} / \mathrm{z}$ calcd for $\mathrm{C}_{24} \mathrm{H}_{29} \mathrm{BO}_{4} \mathrm{Na}(\mathrm{M}+\mathrm{Na})^{+} 415.2051$, found 415.2048 . 


\section{General Procedure D: Racemic [2+2] Photocycloaddition of Cinnamic Esters}

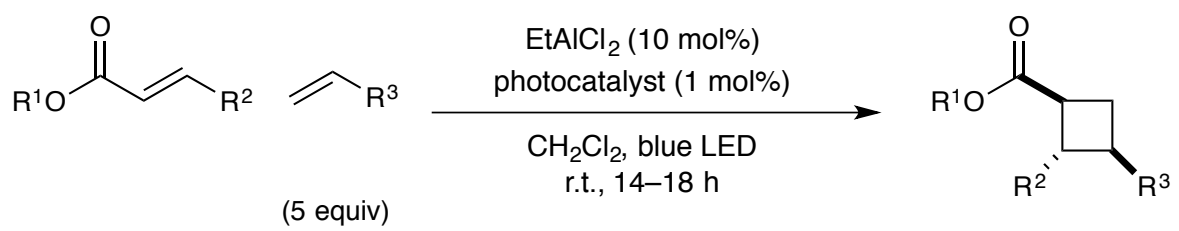

To a solution of cinnamic ester (1 equiv) and photocatalyst $(1 \mathrm{~mol} \%)$ in $\mathrm{CH}_{2} \mathrm{Cl}_{2}(0.03 \mathrm{M})$ was added $\mathrm{EtAlCl}_{2}(10 \mathrm{~mol} \%, 1 \mathrm{M}$ in hexanes) dropwise via syringe. After five minutes, styrene (5 equiv) was added dropwise via syringe. The reaction mixture was irradiated with a $15 \mathrm{~W}$ blue LED lamp at a distance of $15 \mathrm{~cm}$ for $14-48 \mathrm{~h}$. The reaction was quenched with $1 \mathrm{M} \mathrm{HCl}(10$ $\mathrm{mL})$, and the aqueous layer was extracted with $\mathrm{CH}_{2} \mathrm{Cl}_{2}(3 \times 10 \mathrm{~mL})$. The combined organic extracts were washed with saturated $\mathrm{NaHCO}_{3}$ solution $(1 \times 20 \mathrm{~mL})$, washed with brine $(1 \times 20$ $\mathrm{mL}$ ), dried over $\mathrm{MgSO}_{4}$, filtered, and concentrated in vacuo. The crude residue was purified using flash chromatography, yielding the desired [2+2] cycloadduct.

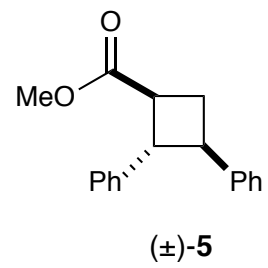

Methyl $\left(1 S^{*}, 2 S^{*}, 3 R^{*}\right)-2,3-$ diphenylcyclobutane-1-carboxylate (5). The title compound was prepared according to General Procedure D using methyl cinnamate (64.9 mg, $0.400 \mathrm{mmol}$ ), $\left[\operatorname{Ir}(\mathrm{ppy})_{2}(\mathrm{dtbpy})\left(\mathrm{PF}_{6}\right)\right](3.7 \mathrm{mg}, 0.004 \mathrm{mmol})$, styrene $(0.23 \mathrm{~mL}, 2.00 \mathrm{mmol}), \mathrm{EtAlCl}_{2}(40 \mu \mathrm{L}$, $0.040 \mathrm{mmol}), \mathrm{CH}_{2} \mathrm{Cl}_{2}(13.5 \mathrm{~mL})$, and an irradiation time of $14 \mathrm{~h}$. The crude material resulted in a 2:1 mixture of diastereomers that was purified by flash chromatography $\left(\mathrm{SiO}_{2}, 5 \%\right.$ $\mathrm{Et}_{2} \mathrm{O}$ :pentane). Combined yield: $94.7 \mathrm{mg}$ of a clear oil $(89 \%, 0.356 \mathrm{mmol})$. All spectroscopic data match those reported above. 


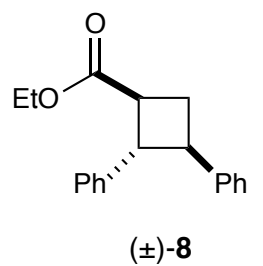

Ethyl $\left(1 S^{*}, 2 S^{*}, 3 R^{*}\right)-2,3-$ diphenylcyclobutane-1-carboxylate $(8)$. The title compound was prepared according to General Procedure D using ethyl cinnamate (70.5 mg, $0.400 \mathrm{mmol}$ ), $\left[\operatorname{Ir}(\text { ppy })_{2}(\mathrm{dtbpy})\left(\mathrm{PF}_{6}\right)\right](3.7 \mathrm{mg}, 0.004 \mathrm{mmol})$, styrene $(0.23 \mathrm{~mL}, 2.00 \mathrm{mmol}), \mathrm{EtAlCl}_{2}(40 \mu \mathrm{L}$, $0.040 \mathrm{mmol}), \mathrm{CH}_{2} \mathrm{Cl}_{2}(13.5 \mathrm{~mL})$, and an irradiation time of $14 \mathrm{~h}$. The crude material resulted in a 2:1 mixture of diastereomers that was purified by flash chromatography $\left(\mathrm{SiO}_{2}, 4 \%\right.$ EtOAc:hexanes). Combined yield: $102.3 \mathrm{mg}$ of a clear oil (91\%, $0.365 \mathrm{mmol})$. All spectroscopic data match those reported above.

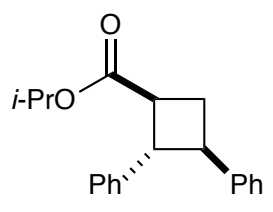

(土)-9

Isopropyl $\left(1 S^{*}, 2 S^{*}, 3 R^{*}\right)-2,3-$ diphenylcyclobutane-1-carboxylate (9). The title compound was prepared according to General Procedure D using isopropyl cinnamate $(76.1 \mathrm{mg}, 0.400 \mathrm{mmol})$, $\left[\operatorname{Ir}(\mathrm{Fppy})_{2}(\mathrm{dtbpy})\left(\mathrm{PF}_{6}\right)\right](3.8 \mathrm{mg}, 0.004 \mathrm{mmol})$, styrene $(0.23 \mathrm{~mL}, 2.00 \mathrm{mmol}), \mathrm{EtAlCl}_{2}(40 \mu \mathrm{L}$, $0.040 \mathrm{mmol}), \mathrm{CH}_{2} \mathrm{Cl}_{2}(13.5 \mathrm{~mL})$, and an irradiation time of $14 \mathrm{~h}$. The crude material resulted in a 2.2:1 mixture of diastereomers that was purified by flash chromatography $\left(\mathrm{SiO}_{2}, 4 \%\right.$ EtOAc:hexanes). Combined yield: $115.2 \mathrm{mg}$ of a clear oil $(98 \%, 0.391 \mathrm{mmol})$. All spectroscopic data match those reported above. 


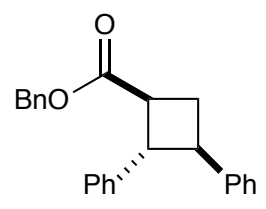

$( \pm)-10$

Benzyl $\left(1 S^{*}, 2 S^{*}, 3 R^{*}\right)-2,3-$ diphenylcyclobutane-1-carboxylate (10). The title compound was prepared according to General Procedure D using benzyl cinnamate (95.3 mg, $0.400 \mathrm{mmol}$ ), $\left[\operatorname{Ir}(\text { Fppy })_{2}(\mathrm{dtbpy})\left(\mathrm{PF}_{6}\right)\right](3.8 \mathrm{mg}, 0.004 \mathrm{mmol})$, styrene $(0.23 \mathrm{~mL}, 2.00 \mathrm{mmol}), \mathrm{EtAlCl}_{2}(40 \mu \mathrm{L}$, $0.040 \mathrm{mmol}), \mathrm{CH}_{2} \mathrm{Cl}_{2}(13.5 \mathrm{~mL})$, and an irradiation time of $18 \mathrm{~h}$. The crude material resulted in a 2.3:1 mixture of diastereomers that was purified by flash chromatography $\left(\mathrm{SiO}_{2}, 4 \%\right.$ EtOAc:hexanes). Combined yield: $120.0 \mathrm{mg}$ of a clear oil (88\%, $0.350 \mathrm{mmol})$. All spectroscopic data match those reported above.

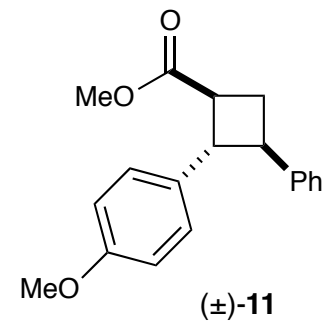

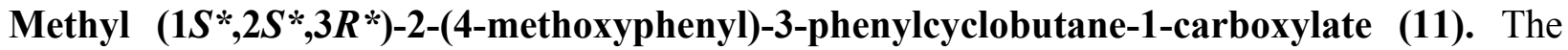
title compound was prepared according to General Procedure D using methyl (E)-3-(4methoxyphenyl)acrylate $(76.9 \mathrm{mg}, 0.400 \mathrm{mmol}),\left[\operatorname{Ir}(\mathrm{Fppy})_{2}(\mathrm{dtbpy})\left(\mathrm{PF}_{6}\right)\right](3.8 \mathrm{mg}, 0.004 \mathrm{mmol})$, styrene $(0.23 \mathrm{~mL}, 2.00 \mathrm{mmol}), \mathrm{EtAlCl}_{2}(40 \mu \mathrm{L}, 0.040 \mathrm{mmol}), \mathrm{CH}_{2} \mathrm{Cl}_{2}(13.5 \mathrm{~mL})$, and an irradiation time of $16 \mathrm{~h}$. The crude material resulted in a 5:1 mixture of diastereomers that was purified by flash chromatography $\left(\mathrm{SiO}_{2}, 8 \%\right.$ EtOAc:hexanes). Combined yield: $108.5 \mathrm{mg}$ of a clear oil $(92 \%, 0.366 \mathrm{mmol})$. All spectroscopic data match those reported above. 


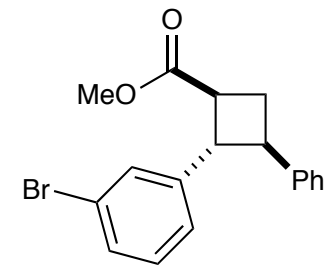

$( \pm)-12$

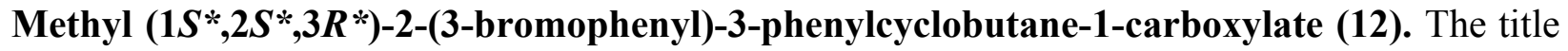
compound was prepared according to General Procedure D using methyl (E)-3-(3bromophenyl)acrylate $(96.4 \mathrm{mg}, 0.400 \mathrm{mmol}),\left[\operatorname{Ir}(\mathrm{Fppy})_{2}(\mathrm{dtbpy})\left(\mathrm{PF}_{6}\right)\right](3.8 \mathrm{mg}, 0.004 \mathrm{mmol})$, styrene $(0.23 \mathrm{~mL}, 2.00 \mathrm{mmol}), \mathrm{EtAlCl}_{2}(40 \mu \mathrm{L}, 0.040 \mathrm{mmol}), \mathrm{CH}_{2} \mathrm{Cl}_{2}(13.5 \mathrm{~mL})$, and an irradiation time of $16 \mathrm{~h}$. The crude material resulted in a $2: 1$ mixture of diastereomers that was purified by flash chromatography $\left(\mathrm{SiO}_{2}, 4 \%\right.$ EtOAc:hexanes). Combined yield: $119.5 \mathrm{mg}$ of a clear oil $(87 \%, 0.346 \mathrm{mmol})$. All spectroscopic data match those reported above.

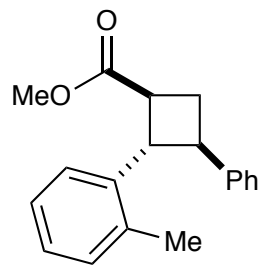

$( \pm)-13$

Methyl $\left(1 S^{*}, 2 S^{*}, 3 R^{*}\right)-3-$ phenyl-2-(o-tolyl)cyclobutane-1-carboxylate $\quad(13)$. The title compound was prepared according to General Procedure D using methyl $(E)$-3-(o-tolyl)acrylate (70.5 mg, $0.400 \mathrm{mmol}),\left[\operatorname{Ir}(\mathrm{Fppy})_{2}(\mathrm{dtbpy})\left(\mathrm{PF}_{6}\right)\right](3.8 \mathrm{mg}, 0.004 \mathrm{mmol})$, styrene $(0.23 \mathrm{~mL}, 2.00$ mmol), EtAlCl $2(40 \mu \mathrm{L}, 0.040 \mathrm{mmol}), \mathrm{CH}_{2} \mathrm{Cl}_{2}(13.5 \mathrm{~mL})$, and an irradiation time of $16 \mathrm{~h}$. The crude material resulted in a 2.5:1 mixture of diastereomers that was purified by flash chromatography $\left(\mathrm{SiO}_{2}, 4 \%\right.$ EtOAc:hexanes). Combined yield: $104.0 \mathrm{mg}$ of a clear oil $(93 \%$, $0.371 \mathrm{mmol})$. All spectroscopic data match those reported above. 


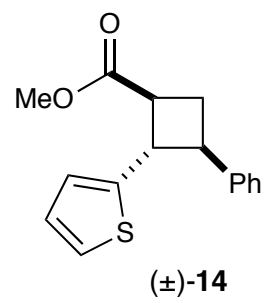

Methyl $\left(1 S^{*}, 2 S^{*}, 3 R^{*}\right)-3$-phenyl-2-(thiophen-2-yl)cyclobutane-1-carboxylate (14). The title compound was prepared according to General Procedure D using methyl (E)-3-(thiophen-2yl)acrylate $(67.3 \mathrm{mg}, 0.400 \mathrm{mmol}),\left[\operatorname{Ir}(\mathrm{Fppy})_{2}(\mathrm{dtbpy})\left(\mathrm{PF}_{6}\right)\right](3.8 \mathrm{mg}, 0.004 \mathrm{mmol})$, styrene $(0.23$ $\mathrm{mL}, 2.00 \mathrm{mmol}), \mathrm{EtAlCl}_{2}(40 \mu \mathrm{L}, 0.040 \mathrm{mmol}), \mathrm{CH}_{2} \mathrm{Cl}_{2}(13.5 \mathrm{~mL})$, and an irradiation time of 16 h. The crude material resulted in a 2.3:1 mixture of diastereomers that was purified by flash chromatography ( $\mathrm{SiO}_{2}, 4 \%$ EtOAc:hexanes). Combined yield: $87.1 \mathrm{mg}$ of a clear oil (80\%, 0.320 mmol). All spectroscopic data match those reported above.

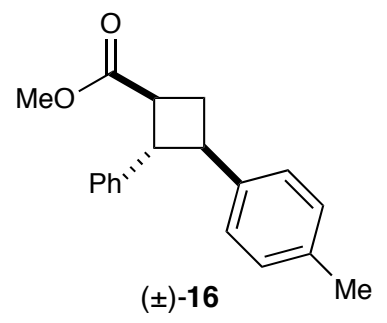

Methyl $\left(1 S^{*}, 2 S^{*}, 3 R^{*}\right)$-2-phenyl-3-(p-tolyl)cyclobutane-1-carboxylate $(16)$. The title compound was prepared according to General Procedure D using methyl cinnamate (64.9 mg, $0.400 \mathrm{mmol}),\left[\operatorname{Ir}(\mathrm{ppy})_{2}(\mathrm{dtbpy})\left(\mathrm{PF}_{6}\right)\right](3.7 \mathrm{mg}, 0.004 \mathrm{mmol}), 1$-methyl-4-vinylbenzene $(0.26 \mathrm{~mL}$, $2.00 \mathrm{mmol}), \mathrm{EtAlCl}_{2}(40 \mu \mathrm{L}, 0.040 \mathrm{mmol}), \mathrm{CH}_{2} \mathrm{Cl}_{2}(13.5 \mathrm{~mL})$, and an irradiation time of $14 \mathrm{~h}$. The crude material resulted in a 2:1 mixture of diastereomers that was purified by flash chromatography $\left(\mathrm{SiO}_{2}, 5 \% \mathrm{Et}_{2} \mathrm{O}\right.$ :pentane). Combined yield: $90.8 \mathrm{mg}$ of a clear oil $(81 \%, 0.324$ mmol). All spectroscopic data match those reported above. 


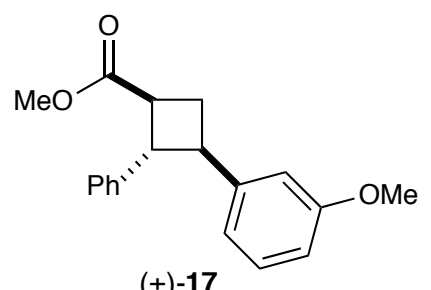

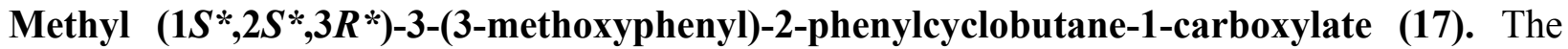
title compound was prepared according to General Procedure D using methyl cinnamate (64.9 $\mathrm{mg}, 0.400 \mathrm{mmol}),\left[\operatorname{Ir}(\mathrm{Fppy})_{2}(\mathrm{dtbpy})\left(\mathrm{PF}_{6}\right)\right]$ (3.8 mg, $\left.0.004 \mathrm{mmol}\right), 3$-vinylanisole $(0.28 \mathrm{~mL}, 2.00$ mmol), EtAlCl $2(40 \mu \mathrm{L}, 0.040 \mathrm{mmol}), \mathrm{CH}_{2} \mathrm{Cl}_{2}(13.5 \mathrm{~mL})$, and an irradiation time of $16 \mathrm{~h}$. The crude material resulted in a 2.1:1 mixture of diastereomers that was purified by flash chromatography $\left(\mathrm{SiO}_{2}, 6 \%\right.$ EtOAc:hexanes). Combined yield: $109.9 \mathrm{mg}$ of a clear oil $(93 \%$, $0.371 \mathrm{mmol})$. All spectroscopic data match those reported above.

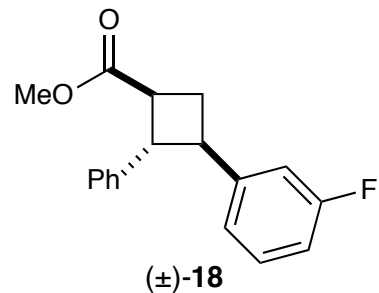

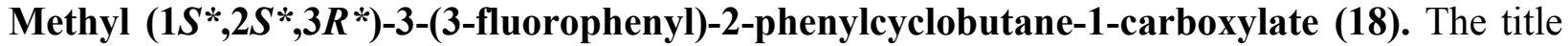
compound was prepared according to General Procedure D using methyl cinnamate (64.9 mg, $0.400 \mathrm{mmol}),\left[\operatorname{Ir}(\mathrm{ppy})_{2}(\mathrm{dtbpy})\left(\mathrm{PF}_{6}\right)\right](3.7 \mathrm{mg}, 0.004 \mathrm{mmol}), 1$-fluoro-3-vinylbezne $(0.24 \mathrm{~mL}$, $2.00 \mathrm{mmol}), \mathrm{EtAlCl}_{2}(40 \mu \mathrm{L}, 0.040 \mathrm{mmol}), \mathrm{CH}_{2} \mathrm{Cl}_{2}(13.5 \mathrm{~mL})$, and an irradiation time of $15 \mathrm{~h}$. The crude material resulted in a 2:1 mixture of diastereomers that was purified by flash chromatography $\left(\mathrm{SiO}_{2}, 5 \% \mathrm{Et}_{2} \mathrm{O}\right.$ :pentane). Combined yield: $99.8 \mathrm{mg}$ of a clear oil $(88 \%, 0.351$ mmol). All spectroscopic data match those reported above. 


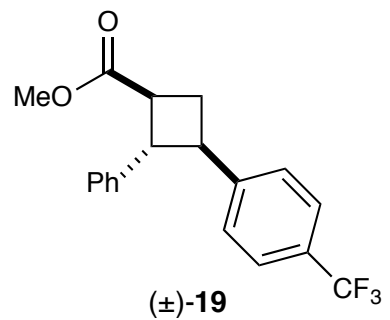

Methyl $\left(1 S^{*}, 2 S^{*}, 3 R^{*}\right)$-2-phenyl-3-(4-(trifluoromethyl)phenyl)cyclobutane-1-carboxylate (19). The title compound was prepared according to General Procedure D using methyl cinnamate $(64.9 \mathrm{mg}, \quad 0.400 \mathrm{mmol}), \quad\left[\operatorname{Ir}(\mathrm{Fppy})_{2}(\mathrm{dtbpy})\left(\mathrm{PF}_{6}\right)\right] \quad(3.8 \mathrm{mg}, \quad 0.004 \mathrm{mmol}), \quad 4-$ (trifluoromethyl)styrene $(0.30 \mathrm{~mL}, 2.00 \mathrm{mmol}), \mathrm{EtAlCl}_{2}(40 \mu \mathrm{L}, 0.040 \mathrm{mmol}), \mathrm{CH}_{2} \mathrm{Cl}_{2}(13.5$ $\mathrm{mL}$ ), and an irradiation time of $16 \mathrm{~h}$. The crude material resulted in a 2.5:1 mixture of diastereomers that was purified by flash chromatography $\left(\mathrm{SiO}_{2}, 6 \%\right.$ EtOAc:hexanes). Combined yield: $122.0 \mathrm{mg}$ of a clear oil $(91 \%, 0.365 \mathrm{mmol})$. All spectroscopic data match those reported above.

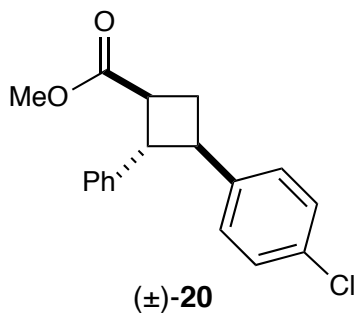

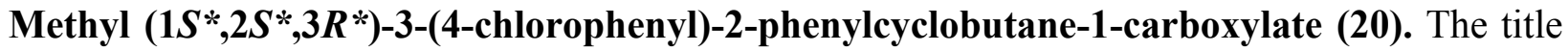
compound was prepared according to General Procedure D using methyl cinnamate $(64.9 \mathrm{mg}$, $0.400 \mathrm{mmol}),\left[\operatorname{Ir}(\mathrm{ppy})_{2}(\mathrm{dtbpy})\left(\mathrm{PF}_{6}\right)\right](3.7 \mathrm{mg}, 0.004 \mathrm{mmol}), 1$-chloro-4-vinylbenzne $(0.24 \mathrm{~mL}$, $2.00 \mathrm{mmol}), \mathrm{EtAlCl}_{2}(40 \mu \mathrm{L}, 0.040 \mathrm{mmol}), \mathrm{CH}_{2} \mathrm{Cl}_{2}(13.5 \mathrm{~mL})$, and an irradiation time of $15 \mathrm{~h}$. The crude material resulted in a 2:1 mixture of diastereomers that was purified by flash chromatography $\left(\mathrm{SiO}_{2}, 5 \% \mathrm{Et}_{2} \mathrm{O}\right.$ :pentane). Combined yield: $111.1 \mathrm{mg}$ of a clear oil $(92 \%, 0.369$ mmol). All spectroscopic data match those reported above. 


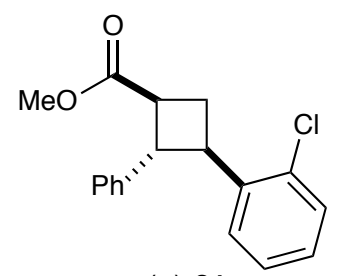

$( \pm)-21$

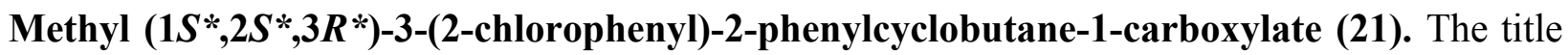
compound was prepared according to General Procedure D using methyl cinnamate (64.9 mg, $0.400 \mathrm{mmol}),\left[\operatorname{Ir}(\mathrm{Fppy})_{2}(\mathrm{dtbpy})\left(\mathrm{PF}_{6}\right)\right](3.8 \mathrm{mg}, 0.004 \mathrm{mmol})$, 2-chlorostyrene $(0.26 \mathrm{~mL}, 2.00$ mmol), EtAlCl $2(40 \mu \mathrm{L}, 0.040 \mathrm{mmol}), \mathrm{CH}_{2} \mathrm{Cl}_{2}(13.5 \mathrm{~mL})$, and an irradiation time of $16 \mathrm{~h}$. The crude material resulted in a 2.5:1 mixture of diastereomers that was purified by flash chromatography $\left(\mathrm{SiO}_{2}, 4 \%\right.$ EtOAc:hexanes). Combined yield: $112.3 \mathrm{mg}$ of a clear oil $(93 \%$, $0.373 \mathrm{mmol})$. All spectroscopic data match those reported above.

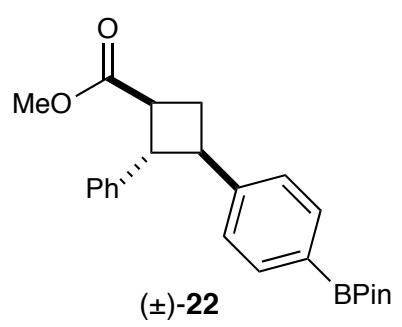

\section{Methyl $\quad\left(1 S^{*}, 2 S *, 3 R^{*}\right)-2-p h e n y l-3-(4-(4,4,5,5$-tetramethyl-1,3,2-dioxaborolan-2-yl)phenyl)}

cyclo-butane-1-carboxylate (22). The title compound was prepared according to General Procedure D using methyl cinnamate $(64.9 \mathrm{mg}, 0.400 \mathrm{mmol}),\left[\operatorname{Ir}(\mathrm{ppy})_{2}(\mathrm{dtbpy})\left(\mathrm{PF}_{6}\right)\right](3.7 \mathrm{mg}$, $0.004 \mathrm{mmol}$ ), 4-vinylphenylboronic acid (460.2 mg, $2.00 \mathrm{mmol}), \mathrm{EtAlCl}_{2}(40 \mu \mathrm{L}, 0.040 \mathrm{mmol})$, $\mathrm{CH}_{2} \mathrm{Cl}_{2}(13.5 \mathrm{~mL})$, and an irradiation time of $48 \mathrm{~h}$. The crude material resulted in a 2:1 mixture of diastereomers that was purified by flash chromatography $\left(\mathrm{SiO}_{2}\right.$, Gradient $2.5 \%$ to $5 \%$ $\mathrm{Et}_{2} \mathrm{O}$ :pentane). Combined yield: $83.2 \mathrm{mg}$ of a clear oil $(63 \%, 0.212 \mathrm{mmol})$. All spectroscopic data match those reported above. 


\section{Asymmetric Synthesis of Norlignan Natural Product 3}

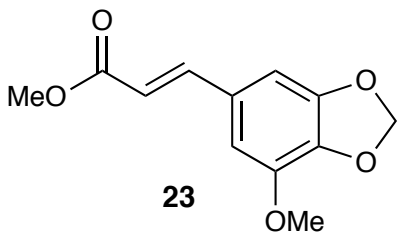

Methyl (E)-3-(7-methoxybenzo[d][1,3]dioxol-5-yl)acrylate (23). The title compound was prepared according to General Procedure B using 5-methoxypiperonal (1.00 g, $5.57 \mathrm{mmol})$, methyl (triphenylphosphoranylidene)acetate $(2.79 \mathrm{~g}, 8.35 \mathrm{mmol})$, and $\mathrm{CH}_{2} \mathrm{Cl}_{2}(28 \mathrm{~mL})$. The crude material was purified by flash chromatography $\left(\mathrm{SiO}_{2}, 15 \rightarrow 20 \%\right.$ EtOAc:hexanes $)$ to afford methyl (E)-3-(7-methoxybenzo[d][1,3]dioxol-5-yl)acrylate $(1.23 \mathrm{~g}, 93 \%)$ as a white solid (mp 100-101 $\left.{ }^{\circ} \mathrm{C}\right) .{ }^{1} \mathrm{H} \mathrm{NMR}\left(\mathrm{CDCl}_{3}, 500 \mathrm{MHz}\right) \delta 7.57(\mathrm{~d}, J=15.9 \mathrm{~Hz}, 1 \mathrm{H}), 6.75(\mathrm{~s}, 1 \mathrm{H}), 6.70(\mathrm{~s}, 1 \mathrm{H})$, $6.28(\mathrm{~d}, J=15.9 \mathrm{~Hz}, 1 \mathrm{H}), 6.02(\mathrm{~s}, 2 \mathrm{H}), 3.92(\mathrm{~s}, 3 \mathrm{H}), 3.80(\mathrm{~s}, 3 \mathrm{H}) ;{ }^{13} \mathrm{C} \mathrm{NMR}\left(126 \mathrm{MHz}, \mathrm{CDCl}_{3}\right) \delta$ $167.5,149.3,144.6,143.7,137.4,129.2,116.3,109.1,102.0,101.3,56.6,51.7$; IR (thin film) $v$ $3070,3004,2950,2904,2845,1705,1636,1623,1603,1508,1449,1429,1277,1168,1135$, 1093, 1041, 978, 924, $828 \mathrm{~cm}^{-1}$; HRMS (ESI) $m / \mathrm{z}$ calcd for $\mathrm{C}_{12} \mathrm{H}_{12} \mathrm{O}_{5} \mathrm{H}(\mathrm{M}+\mathrm{H})^{+} 237.0758$, found 237.0754 .

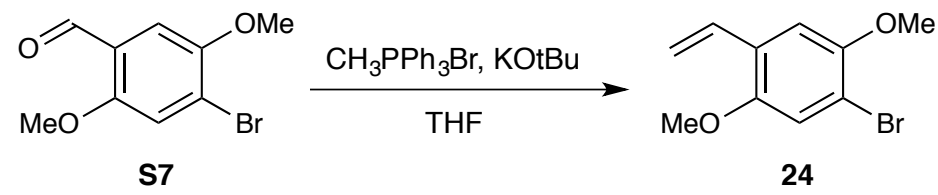

1-Bromo-2,5-dimethoxy-4-vinylbenzene (24). The following procedure was adapted from Kamada et $a l .{ }^{8}$ To a solution of methyltriphenylphosphonium bromide $(9.37 \mathrm{~g}, 26.2 \mathrm{mmol})$ in THF $(100 \mathrm{~mL})$ at $0{ }^{\circ} \mathrm{C}$ was added potassium tert-butoxide $(2.94 \mathrm{~g}, 26.2 \mathrm{mmol})$ in one portion. After thirty minutes, the reaction mixture was cool to $-78{ }^{\circ} \mathrm{C}$ and 4-bromo-2,5dimethoxybenzaldehyde (4.95 g, $20.2 \mathrm{mmol})$ in THF (35 mL) was added dropwise via syringe. The transfer was completed with an additional two portions of THF $(5 \mathrm{~mL})$. After $1 \mathrm{~h}$, the 
reaction mixture was allowed to warm to room temperature. After $1 \mathrm{~h}$ at room temperature, water $(100 \mathrm{~mL})$ was added to the reaction mixture, followed by $\mathrm{Et}_{2} \mathrm{O}(200 \mathrm{~mL})$. The aqueous layer was extracted two times with $\mathrm{Et}_{2} \mathrm{O}(100 \mathrm{~mL})$. The combined organic extracts were washed with water (100 mL), brine $(100 \mathrm{~mL})$, dried over $\mathrm{MgSO}_{4}$, filtered, and concentrated in vacuo. The crude material was purified by flash column chromatography $\left(\mathrm{SiO}_{2}\right.$, Gradient $0 \%$ to $6 \%$ EtOAc:hexanes) to afford the title compound (4.59 g, 94\%). ${ }^{1} \mathrm{H} \mathrm{NMR}\left(\mathrm{CDCl}_{3}, 500 \mathrm{MHz}\right) \delta 7.06$ (s, 1H), $7.02(\mathrm{~s}, 1 \mathrm{H}), 6.97(\mathrm{dd}, J=17.5,11.0 \mathrm{~Hz}, 1 \mathrm{H}), 5.73(\mathrm{dd}, J=17.5,1.0 \mathrm{~Hz}, 1 \mathrm{H}), 5.30$ (dd, $J=11.0,1.0 \mathrm{~Hz}, 1 \mathrm{H}), 3.88(\mathrm{~s}, 3 \mathrm{H}), 3.80(\mathrm{~s}, 3 \mathrm{H}) ;{ }^{13} \mathrm{C} \mathrm{NMR}\left(126 \mathrm{MHz}, \mathrm{CDCl}_{3}\right) \delta 151.2,150.1$, $131.0,126.6,116.6,115.0,111.1,110.1,56.9,56.4$.

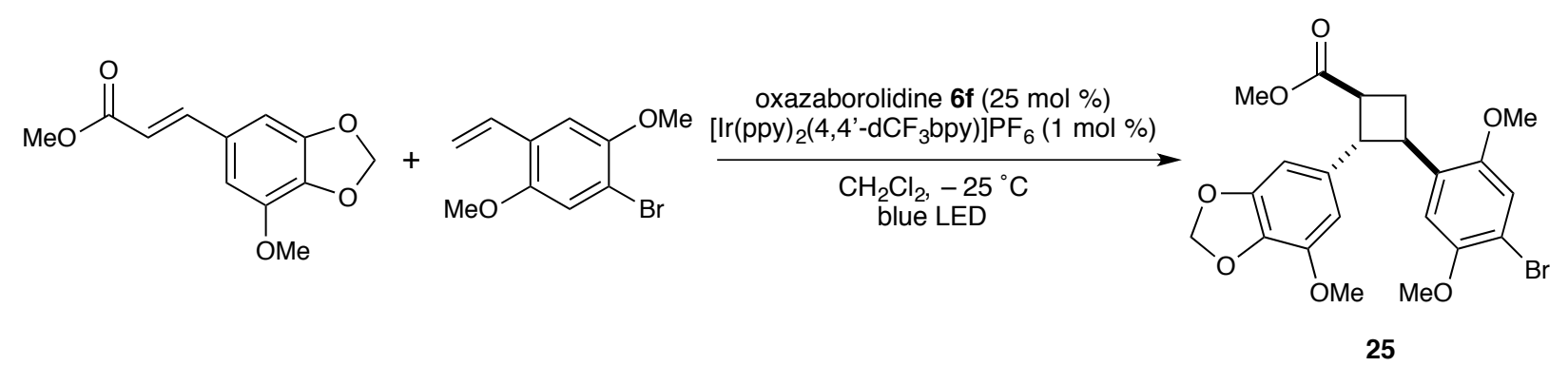

\section{((1R,2R,3S)-3-(4-Bromo-2,5-dimethoxyphenyl)-2-(7-methoxybenzo[d][1,3]dioxol-5-}

yl)cyclobutyl) (2-hydroxyphenyl)methanone (25). The title compound was prepared according to General Procedure C using methyl $(E)-3-(7-m e t h o x y b e n z o[d][1,3]$ dioxol-5-yl)acrylate $(94.5$ mg, $0.400 \mathrm{mmol}$, [Ir(ppy) 2 (4,4'- $\left.\left.\mathrm{dCF}_{3} \mathrm{bpy}\right)\left(\mathrm{PF}_{6}\right)\right]$ (3.8 mg, $\left.0.004 \mathrm{mmol}\right)$, 1-bromo-2,5-dimethoxy4-vinylbenzene (486.2 mg, $2.00 \mathrm{mmol})$, oxazaborolidine $\mathbf{6 f}(1.0 \mathrm{~mL}, 0.100 \mathrm{mmol})$, and $\mathrm{CH}_{2} \mathrm{Cl}_{2}$ $(12.5 \mathrm{~mL})$, an irradiation time of $36 \mathrm{~h}$. The crude material resulted in a 5:1 mixture of diastereomers that was purified by flash chromatography $\left(\mathrm{SiO}_{2}\right.$, Gradient $20 \%$ to $40 \% \mathrm{MTBE}$ : Pentane. Yield: $151.9 \mathrm{mg}$ of a cloudy oil $(80 \%, 0.318 \mathrm{mmol})$; 97\% ee (Daicel CHIRALPAK® OD-H, 5-50\% IPA:hexanes over $13 \mathrm{~min}$, flow rate of $1 \mathrm{~mL} / \mathrm{min} ; \mathrm{t}_{1}=9.31 \mathrm{~min}, \mathrm{t}_{2}=9.93 \mathrm{~min}$ ); 
$[\alpha]_{\mathrm{D}}{ }^{22}=-60.0^{\circ}\left(\mathrm{c}=0.60, \mathrm{CH}_{2} \mathrm{Cl}_{2}\right)$; lit for $93 \%$ ee $[\alpha]_{\mathrm{D}}{ }^{22}=+54.3^{\circ}\left(\mathrm{c}=0.44, \mathrm{CH}_{2} \mathrm{Cl}_{2}\right){ }^{9}$ All spectroscopic data match with those reported previously. ${ }^{9}$

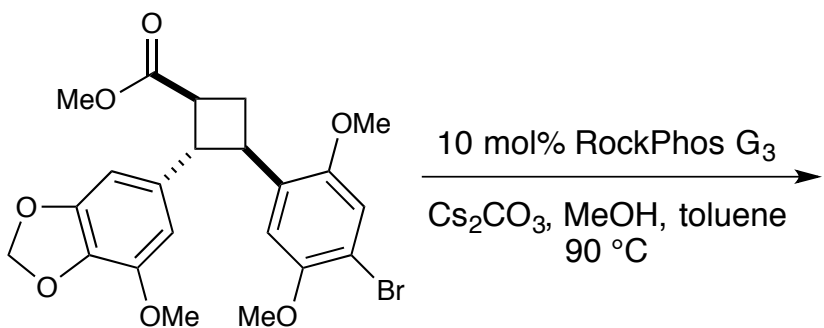

25

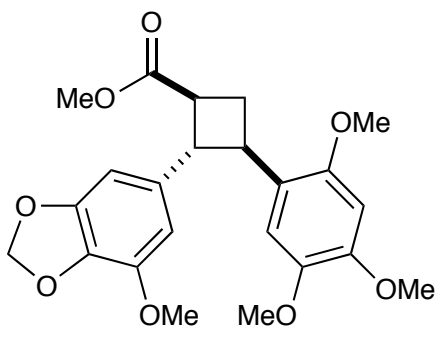

3

Methyl

(1R,2R,3S)-2-(7-methoxybenzo[d][1,3]dioxol-5-yl)-3-(2,4,5-

trimethoxyphenyl)cyclobutane 1-carboxylate (3). A $8 \mathrm{~mL}$ screw cap vial equipped with a magnetic stir bar and was charged with the cyclobutane 25 (1 equiv, $90.0 \mathrm{mg}, 0.188 \mathrm{mmol}, 97 \%$ ee), Cs2CO3 (2 equiv, $133 \mathrm{mg}, 0.376 \mathrm{mmol}$ ), and [(2-di-tertbutylphosphino-3-methoxy-6methyl-2',4',6'-triisopropyl-1,1'-biphenyl)-2-(2-aminobiphenyl)]palladium(II) methanesulfonate (RockPhos Pd G3, 0.1 equiv, $16.0 \mathrm{mg}, 0.0188 \mathrm{mmol}$ ). The vial was sealed with septum, then evacuated and backfilled with nitrogen three times. Methanol ( 5 equiv, $0.940 \mathrm{mmol}, 38 \mu \mathrm{L})$ was added by syringe, followed by toluene $(1.9 \mathrm{~mL})$. The vial was sealed with Teflon cap, and the reaction mixture was stirred at $90{ }^{\circ} \mathrm{C}$ for $24 \mathrm{~h}$. The reaction mixture was then cooled to room temperature, diluted with $5 \mathrm{~mL}$ ethyl acetate, and filtered. The crude reaction mixture was concentrated in vacuo and purified by flash-column chromatography on silica gel (60\% $\mathrm{Et}_{2} \mathrm{O}$ :pentanes) to afford the product $(74.6 \mathrm{mg}, 92 \%, 0.173 \mathrm{mmol})$ as a colorless oil; $97 \%$ ee (Daicel CHIRALPAK ${ }^{\circledR}$ OJ-H, isocratic 40\% EtOH:hexanes over $40 \mathrm{~min}$,flow rate of $4 \mathrm{~mL} / \mathrm{min}$; $\left.\mathrm{t}_{1}=21.3 \mathrm{~min}, \mathrm{t}_{2}=24.5 \mathrm{~min}\right) ;[\alpha]_{\mathrm{D}}^{22}=-33.0^{\circ}\left(\mathrm{c}=0.41, \mathrm{CHCl}_{3}\right)$; lit for $92 \%$ ee $[\alpha]_{\mathrm{D}}^{22}=+20.0^{\circ}(\mathrm{c}$ $\left.=0.01, \mathrm{CHCl}_{3}\right)$. All spectroscopic data match with those reported previously. ${ }^{10}$ 
V. HPLC Chromatograms

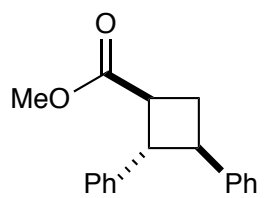

Racemic (Daicel CHIRALCEL ${ }^{\circledR}$ OD-H, 5-50\% IPA:hexanes over $13 \mathrm{~min}, 1 \mathrm{~mL} / \mathrm{min}, 222.0 \mathrm{~nm}$ )

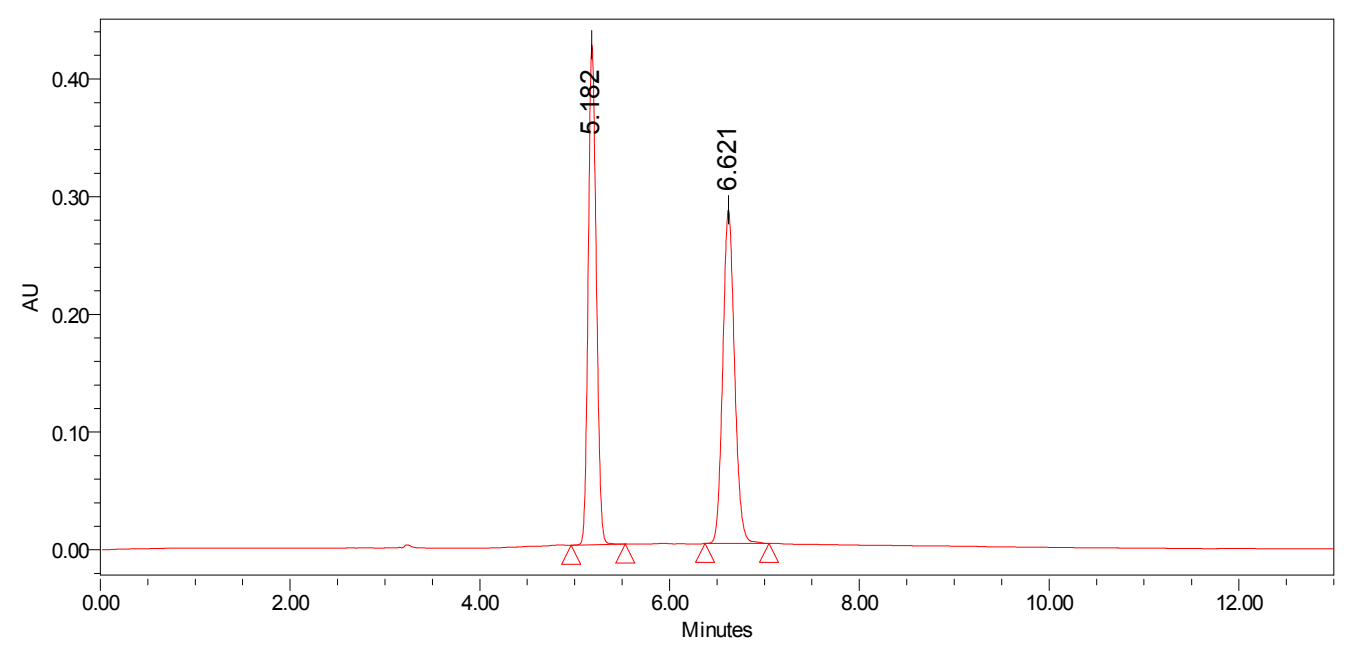

Peak Results

\begin{tabular}{|c|c|c|c|c|}
\hline & RT & Area & Height & \% Area \\
\hline \hline 1 & 5.182 & 2505467 & 424874 & 50.64 \\
\hline 2 & 6.621 & 2442238 & 283495 & 49.36 \\
\hline
\end{tabular}

\section{Enantioenriched}

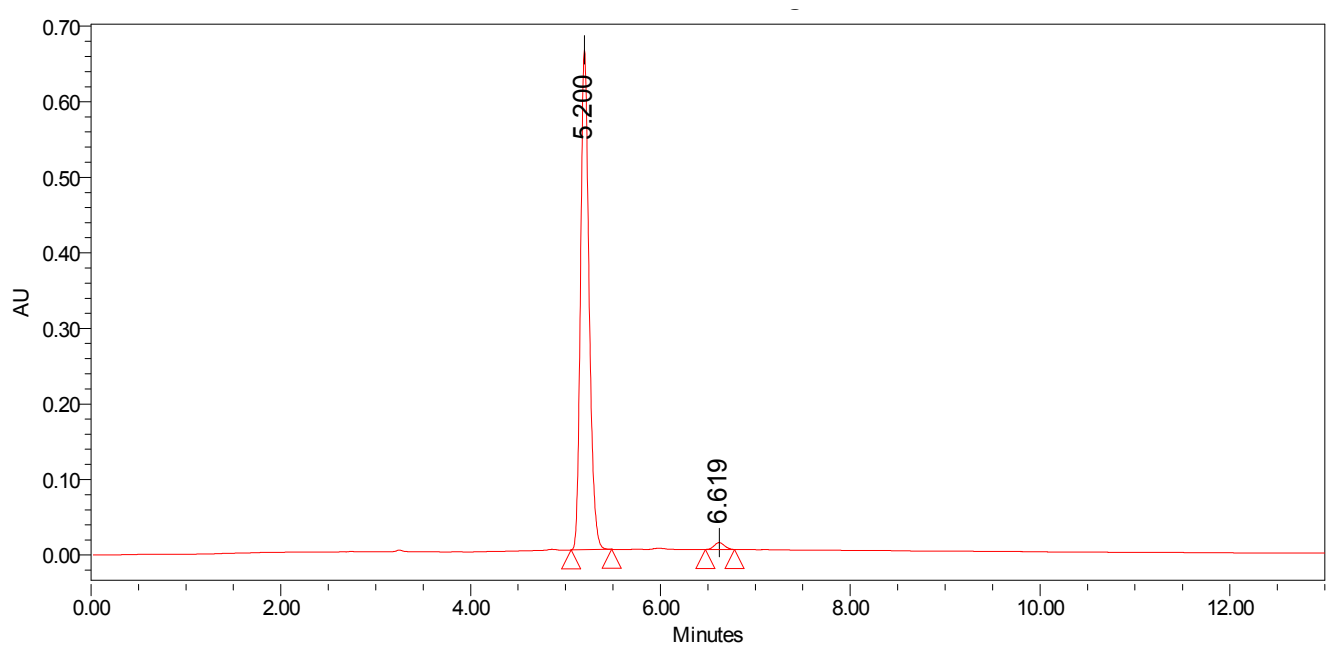

\section{Peak Results}

\begin{tabular}{|c|c|c|c|c|}
\hline & RT & Area & Height & \% Area \\
\hline \hline 1 & 5.200 & 4135532 & 661989 & 98.38 \\
\hline 2 & 6.619 & 68086 & 9061 & 1.62 \\
\hline
\end{tabular}




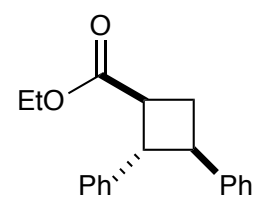

8

Racemic (Daicel CHIRALCEL ${ }^{\circledR}$ OD-H, 5-50\% IPA:hexanes over $13 \mathrm{~min}, 1 \mathrm{~mL} / \mathrm{min}, 222.0 \mathrm{~nm}$ )

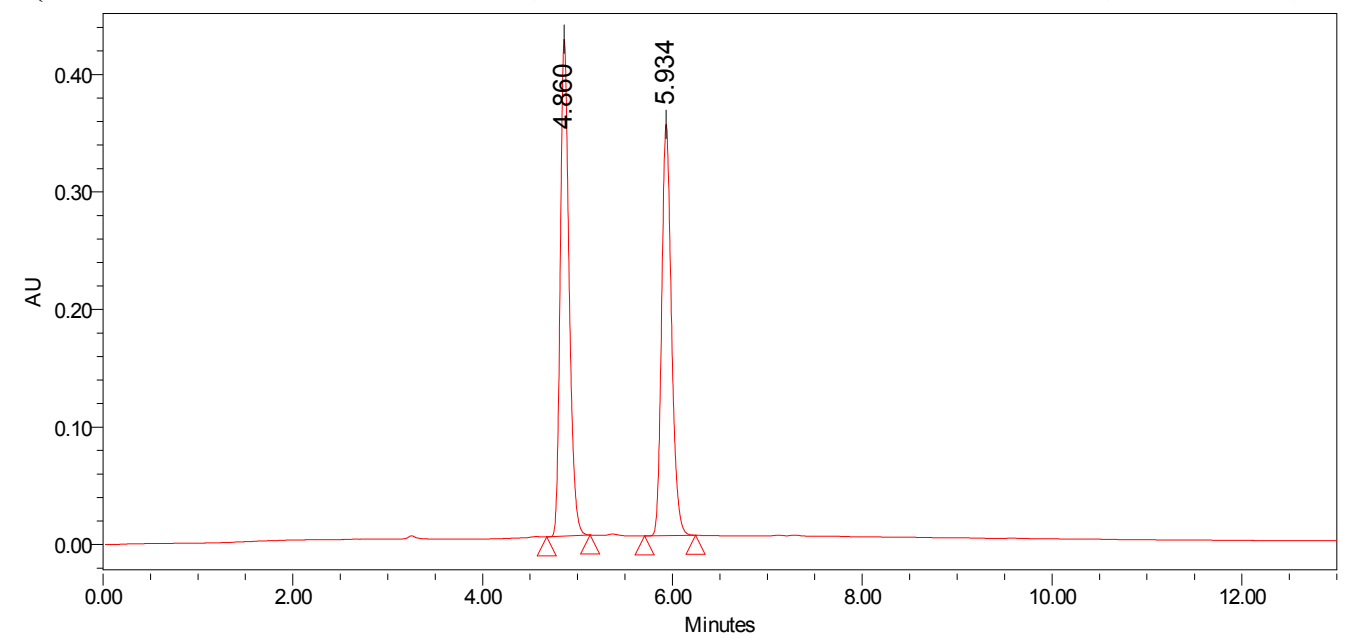

Peak Results

\begin{tabular}{|c|c|c|c|c|}
\hline & RT & Area & Height & \% Area \\
\hline \hline 1 & 4.860 & 2741451 & 423072 & 52.09 \\
\hline 2 & 5.934 & 2521587 & 349914 & 47.91 \\
\hline
\end{tabular}

\section{Enantioenriched}

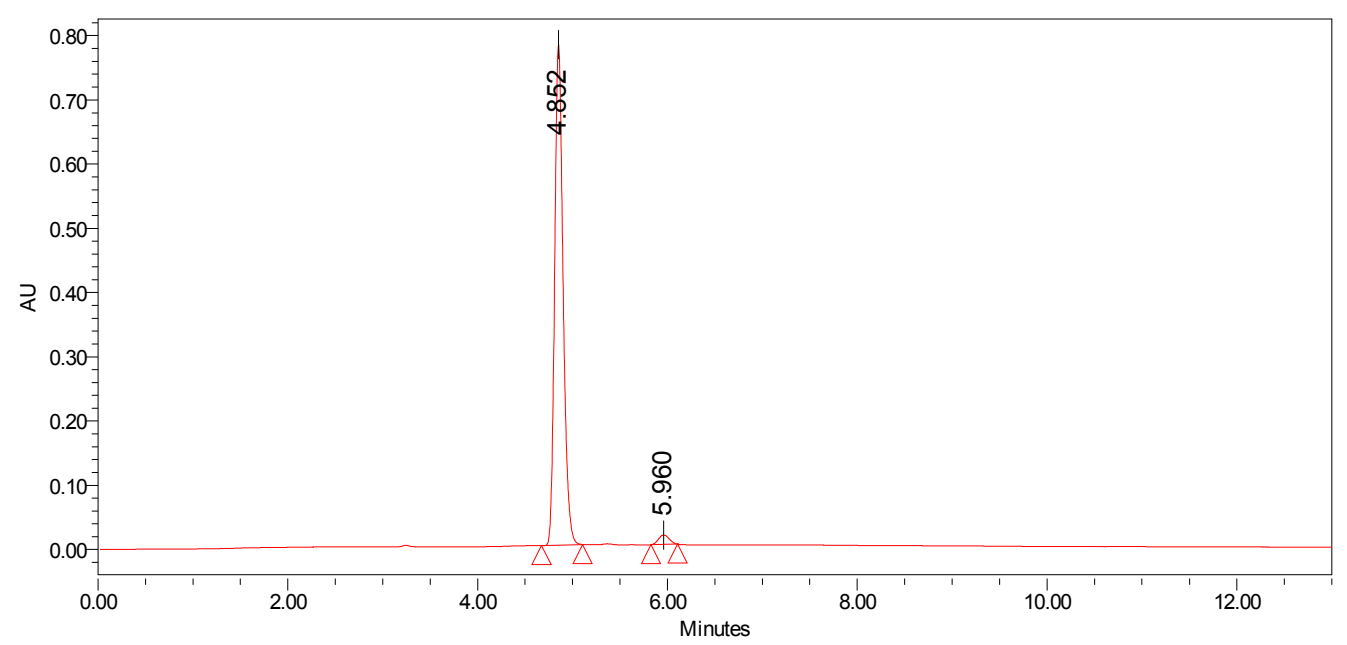

Peak Results

\begin{tabular}{|c|c|c|c|c|}
\hline & RT & Area & Height & \% Area \\
\hline \hline 1 & 4.852 & 4829024 & 779656 & 97.67 \\
\hline 2 & 5.960 & 115099 & 14688 & 2.33 \\
\hline
\end{tabular}




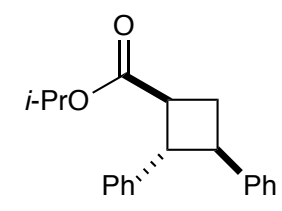

9

Racemic (Daicel CHIRALCEL ${ }^{\circledR}$ OD-H, 5-50\% IPA:hexanes over $13 \mathrm{~min}, 1 \mathrm{~mL} / \mathrm{min}, 222.0 \mathrm{~nm}$ )

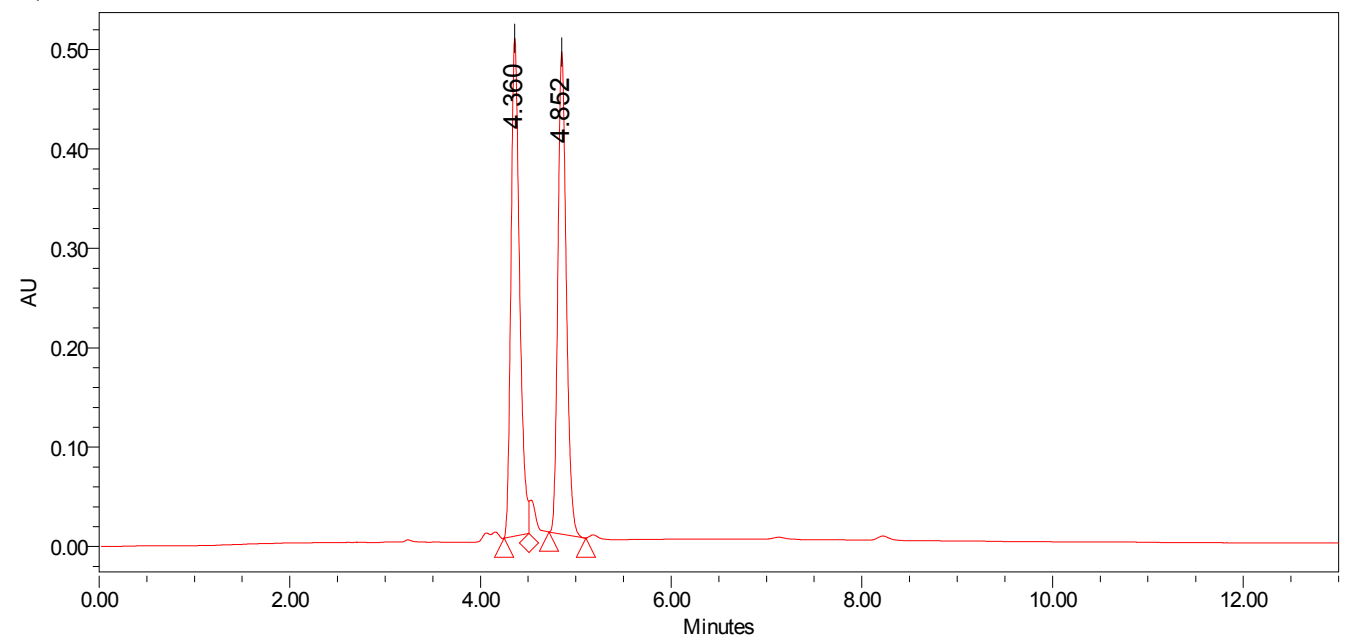

Peak Results

\begin{tabular}{|c|c|c|c|c|}
\hline & RT & Area & Height & \% Area \\
\hline \hline 1 & 4.360 & 3143640 & 500909 & 51.36 \\
\hline 2 & 4.852 & 2976642 & 485148 & 48.64 \\
\hline
\end{tabular}

\section{Enantioenriched}

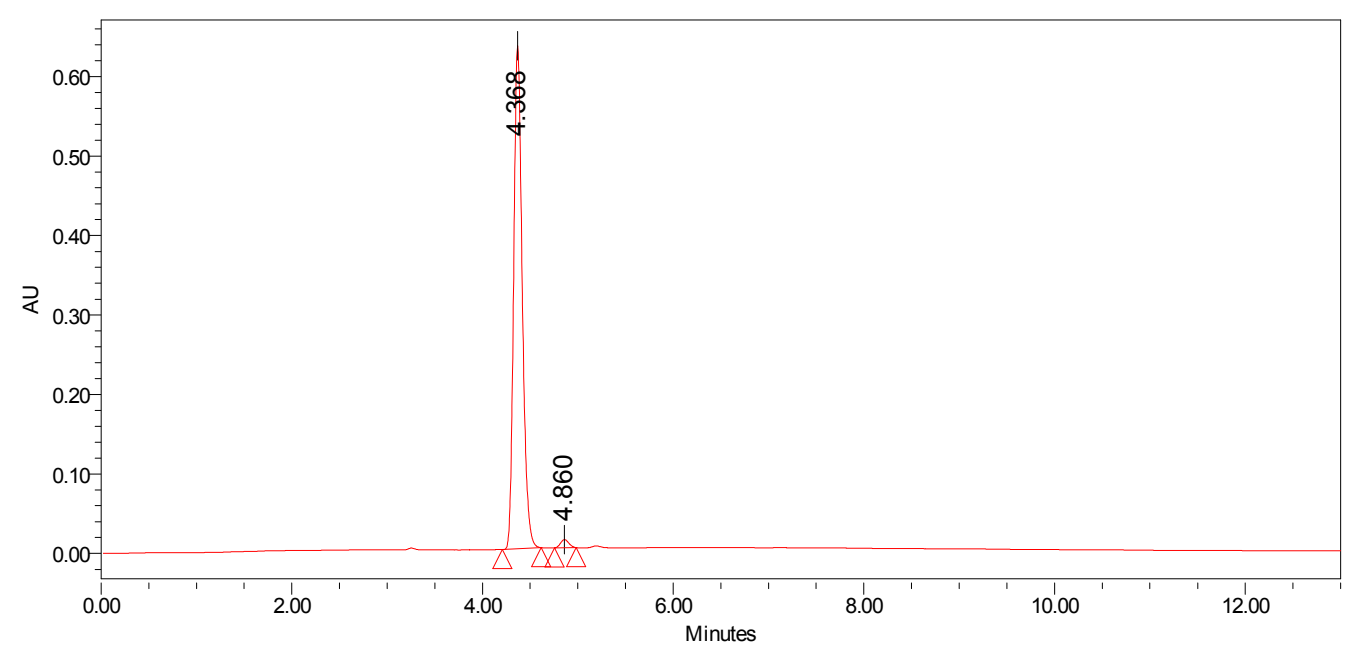

Peak Results

\begin{tabular}{|c|c|c|c|c|}
\hline & RT & Area & Height & \% Area \\
\hline \hline 1 & 4.368 & 3943265 & 633231 & 98.45 \\
\hline 2 & 4.860 & 61916 & 10305 & 1.55 \\
\hline
\end{tabular}




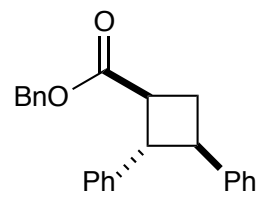

10

Racemic (Daicel CHIRALCEL ${ }^{\circledR}$ OD-H, 5-50\% IPA:hexanes over $13 \mathrm{~min}, 1 \mathrm{~mL} / \mathrm{min}, 222.0 \mathrm{~nm}$ )

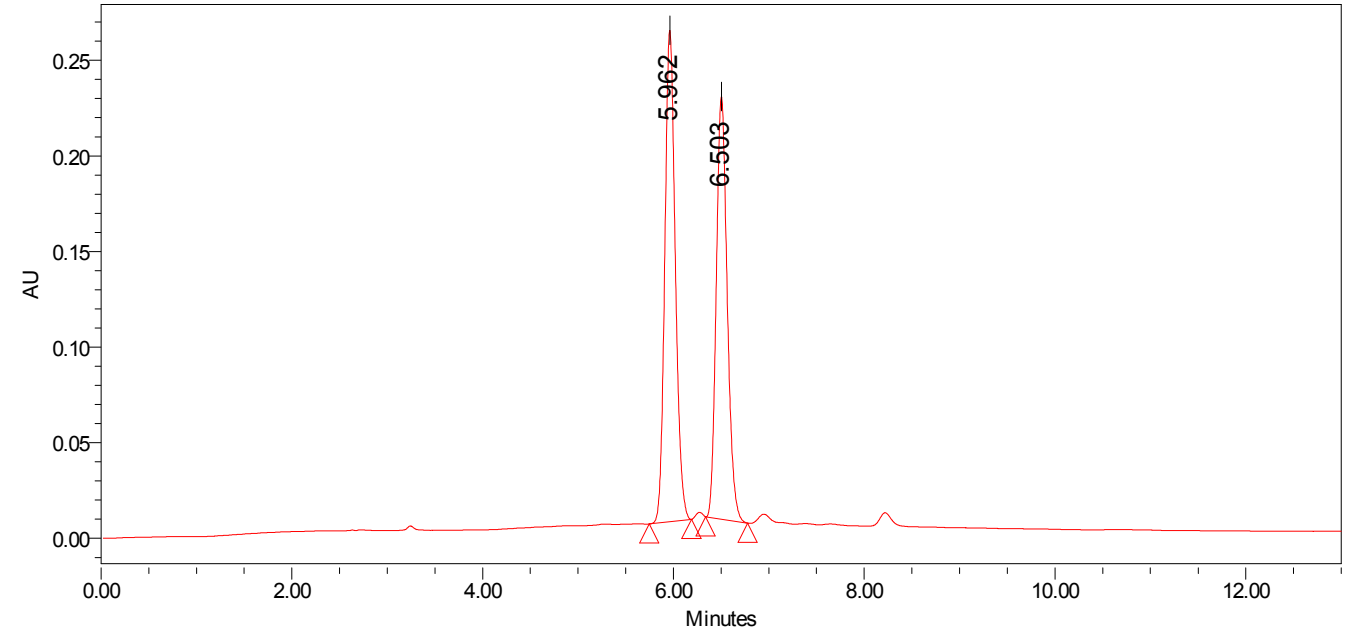

Peak Results

\begin{tabular}{|c|c|c|c|c|}
\hline & RT & Area & Height & \% Area \\
\hline \hline 1 & 5.962 & 1968532 & 257137 & 52.65 \\
\hline 2 & 6.503 & 1770448 & 221110 & 47.35 \\
\hline
\end{tabular}

\section{Enantioenriched}

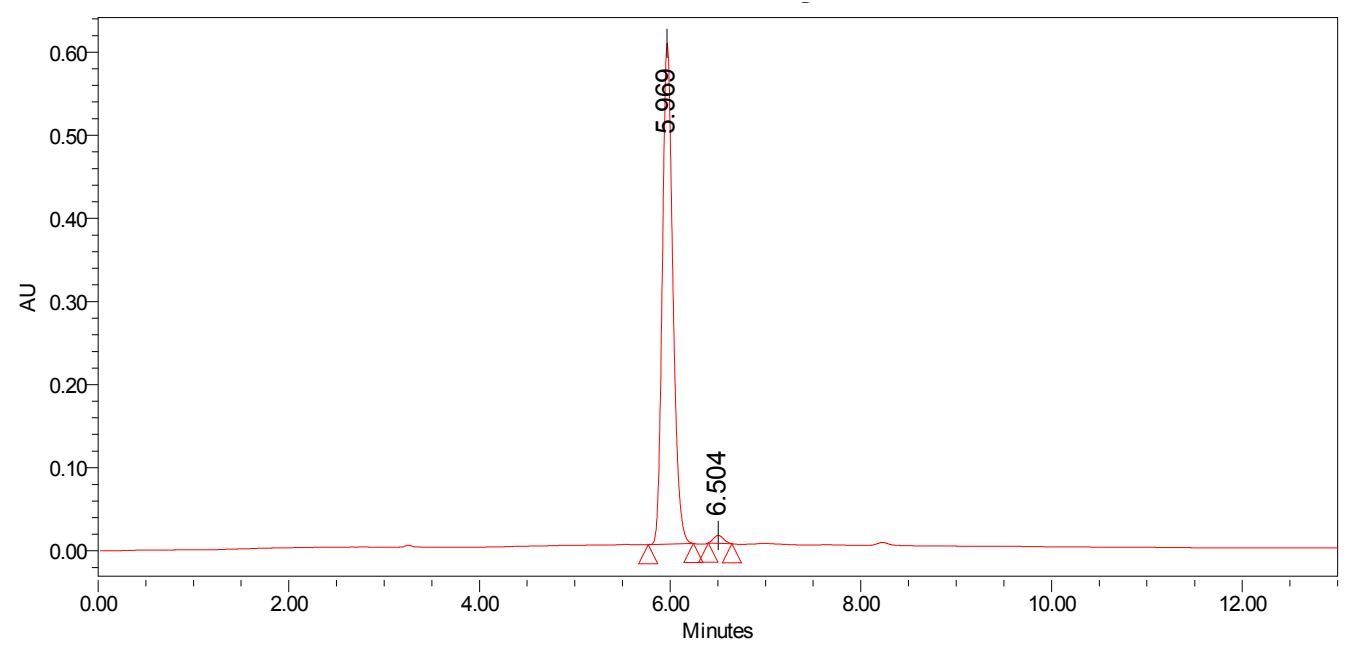

Peak Results

\begin{tabular}{|c|c|c|c|c|}
\hline & RT & Area & Height & \% Area \\
\hline \hline 1 & 5.969 & 4628115 & 602809 & 98.52 \\
\hline 2 & 6.504 & 69524 & 9568 & 1.48 \\
\hline
\end{tabular}




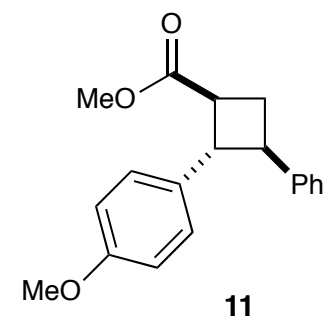

Racemic (Daicel CHIRALCEL ${ }^{\circledR}$ OD-H, 5-50\% IPA:hexanes over $13 \mathrm{~min}, 1 \mathrm{~mL} / \mathrm{min}, 222.0 \mathrm{~nm}$ )

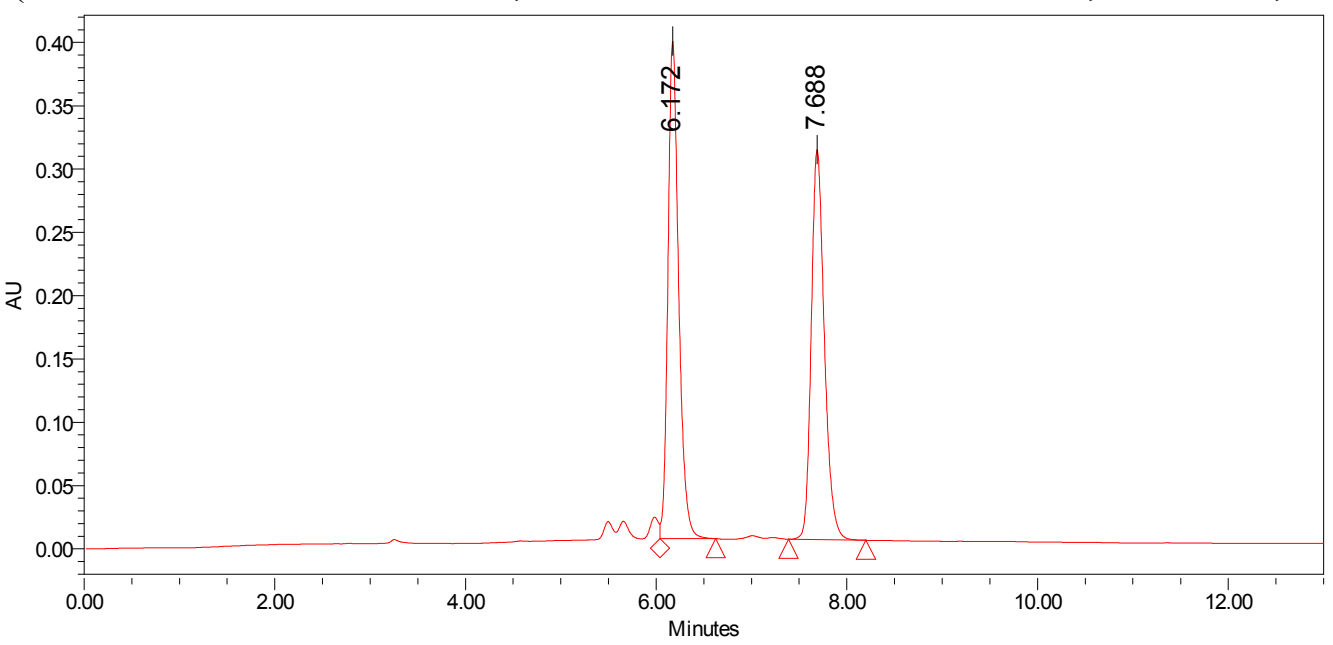

Peak Results

\begin{tabular}{|c|c|c|c|c|}
\hline & RT & Area & Height & \% Area \\
\hline \hline 1 & 6.172 & 3064029 & 393182 & 51.03 \\
\hline 2 & 7.688 & 2939879 & 308173 & 48.97 \\
\hline
\end{tabular}

\section{Enantioenriched}

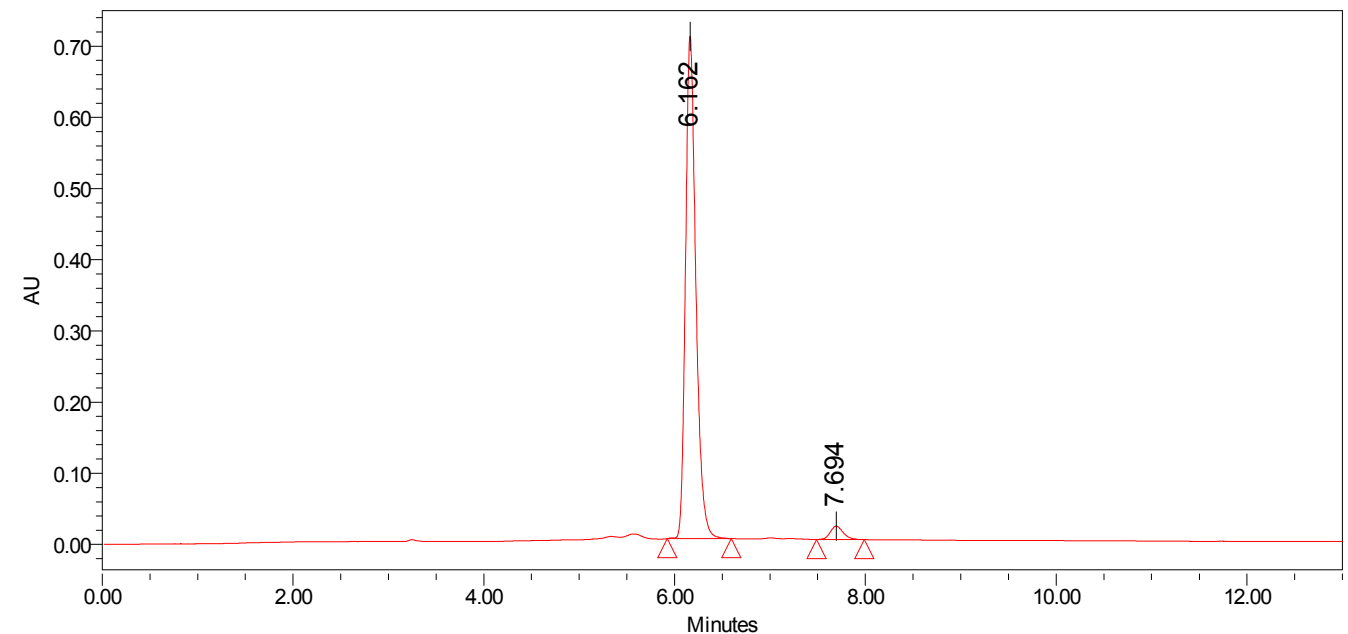

Peak Results

\begin{tabular}{|c|c|c|c|c|}
\hline & RT & Area & Height & \% Area \\
\hline \hline 1 & 6.162 & 5299039 & 705817 & 96.80 \\
\hline 2 & 7.694 & 174918 & 18695 & 3.20 \\
\hline
\end{tabular}




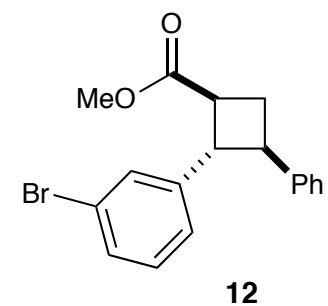

Racemic (Daicel CHIRALCEL ${ }^{\circledR}$ OD-H, 5-50\% IPA:hexanes over $13 \mathrm{~min}, 1 \mathrm{~mL} / \mathrm{min}, 222.0 \mathrm{~nm}$ )

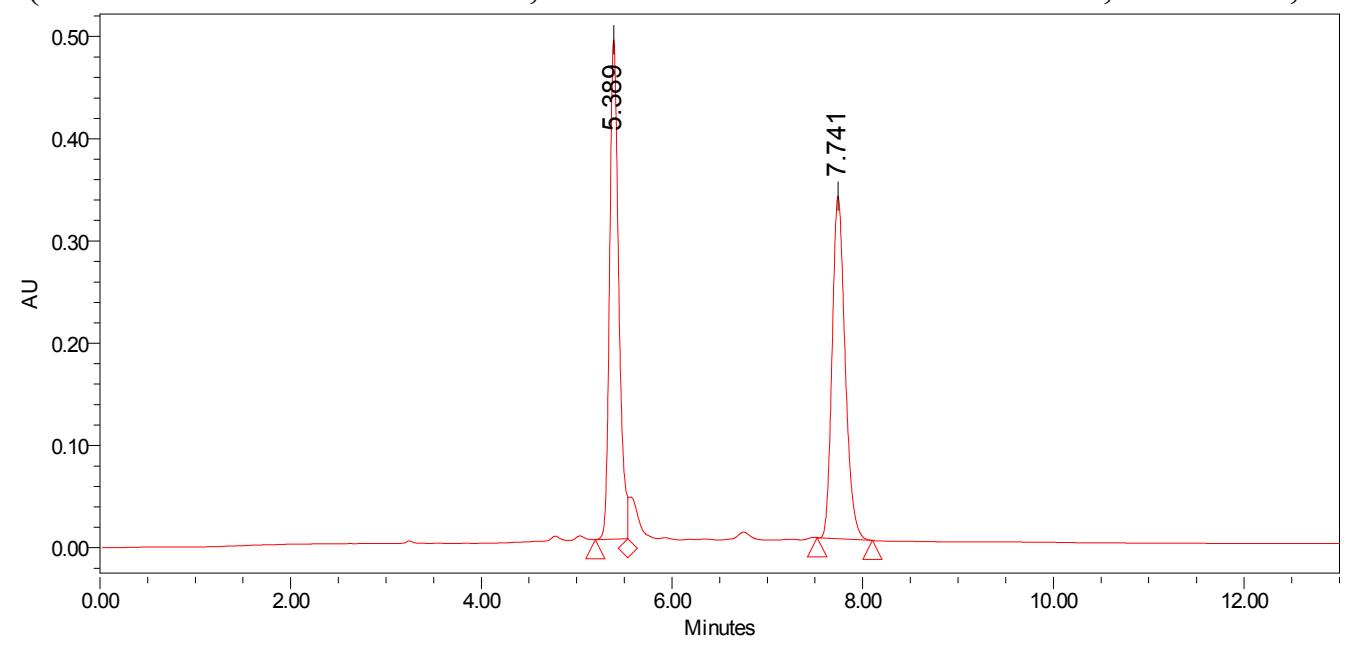

Peak Results

\begin{tabular}{|c|c|c|c|c|}
\hline & RT & Area & Height & \% Area \\
\hline \hline 1 & 5.389 & 3238774 & 488643 & 50.50 \\
\hline 2 & 7.741 & 3174993 & 335236 & 49.50 \\
\hline
\end{tabular}

\section{Enantioenriched}

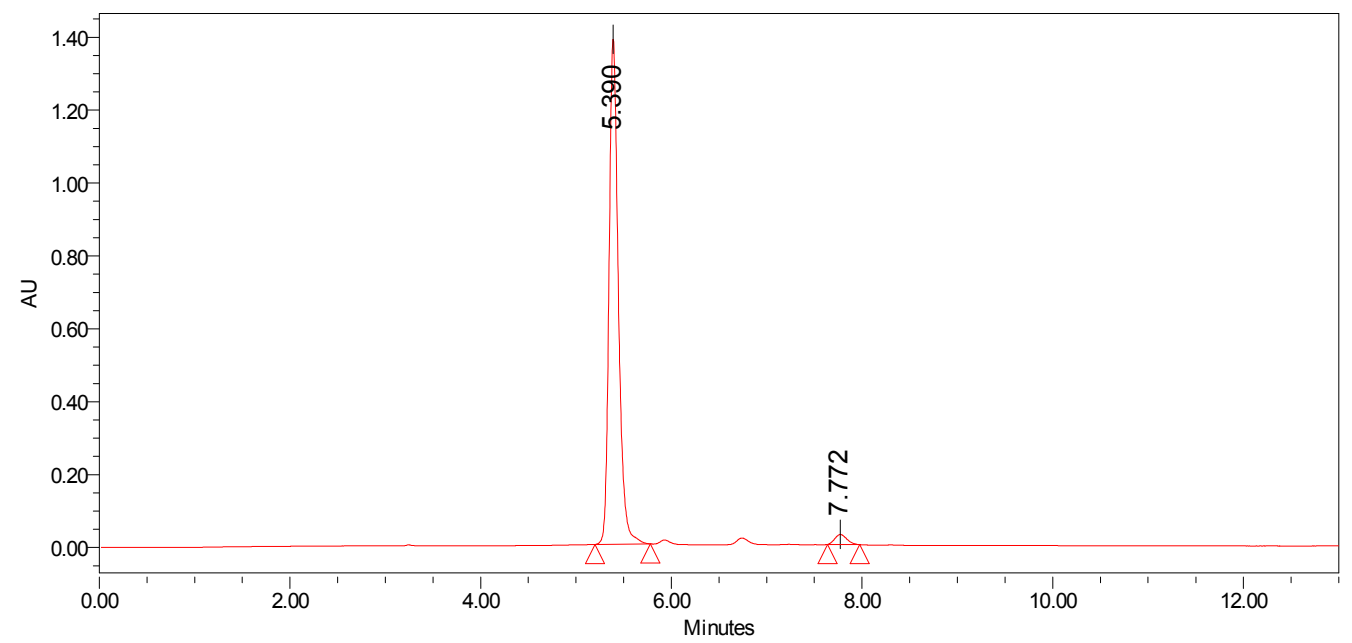

\section{Peak Results}

\begin{tabular}{|c|c|c|c|c|}
\hline & RT & Area & Height & \% Area \\
\hline \hline 1 & 5.390 & 9362557 & 1385896 & 97.49 \\
\hline 2 & 7.772 & 240671 & 27374 & 2.51 \\
\hline
\end{tabular}




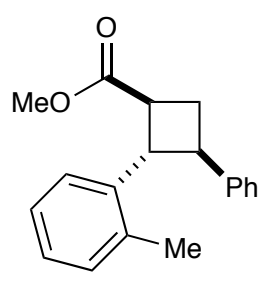

13

Racemic (Daicel CHIRALCEL ${ }^{\circledR}$ OD-H, 5-50\% IPA:hexanes over $13 \mathrm{~min}, 1 \mathrm{~mL} / \mathrm{min}, 222.0 \mathrm{~nm}$ )

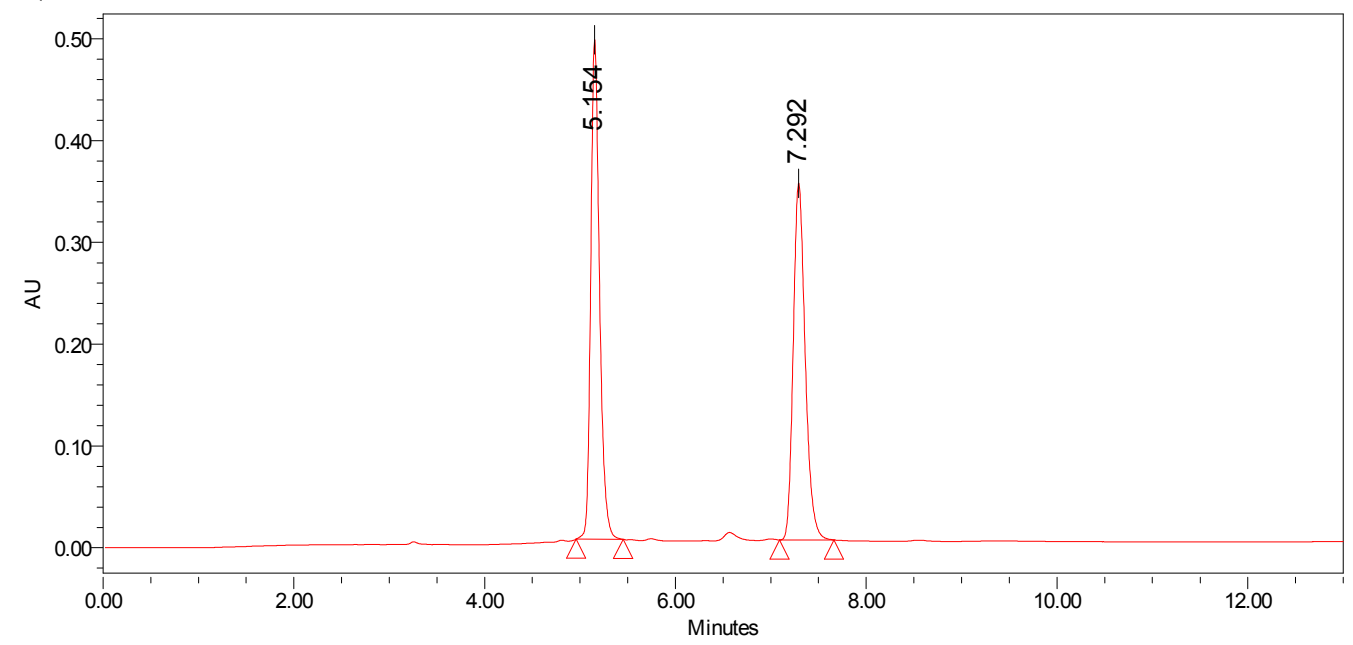

Peak Results

\begin{tabular}{|c|c|c|c|c|}
\hline & RT & Area & Height & \% Area \\
\hline \hline 1 & 5.154 & 3135303 & 491017 & 50.68 \\
\hline 2 & 7.292 & 3051152 & 350664 & 49.32 \\
\hline
\end{tabular}

\section{Enantioenriched}

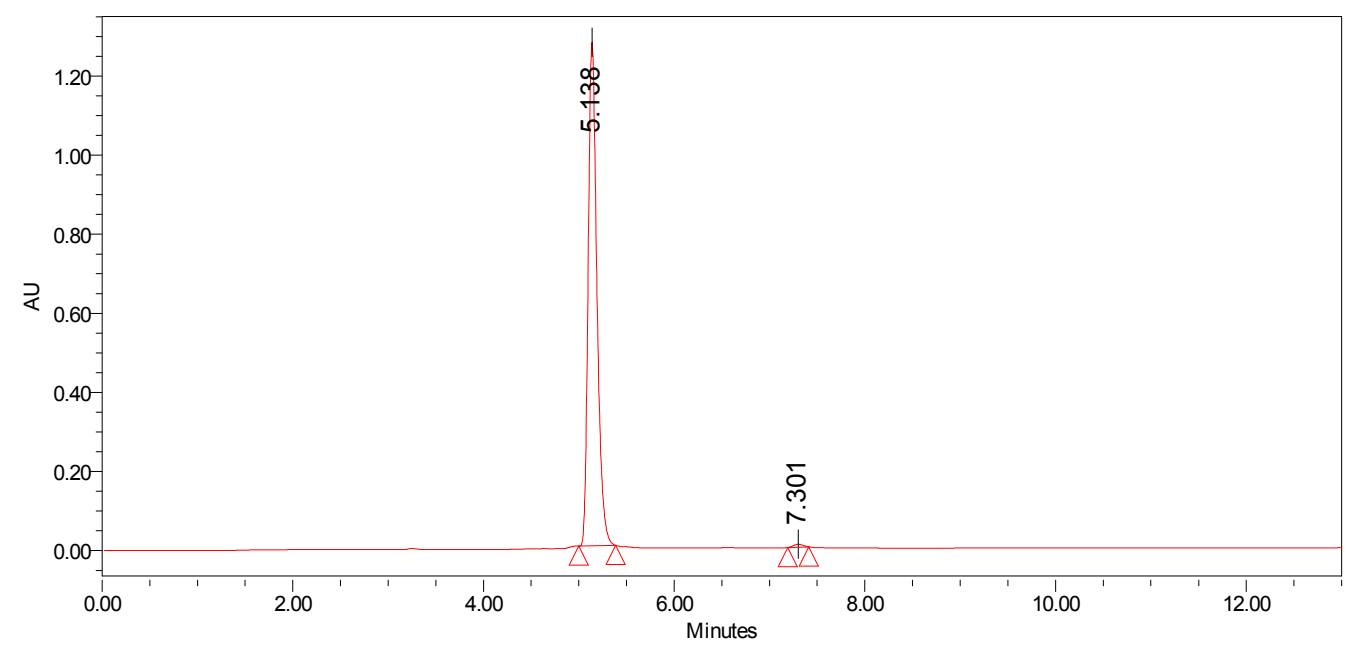

Peak Results

\begin{tabular}{|c|c|c|c|c|}
\hline & RT & Area & Height & \% Area \\
\hline \hline 1 & 5.138 & 8121765 & 1274227 & 99.37 \\
\hline 2 & 7.301 & 51870 & 7422 & 0.63 \\
\hline
\end{tabular}




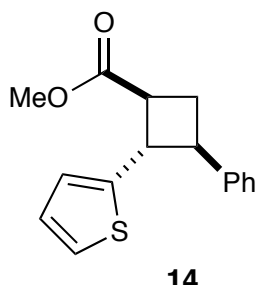

Racemic (Daicel CHIRALCEL ${ }^{\circledR}$ OD-H, 5-50\% IPA:hexanes over $13 \mathrm{~min}, 1 \mathrm{~mL} / \mathrm{min}, 222.0 \mathrm{~nm}$ )

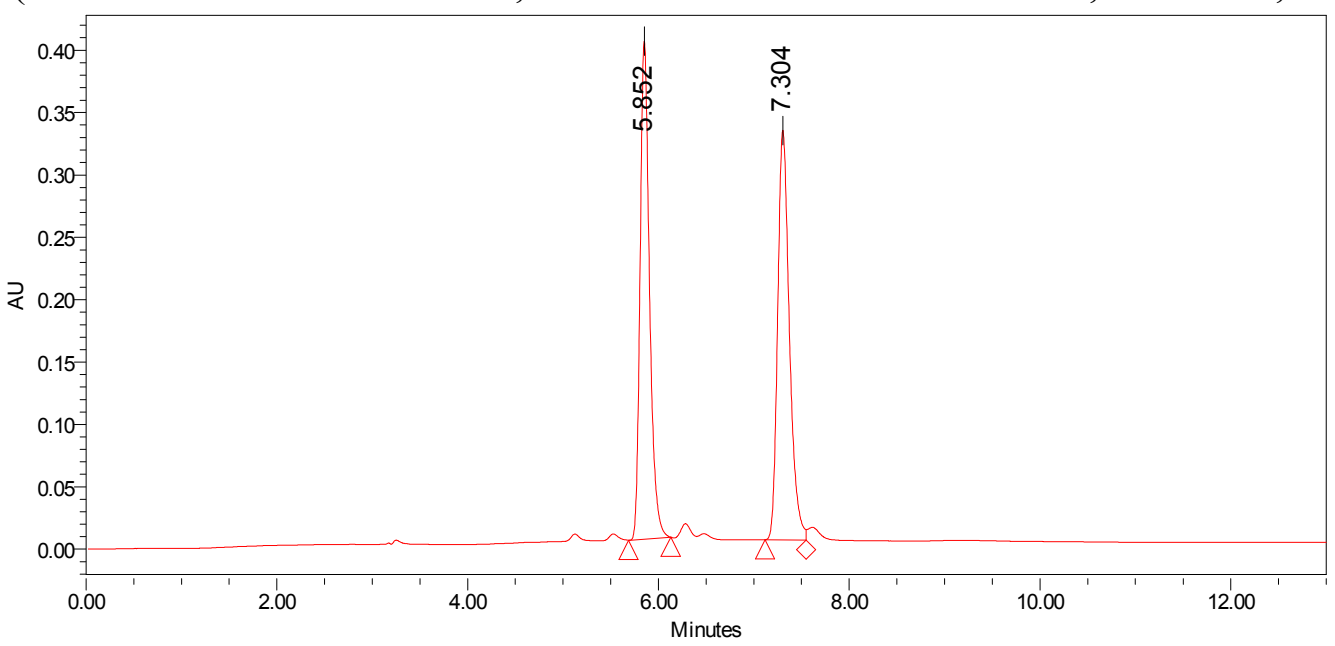

Peak Results

\begin{tabular}{|c|c|c|c|c|}
\hline & RT & Area & Height & \% Area \\
\hline \hline 1 & 5.852 & 2739494 & 399438 & 49.42 \\
\hline 2 & 7.304 & 2803356 & 328354 & 50.58 \\
\hline
\end{tabular}

\section{Enantioenriched}

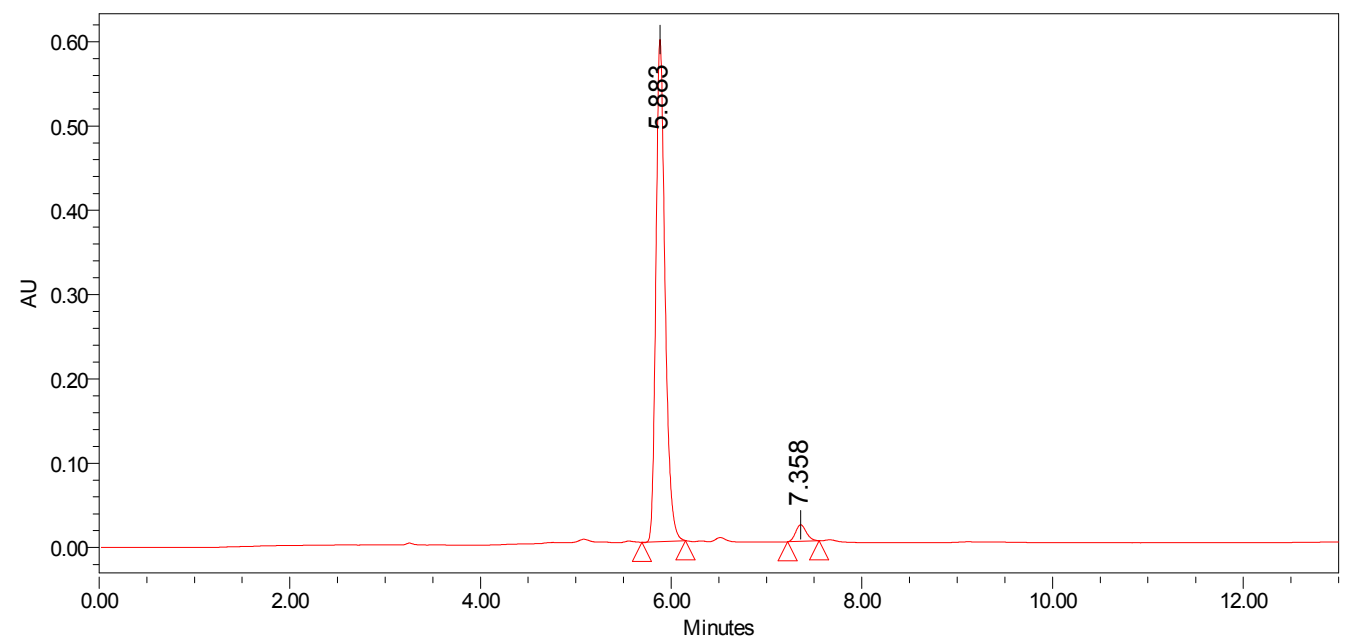

\section{Peak Results}

\begin{tabular}{|c|c|c|c|c|}
\hline & RT & Area & Height & \% Area \\
\hline \hline 1 & 5.883 & 3948375 & 595783 & 96.25 \\
\hline 2 & 7.358 & 153883 & 19668 & 3.75 \\
\hline
\end{tabular}




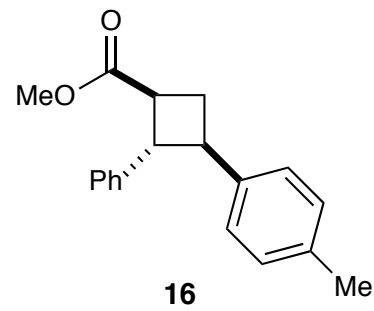

Racemic (Daicel CHIRALCEL ${ }^{\circledR}$ OD-H, 1-40\% IPA:hexanes over $20 \mathrm{~min}, 1 \mathrm{~mL} / \mathrm{min}, 222.0 \mathrm{~nm}$ )

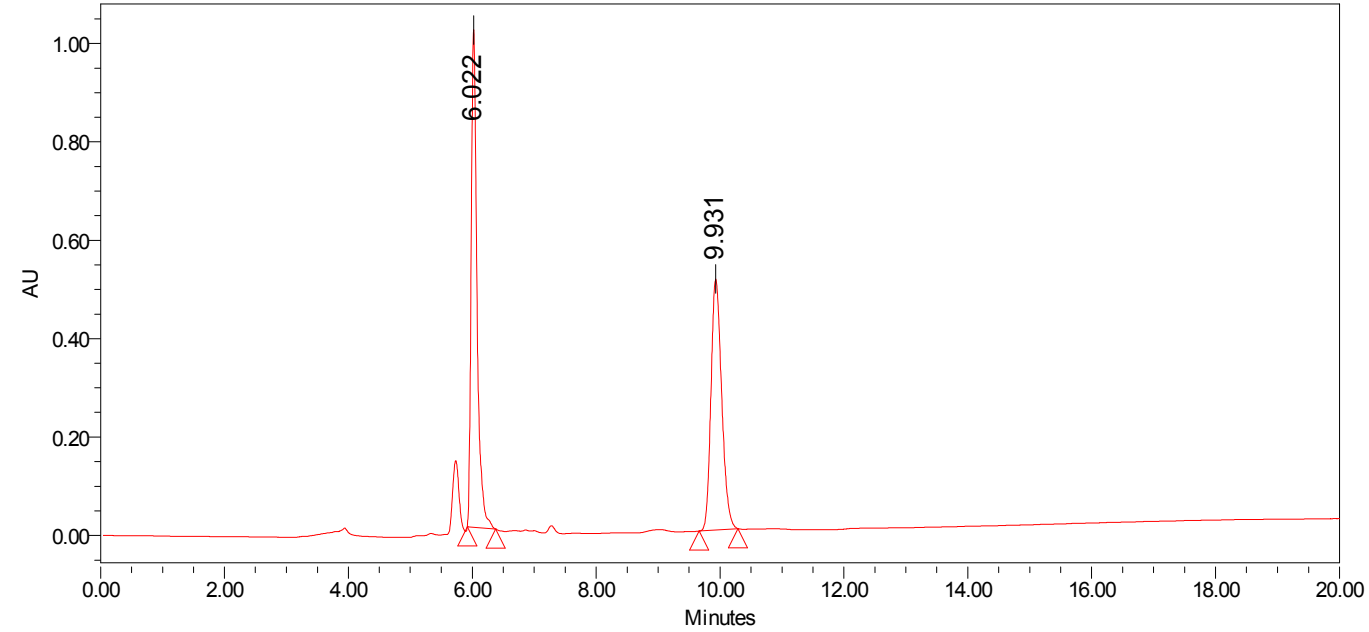

Peak Results

\begin{tabular}{|c|c|c|c|c|}
\hline & RT & Area & Height & \% Area \\
\hline \hline 1 & 6.022 & 6617919 & 1012168 & 51.94 \\
\hline 2 & 9.931 & 6123303 & 510341 & 48.06 \\
\hline
\end{tabular}

\section{Enantioenriched}

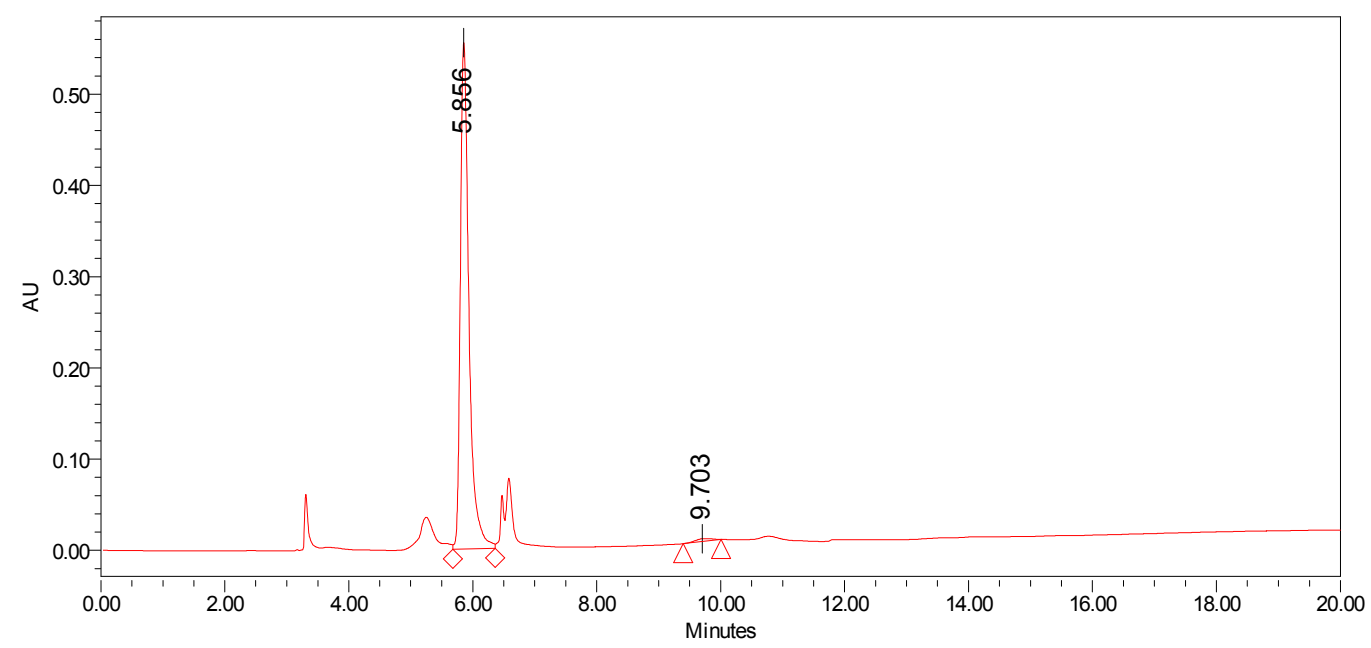

Peak Results

\begin{tabular}{|c|c|c|c|c|}
\hline & RT & Area & Height & \% Area \\
\hline \hline 1 & 5.856 & 5334179 & 555139 & 98.98 \\
\hline 2 & 9.703 & 54952 & 3014 & 1.02 \\
\hline
\end{tabular}




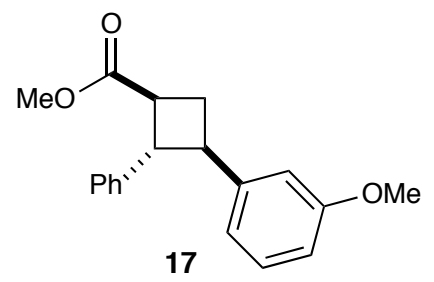

Racemic (Daicel CHIRALCEL ${ }^{\circledR}$ OD-H, 5-50\% IPA:hexanes over $13 \mathrm{~min}, 1 \mathrm{~mL} / \mathrm{min}, 222.0 \mathrm{~nm}$ )

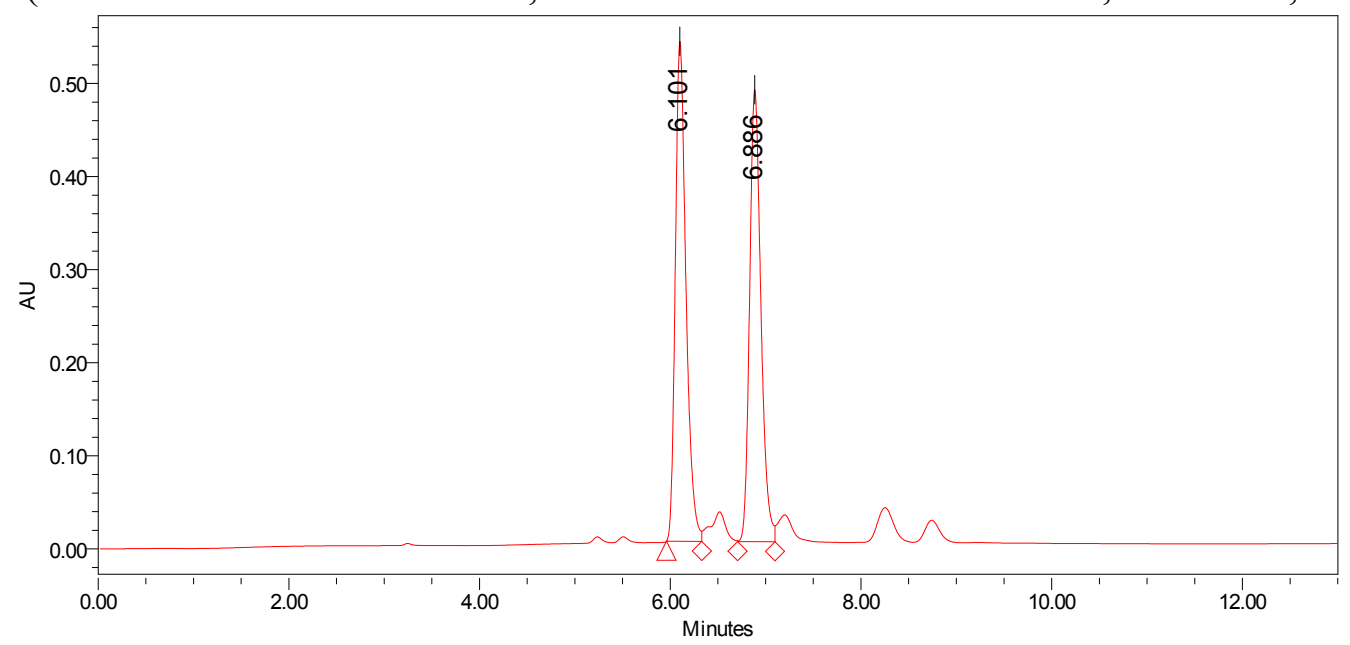

Peak Results

\begin{tabular}{|c|c|c|c|c|}
\hline & RT & Area & Height & \% Area \\
\hline \hline 1 & 6.101 & 4167690 & 537195 & 51.07 \\
\hline 2 & 6.886 & 3993218 & 485683 & 48.93 \\
\hline
\end{tabular}

Enantioenriched

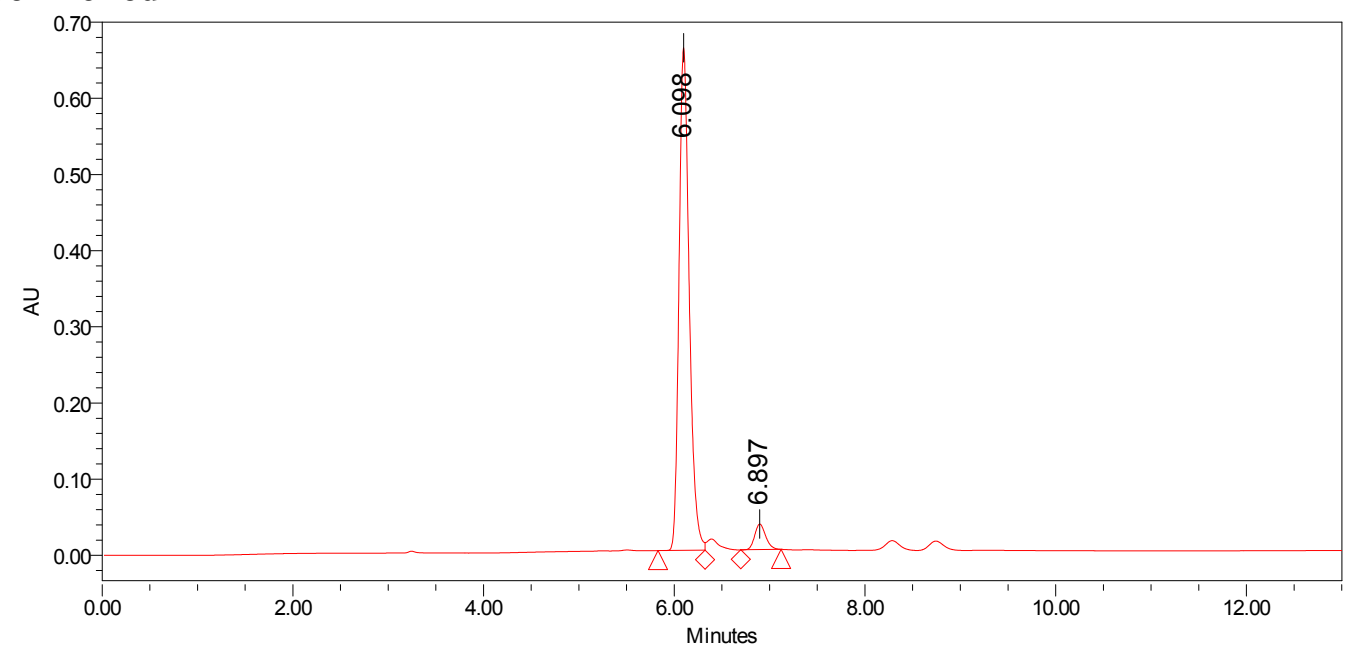

Peak Results

\begin{tabular}{|c|c|c|c|c|}
\hline & RT & Area & Height & \% Area \\
\hline \hline 1 & 6.098 & 4895403 & 659959 & 94.82 \\
\hline 2 & 6.897 & 267668 & 33513 & 5.18 \\
\hline
\end{tabular}




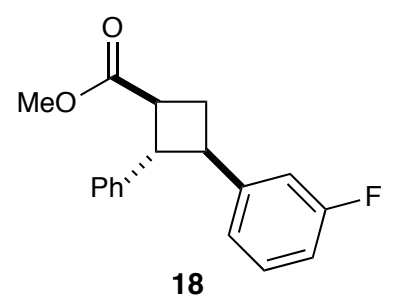

Racemic (Daicel CHIRALCEL ${ }^{\circledR}$ IC, 1-40\% IPA:hexanes over $20 \mathrm{~min}, 1 \mathrm{~mL} / \mathrm{min}, 222.0 \mathrm{~nm}$ )

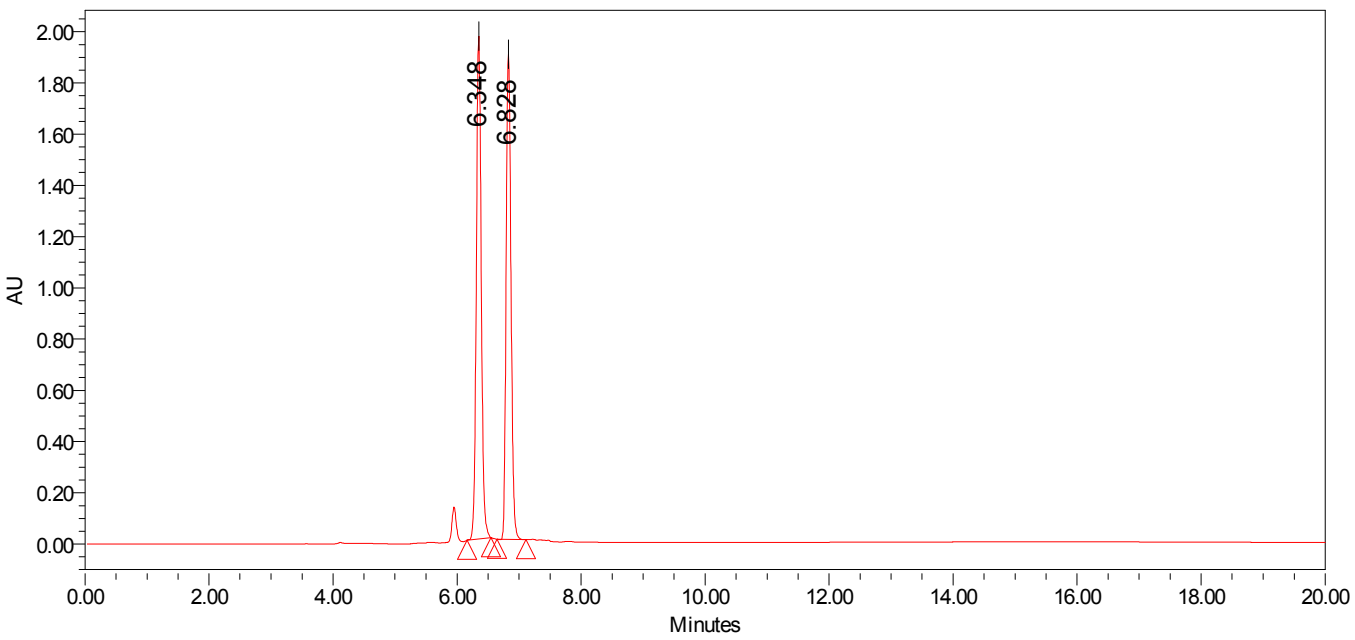

\section{Peak Results}

\begin{tabular}{|c|c|c|c|c|}
\hline & RT & Area & Height & \% Area \\
\hline \hline 1 & 6.348 & 10871511 & 1963443 & 51.22 \\
\hline 2 & 6.828 & 10352934 & 1893978 & 48.78 \\
\hline
\end{tabular}

\section{Enantioenriched}

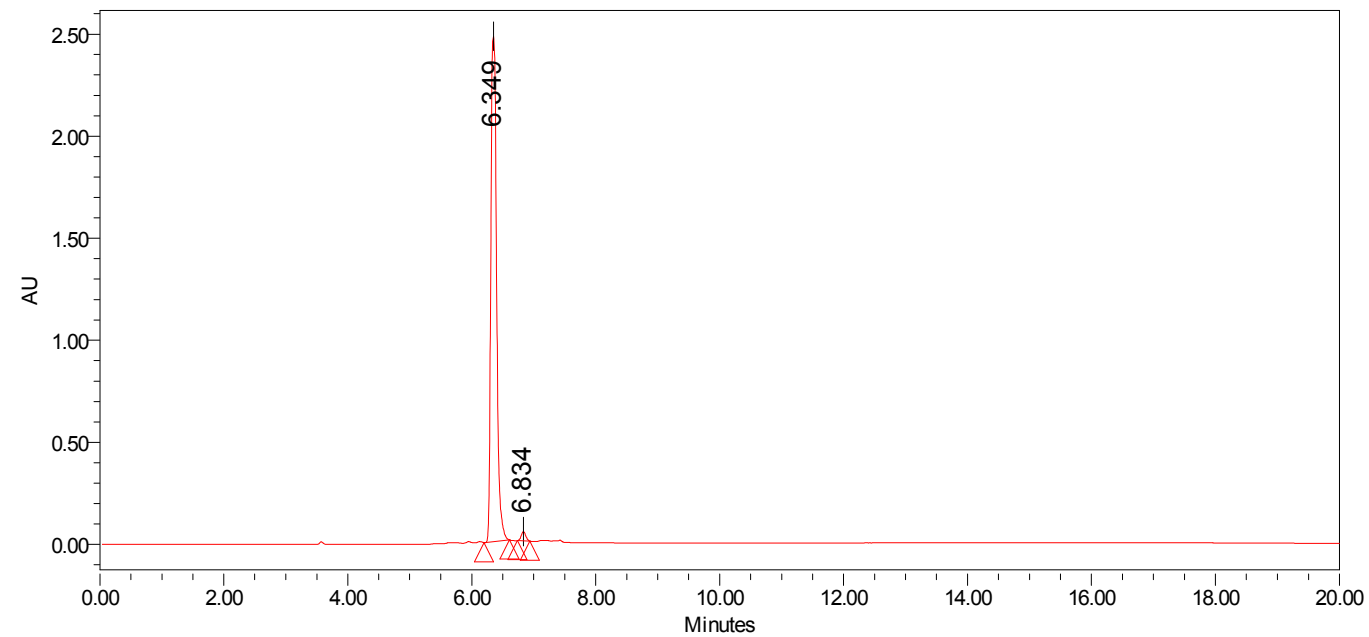

\section{Peak Results}

\begin{tabular}{|c|c|c|c|c|}
\hline & RT & Area & Height & \% Area \\
\hline \hline 1 & 6.349 & 15973477 & 2477308 & 98.57 \\
\hline 2 & 6.834 & 231303 & 45513 & 1.43 \\
\hline
\end{tabular}




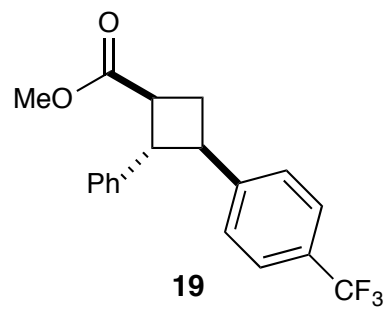

Racemic (Daicel CHIRALCEL ${ }^{\circledR}$ OD-H, 5-50\% IPA:hexanes over $13 \mathrm{~min}, 1 \mathrm{~mL} / \mathrm{min}, 222.0 \mathrm{~nm}$ )

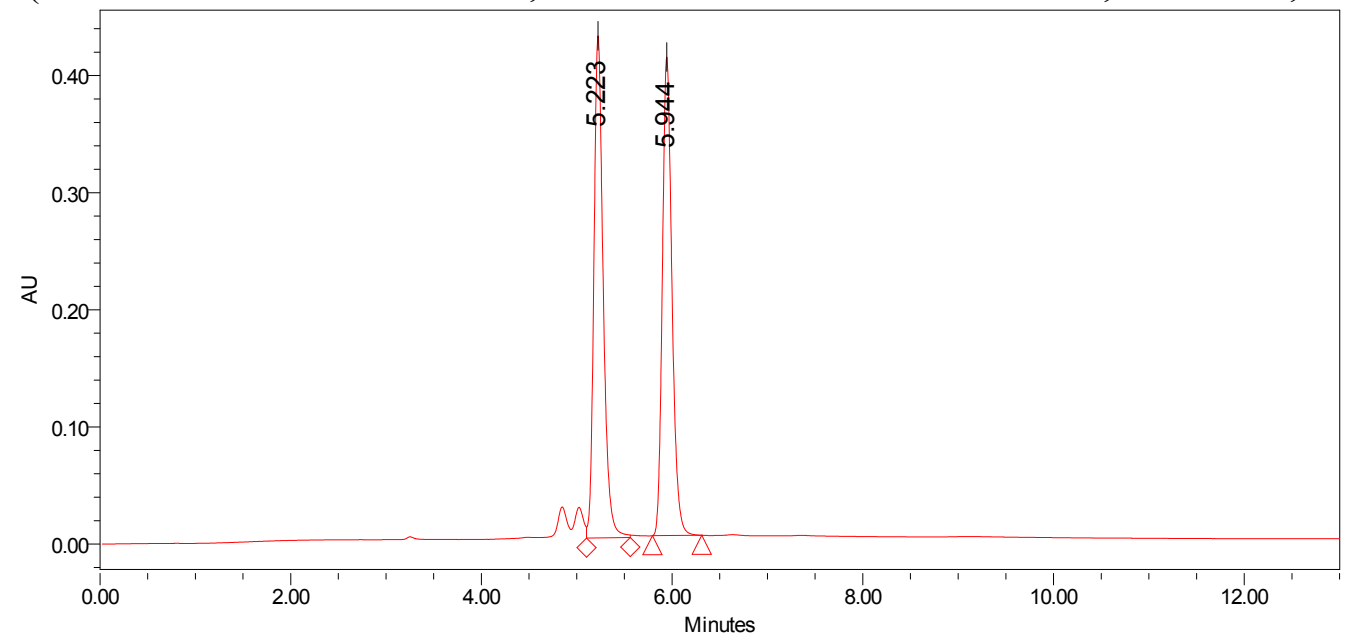

Peak Results

\begin{tabular}{|c|c|c|c|c|}
\hline & RT & Area & Height & \% Area \\
\hline \hline 1 & 5.223 & 2908875 & 428731 & 50.53 \\
\hline 2 & 5.944 & 2848215 & 408653 & 49.47 \\
\hline
\end{tabular}

\section{Enantioenriched}

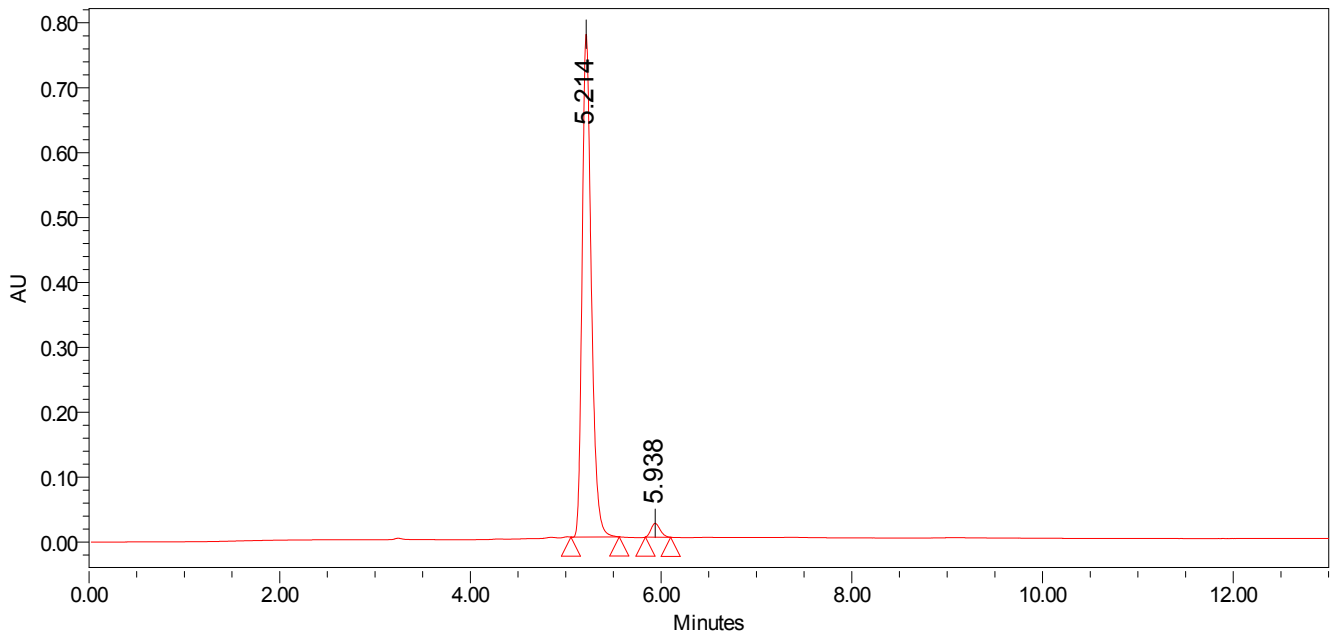

Peak Results

\begin{tabular}{|c|c|c|c|c|}
\hline & RT & Area & Height & \% Area \\
\hline \hline 1 & 5.214 & 5162770 & 774821 & 97.36 \\
\hline 2 & 5.938 & 139916 & 21261 & 2.64 \\
\hline
\end{tabular}




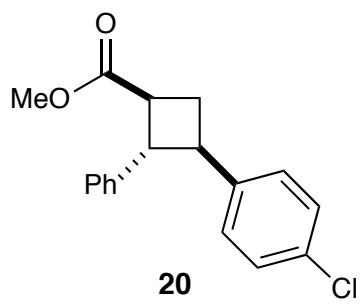

Racemic (Daicel CHIRALCEL ${ }^{\circledR}$ OD-H, 1-40\% IPA:hexanes over $20 \mathrm{~min}, 1 \mathrm{~mL} / \mathrm{min}, 222.0 \mathrm{~nm}$ )

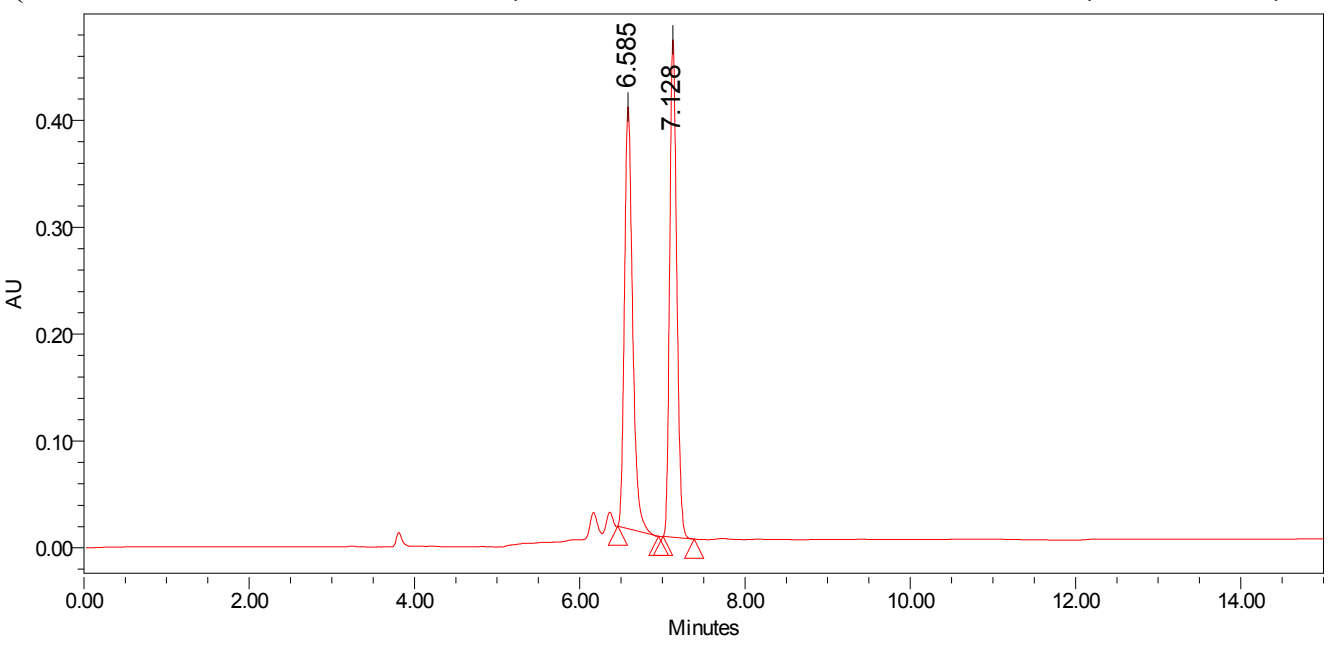

\section{Peak Results}

\begin{tabular}{|c|c|c|c|c|}
\hline & RT & Area & Height & \% Area \\
\hline \hline 1 & 6.585 & 2720082 & 394636 & 49.60 \\
\hline 2 & 7.128 & 2763822 & 465774 & 50.40 \\
\hline
\end{tabular}

\section{Enantioenriched}

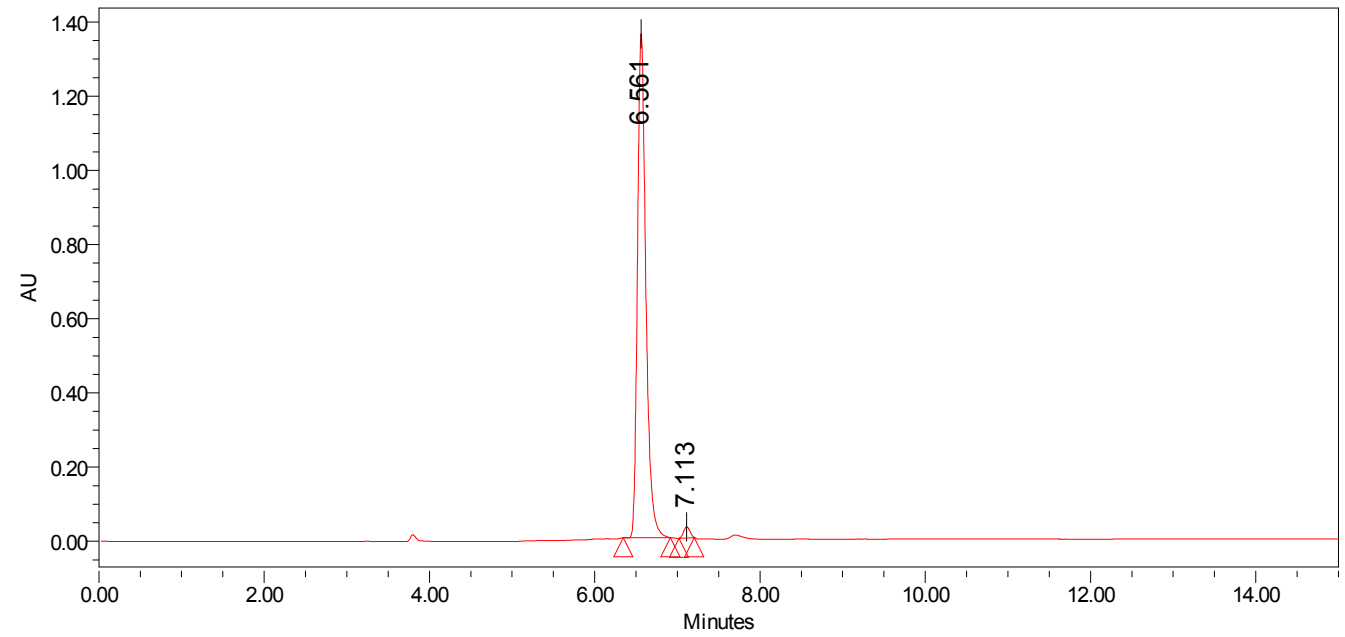

Peak Results

\begin{tabular}{|c|c|c|c|c|}
\hline & RT & Area & Height & \% Area \\
\hline 1 & 6.561 & 9596463 & 1359393 & 98.35 \\
\hline 2 & 7.113 & 161309 & 30253 & 1.65 \\
\hline
\end{tabular}




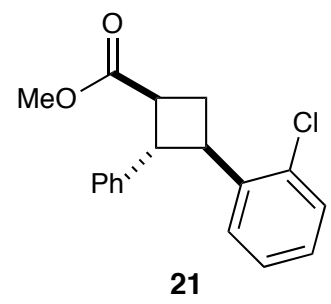

Racemic (Daicel CHIRALCEL ${ }^{\circledR}$ OD-H, 5-50\% IPA:hexanes over $13 \mathrm{~min}, 1 \mathrm{~mL} / \mathrm{min}, 222.0 \mathrm{~nm}$ )

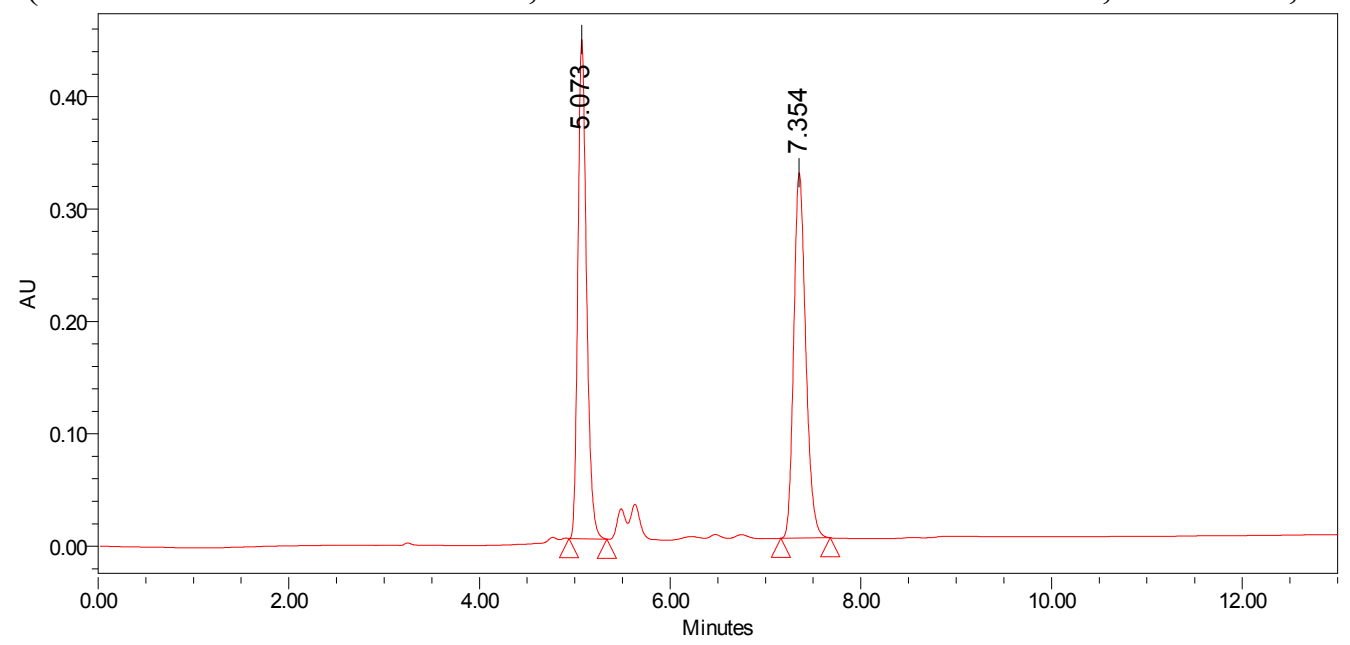

Peak Results

\begin{tabular}{|c|c|c|c|c|}
\hline & RT & Area & Height & \% Area \\
\hline \hline 1 & 5.073 & 2788651 & 444284 & 49.60 \\
\hline 2 & 7.354 & 2834015 & 325080 & 50.40 \\
\hline
\end{tabular}

\section{Enantioenriched}

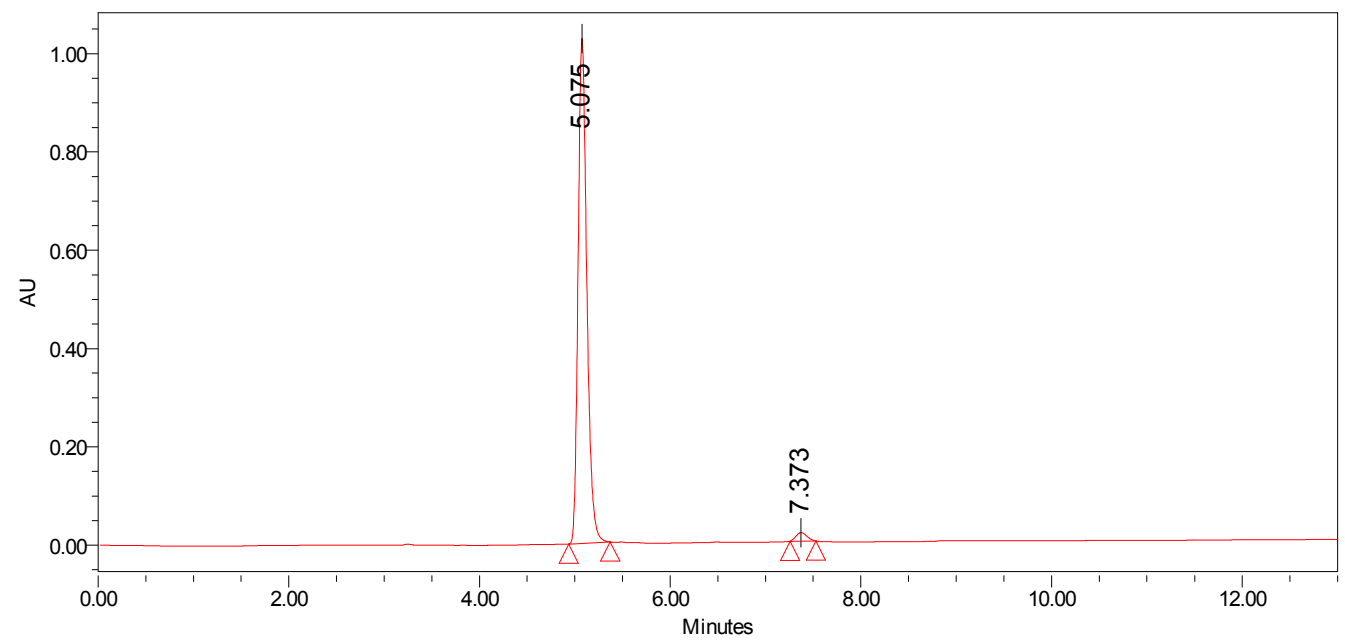

Peak Results

\begin{tabular}{|c|c|c|c|c|}
\hline & RT & Area & Height & \% Area \\
\hline \hline 1 & 5.075 & 6538986 & 1027820 & 97.97 \\
\hline 2 & 7.373 & 135710 & 17242 & 2.03 \\
\hline
\end{tabular}




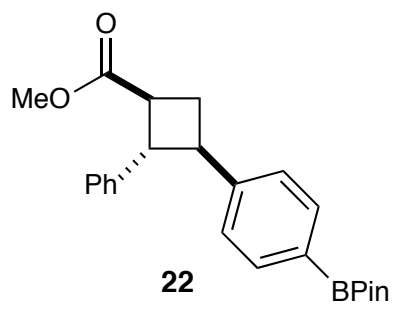

Racemic (Daicel CHIRALCEL ${ }^{\circledR}$ IC, 1-40\% IPA:hexanes over $20 \mathrm{~min}, 1 \mathrm{~mL} / \mathrm{min}, 222.0 \mathrm{~nm}$ )

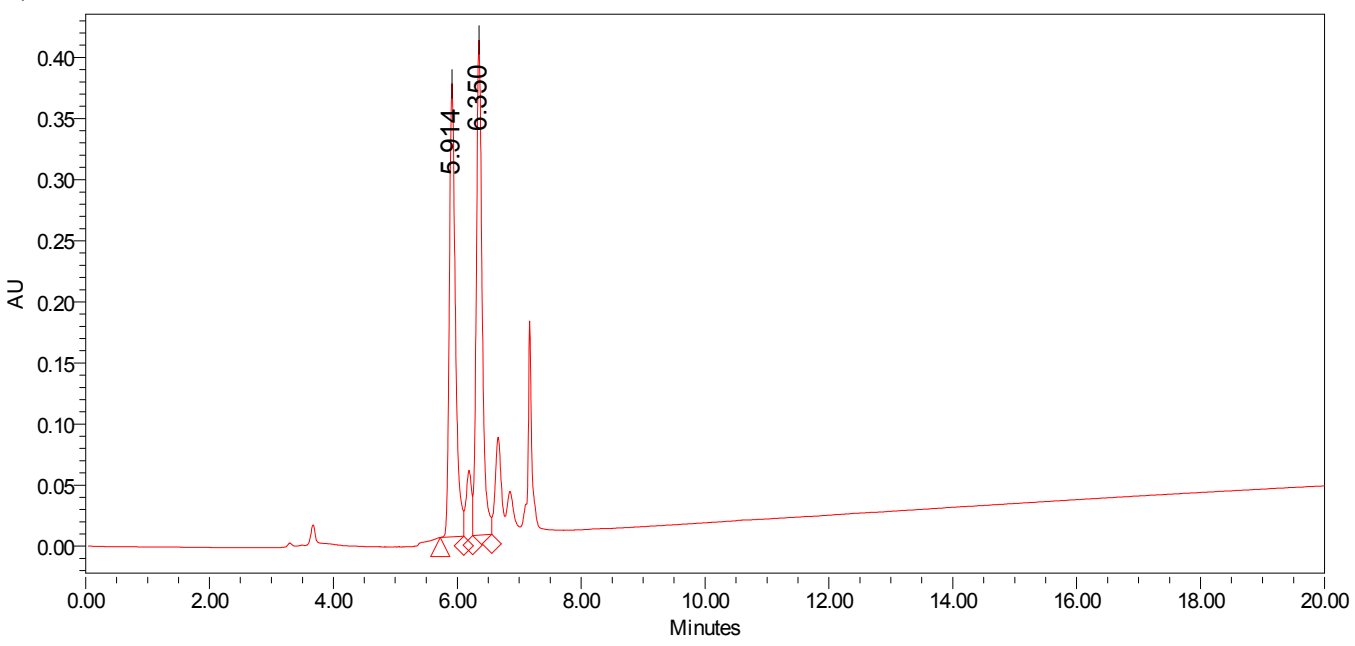

Peak Results

\begin{tabular}{|c|c|c|c|c|}
\hline & RT & Area & Height & \% Area \\
\hline 1 & 5.914 & 2519166 & 370995 & 48.94 \\
\hline 2 & 6.350 & 2628640 & 405362 & 51.06 \\
\hline
\end{tabular}

\section{Enantioenriched}

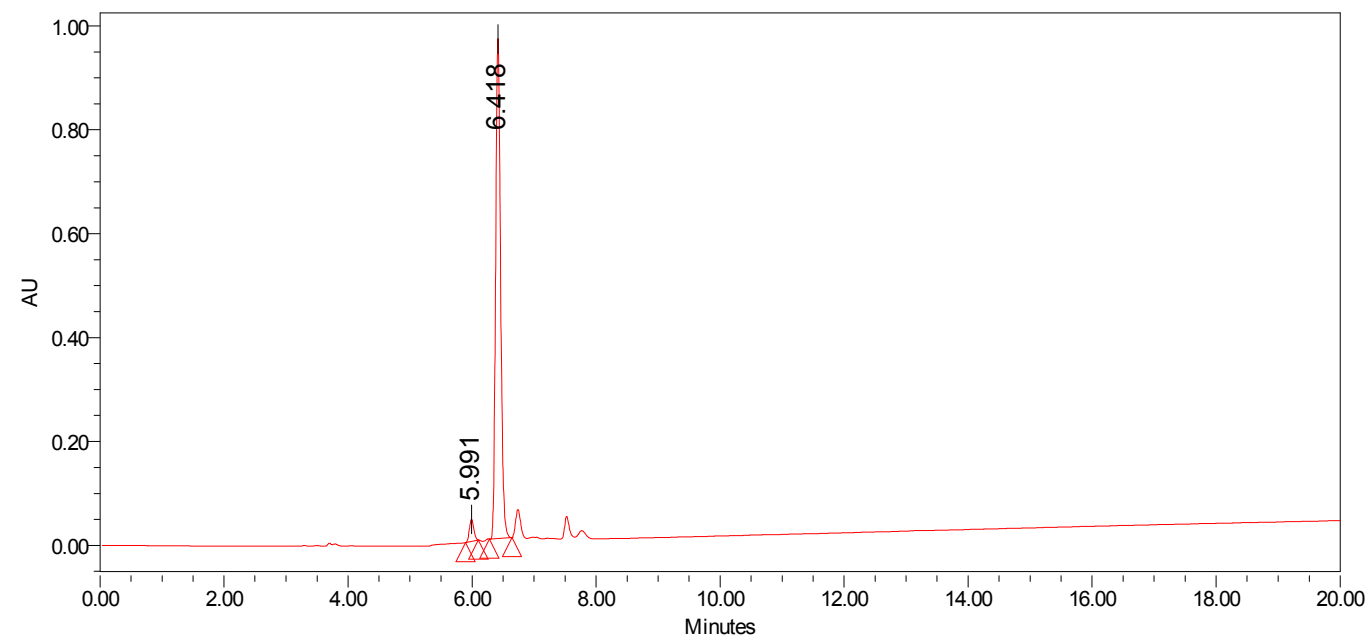

Peak Results

\begin{tabular}{|c|c|c|c|c|}
\hline & RT & Area & Height & \% Area \\
\hline 1 & 5.991 & 212213 & 42518 & 3.81 \\
\hline 2 & 6.418 & 5351911 & 962642 & 96.19 \\
\hline
\end{tabular}




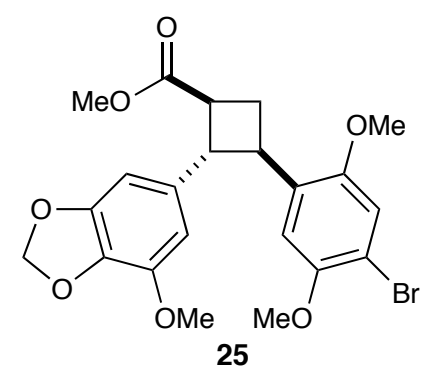

Racemic (Daicel CHIRALPAK ${ }^{\circledR}$ OD-H, 5-50\% IPA:hexanes over $13 \mathrm{~min}, 1 \mathrm{~mL} / \mathrm{min}, 222.0 \mathrm{~nm}$ )

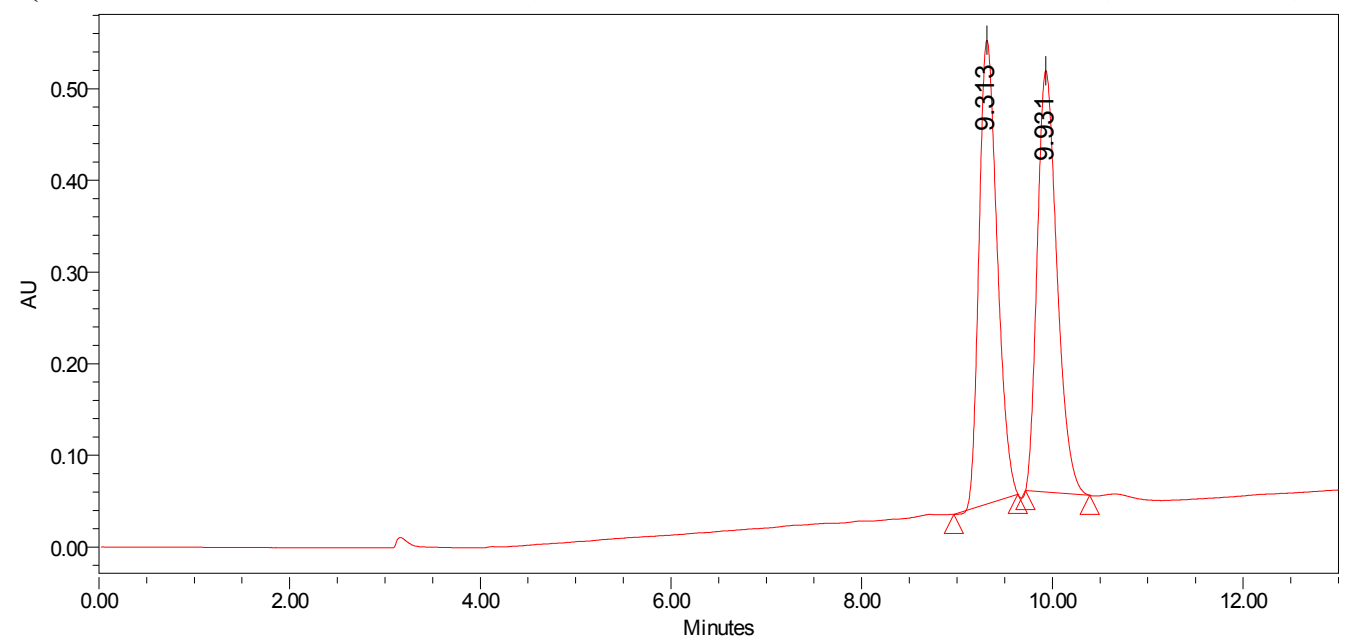

Peak Results

\begin{tabular}{|c|c|c|c|c|}
\hline & RT & Area & Height & \% Area \\
\hline 1 & 9.313 & 6713611 & 506088 & 50.36 \\
\hline 2 & 9.931 & 6617844 & 459606 & 49.64 \\
\hline
\end{tabular}

\section{Enantioenriched}

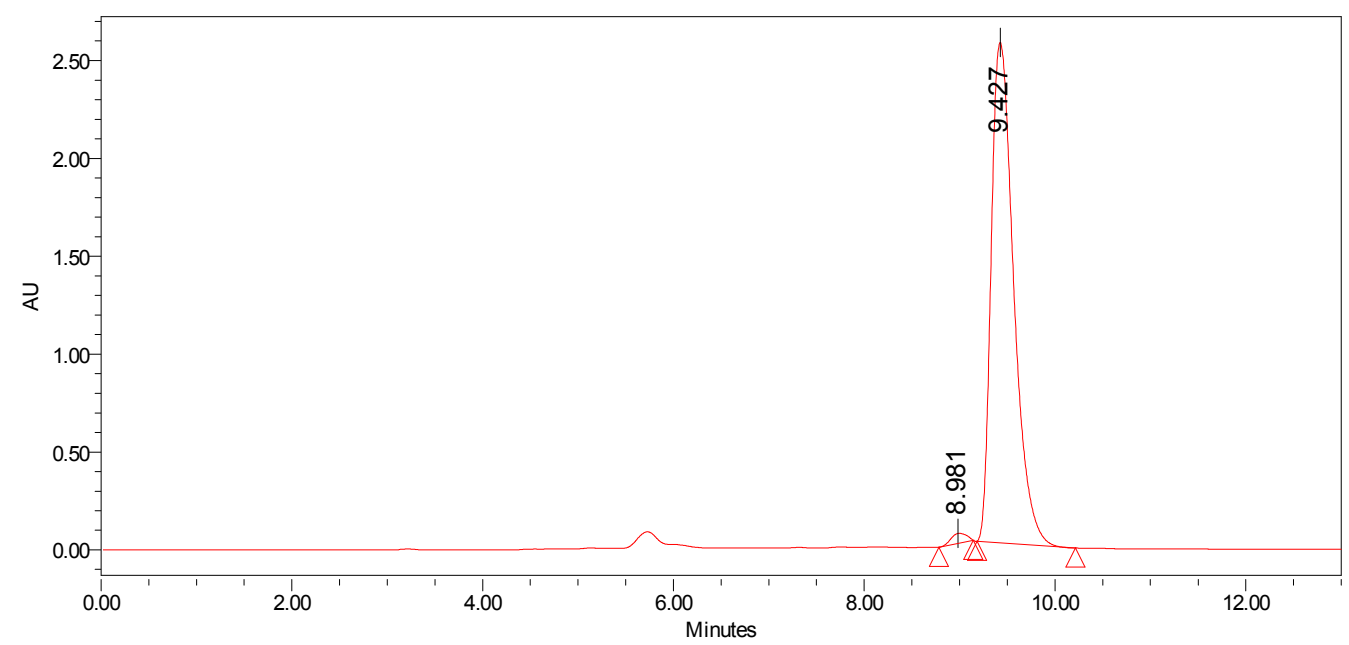

Peak Results

\begin{tabular}{|c|c|c|c|c|}
\hline & RT & Area & Height & \% Area \\
\hline \hline 1 & 8.981 & 556397 & 50487 & 1.31 \\
\hline 2 & 9.427 & 41866658 & 2558349 & 98.69 \\
\hline
\end{tabular}




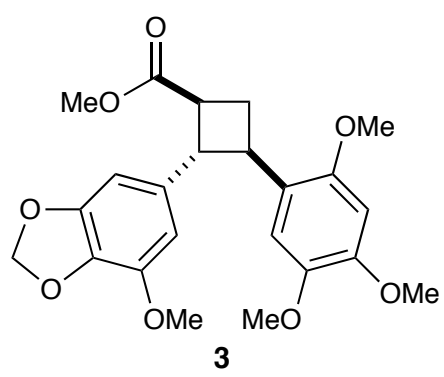

Racemic (Daicel CHIRALPAK ${ }^{\circledR}$ OJ-H, 40\% EtOH:hexanes over $40 \mathrm{~min}, 4 \mathrm{~mL} / \mathrm{min}, 222.0 \mathrm{~nm}$ )

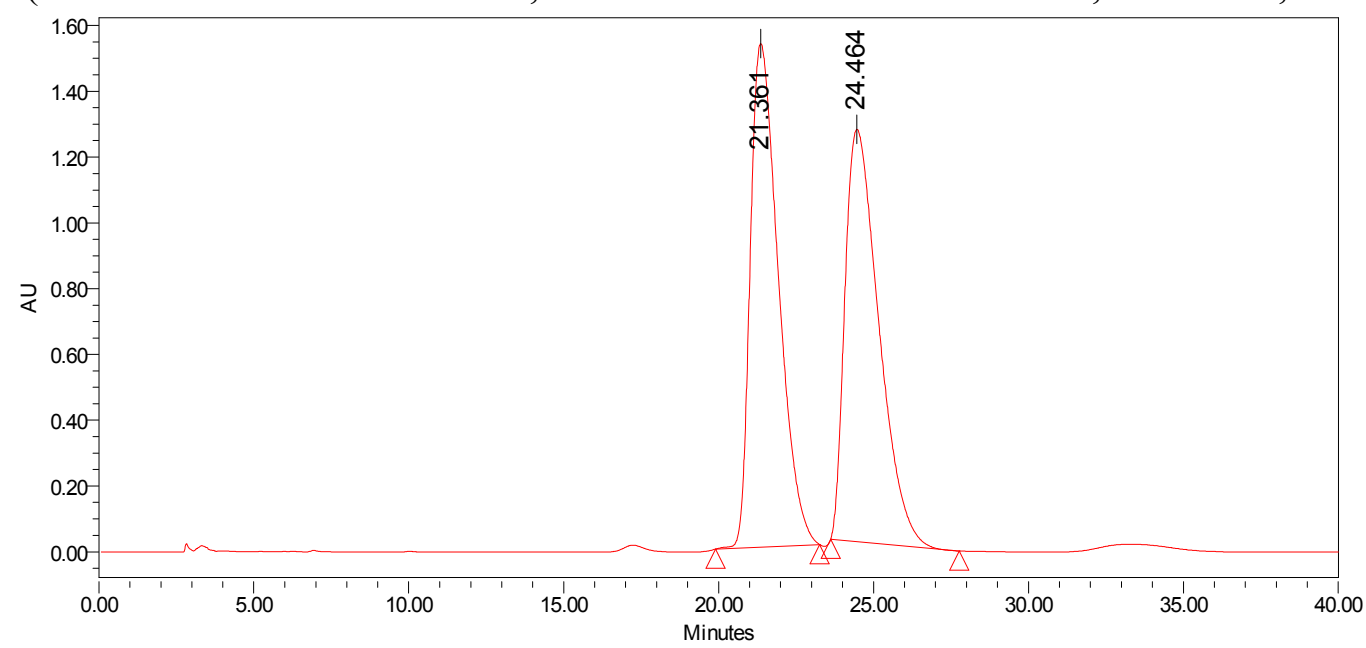

Peak Results

\begin{tabular}{|c|c|c|c|c|}
\hline & RT & Area & Height & \% Area \\
\hline \hline 1 & 21.361 & 96695479 & 1531585 & 50.39 \\
\hline 2 & 24.464 & 95206991 & 1253920 & 49.61 \\
\hline
\end{tabular}

\section{Enantioenriched}

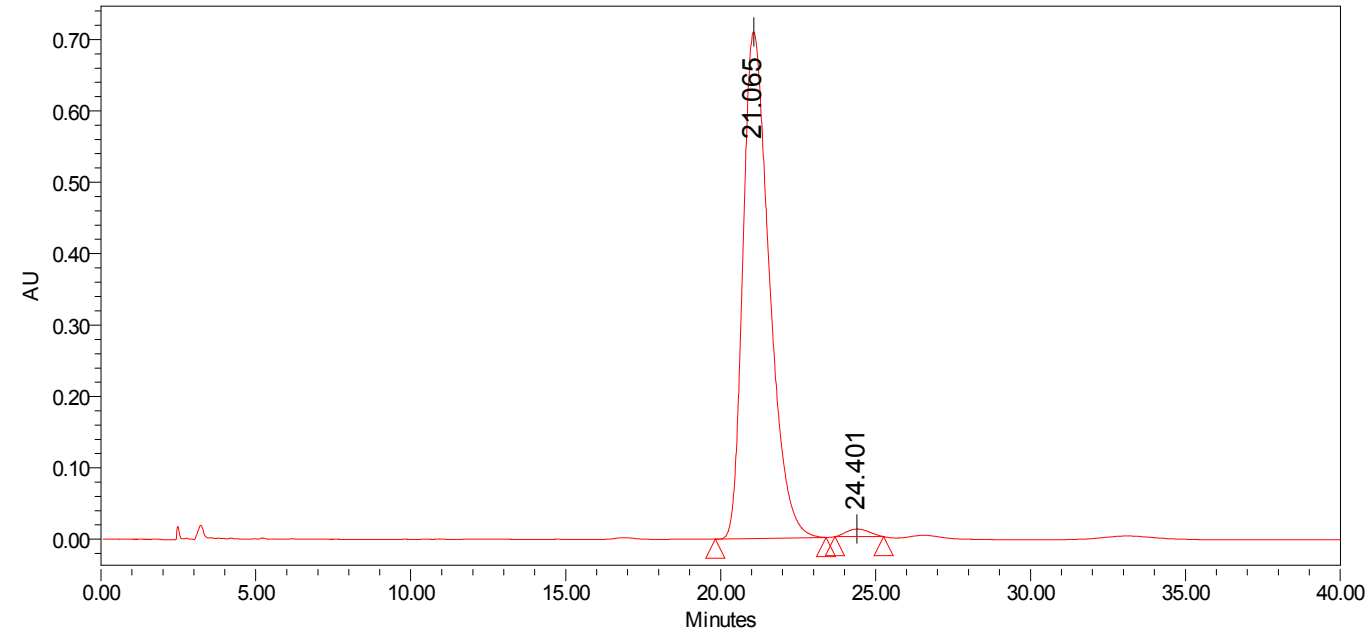

Peak Results

\begin{tabular}{|c|c|c|c|c|}
\hline & RT & Area & Height & \% Area \\
\hline 1 & 21.065 & 41785892 & 709963 & 98.69 \\
\hline 2 & 24.401 & 553368 & 10701 & 1.31 \\
\hline
\end{tabular}




\section{NMR Spectra}

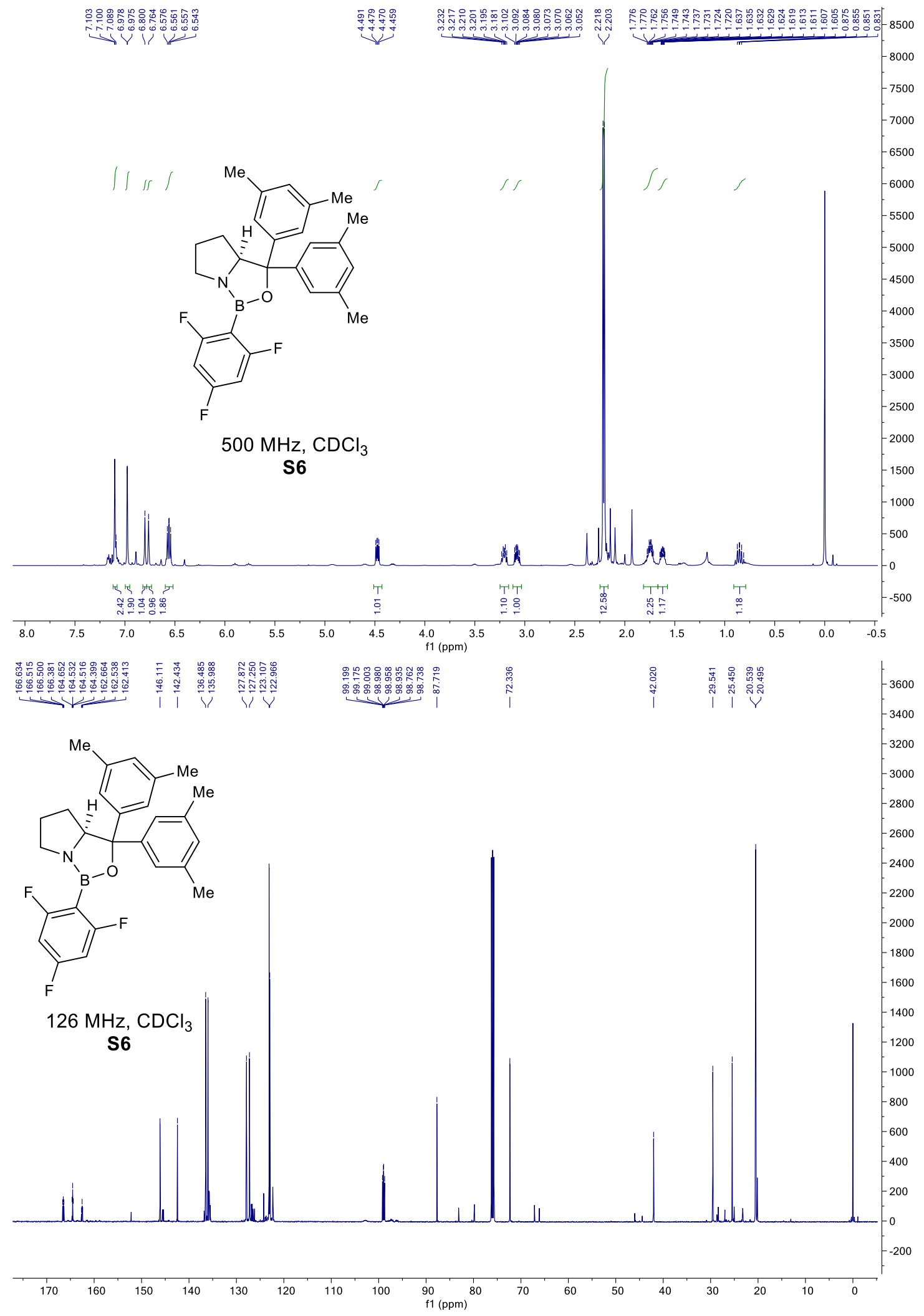




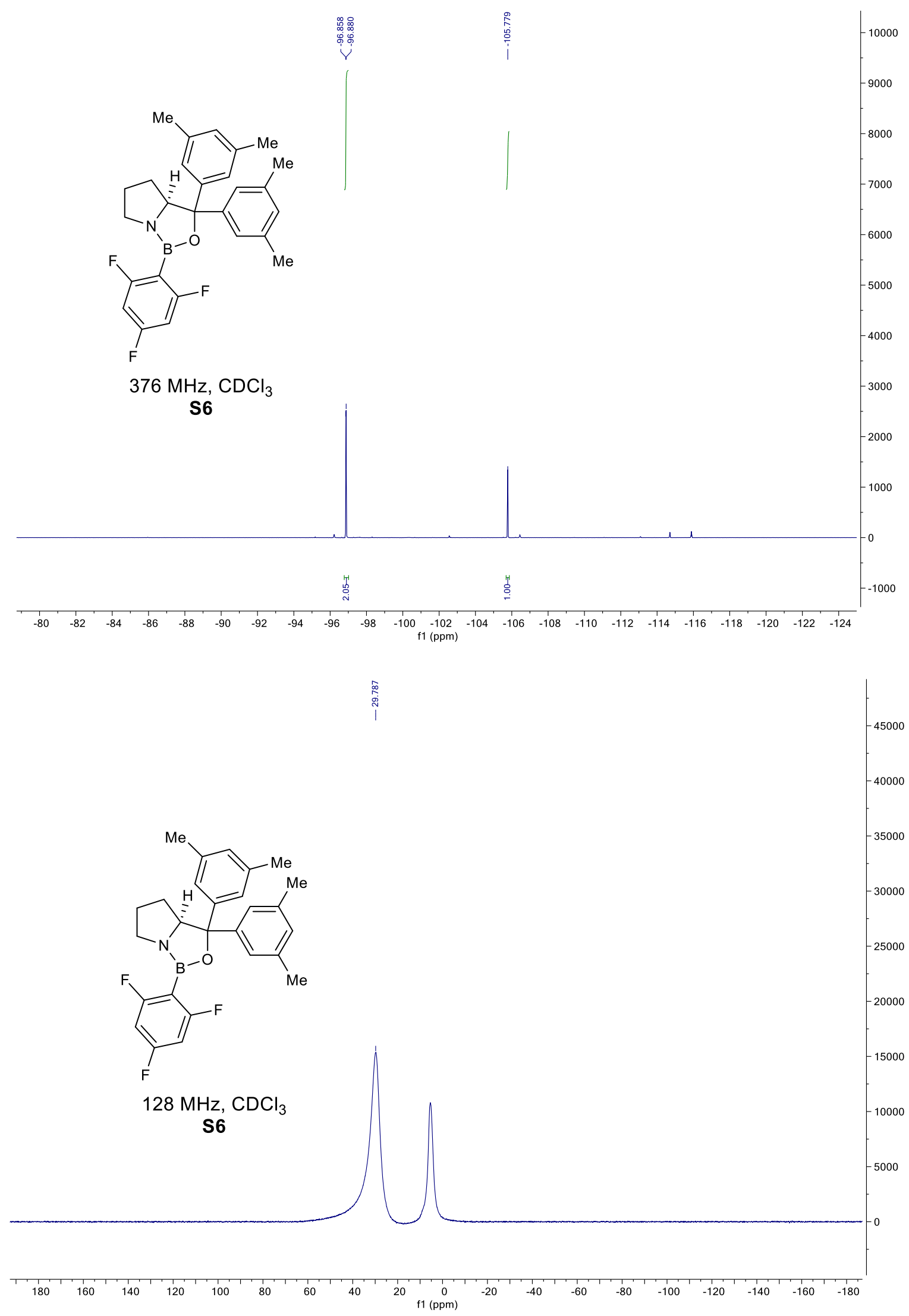




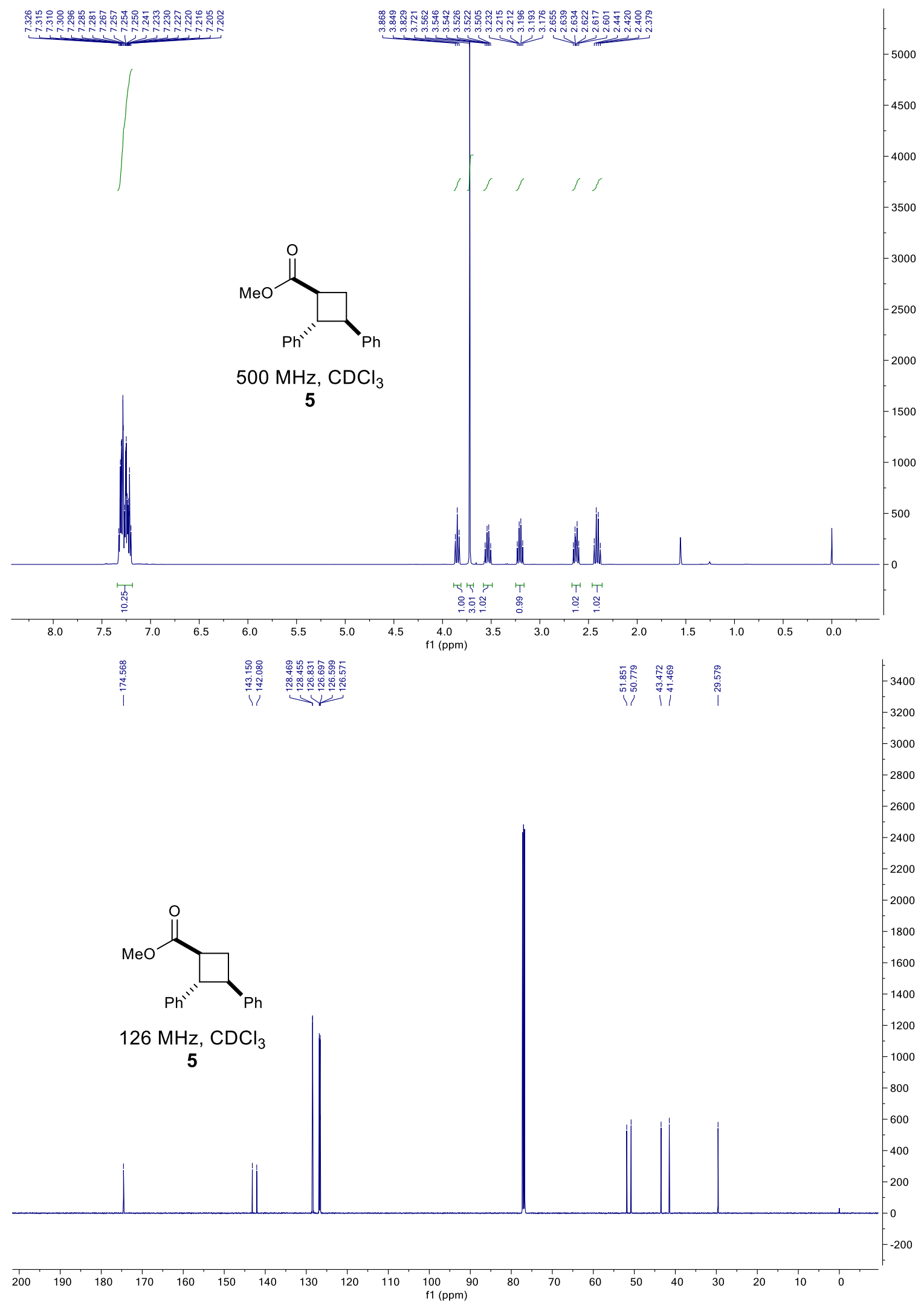




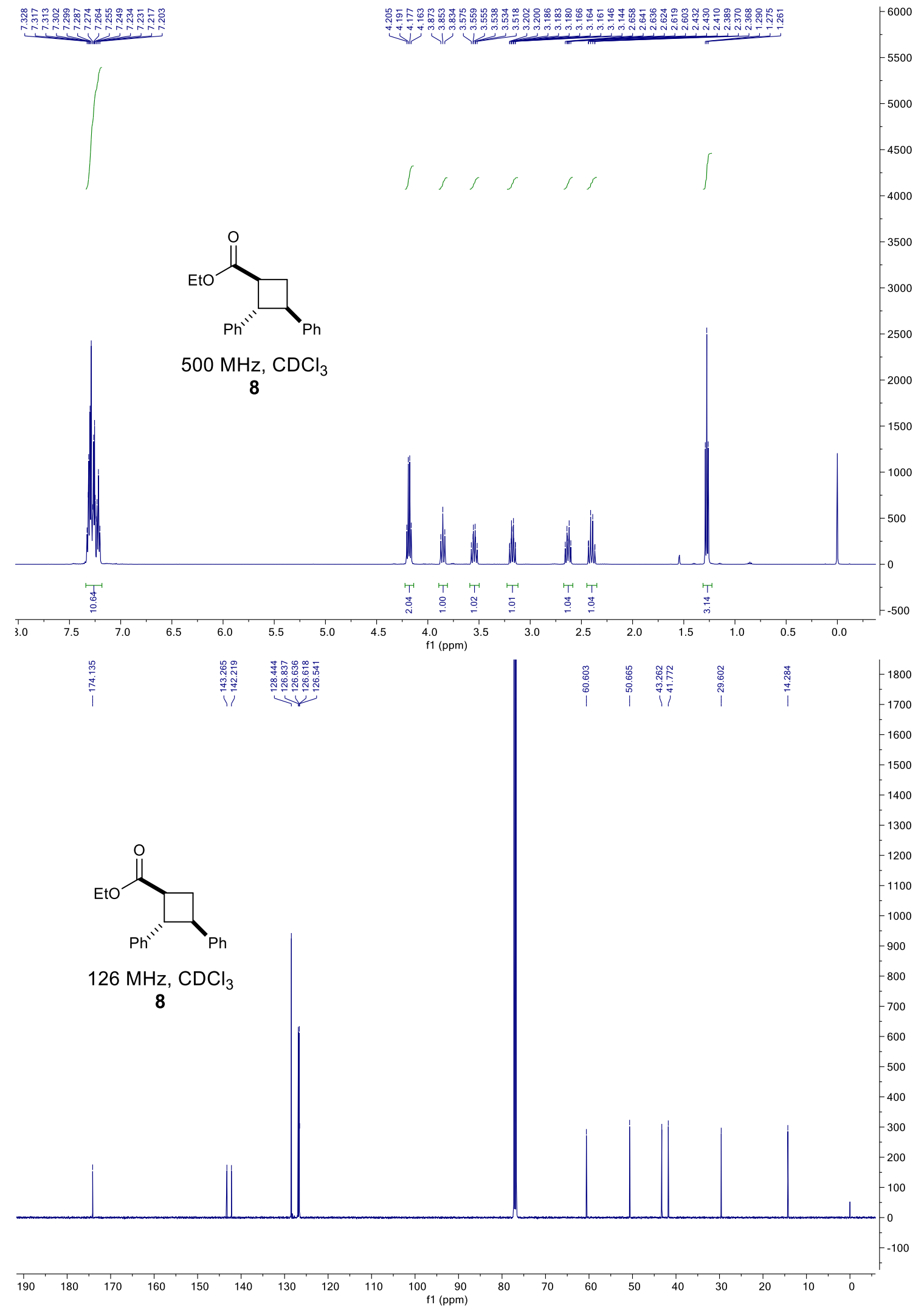




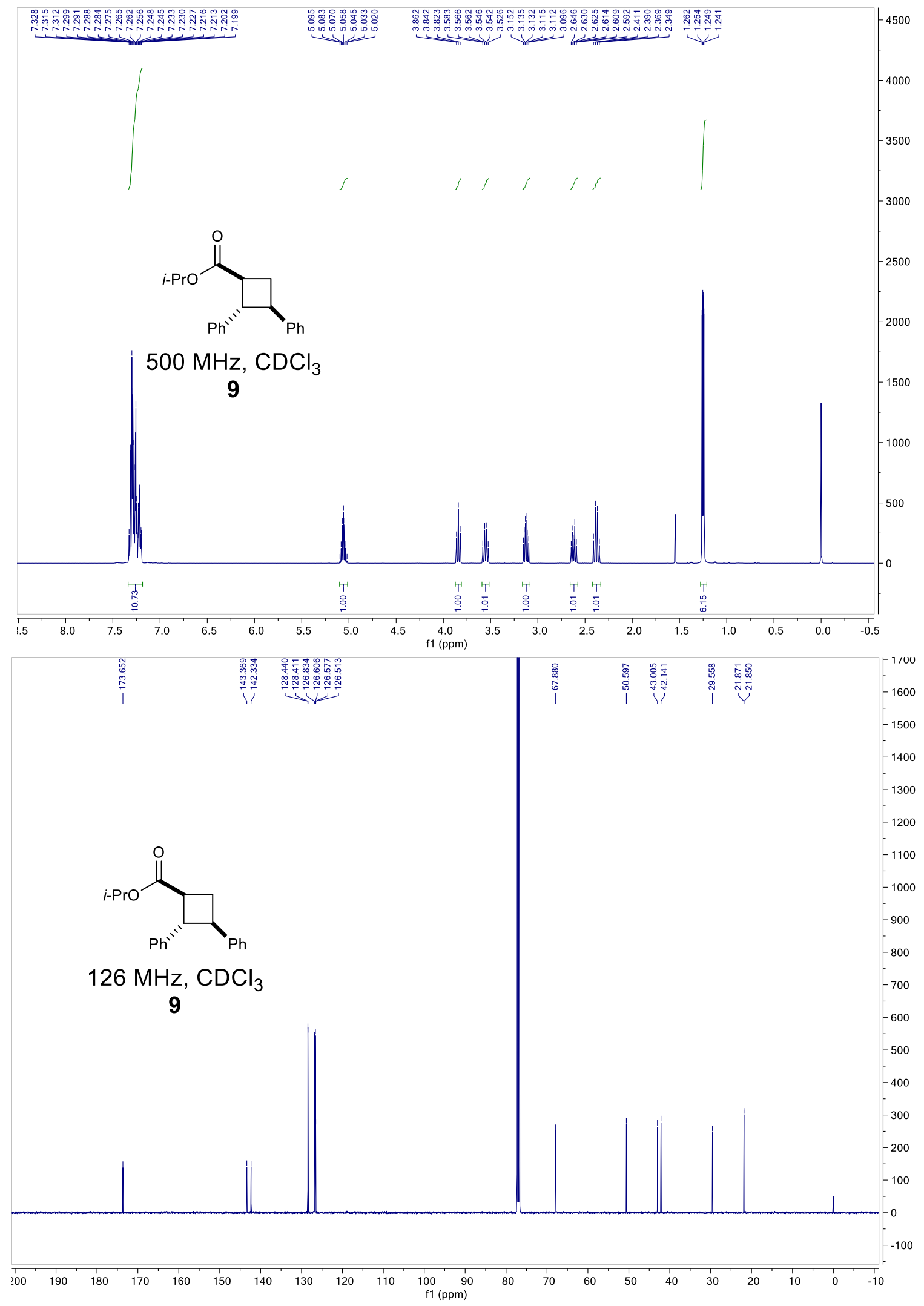




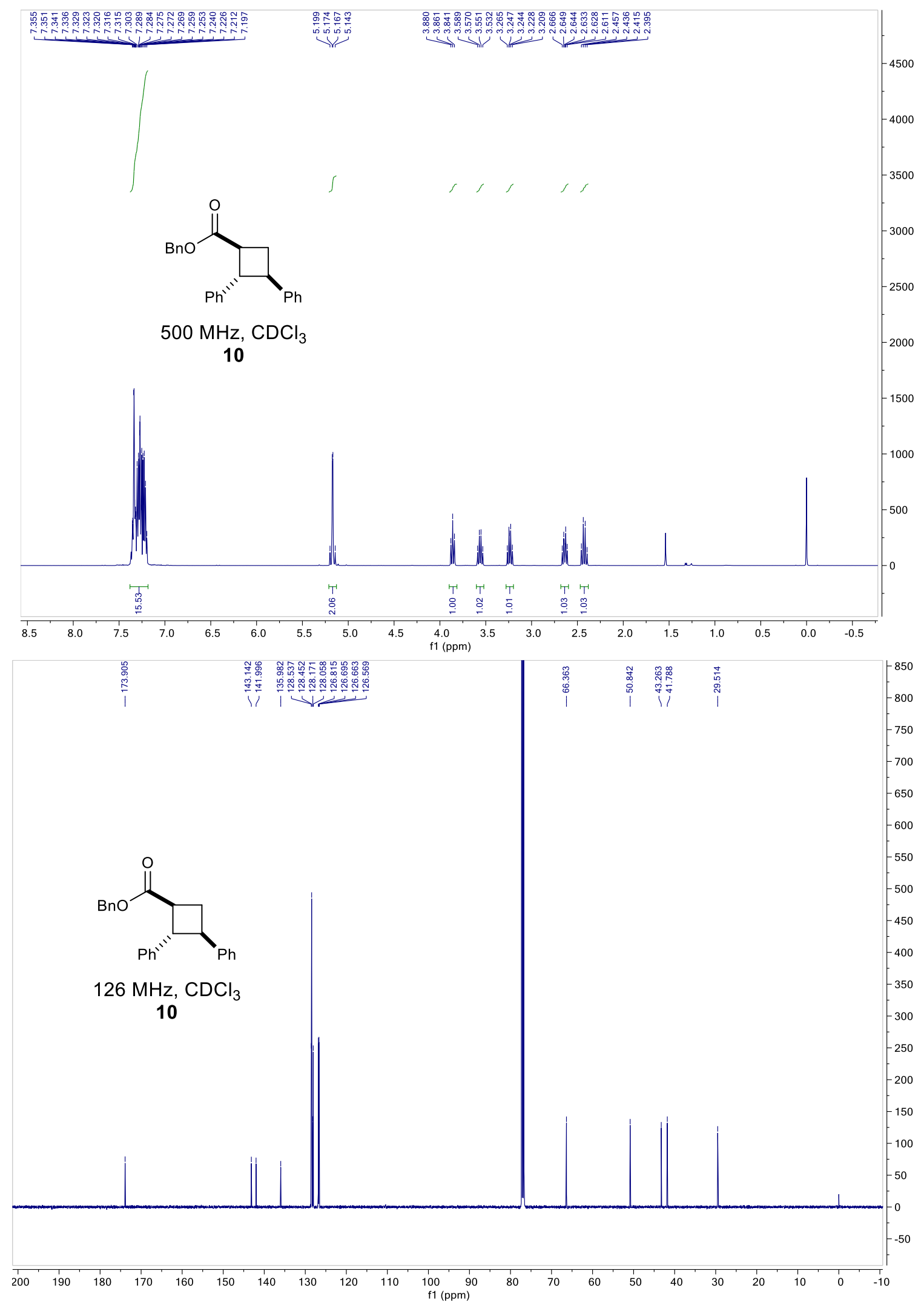




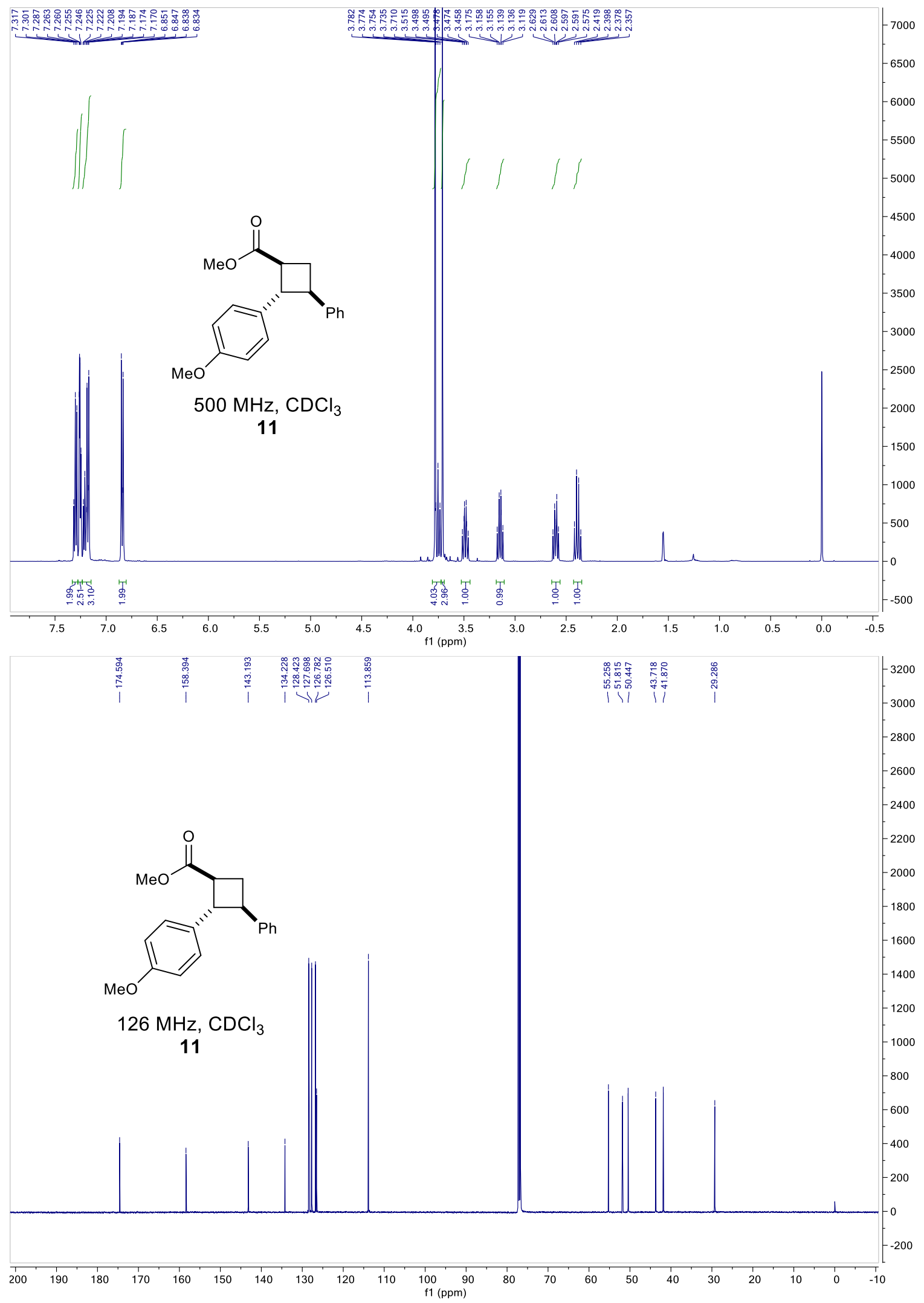




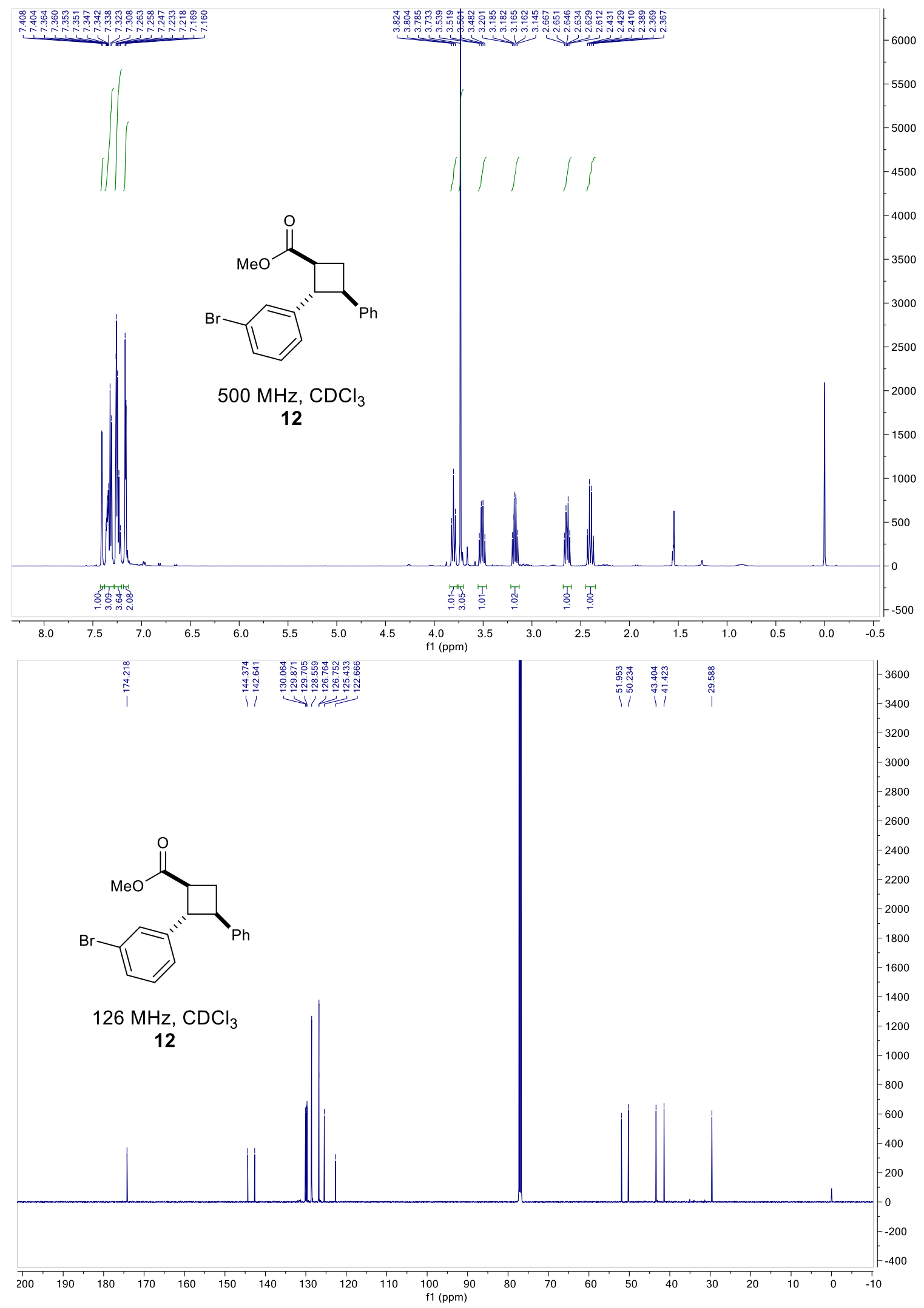




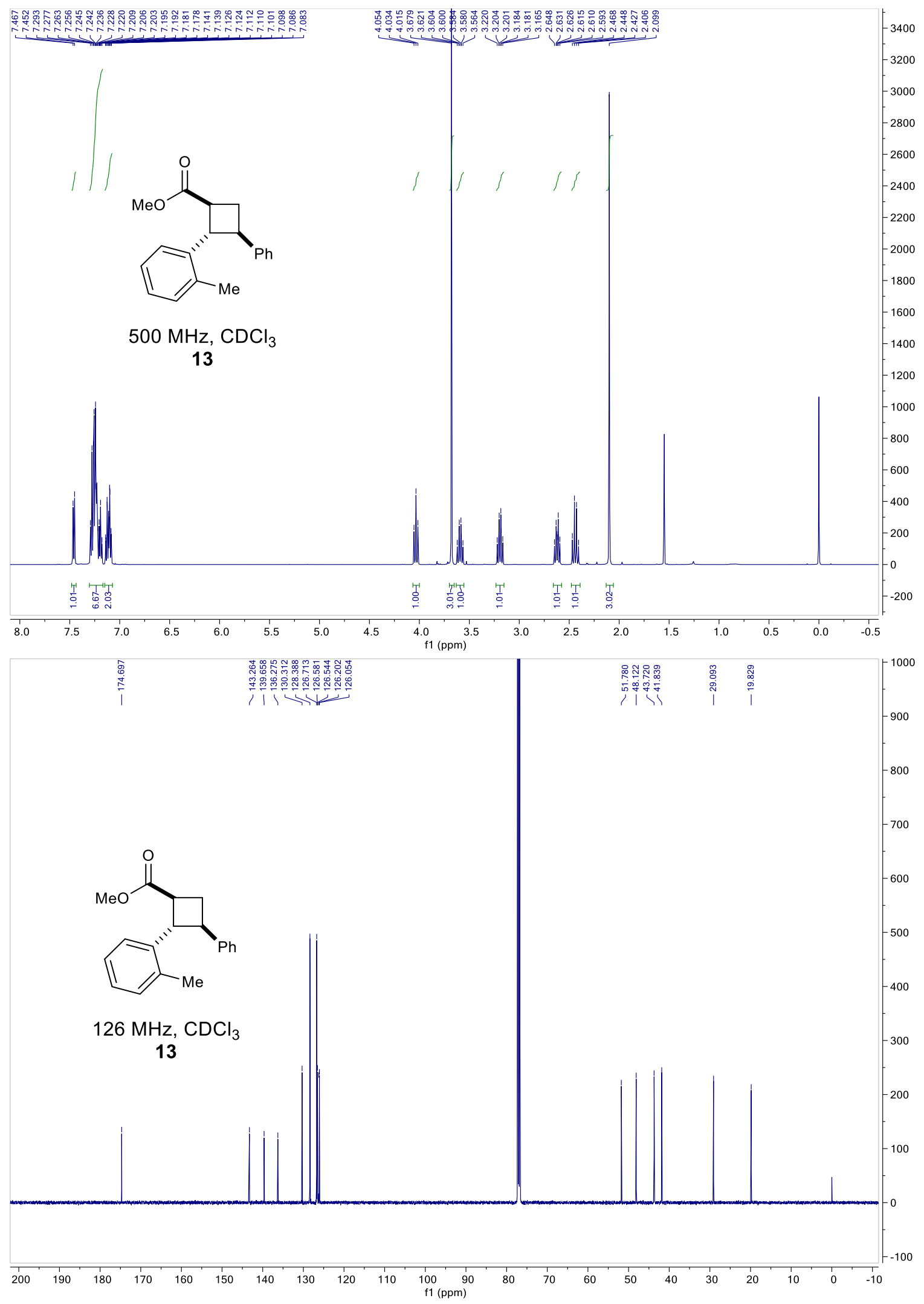




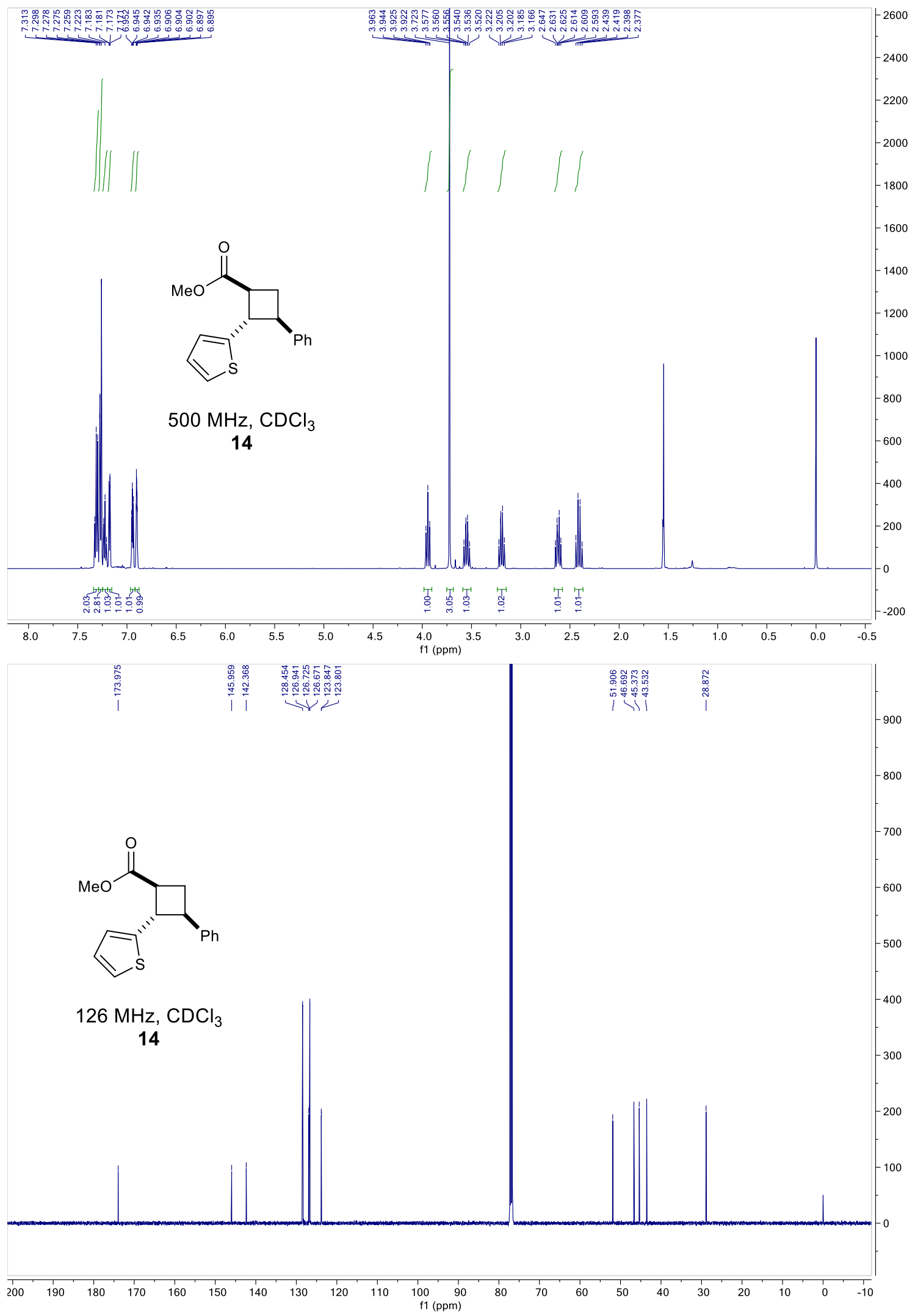




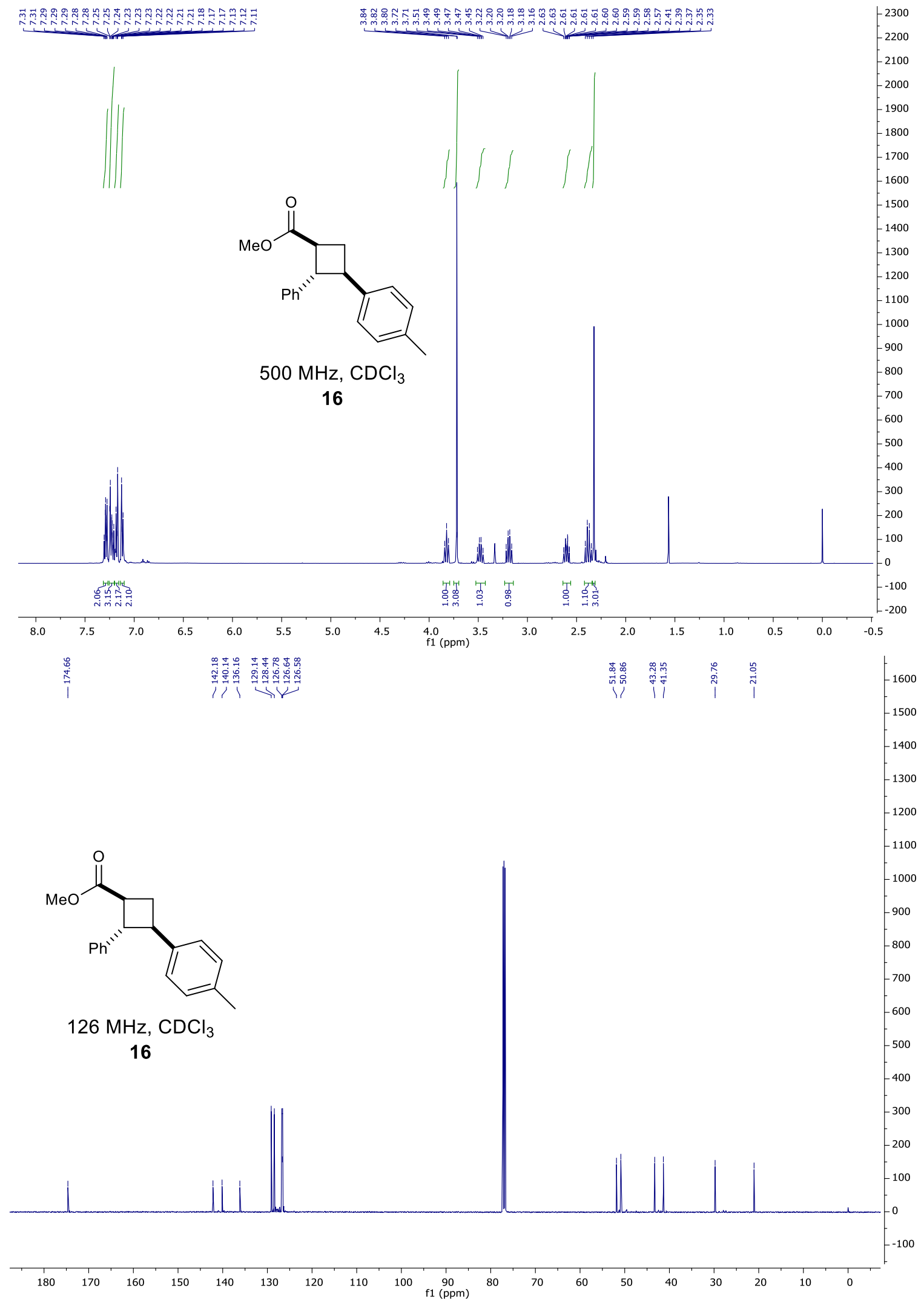




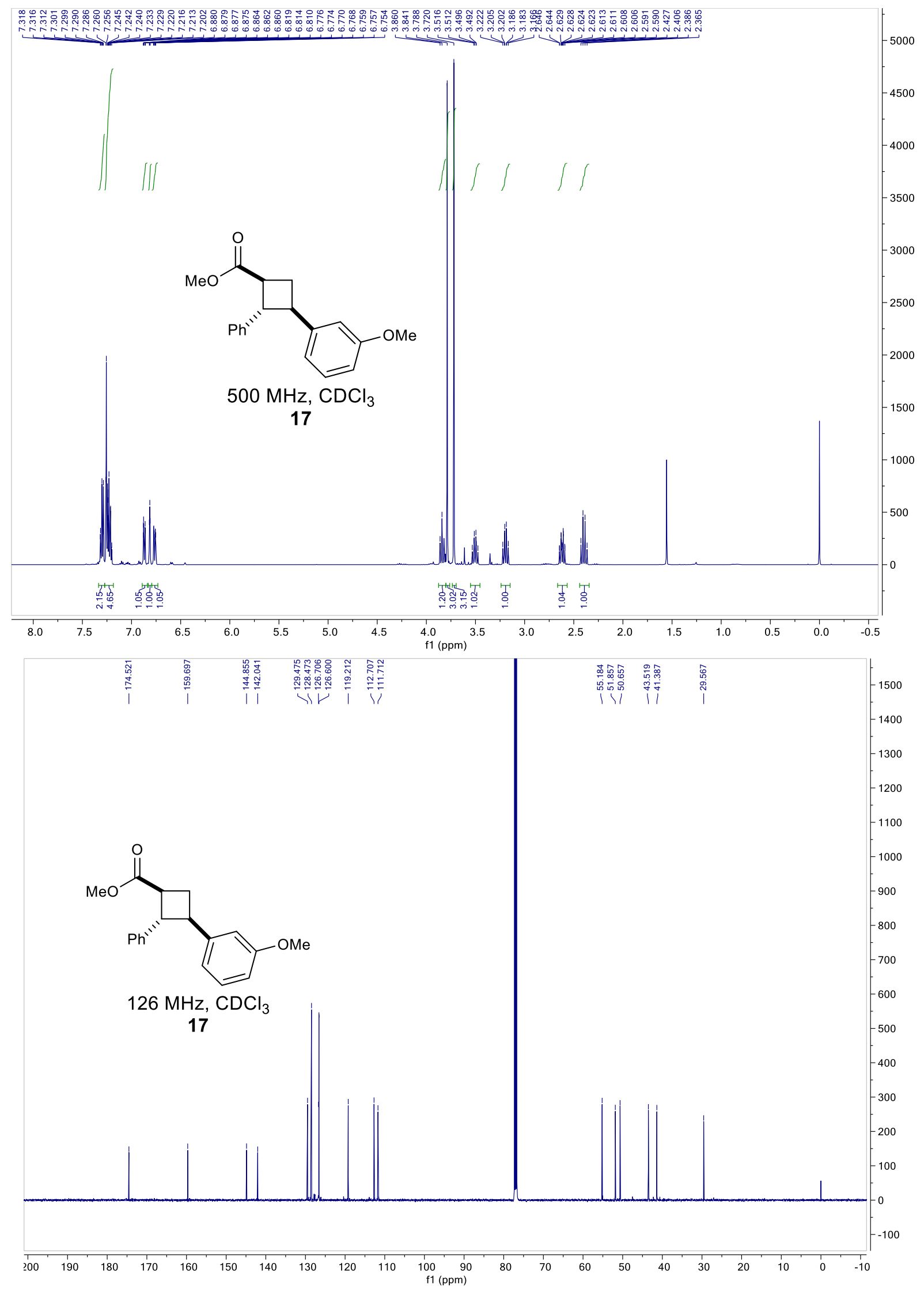




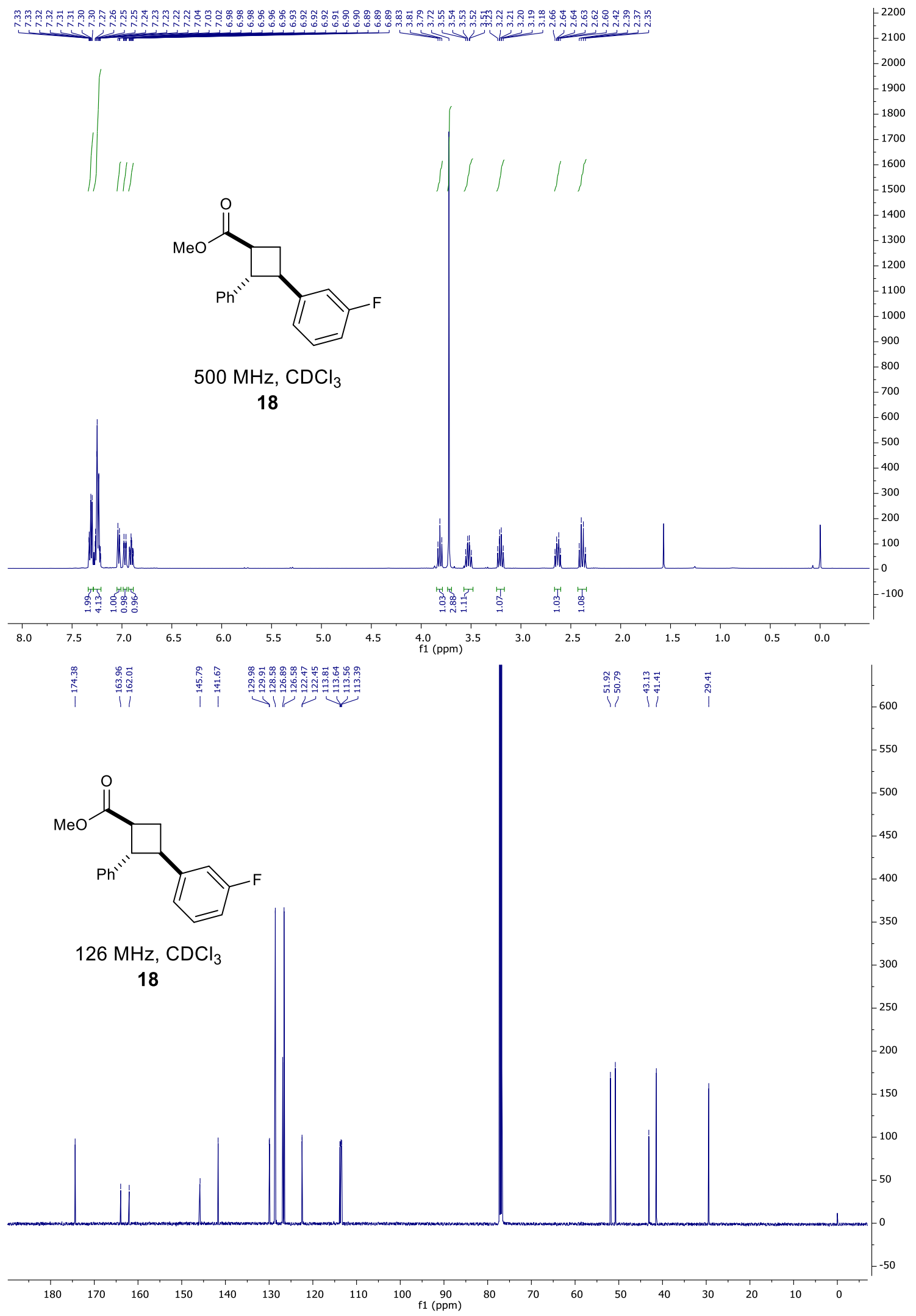



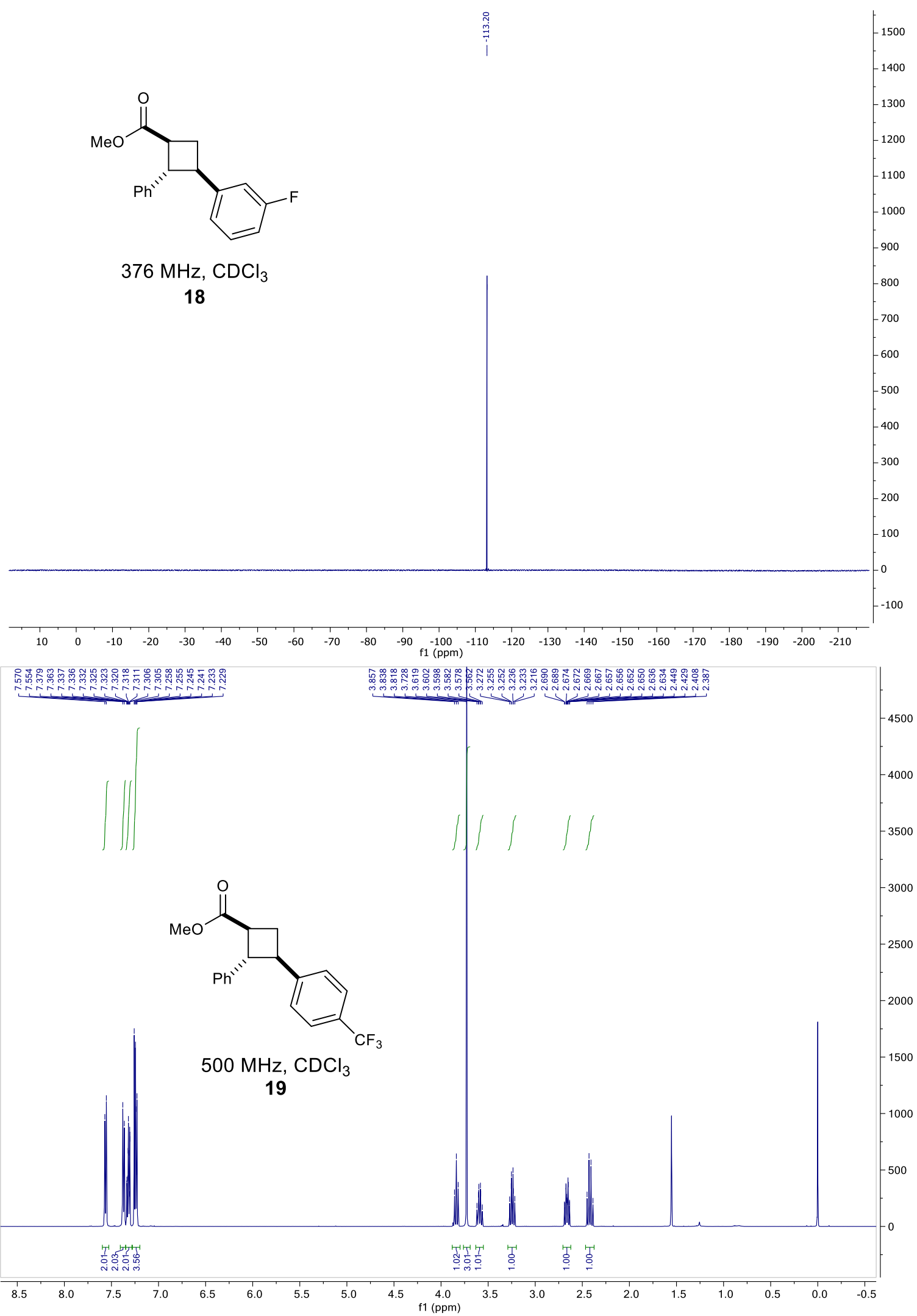


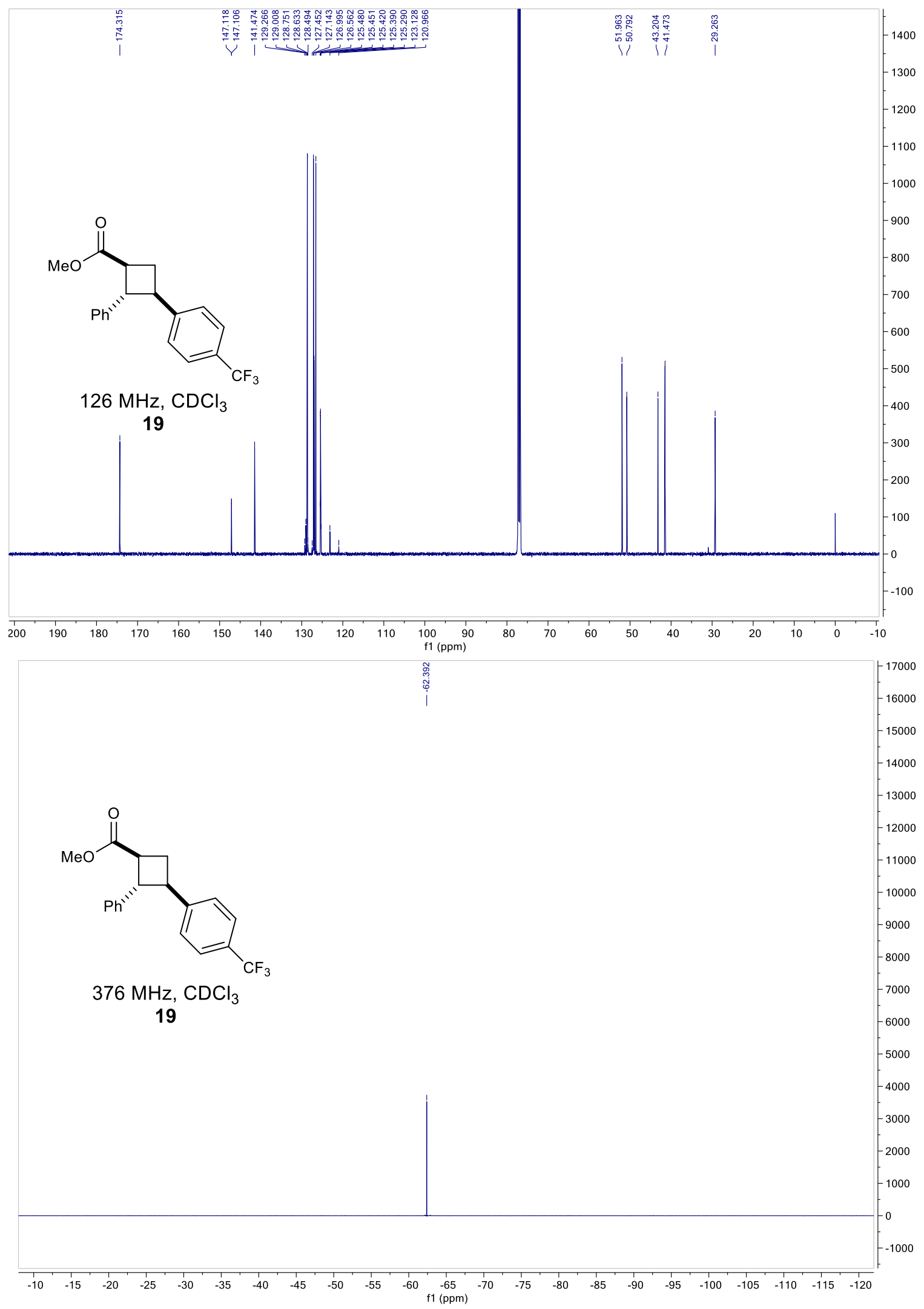




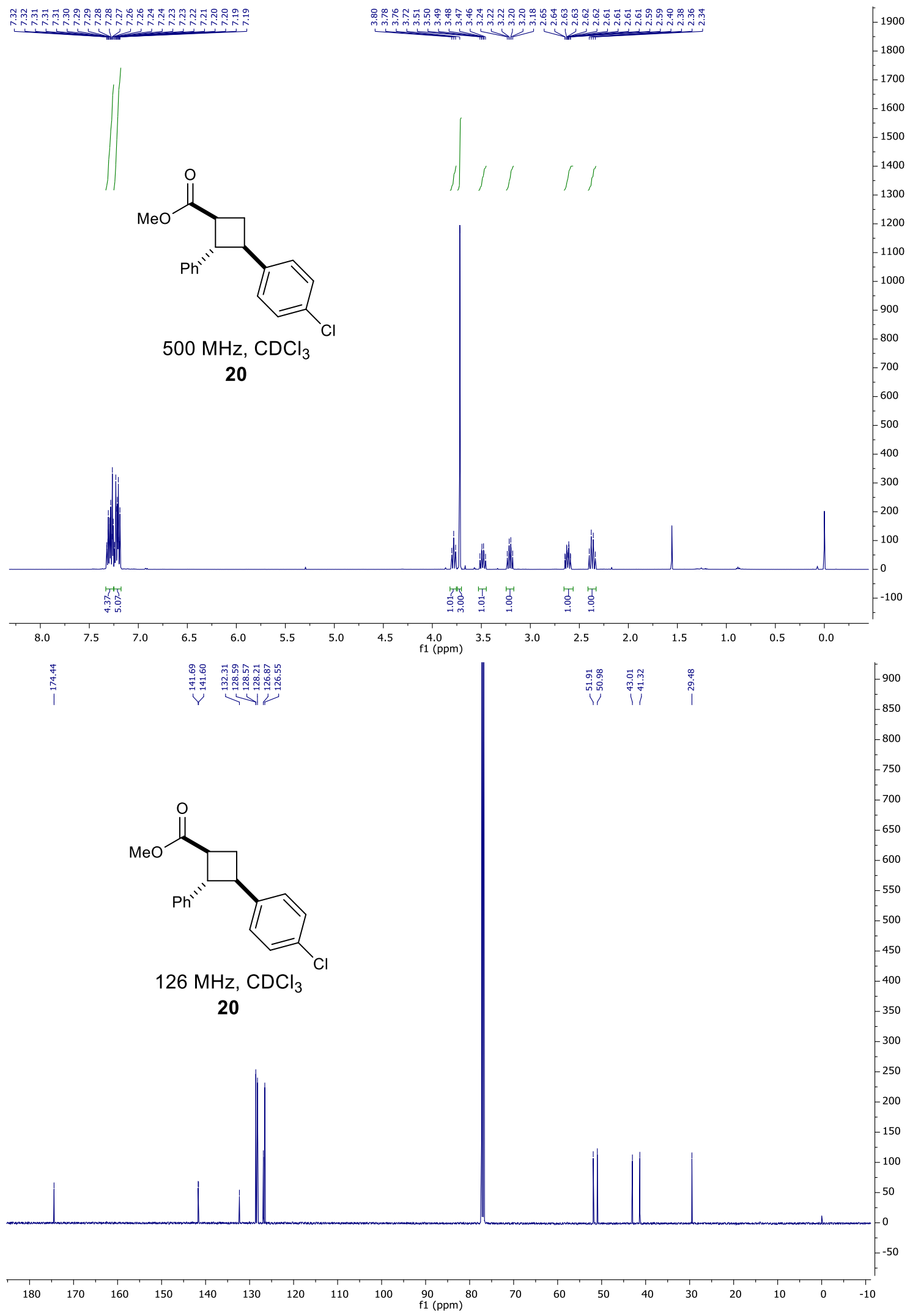




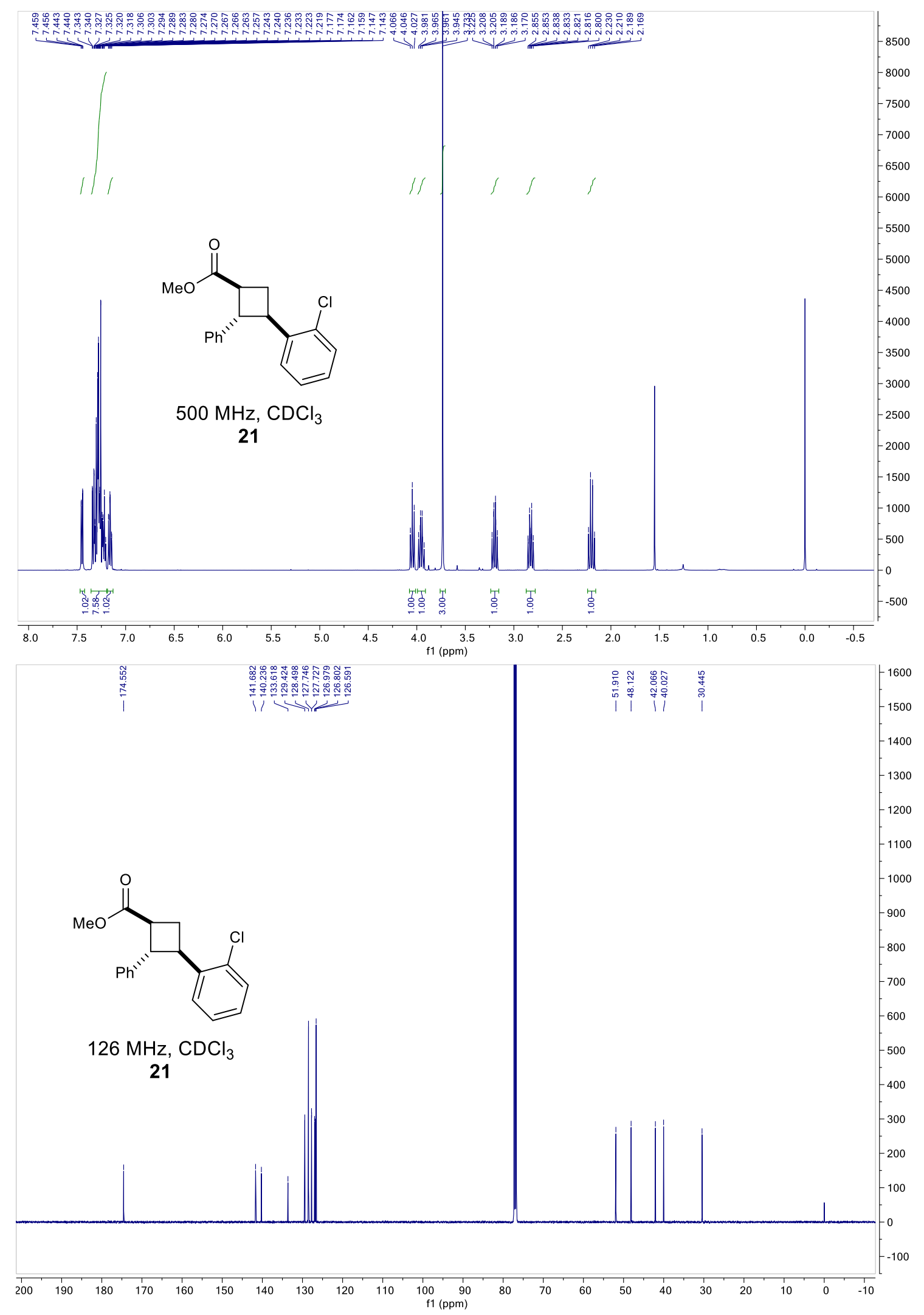




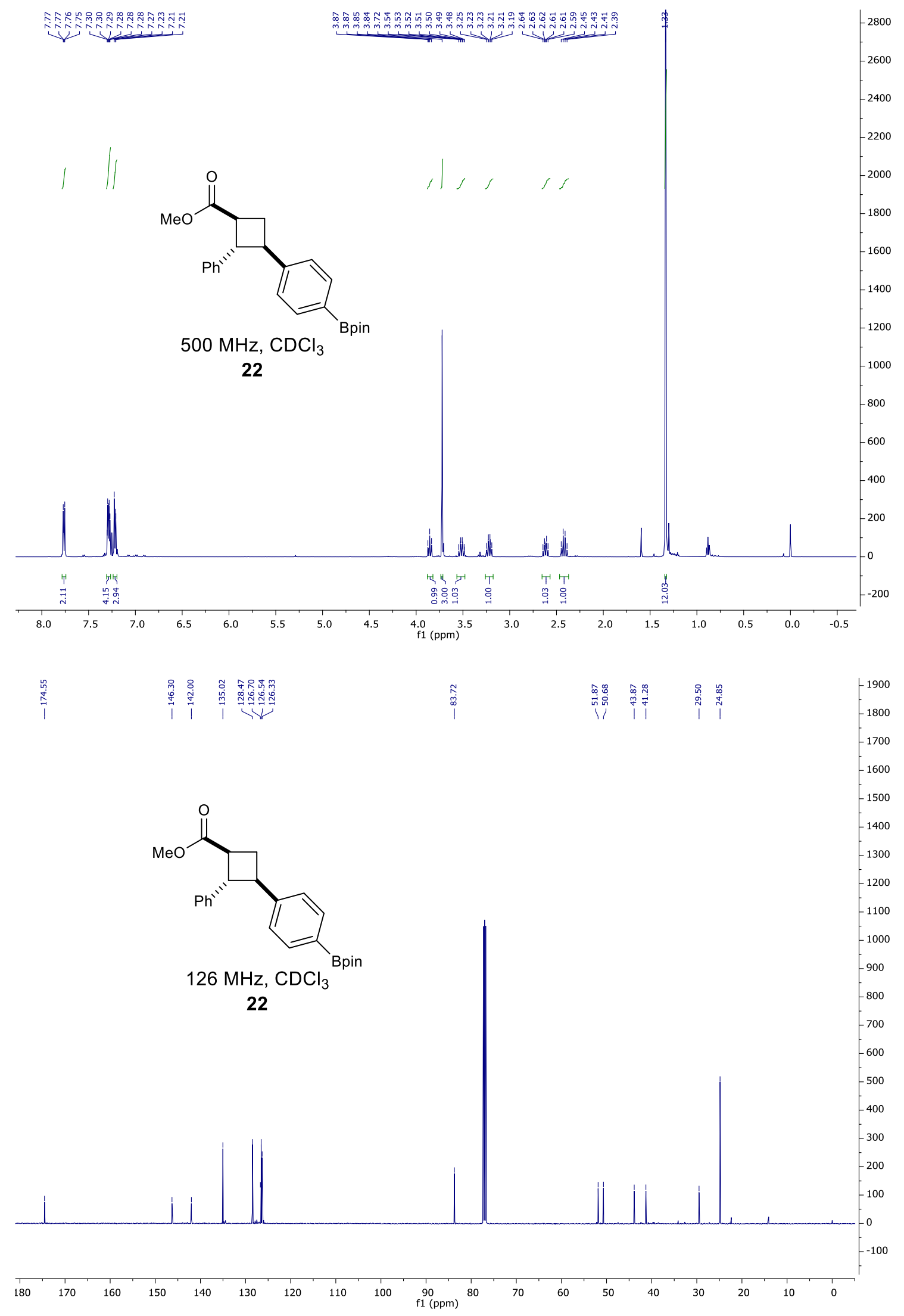




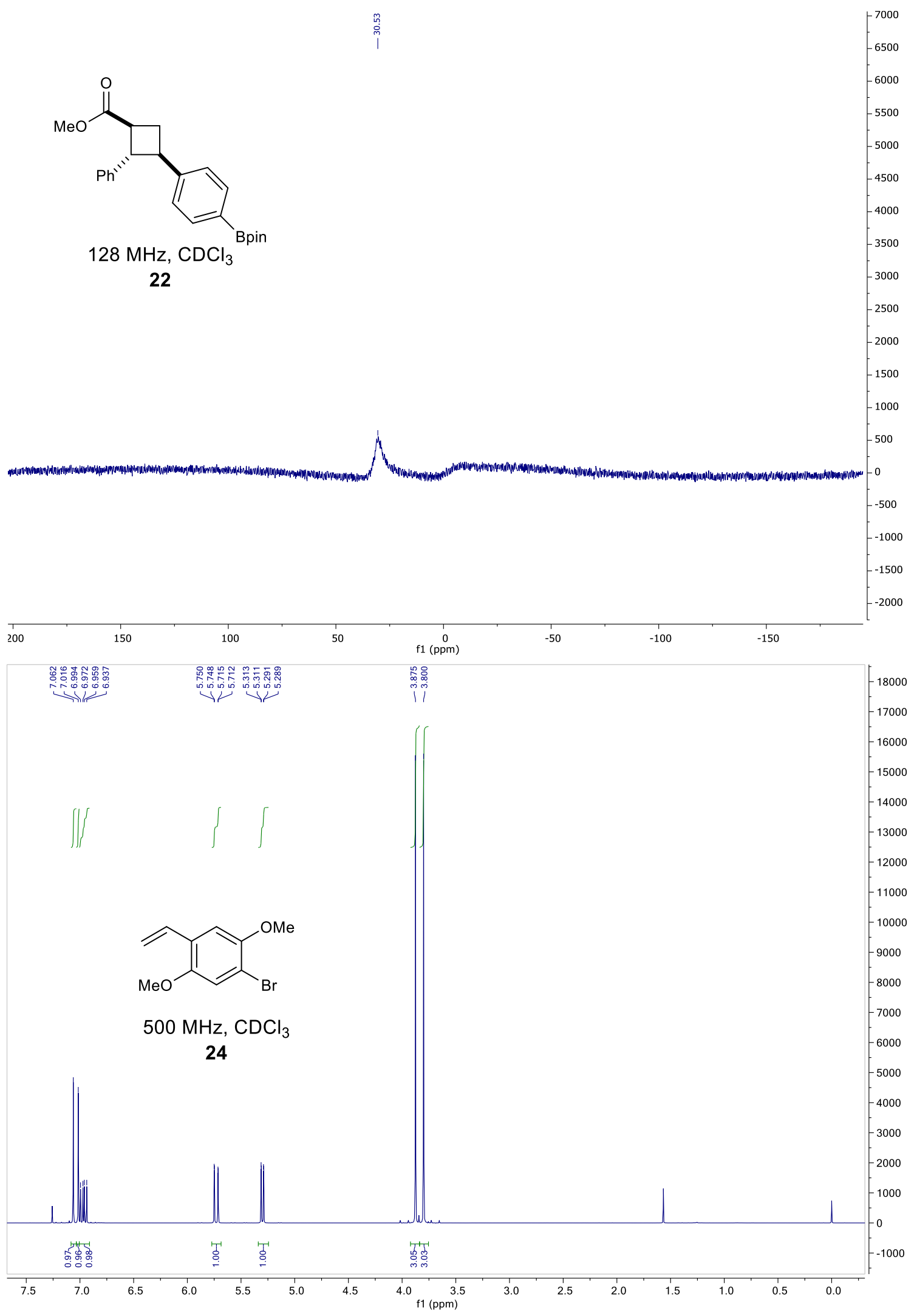




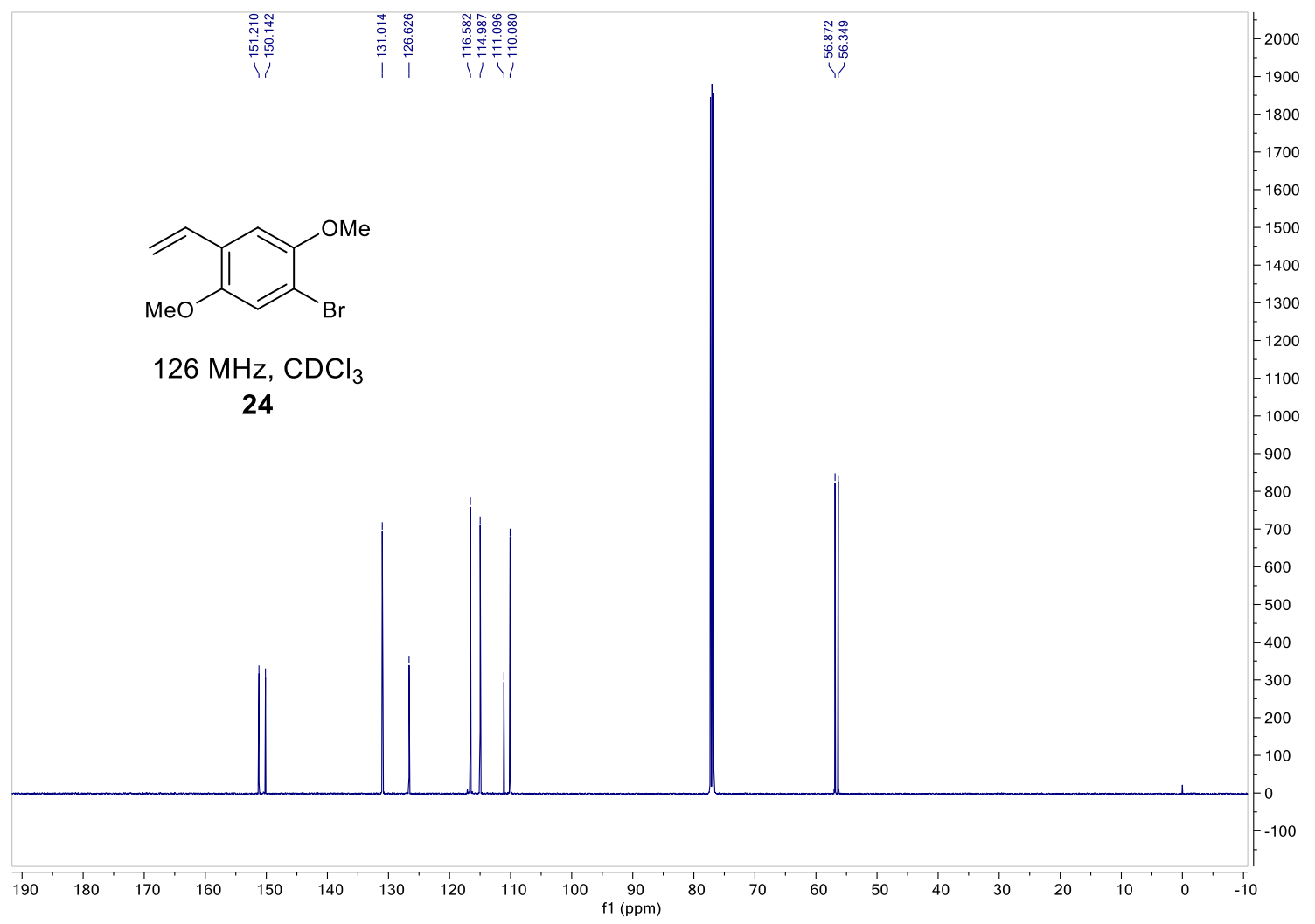




\section{Stern-Volmer Quenching Experiments}

Stern-Volmer experiments tracking the quenching of the phosphorescence of $\left[\operatorname{Ir}(\mathrm{ppy})_{2}(\mathrm{dtbpy})\right] \mathrm{PF}_{6}(\mathrm{Ir})$ were conducted on an ISS PC1 Spectrofluorimeter. For photocatalyst Ir, methyl cinnmate (4), and 4 with $\mathrm{EtAlCl}_{2}$, samples were prepared by adding degassed solutions of photocatalyst, quencher, and $\mathrm{CH}_{2} \mathrm{Cl}_{2}$. The solutions were loaded into quartz cuvettes and sealed under inert atmosphere by rubber septum. The concentration of photocatalyst Ir was $2.6 \times 10^{-5}$ M. Samples were irradiated at $420 \mathrm{~nm}$, and emission was detected at $536 \mathrm{~nm}$.

Table S1. Stern-Volmer quenching experiment with $\left[\operatorname{Ir}(\mathrm{ppy})_{2}(\mathrm{dtbpy})\right] \mathrm{PF}_{6}$ with varied [4] in $\mathrm{CH}_{2} \mathrm{Cl}_{2}$ at $23^{\circ} \mathrm{C}$.

\begin{tabular}{|c|c|c|}
\hline$[4] \mathbf{M}$ & $\mathbf{I}$ & $\mathbf{I}_{\mathbf{0}} / \mathbf{I}$ \\
\hline 0.000 & 197569 & 1.00 \\
0.007 & 103683 & 1.91 \\
0.014 & 76090 & 2.6 \\
0.020 & 60265 & 3.28 \\
0.025 & 51070 & 3.87 \\
0.030 & 44968 & 4.39 \\
\hline
\end{tabular}

Table S2. Stern-Volmer quenching experiment with $\left[\operatorname{Ir}(\mathrm{ppy})_{2}(\mathrm{dtbpy})\right] \mathrm{PF}_{6}$ with varied [4] w/ EtAlCl 2 in $\mathrm{CH}_{2} \mathrm{Cl}_{2}$ at $23{ }^{\circ} \mathrm{C}$. (1.0 equiv of $\mathrm{EtAlCl}_{2}$ was used)

\begin{tabular}{|c|c|c|}
\hline$\left[\mathbf{4} \mathbf{~ w} / \mathbf{E t A I C I}_{\mathbf{2}}\right] \mathbf{M}$ & $\mathbf{I}$ & $\mathbf{I}_{\mathbf{0}} / \mathbf{I}$ \\
\hline 0.000 & 168385 & 1.00 \\
0.007 & 39890 & 4.22 \\
0.014 & 23976 & 7.02 \\
0.020 & 17843 & 9.44 \\
0.025 & 15390 & 10.94 \\
0.030 & 13530 & 12.44 \\
\hline
\end{tabular}


Figure S1. Stern-Volmer plot of $\left[\operatorname{Ir}(\mathrm{ppy})_{2}(\mathrm{dtbpy})\right] \mathrm{PF}_{6}$ with varied [quencher] in $\mathrm{CH}_{2} \mathrm{Cl}_{2}$ at 23 ${ }^{\circ} \mathrm{C}$.

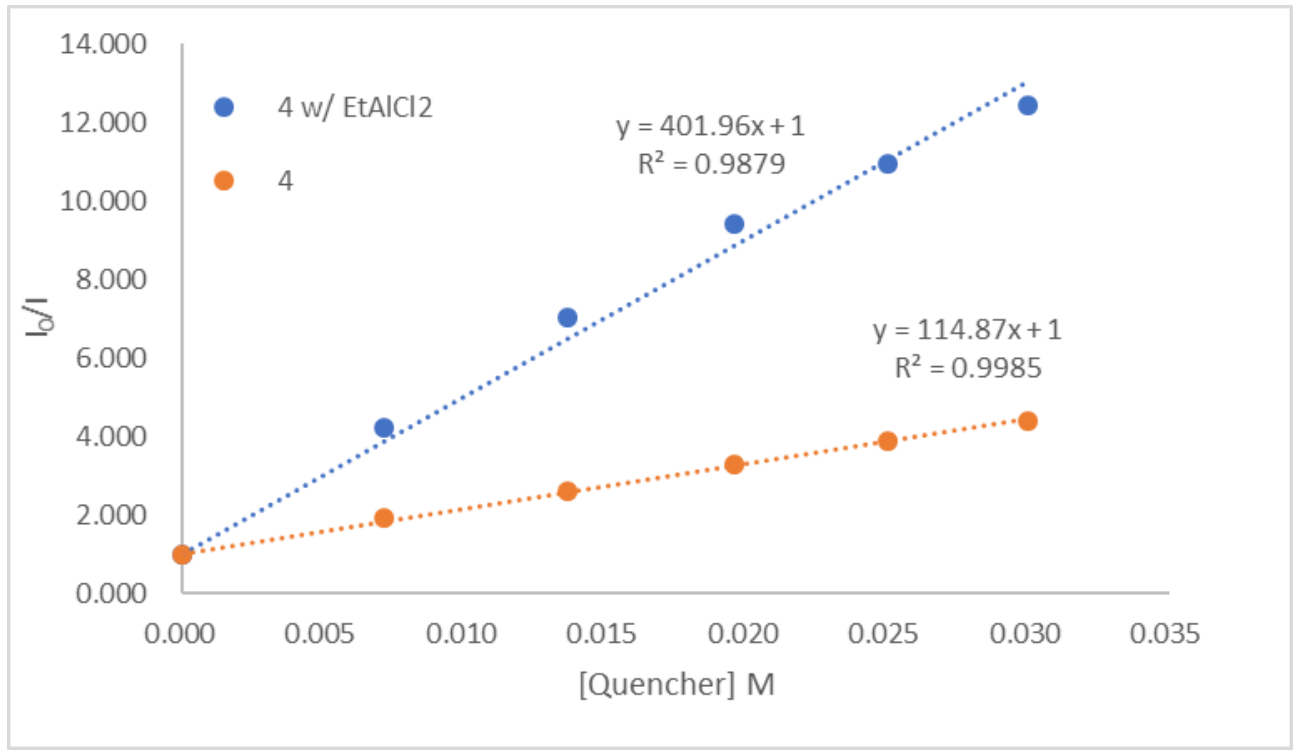




\section{Emission Spectra of Iridium(III) Photocatalysts}

The emission spectra were collected on an ISS PC1 Spectrofluorimeter. Samples were prepared by sparging solutions of each catalyst $\left(10 \mu \mathrm{M}\right.$ in $\mathrm{CH}_{2} \mathrm{Cl}_{2}$ ) with $\mathrm{N}_{2}$ for fifteen minutes in quartz cuvettes sealed by rubber septum prior to sample collection.

Figure S2. Emission Spectra of Iridium(III) Photocatalysts (410 nm excitation)

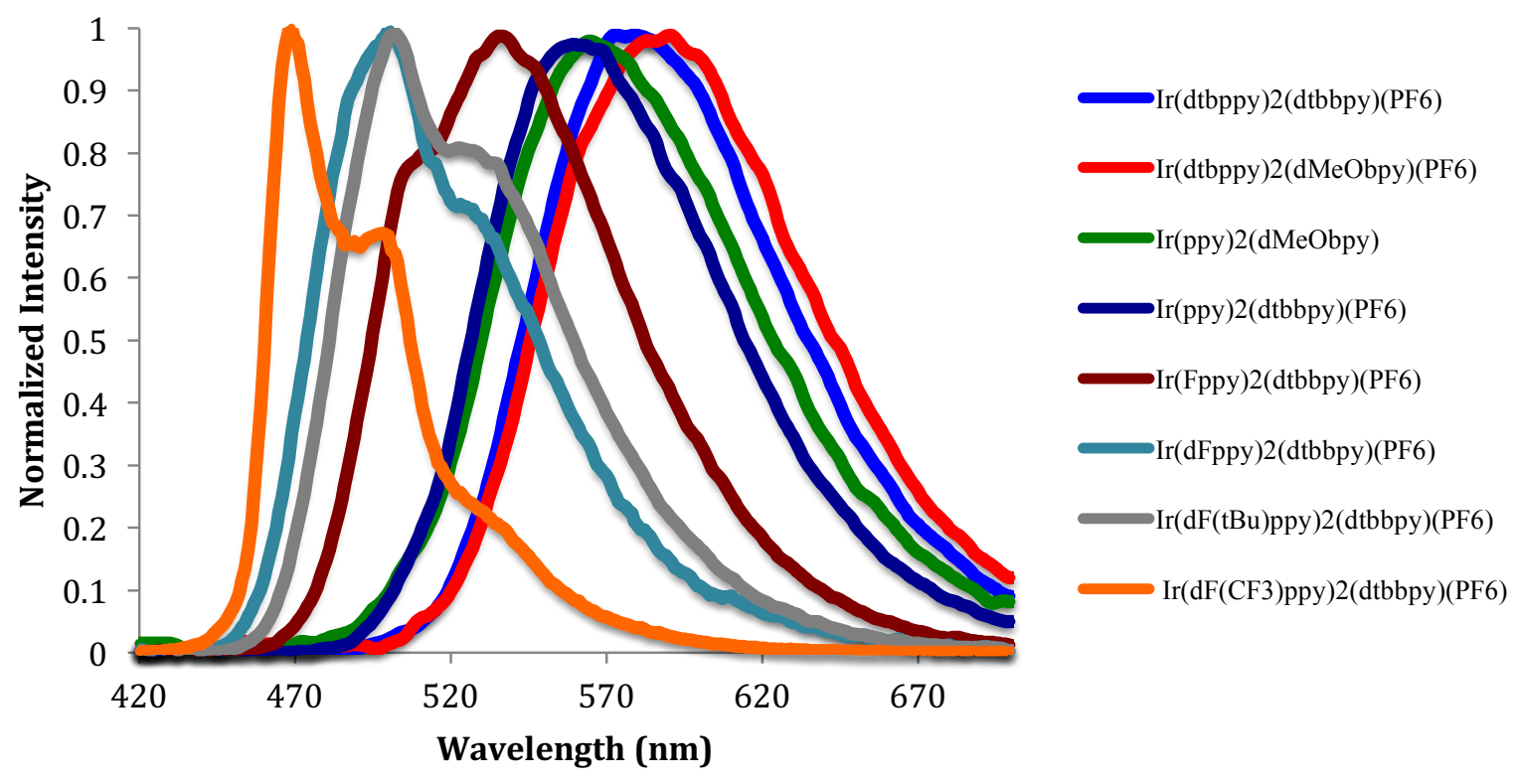




\section{Computational Details}

General Information. All computations were performed using density functional theory ${ }^{10}$ (DFT) implemented in Jaguar 9.1 suite. ${ }^{11}$ Geometry optimizations were conducted using B3LYP-D3 ${ }^{12}$ levels of theory with $6-31 \mathrm{G}^{* *}$ basis set. ${ }^{13}$ Ir metal was evaluated with Los Alamos LACVP $^{14}$ basis set, which reflects the relativistic effective core potentials. The energies of optimized structures were additionally reevaluated by single point calculations for each optimized geometry using B3LYP-D3 ${ }^{\text {xii }}$ levels of theory and cc-pVTZ(-f) ${ }^{15}$ basis set, which is a high quality triple- $\zeta$ basis set. Ir metal was calculated with LACV3P** basis set, with the decontracted LACVP exponents to match with the triple- $\zeta$ functions of the main group elements. $\mathrm{B}, \mathrm{Al}, \mathrm{Br}$ were also computed using LACV3P** instead of cc-pVTZ(-f) basis set. Vibrational frequencies were calculated with the optimized geometries at the same level as the geometry optimizations. Finally, solvation correction energies were evaluated by using the self-consistent reaction field (SCRF) approximations, ${ }^{16}$ that solve the linearized Poisson-Boltzmann equations with the proper dielectric constants ( $\varepsilon=9.08$ for dichloromethane). The solution phase Gibbs free energies were calculated as follows:

$$
\begin{aligned}
& \mathrm{G}(\text { Sol })=\mathrm{G}(\text { gas })+\mathrm{G}_{\text {solv }} \\
& \mathrm{G}(\text { gas })=\mathrm{H}(\text { gas })-\mathrm{TS}(\text { gas }) \\
& \mathrm{H}(\mathrm{Gas})=\mathrm{E}(\mathrm{SCF})+\mathrm{ZPE} \\
& \Delta \mathrm{G}(\text { Sol })=\Sigma \mathrm{G}(\text { Sol }) \text { for products }-\Sigma \mathrm{G}(\text { Sol }) \text { for reactants }
\end{aligned}
$$

$\mathrm{G}(\mathrm{Sol})$ is the solvation corrected Gibbs free energy from gas phase free energy $\mathrm{G}(\mathrm{gas})$; $\mathrm{H}(\mathrm{gas})$ is the enthalpy in the gas phase; $\mathrm{T}$ is the temperature $(298.15 \mathrm{~K})$; $\mathrm{S}($ gas $)$ is the entropy in the gas phase; $\mathrm{E}(\mathrm{SCF})$ is the electronic energy converged from the self-consistent field method; ZPE is the vibrational zero-point energy; and S for the vibrational entropy correction. Note that here 
entropy refers specifically to the vibrational/rotational/translational entropy of the solute(s). The solvent entropies are implicitly included in the continuum model.

Benchmark calculations for evaluating triplet energies. To ensure that our computational level is appropriate for calculating the triplet excited-state molecules, we compared the experimentally measured triplet energies with computational estimations. Triplet energies of photocatalysts utilized for this study were compared. (See Figure S2 for the emission spectra.) As shown in Table S3, the experimental values and computational estimations show both qualitative and quantitative agreement.

Table 3. Comparison of experimental triplet energies with computed values. (Energies in $\mathrm{kcal} / \mathrm{mol}$, evaluated with the computational level described above.)

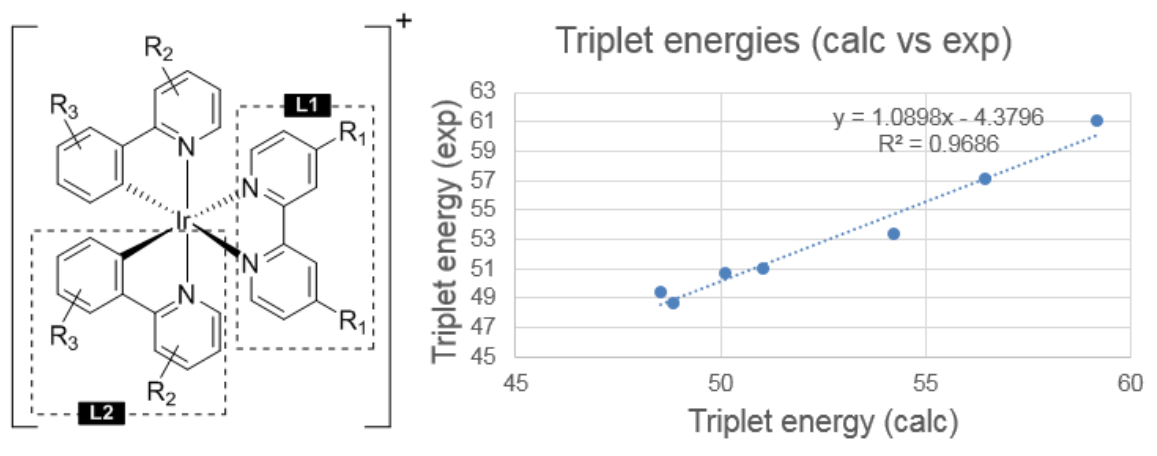

\begin{tabular}{|c|c|c|c|}
\hline L1 & L2 & $E_{T}(\exp )$ & $E_{T}$ (calc) \\
\hline $4,4^{\prime}-\left(^{\mathrm{t}} \mathrm{Bu}\right)_{2} \mathrm{bpy}$ & $\mathrm{dF}\left(\mathrm{CF}_{3}\right) \mathrm{ppy}$ & 61.0 & 59.21 \\
\hline $\left.4,4^{\prime}-{ }^{(t} \mathrm{Bu}\right)_{2} \mathrm{bpy}$ & $\mathrm{dF}\left({ }^{(B \mathrm{Bu}}\right) \mathrm{ppy}$ & 57.1 & 56.48 \\
\hline $4,4^{\prime}-\left(^{\mathrm{t}} \mathrm{Bu}\right)_{2} \mathrm{bpy}$ & Fppy & 53.3 & 54.25 \\
\hline $4,4^{\prime}-\left({ }^{+} \mathrm{Bu}\right)_{2} \mathrm{bpy}$ & ppy & 51.0 & 51.07 \\
\hline 4,4'-(MeO $)_{2}$ bpy & ppy & 50.6 & 50.13 \\
\hline $\left.4,4^{\prime}-{ }^{(} \mathrm{Bu}\right)_{2} \mathrm{bpy}$ & dtbppy & 49.4 & 48.54 \\
\hline 4,4'-(MeO $)_{2}$ bpy & dtbppy & 48.6 & 48.87 \\
\hline
\end{tabular}


Evaluating triplet energies of methyl cinnamate, Lewis acid bound adducts and photocatalyst. The triplet energies $\left(\mathrm{S}_{0}\right.$ to $\mathrm{T}_{1}$ energy gap) for substrate molecules and photocatalyst were determined by evaluating the energy difference between the singlet ground state structure and the first triplet state structure. The Gibbs free energy evaluated following the aforementioned method was compared. For the triplet state geometries of alkenes, two major conformers, planar and twisted structures, were found for most of the cases (Table S4). Energies of possible oxazaborolidine $\mathbf{6 f}$ bound complexes were also evaluated, and four different conformer structures and their energies are summarized in Table S5.

Table S4. Computed structures and energies for methyl cinnamate and Lewis acid bound adducts (Bond lengths in $\AA$, Energies are based on the singlet state of each geometry)

Singlet state (planar) Triplet state (planar) Triplet state (twisted)




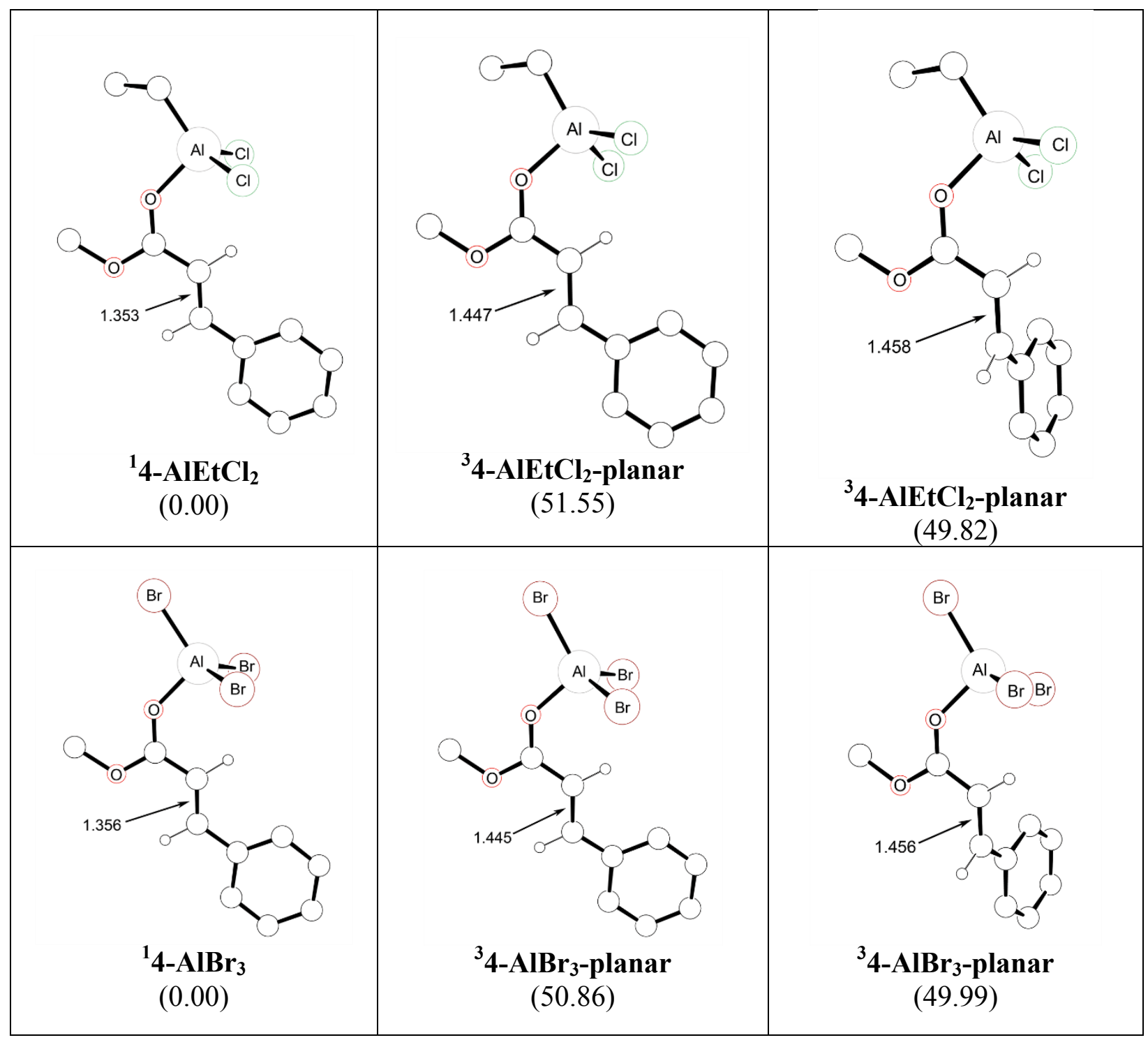

Table S5. Computed structures and energies for oxazaborolidine bound adducts (Bond lengths in $\AA$, Energies are based on the singlet state of each geometry) 


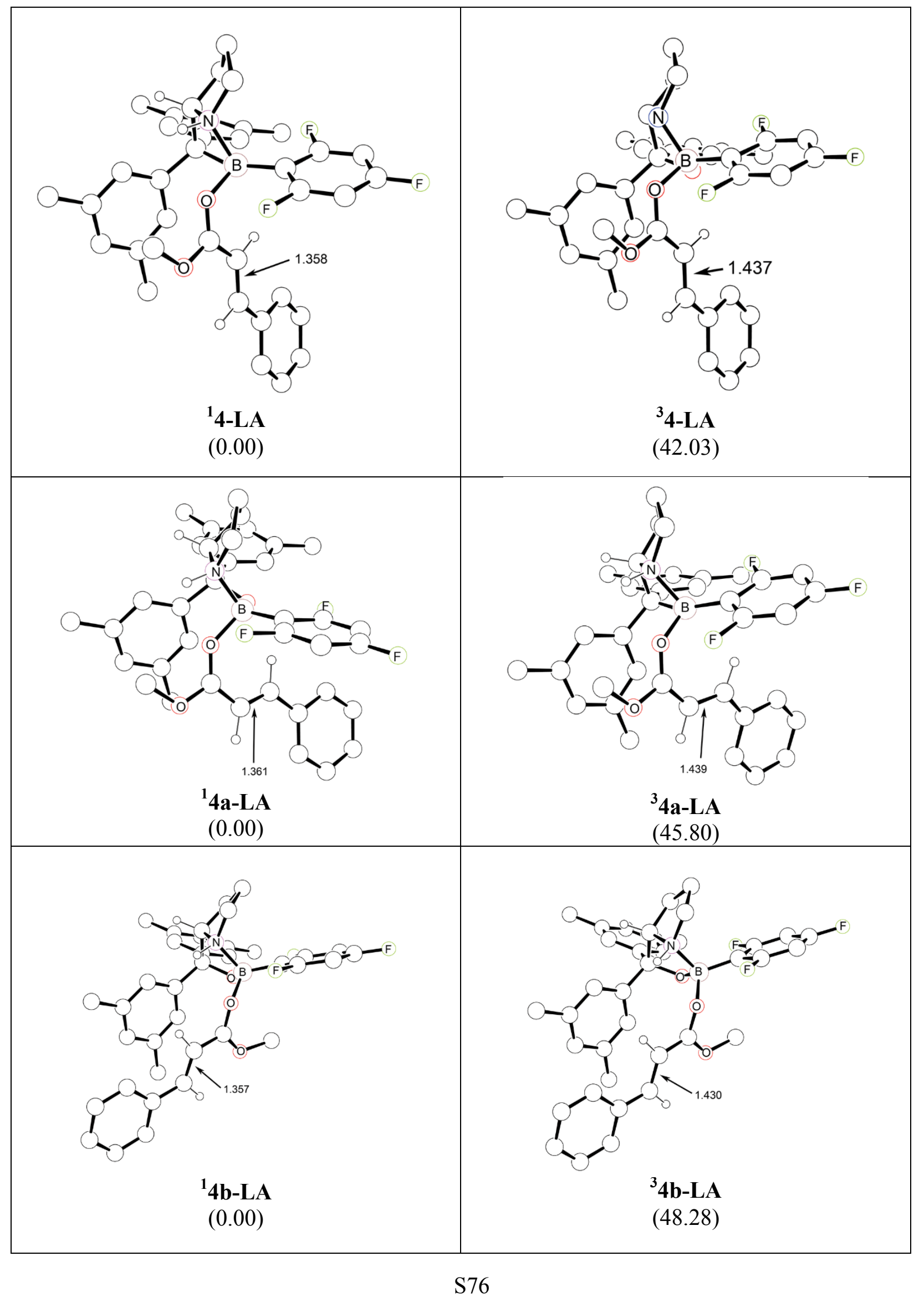




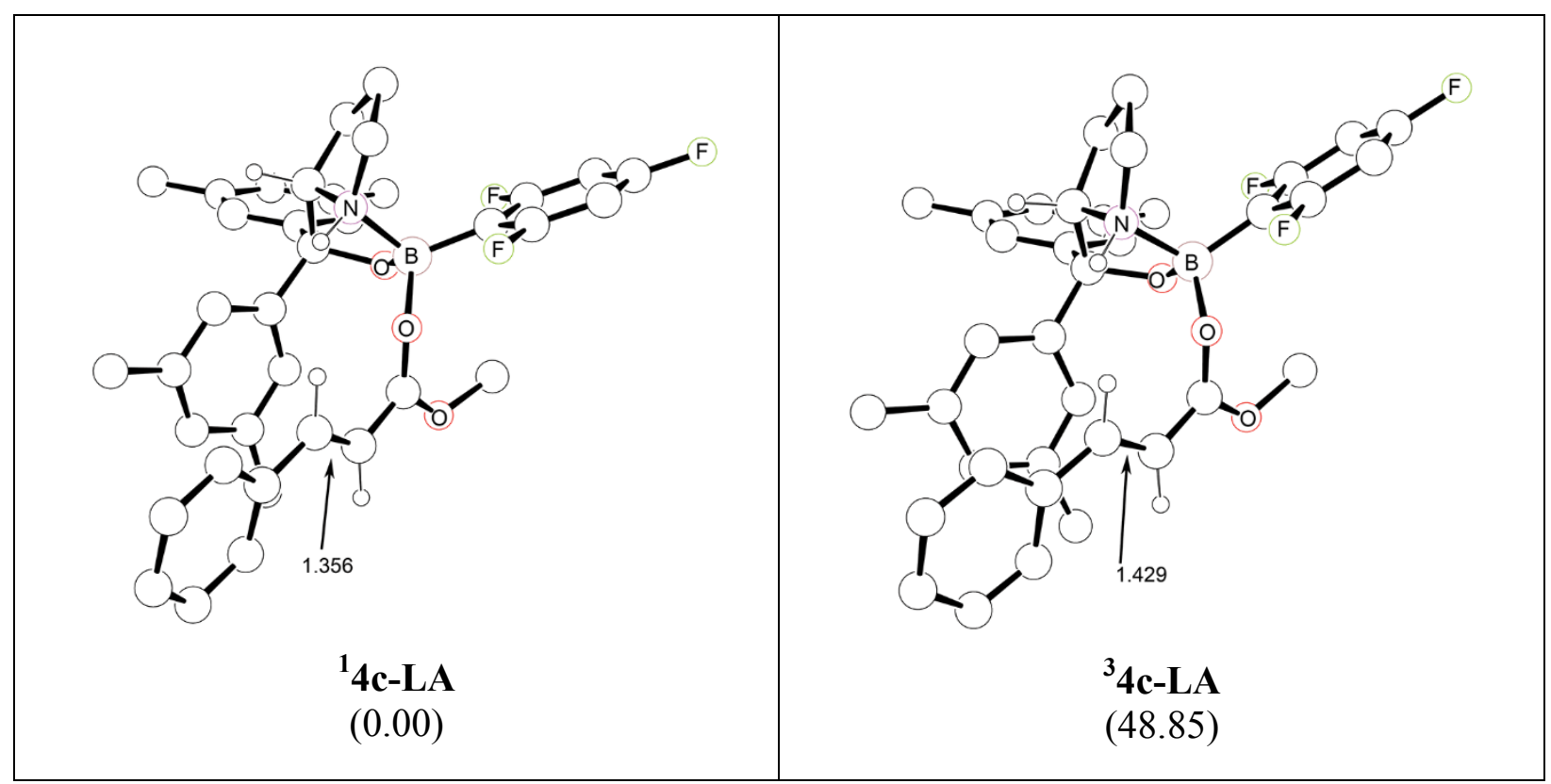

Frontier orbital analysis. For the frontier orbital analysis, Kohn-Sham orbitals were plotted with the DFT optimized geometry using B3LYP-D3 levels of theory and cc-pVTZ(-f) basis set, where several other atoms were computed using LACV3P** basis set as aforementioned. For the methyl cinnamate and the Lewis acid bound adducts, the $\pi$ and $\pi^{*}$ orbitals were found and their corresponding energies were calculated. For the photocatalyst, two SOMO energies were found to compare the frontier orbital energies.

Table S6. Frontier orbitals for methyl cinnamate 4 and Lewis acid bound adduct 4-LA

\begin{tabular}{|l|l|l|}
\hline & $1_{4}$ & $14-\mathrm{LA}$ \\
\hline
\end{tabular}




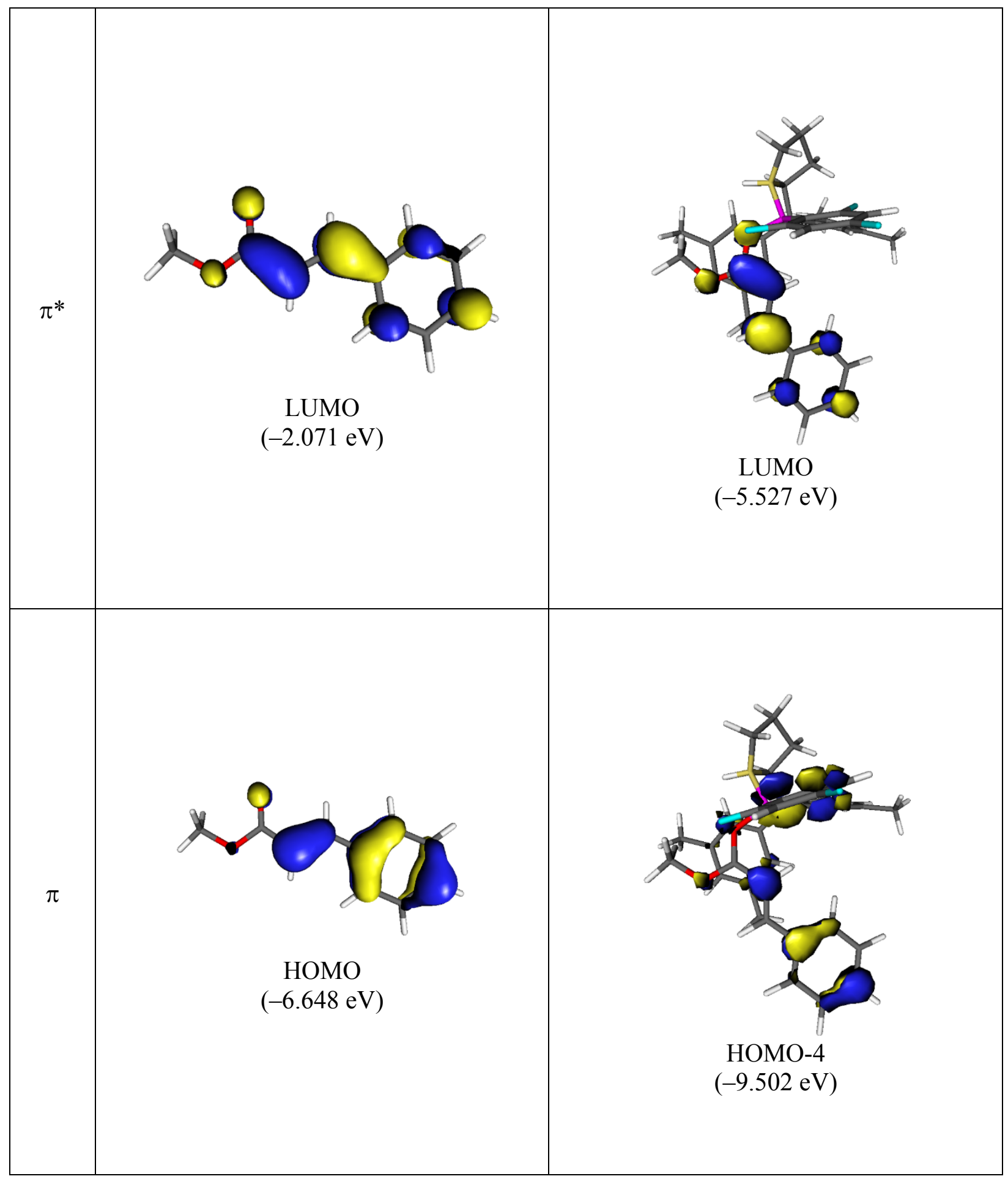

Energies, Coordinates, and Vibrational Frequencies of Optimized Structures.

Table S7. Computed energies of the optimized geometries 


\begin{tabular}{|c|c|c|c|c|}
\hline & $\mathrm{E}(\mathrm{SCF}) /(\mathrm{eV})$ & ZPE/(kcal/mol) & $\mathrm{S}($ gas)/(cal/mol•K) & G(solv)/(kcal/mol) \\
\hline & cc-pVTZ(-f)/LACV3P** & $6-31 \mathrm{G}^{* *} / \mathrm{LACVP} * *$ & $6-31 \mathrm{G}^{* *} / \mathrm{LACVP} * *$ & $6-31 \mathrm{G}^{* *} / \mathrm{LACVP} * *$ \\
\hline 6f & -40831.262 & 313.31 & 198.22 & -41.08 \\
\hline${ }^{1} \mathbf{7 f}$ & -56361.523 & 435.07 & 246.62 & -36.97 \\
\hline${ }^{3} \mathbf{7 f}$ & -56359.055 & 433.05 & 251.48 & -36.17 \\
\hline${ }^{1} 4$ & -14632.237 & 111.27 & 105.88 & -7.15 \\
\hline${ }^{3} 4$-planar & -14629.806 & 108.70 & 104.28 & -8.21 \\
\hline${ }^{3}$ 4-twisted & -14629.927 & 108.68 & 110.08 & -7.66 \\
\hline${ }^{1} 4-\mathrm{BF}_{3}$ & -23467.865 & 120.48 & 129.63 & -11.23 \\
\hline${ }^{3} 4-\mathrm{BF}_{3}$-planar & -23465.516 & 118.14 & 127.35 & -12.97 \\
\hline${ }^{3} 4-\mathrm{BF}_{3}$-twisted & -23465.605 & 118.29 & 129.90 & -10.23 \\
\hline${ }^{1} 4-\mathrm{AlEtCl}_{2}$ & -48439.277 & 155.47 & 155.74 & -11.19 \\
\hline${ }^{3} 4$-AlEtCl ${ }_{2}$-planar & -48436.961 & 153.26 & 150.26 & -12.48 \\
\hline${ }^{3} 4$-AIEtCl${ }_{2}$-twisted & -48437.027 & 153.08 & 157.30 & -10.40 \\
\hline${ }^{1} 4-\mathrm{AlBr}_{3}$ & -22311.592 & 114.89 & 153.48 & -12.49 \\
\hline${ }^{3} 4$-AlBr 3 -planar & -22309.293 & 112.71 & 148.63 & -13.91 \\
\hline${ }^{3} 4-\mathrm{AlBr}_{3}$-twisted & -22309.328 & 112.39 & 155.04 & -11.74 \\
\hline${ }^{1} 4-\mathrm{LA}$ & -55464.770 & 427.10 & 246.24 & -38.08 \\
\hline${ }^{3} 4-\mathrm{LA}$ & -55462.617 & 423.80 & 262.65 & -37.51 \\
\hline${ }^{1} 4 \mathrm{a}-\mathrm{LA}$ & -55464.633 & 425.71 & 255.32 & -37.75 \\
\hline${ }^{3} 4 \mathrm{a}-\mathrm{LA}$ & -55462.441 & 421.22 & 256.26 & -37.73 \\
\hline${ }^{1} 4 \mathrm{~b}-\mathrm{LA}$ & -55464.625 & 426.31 & 255.62 & -37.94 \\
\hline${ }^{3} 4 b-L A$ & -55462.445 & 424.39 & 256.33 & -37.80 \\
\hline${ }^{1} 4 \mathrm{c}-\mathrm{LA}$ & -55464.656 & 426.43 & 253.41 & -38.04 \\
\hline${ }^{3} 4 \mathrm{c}-\mathrm{LA}$ & -55462.492 & 424.77 & 252.86 & -37.60 \\
\hline
\end{tabular}


Table S8. Cartesian coordinates of the optimized geometries

\begin{tabular}{|c|c|c|c|}
\hline \multicolumn{4}{|l|}{$6 f$} \\
\hline B & -3.293317795 & 3.673214436 & 3.052648067 \\
\hline $\mathrm{N}$ & & & \\
\hline $\mathrm{O}$ & -1.960022211 & 3.650553226 & \\
\hline $\mathrm{C}$ & -4.258140087 & 2.723055840 & \\
\hline $\mathrm{C}$ & & & 4.80817699 \\
\hline $\mathrm{C}$ & -4.662101746 & 0.967052758 & 5.432527065 \\
\hline $\mathrm{H}$ & -4.303885460 & 0.345339835 & 6.242995739 \\
\hline $\mathrm{C}$ & & & 4.970142365 \\
\hline $\mathrm{C}$ & -6.4719 & & \\
\hline $\mathrm{H}$ & & & \\
\hline $\mathrm{C}$ & & & \\
\hline $\mathrm{C}$ & & & \\
\hline $\mathrm{C}$ & & & 30702 \\
\hline $\mathrm{C}$ & & & 2.949136972 \\
\hline $\mathrm{C}$ & & & \\
\hline $\mathrm{H}$ & & & \\
\hline $\mathrm{H}$ & & & 207 \\
\hline $\mathrm{H}$ & -5.6 & & \\
\hline $\mathrm{H}$ & -4.19 & & 489 \\
\hline $\mathrm{H}$ & & 7.80 & \\
\hline $\mathrm{H}$ & -2.16 & & \\
\hline $\mathrm{H}$ & -1.7619 & 7.49 & 51798 \\
\hline $\mathrm{C}$ & & & \\
\hline $\mathrm{C}$ & & & \\
\hline $\mathrm{H}$ & & & \\
\hline $\mathrm{C}$ & & & 1799 \\
\hline $\mathrm{C}$ & & & 550 \\
\hline $\mathrm{H}$ & & & 820 \\
\hline $\mathrm{C}$ & -0.32 & 1.533 & -0.13 \\
\hline $\mathrm{C}$ & -0.457 & & 94254 \\
\hline $\mathrm{H}$ & & & \\
\hline $\mathrm{C}$ & & & \\
\hline $\mathrm{C}$ & & & \\
\hline $\mathrm{C}$ & & & \\
\hline $\mathrm{H}$ & & & \\
\hline $\mathrm{C}$ & & & \\
\hline $\mathrm{C}$ & 2.353 & 6.215 & 04800 \\
\hline $\mathrm{H}$ & & & 3.451246500 \\
\hline $\mathrm{C}$ & & & \\
\hline $\mathrm{C}$ & & & \\
\hline $\mathrm{H}$ & -0.16 & & \\
\hline $\mathrm{H}$ & & & \\
\hline $\mathrm{C}$ & & & 0.84 \\
\hline $\mathrm{H}$ & & & \\
\hline $\mathrm{H}$ & 2.286896706 & 7.094190598 & -0.159396842 \\
\hline $\mathrm{H}$ & 2.749627352 & 8.220492363 & 1.125233769 \\
\hline $\mathrm{C}$ & 2.163769245 & 5.290101051 & 5.514005661 \\
\hline $\mathrm{H}$ & & 5.247800350 & \\
\hline & & 4.3467688 & \\
\hline & & & \\
\hline
\end{tabular}

C $\quad-2.596699476 \quad 3.097209215-2.783190966$

$\mathrm{H} \quad-1.927123785 \quad 3.008136272-3.644474030$

$\mathrm{H} \quad-3.386861563 \quad 2.348943949-2.912060738$

H $\quad-3.063360929 \quad 4.085774422 \quad-2.822823048$

$\begin{array}{llll}\text { C } & 0.572755873 & 0.327297568 & 0.006512278\end{array}$

$\begin{array}{llll}\mathrm{H} & 0.891435802 & 0.176658809 & 1.041001558\end{array}$

H $\quad 0.067002147-0.583001792-0.331186622$

$\mathrm{H} \quad 1.4737612010 .442531914-0.606741548$

F $\quad-6.042472363 \quad 3.312762022 \quad 2.323133230$

F $\quad-6.788980484-0.008550505 \quad 5.546583176$

F $\quad-2.573432684 \quad 2.001182079 \quad 5.236932755$

\begin{tabular}{lccc}
\hline$==========================$ \\
${ }^{1} 7 \mathbf{f}$ & & \\
$=========================$ \\
$\mathrm{Ir}$ & -0.168961421 & 0.033050727 & -0.511986434 \\
$\mathrm{C}$ & -1.128224373 & -4.631867886 & -1.412854671 \\
$\mathrm{C}$ & -0.938367963 & -4.278184414 & -0.077761181 \\
$\mathrm{C}$ & -0.585575759 & -2.960098267 & 0.216840252 \\
$\mathrm{~N}$ & -0.417533398 & -2.009333134 & -0.711155593 \\
$\mathrm{C}$ & -0.603551269 & -2.365092516 & -1.988731980 \\
$\mathrm{C}$ & -0.957226336 & -3.653954983 & -2.391659260 \\
$\mathrm{H}$ & -1.059784532 & -5.003747940 & 0.720450997 \\
$\mathrm{H}$ & -0.430158287 & -2.652369261 & 1.249540210 \\
$\mathrm{H}$ & -1.093729258 & -3.880145550 & -3.444527626 \\
$\mathrm{C}$ & 0.110470615 & -0.025225185 & -5.256567478 \\
$\mathrm{C}$ & -0.090272069 & -1.214439869 & -4.557222843 \\
$\mathrm{C}$ & -0.326270252 & -1.145601869 & -3.183116198 \\
$\mathrm{~N}$ & -0.371725142 & 0.000642906 & -2.492156029 \\
$\mathrm{C}$ & -0.176954150 & 1.134000421 & -3.178402424 \\
$\mathrm{C}$ & 0.066377647 & 1.177124143 & -4.552277565 \\
$\mathrm{H}$ & -0.065432936 & -2.175740004 & -5.060822487 \\
$\mathrm{H}$ & -0.217494130 & 2.055527210 & -2.600072384 \\
$\mathrm{H}$ & 0.216533989 & 2.129036188 & -5.051920891 \\
$\mathrm{C}$ & 4.487620831 & -1.538282514 & 0.184743553 \\
$\mathrm{C}$ & 3.426669836 & -2.434207916 & 0.060998708 \\
$\mathrm{C}$ & 2.164495945 & -1.929752707 & -0.256876647 \\
$\mathrm{~N}$ & 1.909889460 & -0.629459858 & -0.451255262 \\
$\mathrm{C}$ & 2.939486980 & 0.218065232 & -0.328784615 \\
$\mathrm{C}$ & 4.239724636 & -0.180900812 & -0.014440987 \\
$\mathrm{H}$ & 5.485400677 & -1.890066624 & 0.430923939 \\
$\mathrm{H}$ & 3.569201708 & -3.500424623 & 0.206580326 \\
$\mathrm{H}$ & 1.315531135 & -2.603415728 & -0.360596687 \\
$\mathrm{H}$ & 5.032686710 & 0.555491507 & 0.070790112 \\
$\mathrm{C}$ & 2.495793104 & 1.673085332 & -0.568893671 \\
$\mathrm{C}$ & 3.425125122 & 2.726480722 & -0.536785603 \\
$\mathrm{C}$ & 1.150132775 & 1.984968185 & -0.826301575 \\
$\mathrm{C}$ & 3.023844004 & 4.043517590 & -0.754069149 \\
$\mathrm{C}$ & 0.748240292 & 3.301805496 & -1.043662906 \\
$\mathrm{C}$ & 1.683312178 & 4.337480545 & -1.008575201 \\
$\mathrm{H}$ & 4.470248699 & 2.508909941 & -0.339697301 \\
$\mathrm{H}$ & 3.760171890 & 4.841609001 & -0.724435091 \\
$\mathrm{H}$ & -0.297685325 & 3.518988371 & -1.240838647 \\
$\mathrm{C}$ & -5.096726418 & 0.702975512 & -0.807334661 \\
& & & \\
& &
\end{tabular}


$\begin{array}{llll}\text { C } & -4.367164612 & 0.898404300 & -1.979153395\end{array}$

$\begin{array}{llll}\text { C } & -2.976615429 & 0.790136158 & -1.922107577\end{array}$

$\begin{array}{llll}\mathrm{N} & -2.297226667 & 0.508209467 & -0.803037763\end{array}$

$\begin{array}{llll}\text { C } & -3.012525082 & 0.323313594 & 0.313891321\end{array}$

$\begin{array}{llll}\text { C } & -4.404838085 & 0.408653915 & 0.366665781\end{array}$

$\begin{array}{lllll}\mathrm{H} & -6.180347919 & 0.778364897 & -0.808997869\end{array}$

H $\quad-4.860084057 \quad 1.129642129 \quad-2.918241501$

$\mathrm{H} \quad-2.378431797 \quad 0.937131464 \quad-2.819820881$

$\begin{array}{llll}\mathrm{H} & -4.927895546 & 0.248099416 & 1.304194570\end{array}$

$\begin{array}{llll}\text { C } & -2.053053141 & 0.068193898 & 1.491145492\end{array}$

$\begin{array}{llll}\text { C } & -2.539665937 & -0.078199916 & 2.801148653\end{array}$

$\begin{array}{llll}\text { C } & -0.666145742 & -0.024796918 & 1.285786748\end{array}$

$\begin{array}{llll}\text { C } & -1.671500564 & -0.309115618 & 3.866883516\end{array}$

$\begin{array}{llll}\text { C } & 0.202523008 & -0.255698264 & 2.351114511\end{array}$

$\begin{array}{llll}\text { C } & -0.296092540 & -0.398974717 & 3.646902800\end{array}$

$\begin{array}{llll}\mathrm{H} & -3.607667446 & -0.010070396 & 2.983344555\end{array}$

$\mathrm{H} \quad-2.070621252 \quad-0.419041753 \quad 4.871187210$

$\begin{array}{llll}\mathrm{H} & 1.271137953 & -0.323787928 & 2.168267488\end{array}$

$\begin{array}{llll}\text { C } & -1.518231034 & -6.071039200 & -1.797926784\end{array}$

$\begin{array}{llll}\text { C } & -2.119127274 & -6.841162205 & -0.607325554\end{array}$

C $-0.335377246-6.841162205-2.413933516$

$\begin{array}{lllll}\mathrm{H} & 0.071494445 & -6.313965321 & -3.282252550\end{array}$

H $\quad 0.477033645 \quad-6.967118740 \quad-1.689947367$

H $\quad-0.649834991 \quad-7.839670181 \quad-2.736697912$

$\begin{array}{lllll}\mathrm{H} & -1.384855747 & -6.967118740 & 0.195801347\end{array}$

H $\quad-2.445869207-7.839670181 \quad-0.917648256$

$\begin{array}{llll}\text { H } & -2.982195377 & -6.313965321 & -0.189431056\end{array}$

$\begin{array}{llll}\text { C } & 0.375085711 & -0.039420847 & -6.773596287\end{array}$

$\begin{array}{llll}\text { C } & -0.777822256 & -0.700905323 & -7.551374435\end{array}$

$\begin{array}{llll}\text { C } & 1.723232627 & -0.700905323 & -7.115116119\end{array}$

$\mathrm{H} \quad 2.549262524 \quad-0.210252285 \quad-6.591290474$

H $\quad 1.730100870 \quad-1.758825421 \quad-6.830879211$

$\begin{array}{lllll}\mathrm{H} & 1.919838309 & -0.645310044 & -8.191375732\end{array}$

H $\quad-0.880515695 \quad-1.758825421 \quad-7.286248684$

$\begin{array}{lllll}\mathrm{H} & -0.598440468 & -0.645310044 & -8.630638123\end{array}$

H $\quad-1.732416868 \quad-0.210252285 \quad-7.338142872$

$\begin{array}{llll}\text { C } & -2.611537933 & -5.966080666 & -2.877400637\end{array}$

$\mathrm{H} \quad-2.178121090 \quad-5.947772026-3.883841515$

$\mathrm{H} \quad-3.301456213 \quad-6.816331387 \quad-2.830562592$

$\begin{array}{lllll}\mathrm{H} & -3.204876423 & -5.052552223 & -2.756885767\end{array}$

C $\quad 0.454520792 \quad 1.429559231 \quad-7.228994846$

$\mathrm{H} \quad 1.482072592 \quad 1.807503939 \quad-7.179969311$

$\begin{array}{lllll}\mathrm{H} & 0.108906567 & 1.546173573 & -8.262464523\end{array}$

$\mathrm{H} \quad-0.165671155 \quad 2.076855659 \quad-6.598536491$

$\begin{array}{lllll}\text { F } & 0.563694119 & -0.627588451 & 4.701844692\end{array}$

F $\quad 1.285716414 \quad 5.641329765-1.223737836$
C $\quad-0.957226336-3.653954983-2.391659260$

$\begin{array}{llll}\mathrm{H} & -1.059784532 & -5.003747940 & 0.720450997\end{array}$

$\mathrm{H} \quad-0.430158287 \quad-2.652369261 \quad 1.249540210$

H $\quad-1.093729258 \quad-3.880145550-3.444527626$

C $\quad 0.110470615 \quad-0.025225185-5.256567478$

$\begin{array}{llll}\text { C } & -0.090272069 & -1.214439869 & -4.557222843\end{array}$

C $-0.326270252-1.145601869-3.183116198$

$\begin{array}{llll}\mathrm{N} & -0.371725142 & 0.000642906 & -2.492156029\end{array}$

$\begin{array}{llll}\text { C } & -0.176954150 & 1.134000421 & -3.178402424\end{array}$

$\begin{array}{lllll}\text { C } & 0.066377647 & 1.177124143 & -4.552277565\end{array}$

H $\quad-0.065432936-2.175740004-5.060822487$

$\mathrm{H} \quad-0.217494130 \quad 2.055527210 \quad-2.600072384$

H $\quad 0.2165339892 .129036188$-5.051920891

$\begin{array}{llll}\text { C } & 4.487620831 & -1.538282514 & 0.184743553\end{array}$

$\begin{array}{llll}\text { C } & 3.426669836 & -2.434207916 & 0.060998708\end{array}$

$\begin{array}{llll}\text { C } & 2.164495945 & -1.929752707 & -0.256876647\end{array}$

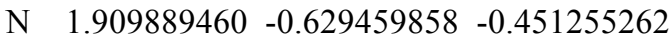

$\begin{array}{llll}\text { C } & 2.939486980 & 0.218065232 & -0.328784615\end{array}$

$\begin{array}{llll}\text { C } & 4.239724636 & -0.180900812 & -0.014440987\end{array}$

$\begin{array}{llll}\mathrm{H} & 5.485400677 & -1.890066624 & 0.430923939\end{array}$

H $\quad 3.569201708$-3.500424623 0.206580326

H $\quad 1.315531135-2.603415728-0.360596687$

$\begin{array}{llll}\mathrm{H} & 5.032686710 & 0.555491507 & 0.070790112\end{array}$

$\begin{array}{llll}\text { C } & 2.495793104 & 1.673085332 & -0.568893671\end{array}$

C $\quad 3.425125122 \quad 2.726480722 \quad-0.536785603$

C $\quad 1.150132775 \quad 1.984968185-0.826301575$

$\begin{array}{lllll}\text { C } & 3.023844004 & 4.043517590 & -0.754069149\end{array}$

C $\quad 0.748240292 \quad 3.301805496-1.043662906$

C $\quad 1.683312178 \quad 4.337480545-1.008575201$

H $\quad 4.470248699 \quad 2.508909941-0.339697301$

H $3.760171890 \quad 4.841609001-0.724435091$

H $\quad-0.297685325 \quad 3.518988371 \quad-1.240838647$

$\begin{array}{llll}\text { C } & -5.096726418 & 0.702975512 & -0.807334661\end{array}$

$\begin{array}{llll}\text { C } & -4.367164612 & 0.898404300 & -1.979153395\end{array}$

$\begin{array}{lllll}\text { C } & -2.976615429 & 0.790136158 & -1.922107577\end{array}$

$\begin{array}{llll}\mathrm{N} & -2.297226667 & 0.508209467 & -0.803037763\end{array}$

$\begin{array}{llll}\text { C } & -3.012525082 & 0.323313594 & 0.313891321\end{array}$

$\begin{array}{lllll}\text { C } & -4.404838085 & 0.408653915 & 0.366665781\end{array}$

$\begin{array}{lllll}\mathrm{H} & -6.180347919 & 0.778364897 & -0.808997869\end{array}$

H $\quad-4.860084057 \quad 1.129642129 \quad-2.918241501$

$\begin{array}{lllll}\mathrm{H} & -2.378431797 & 0.937131464 & -2.819820881\end{array}$

$\begin{array}{llll}\mathrm{H} & -4.927895546 & 0.248099416 & 1.304194570\end{array}$

$\begin{array}{llll}\text { C } & -2.053053141 & 0.068193898 & 1.491145492\end{array}$

$\begin{array}{llll}\text { C } & -2.539665937 & -0.078199916 & 2.801148653\end{array}$

$\begin{array}{llll}\text { C } & -0.666145742 & -0.024796918 & 1.285786748\end{array}$

$\begin{array}{llll}\text { C } & -1.671500564 & -0.309115618 & 3.866883516\end{array}$

$\begin{array}{llll}\text { C } & 0.202523008 & -0.255698264 & 2.351114511\end{array}$

$\begin{array}{llll}\text { C } & -0.296092540 & -0.398974717 & 3.646902800\end{array}$

$\begin{array}{llll}\mathrm{H} & -3.607667446 & -0.010070396 & 2.983344555\end{array}$

$\mathrm{H} \quad-2.070621252 \quad-0.419041753 \quad 4.871187210$

H $\quad 1.271137953 \quad-0.323787928 \quad 2.168267488$

C $\quad-1.518231034-6.071039200-1.797926784$

C $-2.119127274-6.841162205-0.607325554$

C $-0.335377246-6.841162205-2.413933516$

H $\quad 0.071494445 \quad-6.313965321 \quad-3.282252550$

H $\quad 0.477033645 \quad-6.967118740-1.689947367$ 
$\begin{array}{lllll}\mathrm{H} & -0.649834991 & -7.839670181 & -2.736697912\end{array}$

$\begin{array}{lllll}\mathrm{H} & -1.384855747 & -6.967118740 & 0.195801347\end{array}$

$\begin{array}{lllll}\mathrm{H} & -2.445869207 & -7.839670181 & -0.917648256\end{array}$

H $\quad-2.982195377 \quad-6.313965321 \quad-0.189431056$

$\begin{array}{llll}\text { C } & 0.375085711 & -0.039420847 & -6.773596287\end{array}$

$\begin{array}{llll}\text { C } & -0.777822256 & -0.700905323 & -7.551374435\end{array}$

$\begin{array}{llll}\text { C } & 1.723232627 & -0.700905323 & -7.115116119\end{array}$

H $\quad 2.549262524 \quad-0.210252285 \quad-6.591290474$

H $\quad 1.730100870 \quad-1.758825421-6.830879211$

H $\quad 1.919838309 \quad-0.645310044 \quad-8.191375732$

$\begin{array}{lllll}\mathrm{H} & -0.880515695 & -1.758825421 & -7.286248684\end{array}$

$\begin{array}{lllll}\mathrm{H} & -0.598440468 & -0.645310044 & -8.630638123\end{array}$

$\begin{array}{lllll}\mathrm{H} & -1.732416868 & -0.210252285 & -7.338142872\end{array}$

$\begin{array}{llll}\text { C } & -2.611537933 & -5.966080666 & -2.877400637\end{array}$

H $\quad-2.178121090 \quad-5.947772026-3.883841515$

H $\quad-3.301456213-6.816331387-2.830562592$

H $\quad-3.204876423 \quad-5.052552223 \quad-2.756885767$

$\begin{array}{llll}\text { C } & 0.454520792 & 1.429559231 & -7.228994846\end{array}$

H $\quad 1.482072592 \quad 1.807503939 \quad-7.179969311$

$\mathrm{H} \quad 0.108906567 \quad 1.546173573 \quad-8.262464523$

$\mathrm{H} \quad-0.165671155 \quad 2.076855659 \quad-6.598536491$

$\begin{array}{lllll}\text { F } & 0.563694119 & -0.627588451 & 4.701844692\end{array}$

F $\quad 1.285716414 \quad 5.641329765-1.223737836$

\begin{tabular}{|c|c|c|c|}
\hline \multicolumn{4}{|l|}{${ }^{1} 4$} \\
\hline $\mathrm{C}$ & -4.034955502 & 1.166083813 & 0.59937006 \\
\hline $\mathrm{C}$ & -2.734083176 & 0.468350142 & 6120110 \\
\hline $\mathrm{O}$ & -4.335605621 & 2.132335424 & 1.276850700 \\
\hline $\mathrm{O}$ & -4.886715412 & 0.583125591 & -0.280367017 \\
\hline $\mathrm{C}$ & -6.184391022 & 1.191751719 & -0.359877795 \\
\hline $\mathrm{C}$ & -1.751270056 & 0.889366925 & 1.422311187 \\
\hline $\mathrm{C}$ & -0.407142341 & 0.330995619 & 96343 \\
\hline $\mathrm{C}$ & & & \\
\hline $\mathrm{H}$ & & & 76738 \\
\hline $\mathrm{C}$ & 1.748 & 0.41 & 7674 \\
\hline $\mathrm{H}$ & 2.398879290 & 0.870 & 9576 \\
\hline $\mathrm{C}$ & 2.203059673 & -0.660534859 & 84457 \\
\hline $\mathrm{H}$ & 3.209173203 & -1.044896483 & 22196 \\
\hline $\mathrm{C}$ & 1.356898308 & -1.240051985 & 13562 \\
\hline $\mathrm{H}$ & 1.706515551 & -2.075358868 & 0.388722032 \\
\hline $\mathrm{C}$ & 0.068175361 & -0.751640737 & 0.804436386 \\
\hline $\mathrm{H}$ & -0.573808253 & -1.210974455 & 589520 \\
\hline $\mathrm{H}$ & -2.628512383 & -0.376136422 & -0.066627473 \\
\hline $\mathrm{H}$ & -6.741592884 & 0.608916521 & -1.093616247 \\
\hline $\mathrm{H}$ & -6.104275227 & 2.235080481 & -0.678361118 \\
\hline $\mathrm{H}$ & -6.684680462 & 1.165907264 & 0.612393439 \\
\hline & $1.9044 \pi$ & $1 . / 4 / 35450 /$ & \\
\hline
\end{tabular}

$\begin{array}{llll}\text { O } & -4.885736465 & 0.554617822 & -0.321502030\end{array}$

$\begin{array}{llll}\text { C } & -6.177476883 & 1.167542100 & -0.391800314\end{array}$

$\begin{array}{llll}\text { C } & -1.699777842 & 0.936031103 & 1.505454540\end{array}$

$\begin{array}{llll}\text { C } & -0.435258746 & 0.387834847 & 1.613142729\end{array}$

$\begin{array}{llll}\mathrm{C} & 0.511675656 & 0.938302517 & 2.565581083\end{array}$

$\begin{array}{llll}\mathrm{H} & 0.192961395 & 1.778012395 & 3.176334381\end{array}$

C $\quad 1.774197221 \quad 0.419624776 \quad 2.702513456$

$\begin{array}{llll}\mathrm{H} & 2.460547209 & 0.851598918 & 3.425471783\end{array}$

$\begin{array}{llll}\text { C } & 2.194113255 & -0.676701725 & 1.910276771\end{array}$

H $\quad 3.194729328-1.0812113292 .024304152$

C $\quad 1.302930832-1.236522436 \quad 0.974548817$

H $\quad 1.625041246-2.076946974 \quad 0.365889847$

$\begin{array}{llll}\text { C } & 0.028785439 & -0.734467030 & 0.818283617\end{array}$

$\begin{array}{lllll}\mathrm{H} & -0.635600388 & -1.184272170 & 0.089615256\end{array}$

H $-2.600111008-0.372180760-0.069271848$

$\mathrm{H} \quad-6.738168240 \quad 0.595943630-1.132076383$

$\mathrm{H} \quad-6.097777367 \quad 2.215306282 \quad-0.697910488$

$\begin{array}{llll}\mathrm{H} & -6.680449963 & 1.132946014 & 0.579651177\end{array}$

H $\quad-1.972291708 \quad 1.776438832 \quad 2.136028290$

\begin{tabular}{|c|c|c|}
\hline${ }^{3} 4$-planar & & \\
\hline C -4.030510902 & 1.128441334 & 0.570985794 \\
\hline C -2.751111031 & 0.468457192 & 0.596843541 \\
\hline O $\quad-4.335701942$ & 2.097563267 & 1.266432524 \\
\hline
\end{tabular}

\begin{tabular}{|c|c|c|c|}
\hline \multicolumn{4}{|c|}{${ }^{3} 4$-twisted } \\
\hline $\mathrm{C}$ & -4.066656113 & 1.178953409 & \\
\hline $\mathrm{C}$ & -2.76418 & & \\
\hline $\mathrm{O}$ & -4.392555714 & 2.148786306 & 1.23420667 \\
\hline $\mathrm{O}$ & -4.898595810 & 0.569634080 & -0.323675573 \\
\hline $\mathrm{C}$ & -6.204473019 & & 771901 \\
\hline $\mathrm{C}$ & -1.69 & & 25 \\
\hline $\mathrm{C}$ & -0.73 & 1.96 & 82 \\
\hline $\mathrm{C}$ & 0.28 & 2.32 & 2.0 \\
\hline $\mathrm{H}$ & 0.310 & 1.82 & 0535 \\
\hline $\mathrm{C}$ & & & \\
\hline $\mathrm{H}$ & 2.007499456 & 3.53 & 57212 \\
\hline $\mathrm{C}$ & 1.2118834 & 3.91 & 92389 \\
\hline $\mathrm{H}$ & & & 38094 \\
\hline $\mathrm{C}$ & 0.21 & & \\
\hline $\mathrm{H}$ & 0.18 & 4.07 & \\
\hline $\mathrm{C}$ & -0.742 & 2.62 & -0.1 \\
\hline $\mathrm{H}$ & -1.516 & 2.379 & -0.8 \\
\hline $\mathrm{H}$ & -2.617 & -0.32 & -0.0 \\
\hline & -6.742940903 & 0.557641864 & -1.159203649 \\
\hline & -6.139263630 & 2.197720051 & -0.747346044 \\
\hline & -6.716734886 & 1.127100587 & \\
\hline & -1.648221970 & 0.535203695 & 2.485686779 \\
\hline
\end{tabular}


C $\quad-0.917391002-2.392923117-0.913320363$

H $\quad-1.735715747 \quad-2.705240011 \quad-1.557154894$

C $\quad 0.287729174 \quad-3.089253187 \quad-0.937505305$

H $\quad 0.408086389-3.942297459-1.598499537$

C $\quad 1.337431431 \quad-2.687462091 \quad-0.110091664$

H $\quad 2.279201508 \quad-3.228160858 \quad-0.124956325$

C $\quad 1.175563455-1.587855220 \quad 0.740462482$

H $1.991788983 \quad-1.276943922 \quad 1.385331392$

$\begin{array}{llll}\text { C } & -0.026177082 & -0.890611291 & 0.765869141\end{array}$

H $-0.138341904-0.042110022 \quad 1.432672381$

$\begin{array}{lllll}\mathrm{H} & -2.045300961 & 0.990332425 & 1.314285398\end{array}$

H $\quad-6.695195198 \quad 0.580240846 \quad-1.221529841$

H $\quad-6.100273132 \quad 2.214887381 \quad-0.767640352$

$\begin{array}{lllll}\mathrm{H} & -6.696296215 & 1.110705376 & 0.496792883\end{array}$

H $\quad-3.123621464-1.014086366 \quad-0.761009037$

$\begin{array}{lllll}\text { B } & -3.525192499 & 2.795957804 & 2.433112860\end{array}$

F $\quad-4.346637726 \quad 3.785938025 \quad 2.852532148$

F $\quad-2.369249582 \quad 3.204391479 \quad 1.818697572$

F $\quad-3.339626551 \quad 1.758700967 \quad 3.307346106$

\section{${ }^{3} 4-\mathrm{BF}_{3}$-planar}

$\begin{array}{llll}\text { C } & -4.051611423 & 1.114816666 & 0.547770977\end{array}$

$\begin{array}{llll}\text { C } & -2.790526390 & 0.455004513 & 0.651096761\end{array}$

$\begin{array}{llll}\text { O } & -4.416010380 & 2.114303827 & 1.232331276\end{array}$

$\begin{array}{llll}\mathrm{O} & -4.894606590 & 0.608173847 & -0.357869655\end{array}$

$\begin{array}{llll}\text { C } & -6.180987358 & 1.250007153 & -0.475163817\end{array}$

$\begin{array}{llll}\text { C } & -2.429032087 & -0.708937585 & -0.141701072\end{array}$

C $-1.178879738-1.308980703-0.103122681$

$\begin{array}{llll}\text { C } & -0.913927138 & -2.469273090 & -0.931628048\end{array}$

H $\quad-1.713001370-2.837679148-1.569773197$

$\begin{array}{lllll}\text { C } & 0.307666034 & -3.092402220 & -0.915680528\end{array}$

H $\quad 0.480268270 \quad-3.960967541 \quad-1.544676542$

$\begin{array}{llll}\text { C } & 1.351377249 & -2.613927841 & -0.084187239\end{array}$

H $\quad 2.314654112 \quad-3.113952875 \quad-0.076362193$

C $\quad 1.130585670-1.486432552 \quad 0.731667399$

$\mathrm{H} \quad 1.932161450 \quad-1.120676398 \quad 1.367814779$

C $-0.086849540 \quad-0.842637599 \quad 0.734251380$

$\begin{array}{llll}\mathrm{H} & -0.231316254 & 0.023170328 & 1.369590521\end{array}$

$\begin{array}{llll}\mathrm{H} & -2.107995749 & 0.882176101 & 1.371284008\end{array}$

H $\quad-6.7105116840 .691695988$ - 1.246569157

$\begin{array}{lllll}\mathrm{H} & -6.057218552 & 2.296521425 & -0.765655398\end{array}$

$\begin{array}{lllll}\mathrm{H} & -6.715241432 & 1.204697132 & 0.477425158\end{array}$

$\begin{array}{lllll}\text { H } & -3.187723637 & -1.116618156 & -0.801512837\end{array}$

$\begin{array}{llll}\text { B } & -3.481746435 & 2.776484489 & 2.433527231\end{array}$

F $\quad-4.248436928 \quad 3.801388025 \quad 2.876380682$

$\begin{array}{llll}\text { F } & -2.318163872 & 3.141584873 & 1.795593381\end{array}$

F $\quad-3.300222158 \quad 1.741875529 \quad 3.317180395$

\begin{tabular}{|c|c|c|}
\hline${ }^{3} 4-\mathrm{BF}_{3}$-twisted & & \\
\hline C -4.259595394 & 0.882700503 & 0.772653341 \\
\hline C -3.026149750 & 0.139598772 & 0.893123567 \\
\hline O -4.471663475 & 2.012938023 & 1.279994488 \\
\hline
\end{tabular}

$\begin{array}{llll}\text { O } & -5.210434914 & 0.287466139 & 0.069221079\end{array}$

C $\quad-6.446762562 \quad 1.016504765-0.105767220$

$\begin{array}{llll}\text { C } & -2.688412189 & -0.968397617 & 0.009094629\end{array}$

$\begin{array}{llll}\text { C } & -1.956626177 & -0.783057451 & -1.189995885\end{array}$

$\begin{array}{llll}\text { C } & -1.657095671 & -1.892428517 & -2.031369925\end{array}$

H $\quad-1.995197296-2.882896185-1.738337517$

C $\quad-0.947405219-1.718265772-3.208396912$

H $\quad-0.728064120 \quad-2.576255560-3.837508917$

C $-0.510703623-0.441408217-3.590041876$

H $\quad 0.047145985-0.309719026-4.512238979$

C $\quad-0.793507159 \quad 0.663100660 \quad-2.774492025$

$\mathrm{H} \quad-0.451716453 \quad 1.652147293 \quad-3.065601110$

C $\quad-1.503525376 \quad 0.507232130-1.593849063$

H $\quad-1.7079893351 .367847204-0.962485492$

$\begin{array}{llll}\mathrm{H} & -2.335402727 & 0.517054081 & 1.642442465\end{array}$

H $\quad-7.070608616 \quad 0.371501356-0.722414017$

H $\quad-6.251932621 \quad 1.969262600-0.603019118$

$\begin{array}{llll}\mathrm{H} & -6.913507938 & 1.204004169 & 0.863793135\end{array}$

H $\quad-3.006295681 \quad-1.972060442 \quad 0.288017839$

$\begin{array}{llll}\text { B } & -3.218802691 & 2.965904713 & 1.850879788\end{array}$

$\begin{array}{llll}\text { F } & -3.833153486 & 4.143627644 & 2.097023249\end{array}$

$\begin{array}{llll}\text { F } & -2.335042238 & 2.964209080 & 0.793674648\end{array}$

$\begin{array}{llll}\text { F } & -2.749236584 & 2.293704271 & 2.949516535\end{array}$ 
H $\quad-4.564636707 \quad 5.662736893 \quad 1.226805925$

H $\quad-5.771444321 \quad 5.993624687 \quad 2.471406460$

H $\quad-5.719445705 \quad 4.411852837 \quad 1.689542294$

\begin{tabular}{|c|c|c|c|}
\hline \multicolumn{4}{|c|}{${ }^{3} 4$-AIEtCl 2 -planar } \\
\hline $\mathrm{C}$ & -3.826173306 & 1.137689829 & 0.811054349 \\
\hline $\mathrm{C}$ & -2.596303463 & 0.421519458 & 0.790220022 \\
\hline $\mathrm{O}$ & -4.055213451 & 2.157265425 & 1.536391139 \\
\hline $\mathrm{O}$ & -4.786429405 & 0.678445280 & 0.005072795 \\
\hline $\mathrm{C}$ & -6.028091431 & 1.415158272 & -0.021219745 \\
\hline $\mathrm{C}$ & -2.328057528 & -0.720385730 & -0.057443399 \\
\hline $\mathrm{C}$ & -1.091099501 & -1.354095697 & -0.109137006 \\
\hline $\mathrm{C}$ & -0.910286844 & & -0.973951519 \\
\hline $\mathrm{H}$ & -1.756174445 & -2.8 & 51676 \\
\hline $\mathrm{C}$ & 0.2912 & -3.15 & $-1 .($ \\
\hline $\mathrm{H}$ & 0.401 & -4.02( & 2990 \\
\hline $\mathrm{C}$ & & & -0.26 \\
\hline $\mathrm{H}$ & 2.342291117 & -3.253073 & -0.324724734 \\
\hline $\mathrm{C}$ & 1.257078409 & -1.607852936 & 0.579458058 \\
\hline $\mathrm{H}$ & 2.10483551 & & 1.170192003 \\
\hline $\mathrm{C}$ & & & \\
\hline $\mathrm{H}$ & -0.01 & & \\
\hline $\mathrm{H}$ & -1.84 & 0.77 & 6648 \\
\hline $\mathrm{H}$ & -6.654 & 0.898 & -0.7 \\
\hline $\mathrm{H}$ & -5.8463754 & 2.45 & -0.32 \\
\hline $\mathrm{H}$ & -6.493973732 & 1.405934572 & 0.968387723 \\
\hline $\mathrm{Al}$ & -2.882095814 & 3.281667948 & 2.514492035 \\
\hline $\mathrm{Cl}$ & -1.417459249 & 3.722436666 & 0.964886546 \\
\hline $\mathrm{Cl}$ & -2.01723218 & 1.930 & 3.982 \\
\hline $\mathrm{C}$ & -4.0116481 & 4.749 & 3.15 \\
\hline $\mathrm{H}$ & -3.1358778 & -1.087 & \\
\hline $\mathrm{C}$ & -4.949803352 & 5.3244 & 83793 \\
\hline $\mathrm{H}$ & -3.376910925 & 5.551126003 & 3.553015709 \\
\hline $\mathrm{H}$ & -4.604599476 & 4.390805244 & 4.005466938 \\
\hline $\mathrm{H}$ & -4.385263920 & 5.724614143 & 1.217896581 \\
\hline $\mathrm{H}$ & -5.577448845 & 6.139706612 & 2.453305960 \\
\hline $\mathrm{H}$ & -5.625560760 & 4.554802418 & $1.67478525 t$ \\
\hline
\end{tabular}

C $\quad-0.446106076 \quad 0.596431851 \quad-2.872110367$

H $\quad-0.025913142 \quad 1.550808311 \quad-3.175599813$

C $\quad-1.136938334 \quad 0.507023990 \quad-1.674128294$

H $-1.249663711 \quad 1.387120366-1.047519803$

H $-1.860572815 \quad 0.531481564 \quad 1.631947041$

H $\quad-6.630378246 \quad 0.918857098 \quad-0.654345751$

H $\quad-5.774183273 \quad 2.424920559-0.167946562$

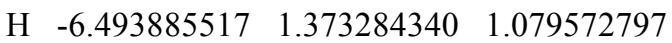

Al $\quad-2.795644760 \quad 3.311959505 \quad 2.399189234$

$\begin{array}{lllll}\mathrm{Cl} & -1.614102364 & 3.662595034 & 0.603863001\end{array}$

$\begin{array}{lllll}\mathrm{Cl} & -1.684523463 & 2.173196793 & 3.863440514\end{array}$

C $-3.948778391 \quad 4.774466038 \quad 2.999946594$

H $\quad-2.799381733 \quad-1.831755996 \quad 0.253367275$

C $\quad \begin{array}{llll}5.032860279 & 5.174538136 & 1.976676583\end{array}$

H $\quad-3.325368881 \quad 5.647287369 \quad 3.236257792$

H $\quad-4.421511173 \quad 4.487661362 \quad 3.949633121$

H $\quad-4.591342449 \quad 5.489787579 \quad 1.023875117$

$\begin{array}{lllll}\mathrm{H} & -5.655496120 & 6.004440784 & 2.335009098\end{array}$

H $\quad-5.707459927 \quad 4.336654663 \quad 1.760705709$

\begin{tabular}{|c|c|c|}
\hline${ }^{3} 4-\mathrm{AlBr}_{3}$-planar & & \\
\hline C $\quad-3.700622559$ & 1.144049525 & 0.616618514 \\
\hline C -2.492464542 & 0.398549348 & 0.668253601 \\
\hline O $\quad-3.942298174$ & 2.196078062 & 1.305537701 \\
\hline
\end{tabular}


$\begin{array}{lllll}\text { O } & -4.637742043 & 0.695193529 & -0.212864876\end{array}$

$\begin{array}{lllll}\text { C } & -5.871695518 & 1.446386337 & -0.286175609\end{array}$

$\begin{array}{llll}\text { C } & -2.230458498 & -0.780069172 & -0.125906289\end{array}$

$\begin{array}{llll}\text { C } & -1.003614783 & -1.438791633 & -0.132614002\end{array}$

$\begin{array}{lllll}\text { C } & -0.836529911 & -2.626576424 & -0.945547462\end{array}$

H $\quad-1.682451725 \quad-2.968708038 \quad-1.535766959$

$\begin{array}{llll}\text { C } & 0.352406085 & -3.309822559 & -0.970535636\end{array}$

$\begin{array}{lllll}\mathrm{H} & 0.453054309 & -4.200016975 & -1.584447265\end{array}$

$\begin{array}{llll}\text { C } & 1.456070185 & -2.863934994 & -0.199036449\end{array}$

H $\quad 2.393101692 \quad-3.411528826 \quad-0.222793177$

C $\quad 1.331045985-1.706660509 \quad 0.595957816$

$\begin{array}{llll}\mathrm{H} & 2.179152012 & -1.363598228 & 1.181893349\end{array}$

$\begin{array}{llll}\text { C } & 0.147743449 & -1.003585219 & 0.638463676\end{array}$

$\begin{array}{lllll}\mathrm{H} & 0.082910925 & -0.111509860 & 1.250821829\end{array}$

$\mathrm{H} \quad-1.755026579 \quad 0.759301186 \quad 1.373989701$

$\begin{array}{lllll}\mathrm{H} & -6.484318733 & 0.916440189 & -1.014808655\end{array}$

$\mathrm{H} \quad-5.670259476 \quad 2.470025063 \quad-0.612389743$

$\begin{array}{lllll}\mathrm{H} & -6.357443810 & 1.468853235 & 0.692793667\end{array}$

H $\quad-3.034170866 \quad-1.156708837 \quad-0.749283314$

$\begin{array}{llll}\text { Al } & -2.889342070 & 3.202149391 & 2.449187279\end{array}$

$\begin{array}{llll}\text { Br } & -1.028478861 & 3.717915535 & 1.127576113\end{array}$

$\begin{array}{llll}\mathrm{Br} & -2.332577467 & 1.713900685 & 4.157998085\end{array}$

$\begin{array}{llll}\mathrm{Br} & -4.215941906 & 4.986497402 & 3.050581217\end{array}$
B $\quad-3.467204571 \quad 3.179549694 \quad 2.444539070$

$\begin{array}{llll}\text { N } & -3.934216499 & 4.640229702 & 1.832710743\end{array}$

$\begin{array}{llll}\text { O } & -2.083651543 & 3.353999138 & 2.665727854\end{array}$

$\begin{array}{llll}\text { C } & -4.265894413 & 2.625465631 & 3.715851545\end{array}$

$\begin{array}{llll}\text { C } & -3.878609657 & 2.972221851 & 5.020051479\end{array}$

$\begin{array}{llll}\text { C } & -4.452976704 & 2.469490767 & 6.179551601\end{array}$

H $\quad-4.104113102 \quad 2.775986671 \quad 7.157419205$

$\begin{array}{llll}\text { C } & -5.498331070 & 1.565503597 & 6.030798435\end{array}$

$\begin{array}{llll}\text { C } & -5.969943523 & 1.181887388 & 4.780901909\end{array}$

$\begin{array}{llll}\mathrm{H} & -6.793438911 & 0.487241507 & 4.675887108\end{array}$

$\begin{array}{llll}\text { C } & -5.344246864 & 1.730665445 & 3.670255661\end{array}$

$\begin{array}{llll}\text { C } & -2.619293928 & 5.409420490 & 1.727800250\end{array}$

$\begin{array}{llll}\text { C } & -4.908072472 & 5.489690781 & 2.634363890\end{array}$

$\begin{array}{llll}\text { C } & -4.153650284 & 6.793035984 & 2.934271812\end{array}$

$\begin{array}{llll}\text { C } & -2.683495760 & 6.355315685 & 2.928466082\end{array}$

$\begin{array}{llll}\mathrm{H} & -2.613452673 & 5.986127377 & 0.801408887\end{array}$

H $\quad-5.149010658 \quad 4.943609715 \quad 3.545271635$

$\mathrm{H} \quad-5.826846600 \quad 5.623104572 \quad 2.060989618$

$\begin{array}{llll}\mathrm{H} & -4.468325615 & 7.227455139 & 3.885934114\end{array}$

$\begin{array}{llll}\mathrm{H} & -4.339086533 & 7.535201073 & 2.150194407\end{array}$

H $\quad-2.437744856 \quad 5.808394909 \quad 3.841875553$

H $\quad-1.976717114 \quad 7.179496288 \quad 2.823556185$

$\begin{array}{llll}\text { C } & -1.265087366 & 3.619228601 & 0.416816294\end{array}$

$\begin{array}{lllll}\text { C } & -1.795427799 & 4.016878605 & -0.809927225\end{array}$

$\begin{array}{lllll}\mathrm{H} & -2.382498980 & 4.927121162 & -0.897504807\end{array}$

$\begin{array}{lllll}\text { C } & -1.583735585 & 3.254285097 & -1.970937848\end{array}$

C $\quad-0.846542180 \quad 2.071524382-1.870322704$

H $\quad-0.690507293 \quad 1.464972854-2.760211468$

$\begin{array}{llll}\text { C } & -0.293569475 & 1.654196501 & -0.650911272\end{array}$

$\begin{array}{llll}\text { C } & -0.498238623 & 2.449541807 & 0.475578189\end{array}$

$\begin{array}{llll}\mathrm{H} & -0.076134913 & 2.150938988 & 1.430037260\end{array}$

$\begin{array}{llll}\text { C } & -1.521903753 & 4.317345142 & 1.761346698\end{array}$

$\begin{array}{llll}\text { C } & -0.218139574 & 4.857722282 & 2.332597971\end{array}$

$\begin{array}{llll}\mathrm{C} & 0.611148179 & 5.635189533 & 1.513452291\end{array}$

$\begin{array}{llll}\mathrm{H} & 0.337120235 & 5.806272984 & 0.475458622\end{array}$

$\begin{array}{llll}\text { C } & 1.801038861 & 6.170068741 & 2.009391785\end{array}$

$\begin{array}{llll}\text { C } & 2.150329351 & 5.900887966 & 3.341586351\end{array}$

$\begin{array}{lllll}\mathrm{H} & 3.080853224 & 6.305371761 & 3.734392643\end{array}$

C $\quad 1.341203451 \quad 5.123281956 \quad 4.172310829$

C $\quad 0.143064529 \quad 4.608176708 \quad 3.654982090$

$\begin{array}{llll}\mathrm{H} & -0.513071656 & 4.016109467 & 4.282996178\end{array}$

$\begin{array}{llll}\mathrm{H} & -4.306204319 & 4.468377113 & 0.899344623\end{array}$

$\begin{array}{llll}\text { C } & 2.699856758 & 7.019686222 & 1.142741203\end{array}$

H $\quad 3.7352385526 .664585590 \quad 1.179577351$

$\begin{array}{llll}\mathrm{H} & 2.377565384 & 7.013149738 & 0.097866312\end{array}$

H $\quad 2.705589771 \quad 8.061058044 \quad 1.485289693$

C $\quad 1.750788569 \quad 4.807704926 \quad 5.592175007$

$\begin{array}{lllll}\mathrm{H} & 0.887357593 & 4.800915241 & 6.265248775\end{array}$

$\begin{array}{lllll}\mathrm{H} & 2.217214823 & 3.816393614 & 5.653017521\end{array}$

$\begin{array}{llll}\mathrm{H} & 2.474333763 & 5.532895565 & 5.974381447\end{array}$

C $-2.161838293 \quad 3.688691616 \quad-3.298278332$

H $\quad-1.403586507 \quad 3.663327694-4.087301254$

H $\quad-2.973641157 \quad 3.023591757 \quad-3.616569519$

$\begin{array}{lllll}\mathrm{H} & -2.563906431 & 4.704650402 & -3.252025843\end{array}$

C $\quad 0.491244733 \quad 0.367531985-0.557976663$

\section{${ }^{1}$ 4-LA}


$\begin{array}{llll}\mathrm{H} & 0.670327663 & 0.079259783 & 0.481379122\end{array}$

$\begin{array}{lllll}\mathrm{H} & -0.040275037 & -0.454679579 & -1.050260305\end{array}$

$\mathrm{H} \quad \begin{array}{llll}\mathrm{H} & 1.464664221 & 0.461622208 & -1.051906943\end{array}$

$\begin{array}{llll}\text { F } & -5.834951401 & 1.347111464 & 2.462128162\end{array}$

F $\quad-6.075588703 \quad 1.056074858 \quad 7.125040054$

F $\quad-2.887910604 \quad 3.879930258 \quad 5.190410614$

$\begin{array}{llll}\text { C } & -3.360836744 & 1.086992502 & 1.047251463\end{array}$

$\begin{array}{llll}\text { C } & -2.461734533 & 0.423400253 & 1.944073915\end{array}$

$\begin{array}{llll}\mathrm{O} & -3.760486603 & 2.290890217 & 1.195808411\end{array}$

$\begin{array}{lllll}\text { O } & -3.784155846 & 0.428232998 & -0.004023033\end{array}$

$\begin{array}{llll}\text { C } & -4.688850403 & 1.100767970 & -0.915901542\end{array}$

$\begin{array}{llll}\text { C } & -2.036782026 & -0.852160215 & 1.750813246\end{array}$

$\begin{array}{llll}\text { C } & -1.041242242 & -1.541837335 & 2.546483278\end{array}$

$\begin{array}{llll}\text { C } & -0.766362071 & -2.894075155 & 2.254813910\end{array}$

$\begin{array}{llll}\mathrm{H} & -1.317141175 & -3.389456987 & 1.459587574\end{array}$

C $\quad 0.195422783 \quad-3.595802546 \quad 2.973999500$

H $\quad 0.395689398 \quad-4.636714458 \quad 2.740916014$

C $\quad 0.905475259-2.954339027 \quad 3.991223574$

H $\quad 1.660749316 \quad-3.497719288 \quad 4.550929070$

$\begin{array}{lllll}\text { C } & 0.648944259 & -1.609855533 & 4.289041519\end{array}$

$\begin{array}{llll}\mathrm{H} & 1.207983851 & -1.114406824 & 5.076532841\end{array}$

C $\quad-0.313089550-0.907041609 \quad 3.578051090$

$\begin{array}{llll}\mathrm{H} & -0.493226916 & 0.136150524 & 3.814126015\end{array}$

$\mathrm{H} \quad-2.087481976 \quad 1.035659194 \quad 2.749475956$

$\begin{array}{lllll}\mathrm{H} & -4.931235313 & 0.355013132 & -1.670614600\end{array}$

H $\quad-4.180697918 \quad 1.958375216 \quad-1.361214995$

$\begin{array}{llll}\mathrm{H} & -5.583357811 & 1.418353915 & -0.377078980\end{array}$

$\begin{array}{llll}\mathrm{H} & -2.455790281 & -1.411231518 & 0.916602552\end{array}$

\begin{tabular}{|c|c|c|c|}
\hline \multicolumn{4}{|c|}{${ }^{3} 4-\mathrm{LA}$} \\
\hline B & -3.597297907 & 3.216954470 & 2.4440026 \\
\hline $\mathrm{N}$ & -3.943701744 & 4.723009586 & 1.84502112 \\
\hline $\mathrm{O}$ & -2.205040932 & 3.319000721 & 911886 \\
\hline $\mathrm{C}$ & -4.438133717 & 2.712686777 & 3.710615158 \\
\hline $\mathrm{C}$ & -4.110421658 & 3.141623735 & 5.005772591 \\
\hline $\mathrm{C}$ & -4.716880798 & 2.698662519 & 6.172063351 \\
\hline $\mathrm{H}$ & -4.413146973 & 3.072211504 & \\
\hline $\mathrm{C}$ & -5.736022472 & 1.762 & \\
\hline $\mathrm{C}$ & -6.1466569 & & \\
\hline $\mathrm{H}$ & -6.9480547 & & 4.704201698 \\
\hline $\mathrm{C}$ & -5.49 & 1.78 & 378 \\
\hline $\mathrm{C}$ & -2.57 & 5.3842 & 4343 \\
\hline $\mathrm{C}$ & -4.836091518 & 5.653103352 & 2.65 \\
\hline $\mathrm{C}$ & -4.006 & 6.926 & 7402 \\
\hline $\mathrm{C}$ & -2.56 & 6.398190 & 2.862303734 \\
\hline $\mathrm{H}$ & -2.515417337 & 5.907300472 & 0.759326518 \\
\hline $\mathrm{H}$ & -5.059173584 & 5.162324905 & 3.596144915 \\
\hline $\mathrm{H}$ & -5.773661137 & & 2.113009691 \\
\hline $\mathrm{H}$ & -4.2792963 & $7.437691 \mathrm{C}$ & 81915 \\
\hline $\mathrm{H}$ & -4.159665585 & 7.627164841 & 2.027029514 \\
\hline $\mathrm{H}$ & -2.349543095 & 5.889252186 & 3.803897142 \\
\hline $\mathrm{H}$ & -1.810588837 & 7.169012547 & 2.708017826 \\
\hline $\mathrm{C}$ & -1.284050226 & 3.463906527 & 0.505348563 \\
\hline $\mathrm{C}$ & -1.891158462 & 3.737527132 & -0.714766979 \\
\hline
\end{tabular}

$\begin{array}{llll}\mathrm{H} & -2.550753117 & 4.592735291 & -0.830926776\end{array}$

C $-1.6840423352 .909778595 \quad-1.837793589$

C $\quad-0.843532503 \quad 1.802092433 \quad-1.713922024$

$\mathrm{H} \quad-0.674663782 \quad 1.162393570 \quad-2.577050686$

$\begin{array}{llll}\text { C } & -0.195037410 & 1.511434436 & -0.507811487\end{array}$

$\begin{array}{llll}\text { C } & -0.438520998 & 2.345380783 & 0.596020222\end{array}$

$\begin{array}{llll}\mathrm{H} & 0.064918131 & 2.144373178 & 1.535926461\end{array}$

C $\quad-1.550530791 \quad 4.218720436 \quad 1.820239544$

$\begin{array}{llll}\text { C } & -0.233626425 & 4.680751801 & 2.434664488\end{array}$

$\begin{array}{llll}\mathrm{C} & 0.689648926 & 5.375258446 & 1.642454147\end{array}$

$\begin{array}{llll}\mathrm{H} & 0.474213719 & 5.550261497 & 0.591140389\end{array}$

$\begin{array}{llll}\text { C } & 1.896208048 & 5.825589657 & 2.181306601\end{array}$

C $\quad 2.165215015 \quad 5.558151722 \quad 3.531552553$

H $\quad 3.106065512 \quad 5.898189545 \quad 3.959056854$

$\begin{array}{llll}\text { C } & 1.261121631 & 4.864255428 & 4.338507175\end{array}$

$\begin{array}{llll}\text { C } & 0.050927043 & 4.431594849 & 3.776084185\end{array}$

$\begin{array}{llll}\mathrm{H} & -0.678337872 & 3.909862757 & 4.385256290\end{array}$

$\begin{array}{llll}\mathrm{H} & -4.344262600 & 4.576323509 & 0.919738472\end{array}$

$\begin{array}{llll}\text { C } & 2.895947456 & 6.584208488 & 1.341269970\end{array}$

$\mathrm{H} \quad 3.895471573 \quad 6.144029140 \quad 1.423132300$

$\begin{array}{llll}\mathrm{H} & 2.614969015 & 6.589768410 & 0.284572393\end{array}$

$\begin{array}{llll}\mathrm{H} & 2.976607561 & 7.626124859 & 1.672096610\end{array}$

C $\quad 1.579984665 \quad 4.553591251 \quad 5.782313347$

H $\quad 0.684155345 \quad 4.605454445 \quad 6.409180641$

$\mathrm{H} \quad 1.987763882 \quad 3.539910555 \quad 5.883617401$

$\begin{array}{llll}\mathrm{H} & 2.321815968 & 5.245663643 & 6.189945221\end{array}$

$\begin{array}{lllll}\text { C } & -2.372246265 & 3.204481840 & -3.148096561\end{array}$

H $\quad-1.663179040 \quad 3.179724455 \quad-3.981774330$

$\mathrm{H} \quad-3.1417658332 .453102589 \quad-3.364337683$

$\begin{array}{lllll}\mathrm{H} & -2.853991032 & 4.186071396 & -3.144338608\end{array}$

$\begin{array}{lllll}\text { C } & 0.768484116 & 0.358383238 & -0.404274106\end{array}$

$\begin{array}{llll}\mathrm{H} & 1.024849892 & 0.137175605 & 0.634035587\end{array}$

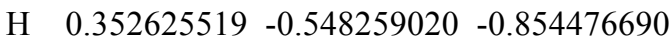

H $\quad 1.699026346 \quad 0.587832928 \quad-0.936941803$

F $\quad-5.923477173 \quad 1.311327100 \quad 2.482988834$

$\begin{array}{llll}\text { F } & -6.346618652 & 1.308380008 & 7.139128685\end{array}$

F $\quad-3.146105051 \quad 4.085185051 \quad 5.153816700$

C $\quad-3.461579561 \quad 1.1864309310 .990412176$

$\begin{array}{llll}\text { C } & -2.463805914 & 0.559323549 & 1.760850668\end{array}$

$\begin{array}{llll}\text { O } & -3.920025110 & 2.384474993 & 1.201321483\end{array}$

$\begin{array}{lllll}\text { O } & -3.950568199 & 0.516034544 & -0.044312835\end{array}$

C $\quad-5.036921501 \quad 1.112204313-0.783038974$

$\begin{array}{llll}\text { C } & -1.951013923 & -0.761861861 & 1.522424102\end{array}$

$\begin{array}{llll}\text { C } & -0.847594261 & -1.277332187 & 2.215424061\end{array}$

$\begin{array}{llll}\text { C } & -0.388419092 & -2.612309694 & 1.928349257\end{array}$

H $\quad-0.928728640 \quad-3.196399689 \quad 1.188956141$

$\begin{array}{llll}\text { C } & 0.703295052 & -3.146248341 & 2.573033094\end{array}$

H $\quad 1.027419209-4.1563625342 .342654228$

C $\quad 1.405344248 \quad-2.389549971 \quad 3.535781622$

H $\quad 2.264276743 \quad-2.816146135 \quad 4.043020725$

C $\quad 0.985245049 \quad-1.079339266 \quad 3.835311651$

H $\quad 1.528226972 \quad-0.498679698 \quad 4.574798584$

$\begin{array}{llll}\text { C } & -0.107166559 & -0.525507689 & 3.201705217\end{array}$

$\begin{array}{llll}\mathrm{H} & -0.405705124 & 0.486693203 & 3.450666189\end{array}$

$\begin{array}{llll}\mathrm{H} & -2.101673126 & 1.143094063 & 2.592118740\end{array}$

H $\quad-5.331278801 \quad 0.360794693 \quad-1.513827801$ 
H $\quad-4.693625927 \quad 2.021025658 \quad-1.283137798$

H $\quad-5.862378597 \quad 1.344525456 \quad-0.106941029$

H $\quad-2.426620007 \quad-1.368830681 \quad 0.759892106$

\begin{tabular}{|c|c|c|c|}
\hline \multicolumn{4}{|c|}{${ }^{1} 4 a-L A$} \\
\hline B & -3.358572483 & 3.252842903 & \\
\hline $\mathrm{N}$ & -3.907421112 & 4.610381603 & 1.774882555 \\
\hline $\mathrm{O}$ & -1.996800303 & 3.537857533 & 2.762310266 \\
\hline $\mathrm{C}$ & -4.187740326 & 2.768274069 & 3.816291332 \\
\hline $\mathrm{C}$ & -3.673358440 & 2.647531271 & 5.112428665 \\
\hline $\mathrm{C}$ & -4.391687393 & 2.154494762 & 6.194987297 \\
\hline $\mathrm{H}$ & -3.942533731 & 2.071493149 & 7.176202774 \\
\hline $\mathrm{C}$ & -5.707371235 & & \\
\hline $\mathrm{C}$ & & & \\
\hline $\mathrm{H}$ & -7.334759235 & 1.562340021 & 4.554047108 \\
\hline $\mathrm{C}$ & -5.520185471 & & \\
\hline $\mathrm{C}$ & -2.6520 & 5.4660 & 92207 \\
\hline $\mathrm{C}$ & & & \\
\hline $\mathrm{C}$ & -4.3129 & 6.78 & 19175 \\
\hline $\mathrm{C}$ & & & \\
\hline $\mathrm{H}$ & & & \\
\hline $\mathrm{H}$ & -5.347550869 & 4.924 & 570032 \\
\hline $\mathrm{H}$ & & & 86778 \\
\hline $\mathrm{H}$ & -4.6959128 & 7.18 & 46922 \\
\hline $\mathrm{H}$ & -4.494684 & & \\
\hline $\mathrm{H}$ & -2.5728 & 5.91 & 0898 \\
\hline $\mathrm{H}$ & -2.15 & & 93550 \\
\hline $\mathrm{C}$ & -1.215 & 3.6 & \\
\hline $\mathrm{C}$ & -1.7164 & & 97681 \\
\hline $\mathrm{H}$ & & & \\
\hline $\mathrm{C}$ & -1.48 & & 3873 \\
\hline $\mathrm{C}$ & -0.758622169 & 2.00 & 70917 \\
\hline $\mathrm{H}$ & -0.59 & 1.3 & 3471 \\
\hline $\mathrm{C}$ & -0.22 & & 90540 \\
\hline $\mathrm{C}$ & -0.454737157 & 2.502223969 & \\
\hline $\mathrm{H}$ & -0.06 & & 62378 \\
\hline $\mathrm{C}$ & & & \\
\hline $\mathrm{C}$ & -0.2172 & & 44033 \\
\hline $\mathrm{C}$ & & & \\
\hline $\mathrm{H}$ & 0.283106953 & & 79196 \\
\hline $\mathrm{C}$ & 1.708000779 & 6.526346684 & 4514 \\
\hline $\mathrm{C}$ & 2.064323902 & 6.401605129 & 3.207283258 \\
\hline $\mathrm{H}$ & 2.958919764 & 6.9066 & 72467 \\
\hline $\mathrm{C}$ & 1.306954384 & & 32800 \\
\hline $\mathrm{C}$ & 0.153060719 & 4.999360561 & 28350 \\
\hline $\mathrm{H}$ & & & \\
\hline $\mathrm{H}$ & -4.198719501 & 4.313387871 & 0.844183266 \\
\hline $\mathrm{C}$ & 2.549654961 & 7.356882572 & 0.917241156 \\
\hline $\mathrm{H}$ & 3.605593681 & 7.072591305 & 0.976387024 \\
\hline $\mathrm{H}$ & 2.227352858 & 7.242151737 & -0.121368237 \\
\hline $\mathrm{H}$ & & 8.421396255 & 1.171590924 \\
\hline $\mathrm{C}$ & 1.730539799 & 5.483181953 & 5.543004036 \\
\hline $\mathrm{H}$ & 0.865011454 & 5.381974220 & 6.205293655 \\
\hline $\mathrm{H}$ & 2.347644329 & 4.585292339 & 5.672522545 \\
\hline
\end{tabular}

H $\quad 2.322163820 \quad 6.337097645 \quad 5.884777069$

C $\quad-1.992513418 \quad 3.561686754 \quad-3.259285212$

$\mathrm{H} \quad-1.131519914 \quad 3.980647087 \quad-3.893560410$

H $\quad-2.408550501 \quad 2.642701626-3.805113316$

H $\quad-2.805335999 \quad 4.355147839 \quad-3.181040287$

C $\quad 0.582835495 \quad 0.387039483 \quad-0.288907915$

H $\quad 0.625277042 \quad 0.075219348 \quad 0.758518577$

H $\quad 0.162343413 \quad-0.435451925 \quad-0.875125885$

H $\quad 1.613452911 \quad 0.534940064 \quad-0.632027268$

F $\quad-6.092943192 \quad 2.477599621 \quad 2.459480524$

F $-6.425044060 \quad 1.287829518 \quad 6.990254402$

F $\quad-2.386294842 \quad 2.995973587 \quad 5.350231171$

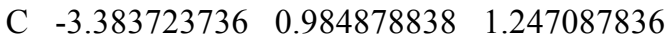

C $-2.721886158 \quad 0.070837736 \quad 2.129855871$

$\begin{array}{llll}\text { O } & -3.574830055 & 2.237658024 & 1.371337652\end{array}$

$\begin{array}{lllll}\text { O } & -3.869602442 & 0.411566049 & 0.166427240\end{array}$

C $\quad-4.573842049 \quad 1.234787703 \quad-0.793907702$

C $\quad-1.958149910 \quad 0.440452307 \quad 3.193442822$

C $\quad-1.277215958 \quad-0.440143108 \quad 4.118536949$

C $\quad-0.627774775 \quad 0.156266809 \quad 5.220630646$

H $\quad-0.676110029 \quad 1.234790444 \quad 5.340259552$

C $\quad 0.036332961 \quad-0.626880229 \quad 6.158475876$

H $\quad 0.528540909 \quad-0.159829319 \quad 7.005558491$

C $\quad 0.064107291 \quad-2.015060425 \quad 6.008457184$

H $\quad 0.581956565 \quad-2.629178524 \quad 6.738972187$

C $\quad-0.573820412 \quad-2.621243238 \quad 4.918375015$

H $\quad-0.547104180 \quad-3.700543404 \quad 4.806615829$

C $\quad-1.239512205-1.844640374 \quad 3.980673552$

H $\quad-1.726096869-2.326630354 \quad 3.138693333$

H $-2.854134560 \quad-0.967697203 \quad 1.852878809$

$\mathrm{H} \quad-4.906795502 \quad 0.542577624 \quad-1.565054178$

H $\quad-3.888810396 \quad 1.979322314 \quad-1.205569148$

H $\quad-5.425297737 \quad 1.718078613 \quad-0.309413522$

H $\quad-1.807652116 \quad 1.497042298 \quad 3.379520178$

\begin{tabular}{|c|c|c|c|}
\hline \multicolumn{4}{|c|}{${ }^{3} 4 a-L A$} \\
\hline B & -3.615216017 & 3.288698196 & 2.529475689 \\
\hline $\mathrm{N}$ & -4.001518250 & 4.715983391 & 1.758162498 \\
\hline $\mathrm{O}$ & -2.236586571 & 3.458811283 & 2.804308653 \\
\hline $\mathrm{C}$ & -4.494219303 & 2.919488907 & 3.814855337 \\
\hline $\mathrm{C}$ & -4.165588856 & 3.400553465 & 5.091329575 \\
\hline $\mathrm{C}$ & -4.825330257 & 3.058896542 & 6.263716698 \\
\hline $\mathrm{H}$ & -4.521147728 & 3.465935469 & 7.219429970 \\
\hline $\mathrm{C}$ & -5.900478363 & 2.184458733 & 6.154677868 \\
\hline $\mathrm{C}$ & -6.315799236 & 1.673416615 & 4.931242943 \\
\hline $\mathrm{H}$ & -7.161074638 & 1.001372337 & 4.856573105 \\
\hline $\mathrm{C}$ & -5.604210377 & 2.062664270 & 3.804173946 \\
\hline $\mathrm{C}$ & -2.656410933 & 5.423278332 & 1.636264563 \\
\hline $\mathrm{C}$ & -4.976360321 & 5.676469326 & 2.417739153 \\
\hline $\mathrm{C}$ & -4.188850403 & 6.980435371 & 2.610550165 \\
\hline $\mathrm{C}$ & -2.737319708 & 6.497167587 & 2.723354101 \\
\hline $\mathrm{H}$ & -2.583794832 & 5.896982670 & 0.655862749 \\
\hline $\mathrm{H}$ & -5.278000355 & 5.239719868 & 3.369283915 \\
\hline $\mathrm{H}$ & -5.863632679 & 5.777009964 & 1.790322542 \\
\hline
\end{tabular}


H $\quad-4.528484344 \quad 7.527247906 \quad 3.493065119$

$\begin{array}{llll}\mathrm{H} & -4.311202526 & 7.637433052 & 1.742383003\end{array}$

$\mathrm{H} \quad-2.555034161 \quad 6.045355320 \quad 3.701178312$

$\begin{array}{llll}\mathrm{H} & -1.995824099 & 7.281449318 & 2.565344095\end{array}$

$\begin{array}{llll}\text { C } & -1.290167928 & 3.461678743 & 0.598481238\end{array}$

C $\quad-1.757524729 \quad 3.715766668 \quad-0.684707582$

$\begin{array}{lllll}\mathrm{H} & -2.360540152 & 4.593924999 & -0.897904098\end{array}$

$\begin{array}{llll}\text { C } & -1.453517437 & 2.846854687 & -1.756251693\end{array}$

$\begin{array}{llll}\text { C } & -0.672417104 & 1.717506528 & -1.508169532\end{array}$

$\begin{array}{llll}\mathrm{H} & -0.429369181 & 1.047747254 & -2.329817772\end{array}$

$\begin{array}{llll}\text { C } & -0.179406688 & 1.438656688 & -0.226599440\end{array}$

$\begin{array}{llll}\text { C } & -0.499603748 & 2.317822933 & 0.817035079\end{array}$

$\begin{array}{llll}\mathrm{H} & -0.110898517 & 2.137309551 & 1.813255429\end{array}$

$\begin{array}{llll}\text { C } & -1.601475358 & 4.305113792 & 1.845888495\end{array}$

$\begin{array}{llll}\text { C } & -0.307681650 & 4.859370232 & 2.432465315\end{array}$

C $\quad 0.597582757 \quad 5.525768280 \quad 1.596390605$

$\begin{array}{llll}\mathrm{H} & 0.391702056 & 5.604087353 & 0.531618059\end{array}$

$\begin{array}{llll}\text { C } & 1.773330212 & 6.074774742 & 2.111631155\end{array}$

$\begin{array}{llll}\text { C } & 2.029923677 & 5.933504105 & 3.483095407\end{array}$

$\begin{array}{llll}\mathrm{H} & 2.946129322 & 6.352524281 & 3.893808603\end{array}$

C $\quad 1.144542456 \quad 5.266048908 \quad 4.332813740$

C $\quad-0.034636386 \quad 4.732063293 \quad 3.793173313$

$\begin{array}{llll}\mathrm{H} & -0.747784913 & 4.225136280 & 4.433088303\end{array}$

$\begin{array}{lllll}\mathrm{H} & -4.340915203 & 4.456436634 & 0.833304644\end{array}$

$\begin{array}{llll}\text { C } & 2.750966311 & 6.808897018 & 1.224829435\end{array}$

$\begin{array}{lllll}\mathrm{H} & 3.769210815 & 6.428467751 & 1.358727217\end{array}$

$\begin{array}{llll}\mathrm{H} & 2.490402937 & 6.710712910 & 0.167399436\end{array}$

$\begin{array}{llll}\mathrm{H} & 2.773727179 & 7.877950191 & 1.466273546\end{array}$

C $\quad 1.452330470 \quad 5.091303349 \quad 5.801354885$

$\begin{array}{lllll}\mathrm{H} & 0.543723822 & 5.151279449 & 6.408925056\end{array}$

$\begin{array}{lllll}\mathrm{H} & 1.905706882 & 4.110342979 & 5.990843773\end{array}$

$\begin{array}{lllll}\mathrm{H} & 2.152913570 & 5.850152493 & 6.160301208\end{array}$

$\begin{array}{llll}\text { C } & -2.014541149 & 3.115208149 & -3.130820274\end{array}$

H $-1.366915703 \quad 2.481986523 \quad-3.968835115$

$\begin{array}{lllll}\mathrm{H} & -3.199174643 & 2.728813410 & -3.156933784\end{array}$

$\begin{array}{lllll}\mathrm{H} & -1.966871500 & 4.314968109 & -3.346552372\end{array}$

$\begin{array}{llll}\text { C } & 0.701386034 & 0.239052683 & 0.016112655\end{array}$

$\begin{array}{lllll}\mathrm{H} & 0.823640168 & 0.036523309 & 1.083104491\end{array}$

$\begin{array}{lllll}\mathrm{H} & 0.292287886 & -0.657247841 & -0.462992758\end{array}$

$\begin{array}{lllll}\mathrm{H} & 1.699183702 & 0.400368124 & -0.408123881\end{array}$

F $\quad-6.038013458 \quad 1.559823513 \quad 2.622934580$

F $\quad-6.561979294 \quad 1.831042767 \quad 7.262940407$

F $\quad-3.141867638 \quad 4.281089783 \quad 5.217386246$

$\begin{array}{llll}\text { C } & -3.408780336 & 1.151413321 & 1.123020768\end{array}$

$\begin{array}{llll}\text { C } & -2.520417929 & 0.340510756 & 1.867469072\end{array}$

$\begin{array}{llll}\text { O } & -3.888041973 & 2.323828697 & 1.378425121\end{array}$

$\begin{array}{lllll}\text { O } & -3.792877674 & 0.598267853 & -0.028795490\end{array}$

$\begin{array}{llll}\text { C } & -4.747221947 & 1.308021784 & -0.839124262\end{array}$

$\begin{array}{llll}\text { C } & -2.034748077 & 0.525305331 & 3.209319115\end{array}$

$\begin{array}{llll}\text { C } & -1.083312511 & -0.304514319 & 3.818774939\end{array}$

C $\quad-0.670963228 \quad-0.009501505 \quad 5.168892384$

$\begin{array}{llll}\mathrm{H} & -1.116487980 & 0.846821368 & 5.667244434\end{array}$

$\begin{array}{llll}\text { C } & 0.260197431 & -0.785407782 & 5.817572117\end{array}$

$\begin{array}{lllll}\mathrm{H} & 0.551428914 & -0.543656826 & 6.835042953\end{array}$

$\begin{array}{llll}\text { C } & 0.842804432 & -1.898661137 & 5.170117855\end{array}$

H $\quad 1.575308323 \quad-2.508920193 \quad 5.687462807$ $\begin{array}{llll}\text { C } & 0.463686794 & -2.212002754 & 3.851016760\end{array}$

H $\quad 0.908964813 \quad-3.068645477 \quad 3.354371309$

C $-0.469569653-1.445762753 \quad 3.183776379$

$\mathrm{H} \quad-0.744932353 \quad-1.712044358 \quad 2.170320988$

H $\quad-2.258454323 \quad-0.565206110 \quad 1.332867742$

$\mathrm{H} \quad-4.953610897 \quad 0.646565497 \quad-1.679428577$

H $\quad-4.316724777 \quad 2.249474287 \quad-1.189353228$

H $\quad-5.657814026 \quad 1.502109408 \quad-0.266674876$

H $\quad-2.401804447 \quad 1.361818194 \quad 3.782387972$

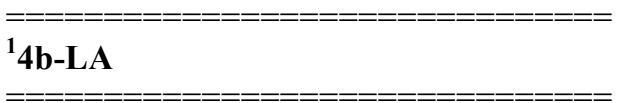

$\begin{array}{llll}\text { B } & -3.463137388 & 3.512163639 & 1.733812690\end{array}$

$\begin{array}{llll}\mathrm{N} & -3.765840292 & 5.042730331 & 1.193235636\end{array}$

$\begin{array}{llll}\text { O } & -2.059148312 & 3.502127171 & 1.913006902\end{array}$

$\begin{array}{llll}\text { C } & -4.362402439 & 3.044858932 & 2.968575001\end{array}$

$\begin{array}{llll}\text { C } & -3.873387098 & 2.808492422 & 4.259841442\end{array}$

$\begin{array}{lllll}\text { C } & -4.641970158 & 2.329747677 & 5.314783096\end{array}$

$\begin{array}{llll}\mathrm{H} & -4.207509518 & 2.157761812 & 6.291199207\end{array}$

$\begin{array}{llll}\text { C } & -5.986166000 & 2.077343225 & 5.066478729\end{array}$

$\begin{array}{llll}\text { C } & -6.565042973 & 2.301187754 & 3.821219206\end{array}$

$\begin{array}{llll}\mathrm{H} & -7.615359783 & 2.112851143 & 3.639978170\end{array}$

$\begin{array}{llll}\text { C } & -5.729773521 & 2.777778625 & 2.822769165\end{array}$

$\begin{array}{llll}\text { C } & -2.463703871 & 5.741522789 & 1.494934320\end{array}$

$\begin{array}{llll}\text { C } & -4.840106010 & 5.880197048 & 1.820412278\end{array}$

$\begin{array}{llll}\text { C } & -4.203208923 & 6.396765232 & 3.124880075\end{array}$

$\begin{array}{llll}\text { C } & -2.670181274 & 6.210741997 & 2.947649717\end{array}$

$\begin{array}{llll}\mathrm{H} & -2.330173969 & 6.587279320 & 0.818191171\end{array}$

$\mathrm{H} \quad-5.740629673 \quad 5.282610416 \quad 1.947189569$

$\begin{array}{llll}\mathrm{H} & -5.060446262 & 6.698642254 & 1.129134774\end{array}$

H $\quad-4.560828209 \quad 5.819041729 \quad 3.979489326$

H $\quad-4.481476784 \quad 7.438841820 \quad 3.294812202$

$\begin{array}{llll}\mathrm{H} & -2.299596548 & 5.443489552 & 3.629429102\end{array}$

H $\quad-2.101587296 \quad 7.121073246 \quad 3.140096426$

$\begin{array}{llll}\text { C } & -1.099988580 & 4.288295269 & -0.165735155\end{array}$

$\begin{array}{lllll}\text { C } & -1.584886432 & 4.997889996 & -1.267988086\end{array}$

$\mathrm{H} \quad-2.197534800 \quad 5.886215687 \quad-1.136064887$

C $-1.277183175 \quad 4.601315022-2.579929590$

$\begin{array}{lllll}\text { C } & -0.476223946 & 3.474185944 & -2.765378475\end{array}$

H $\quad-0.250706732 \quad 3.145871401 \quad-3.776519537$

$\begin{array}{lllll}\text { C } & 0.022396501 & 2.741451979 & -1.680144310\end{array}$

$\begin{array}{lrrrr}\text { C } & -0.296044946 & 3.162908077 & -0.389134794\end{array}$

$\begin{array}{llll}\mathrm{H} & 0.078607805 & 2.610537767 & 0.467207640\end{array}$

$\begin{array}{llll}\text { C } & -1.407510996 & 4.631606102 & 1.302531242\end{array}$

$\begin{array}{llll}\text { C } & -0.120268248 & 4.968134880 & 2.042488337\end{array}$

$\begin{array}{llll}\text { C } & 0.694918692 & 5.988667965 & 1.536062002\end{array}$

H $\quad 0.4260300996 .482893944 \quad 0.605954826$

$\begin{array}{llll}\text { C } & 1.861076951 & 6.367387772 & 2.200575352\end{array}$

$\begin{array}{llll}\text { C } & 2.205721140 & 5.691295624 & 3.380435228\end{array}$

$\begin{array}{llll}\mathrm{H} & 3.115372419 & 5.975957870 & 3.904829025\end{array}$

C $\quad 1.415243506 \quad 4.662863731 \quad 3.895908117$

$\begin{array}{llll}\text { C } & 0.238272488 & 4.310673237 & 3.216797113\end{array}$

H $\quad-0.404572487 \quad 3.532453775 \quad 3.610235929$

H $\quad-3.910352945 \quad 4.963061810 \quad 0.185544491$

C $\quad 2.739611149 \quad 7.476096630 \quad 1.671554565$ 
$\begin{array}{llll}\mathrm{H} & 3.762515068 & 7.122346401 & 1.502313852\end{array}$

$\begin{array}{llll}\mathrm{H} & 2.361321211 & 7.875608921 & 0.726476014\end{array}$

$\begin{array}{llll}\mathrm{H} & 2.801146269 & 8.304507256 & 2.386409521\end{array}$

C $\quad 1.820791841 \quad 3.919199228 \quad 5.146951199$

$\begin{array}{lllll}\mathrm{H} & 0.946382344 & 3.596418858 & 5.720647812\end{array}$

$\mathrm{H} \quad 2.396913052 \quad 3.019428015 \quad 4.897292137$

H $\quad 2.446506023 \quad 4.536684036 \quad 5.797464848$

C $\quad-1.793030500 \quad 5.380576134-3.766863823$

$\mathrm{H} \quad-1.051771045 \quad 6.112504005 \quad-4.109326839$

$\begin{array}{lllll}\mathrm{H} & -2.007874727 & 4.717546940 & -4.610761166\end{array}$

H $\quad-2.706773520 \quad 5.932301998 \quad-3.524608135$

C $\quad 0.878256321 \quad 1.518603086-1.908638954$

$\begin{array}{lllll}\mathrm{H} & 0.784530699 & 0.805494130 & -1.084063172\end{array}$

$\mathrm{H} \quad 0.602606177 \quad 1.009815693 \quad-2.837808847$

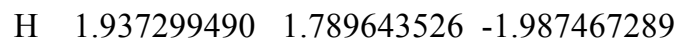

F $\quad-6.294901371 \quad 3.027041197 \quad 1.612268209$

F $\quad-6.751733780 \quad 1.610821128 \quad 6.059464931$

F $\quad-2.570120573 \quad 3.049238205 \quad 4.529233456$

$\begin{array}{llll}\text { C } & -3.268643379 & 1.788723826 & -0.197487041\end{array}$

$\begin{array}{llll}\text { C } & -3.361723900 & 1.814018488 & -1.628742933\end{array}$

$\begin{array}{llll}\text { O } & -3.874338627 & 2.702314377 & 0.457275957\end{array}$

$\begin{array}{llll}\text { O } & -2.589365005 & 0.808757305 & 0.341864169\end{array}$

$\begin{array}{llll}\text { C } & -2.598203897 & 0.534030378 & 1.769083500\end{array}$

$\begin{array}{llll}\text { C } & -2.751700163 & 0.896152198 & -2.419951200\end{array}$

C $\quad-2.6807181840 .904316366-3.866261005$

C $-1.928850293-0.105129756-4.501195908$

H $\quad-1.450006843 \quad-0.870790184 \quad-3.896747589$

C $-1.792165875-0.126691893 \quad-5.885701180$

$\begin{array}{llll}\mathrm{H} & -1.209023833 & -0.908330917 & -6.361855030\end{array}$

$\begin{array}{lllll}\text { C } & -2.409556866 & 0.858558357 & -6.658580780\end{array}$

$\begin{array}{lllll}\mathrm{H} & -2.306500912 & 0.843949318 & -7.739305496\end{array}$

$\begin{array}{lllll}\text { C } & -3.164273977 & 1.866332531 & -6.043618679\end{array}$

$\begin{array}{lllll}\mathrm{H} & -3.646081448 & 2.627936363 & -6.648756504\end{array}$

C $\quad-3.298949480 \quad 1.892287016 \quad-4.662644863$

$\begin{array}{lllll}\mathrm{H} & -3.882273674 & 2.679930687 & -4.198934078\end{array}$

$\begin{array}{lllll}\mathrm{H} & -3.886903524 & 2.671708822 & -2.025875807\end{array}$

$\begin{array}{llll}\mathrm{H} & -2.195276022 & -0.473446608 & 1.855506301\end{array}$

$\mathrm{H} \quad-3.621017218 \quad 0.574949622 \quad 2.146600246$

$\begin{array}{llll}\mathrm{H} & -1.969027519 & 1.258970499 & 2.283663034\end{array}$

$\begin{array}{lllll}\mathrm{H} & -2.226269245 & 0.082610562 & -1.924669623\end{array}$

\begin{tabular}{|c|c|c|c|}
\hline \multicolumn{4}{|c|}{${ }^{3} 4 \mathrm{~b}-\mathrm{LA}$} \\
\hline B & -3.560047865 & 3.374847174 & 1.73457229 \\
\hline $\mathrm{N}$ & -3.910407543 & 4.898377895 & 1.15174758 \\
\hline $\mathrm{O}$ & -2.132959604 & 3.397168398 & 551436 \\
\hline $\mathrm{C}$ & -4.354772568 & 3.013890505 & 8072 \\
\hline $\mathrm{C}$ & -3.776740074 & 2.881090879 & 4.347051620 \\
\hline $\mathrm{C}$ & -4.469237804 & 2.516595840 & 5.496447563 \\
\hline $\mathrm{H}$ & -3.963838577 & 2.423762798 & 6.449176788 \\
\hline $\mathrm{C}$ & -5.831932068 & 2.271407604 & 5.372729301 \\
\hline $\mathrm{C}$ & -6.497155190 & 2.391170025 & 4.157103539 \\
\hline $\mathrm{H}$ & -7.560745239 & 2.210502148 & 4.069618225 \\
\hline $\mathrm{C}$ & -5.731359482 & 2.758652449 & 3.061033487 \\
\hline $\mathrm{C}$ & -2.612684011 & 5.624763012 & 1.37178587 \\
\hline
\end{tabular}

$\begin{array}{llll}\text { C } & -4.967132568 & 5.754824638 & 1.785385251\end{array}$

$\begin{array}{llll}\text { C } & -4.285490990 & 6.337958336 & 3.038255453\end{array}$

$\begin{array}{llll}\text { C } & -2.759835958 & 6.144353867 & 2.815013170\end{array}$

$\begin{array}{lllll}\mathrm{H} & -2.526273966 & 6.453950405 & 0.667033792\end{array}$

$\mathrm{H} \quad-5.855893612 \quad 5.157286644 \quad 1.975782633$

$\begin{array}{llll}\mathrm{H} & -5.223435879 & 6.541322708 & 1.069110632\end{array}$

$\mathrm{H} \quad-4.609986782 \quad 5.803900242 \quad 3.933074713$

$\begin{array}{llll}\mathrm{H} & -4.559044361 & 7.387553692 & 3.165197849\end{array}$

$\mathrm{H} \quad-2.362598181 \quad 5.402527332 \quad 3.509570122$

H $\quad-2.185444355 \quad 7.061345577 \quad 2.950810671$

$\begin{array}{llll}\text { C } & -1.245886564 & 4.192170143 & -0.303919792\end{array}$

$\begin{array}{lllll}\text { C } & -1.512785673 & 5.027438641 & -1.385415077\end{array}$

H $\quad-2.031512737 \quad 5.971768379-1.248583436$

C $-1.084911942 \quad 4.689369678-2.685916901$

$\begin{array}{lllll}\text { C } & -0.373454005 & 3.499541759 & -2.879390717\end{array}$

H $\quad-0.038972035 \quad 3.239471912-3.879804134$

C $\quad-0.1089292692 .628037214-1.819556236$

$\begin{array}{llll}\text { C } & -0.571299255 & 2.974843979 & -0.542386949\end{array}$

$\begin{array}{lllll}\mathrm{H} & -0.380164653 & 2.317580223 & 0.299504846\end{array}$

C $\quad-1.544836640 \quad 4.529367924 \quad 1.168269873$

$\begin{array}{llll}\text { C } & -0.234506905 & 4.913609982 & 1.847400784\end{array}$

$\begin{array}{llll}\text { C } & 0.507983029 & 5.986522198 & 1.333804965\end{array}$

$\begin{array}{llll}\mathrm{H} & 0.176210150 & 6.491170406 & 0.429653168\end{array}$

$\begin{array}{llll}\text { C } & 1.676181793 & 6.411816597 & 1.965560079\end{array}$

$\begin{array}{llll}\text { C } & 2.095029593 & 5.732982159 & 3.120092630\end{array}$

H $\quad 3.003617048 \quad 6.058314323 \quad 3.622113466$

C $\quad 1.376389742 \quad 4.654579639 \quad 3.641913414$

$\begin{array}{lllll}\text { C } & 0.200594649 & 4.251336098 & 2.992819786\end{array}$

$\begin{array}{llll}\mathrm{H} & -0.390201777 & 3.436503887 & 3.390667677\end{array}$

$\begin{array}{llll}\mathrm{H} & -4.097454071 & 4.772266388 & 0.155998439\end{array}$

$\begin{array}{llll}\text { C } & 2.476279974 & 7.577231884 & 1.434326291\end{array}$

H $\quad 3.509959698 \quad 7.282577991 \quad 1.222896576$

$\begin{array}{llll}\mathrm{H} & 2.045120001 & 7.977349758 & 0.512423277\end{array}$

$\begin{array}{llll}\mathrm{H} & 2.518571138 & 8.391313553 & 2.166800261\end{array}$

C $\quad 1.852786660 \quad 3.919032574 \quad 4.872098923$

$\mathrm{H} \quad 1.010465264 \quad 3.546841860 \quad 5.463500500$

H $\quad 2.4657137393 .051972628 \quad 4.596292019$

$\mathrm{H} \quad 2.463562965 \quad 4.560256958 \quad 5.513681412$

C $\quad-1.358892679 \quad 5.610949516 \quad-3.846808672$

$\mathrm{H} \quad-0.518916368 \quad 6.300038338 \quad-4.000088692$

$\mathrm{H} \quad-1.490415215 \quad 5.047313213 \quad-4.775129795$

$\begin{array}{llll}\mathrm{H} & -2.252927780 & 6.219378948 & -3.680983782\end{array}$

C $\quad 0.666812062 \quad 1.354813933-2.037819624$

$\begin{array}{lllll}\mathrm{H} & 0.430030614 & 0.608001411 & -1.275085926\end{array}$

H $\quad 0.452604353 \quad 0.925126135 \quad-3.020953178$

H $\quad 1.744976759 \quad 1.547825813 \quad-1.989655375$

$\begin{array}{llll}\mathrm{F} & -6.385520458 & 2.909521580 & 1.880151391\end{array}$

F $\quad-6.528569698 \quad 1.915103436 \quad 6.458781242$

$\begin{array}{lllll}\text { F } & -2.451481104 & 3.113090992 & 4.496942520\end{array}$

$\begin{array}{llll}\text { C } & -3.341038942 & 1.609360695 & -0.074911892\end{array}$

$\begin{array}{llll}\text { C } & -3.207415104 & 1.735698938 & -1.468364120\end{array}$

$\begin{array}{llll}\mathrm{O} & -4.034158707 & 2.492681503 & 0.585308611\end{array}$

$\begin{array}{llll}\text { O } & -2.781514883 & 0.548766673 & 0.492893696\end{array}$

$\begin{array}{llll}\text { C } & -2.943887472 & 0.300018519 & 1.906241894\end{array}$

$\begin{array}{llll}\text { C } & -2.627475739 & 0.763097167 & -2.341147661\end{array}$

$\begin{array}{llll}\text { C } & -2.482643127 & 0.950460255 & -3.724643230\end{array}$ 
C $-1.834633589-0.064592779-4.511662006$

H $-1.496922612 \quad-0.967931271 \quad-4.012907982$

$\begin{array}{lllll}\text { C } & -1.639860868 & 0.096259348 & -5.864575863\end{array}$

H $-1.144276142 \quad-0.682533085-6.435715675$

C $\quad-2.082953930 \quad 1.267905712-6.518112183$

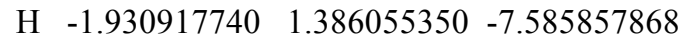

C $\quad-2.729425430 \quad 2.274183035 \quad-5.779700279$

H $\quad-3.080418348 \quad 3.169469357 \quad-6.283945084$

C $\quad-2.928506851 \quad 2.132774353 \quad-4.421026230$

$\mathrm{H} \quad-3.428789616 \quad 2.923537493 \quad-3.875966311$

H $\quad-3.602504253 \quad 2.661439419-1.863177776$

H $\quad-2.593705654 \quad-0.720115364 \quad 2.057283163$

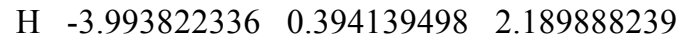

H $\quad-2.335875273 \quad 1.005248427 \quad 2.476327896$

H $\quad-2.260338783-0.155295253 \quad-1.896667957$
$\begin{array}{llll}\mathrm{H} & 3.155002117 & 5.947666168 & 3.749889851\end{array}$

C $\quad 1.419053078 \quad 4.683770180 \quad 3.827330589$

C $\quad 0.215664700 \quad 4.344696045 \quad 3.190091610$

H $\quad-0.433309495 \quad 3.592130899 \quad 3.620443821$

H $\quad-3.980278969 \quad 4.992342949 \quad 0.253448904$

C $\quad 2.756703615 \quad 7.393082619 \quad 1.485418081$

H $\quad 3.767938614 \quad 7.008602142 \quad 1.313015938$

H $\quad 2.371403456 \quad 7.765197277 \quad 0.532051206$

H $\quad 2.849972486 \quad 8.246488571 \quad 2.166760206$

C $\quad 1.839052677 \quad 3.963997126 \quad 5.087502956$

$\mathrm{H} \quad 0.972925305 \quad 3.686878204 \quad 5.696606159$

H $2.378065586 \quad 3.038901329 \quad 4.847781658$

H $\quad 2.503791094 \quad 4.579390526 \quad 5.700184345$

C $\quad-1.867215753 \quad 5.611657619 \quad-3.713612318$

H $\quad-1.080326319 \quad 5.733508587 \quad-4.463652134$

H $\quad-2.722764730 \quad 5.146542549 \quad-4.217999935$

$\mathrm{H} \quad-2.172813177 \quad 6.607882023 \quad-3.381059885$

C $\quad 0.457178950 \quad 1.449311972 \quad-2.026988983$

H $\quad 0.099177338 \quad 0.648952842 \quad-1.370659113$

H $\quad 0.335679650 \quad 1.124286175 \quad-3.064201832$

H $\quad \begin{array}{lllll}1.531045556 & 1.562607646 & -1.841240287\end{array}$

F $\quad-6.339200974 \quad 3.046515703 \quad 1.712897182$

F $-6.744722843 \quad 1.700819731 \quad 6.186306000$

F $-2.564149380 \quad 3.035757303 \quad 4.561293602$

C $\quad-3.328047514 \quad 1.751613975 \quad-0.100568227$

C $\quad-3.366394997 \quad 1.641682744 \quad-1.531713128$

$\begin{array}{lllll}\mathrm{O} & -3.930062532 & 2.697994709 & 0.511203647\end{array}$

$\begin{array}{lllll}\text { O } & -2.671357632 & 0.776587486 & 0.477041602\end{array}$

C $\quad-2.666435003 \quad 0.544869542 \quad 1.910196900$

C $\quad-4.006642342 \quad 2.548121452 \quad-2.310619593$

C $-4.060522556 \quad 2.555280209 \quad-3.759882450$

C $\quad-4.924350262 \quad 3.467236280 \quad-4.399425507$

H $\quad-5.536278725 \quad 4.132834911 \quad-3.796215773$

C $\quad-5.009129047 \quad 3.516414881 \quad-5.788023949$

H $\quad-5.684148788 \quad 4.219587326 \quad-6.265256405$

C $\quad-4.223011017 \quad 2.661276340 \quad-6.563293457$

H $\quad-4.283588886 \quad 2.699514151 \quad-7.646427155$

C $\quad-3.351864338 \quad 1.756696463 \quad-5.943149090$

H $\quad-2.735427141 \quad 1.097504854-6.546350956$

C $\quad-3.270087719 \quad 1.701701880 \quad-4.558262825$

H $\quad-2.578732967 \quad 1.007797837 \quad-4.092888832$

H $\quad-2.840326309 \quad 0.789323568 \quad-1.940745234$

$\begin{array}{llll}\mathrm{H} & -2.259557247 & -0.458586097 & 2.020535707\end{array}$

$\mathrm{H} \quad-3.686155558 \quad 0.592421353 \quad 2.293782473$

H $-2.033962250 \quad 1.283909321 \quad 2.400429964$

H $\quad-4.546718597 \quad 3.347082853 \quad-1.807068110$ 


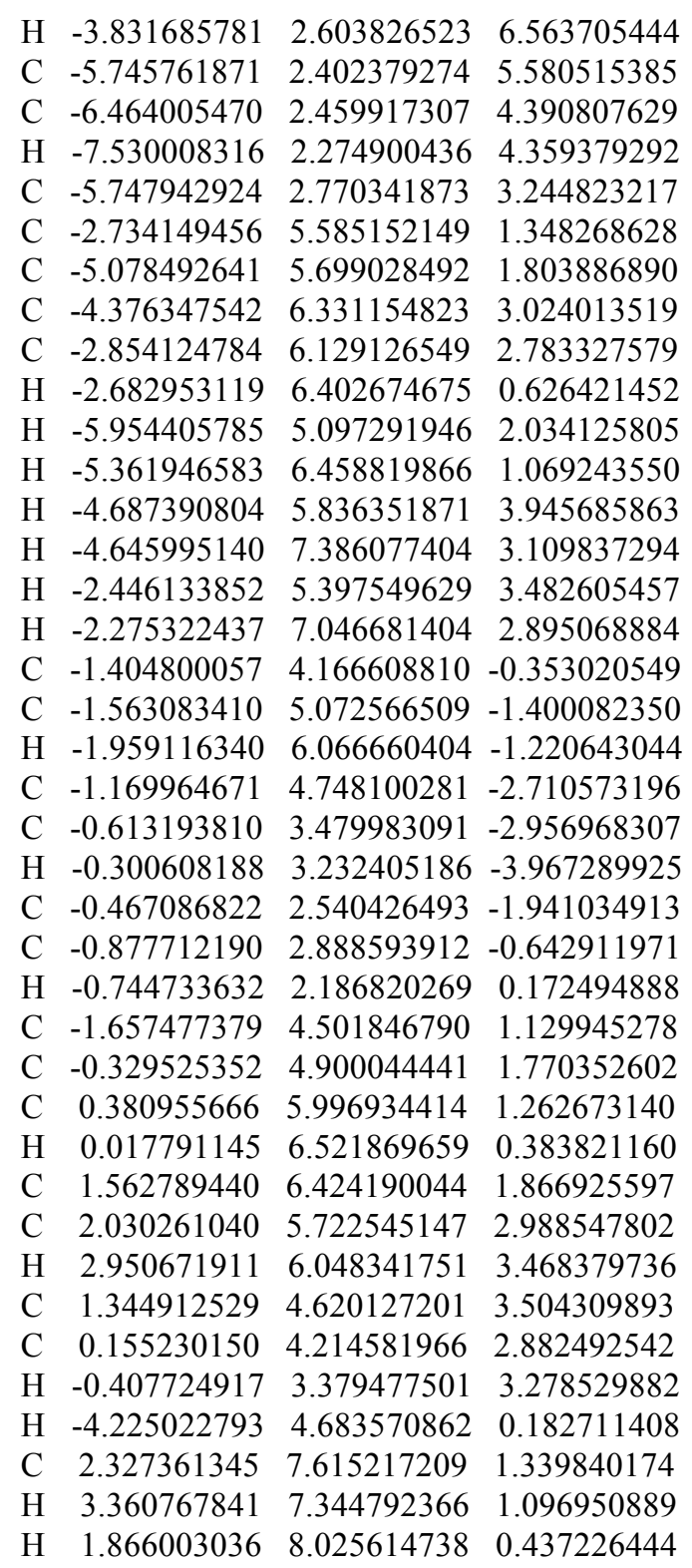

H $\quad 2.372776747 \quad 8.415281296 \quad 2.087470531$

C $\quad 1.871802688 \quad 3.859995365 \quad 4.698554516$

H $\quad 1.054366350 \quad 3.464159966 \quad 5.309172153$

H $\quad 2.483755112 \quad 3.007024527 \quad 4.379995346$

H $\quad 2.497730970 \quad 4.492352009 \quad 5.334325790$

C $\quad-1.329211473 \quad 5.728533268$-3.841150284

H $\quad-0.391154021 \quad 5.842478752 \quad-4.394540787$

$\mathrm{H} \quad-2.081923008 \quad 5.369026661 \quad-4.553328991$

$\mathrm{H} \quad-1.639849663 \quad 6.715031147 \quad-3.487522364$

C $\quad 0.124643549 \quad 1.178544164 \quad-2.203862667$

H $\quad-0.360174656 \quad 0.410136700 \quad-1.592987895$

H $\quad 0.034648046 \quad 0.896283627 \quad-3.256563902$

H $\quad 1.190434217 \quad 1.163982630 \quad-1.950717211$

F $\quad-6.455017567 \quad 2.859584570 \quad 2.088010311$

F $\quad-6.393575668 \quad 2.104127407 \quad 6.713243008$

F $\quad-2.407519579 \quad 3.188675165 \quad 4.512916088$

C $\quad-3.458971739 \quad 1.504707694 \quad 0.064208172$

C $-3.380677223 \quad 1.546714783 \quad-1.335585713$

$\begin{array}{llll}\text { O } & -4.165202141 & 2.381548882 & 0.724465013\end{array}$

$\begin{array}{llll}\text { O } & -2.784167528 & 0.523806632 & 0.657338321\end{array}$

C $\quad-2.892409801 \quad 0.314103007 \quad 2.081151724$

C $-4.038630486 \quad 2.550463200 \quad-2.111947060$

C $\quad-3.875333071 \quad 2.742651463 \quad-3.491369724$

C $\quad-4.574259758 \quad 3.815716743 \quad-4.144303322$

H $\quad-5.223856449 \quad 4.450857639 \quad-3.548528433$

C $\quad-4.436484337 \quad 4.040342808 \quad-5.494636536$

H $\quad-4.978678703 \quad 4.854409218 \quad-5.966027737$

C $\quad-3.597095966 \quad 3.214173794 \quad-6.277761936$

H $\quad-3.493940830 \quad 3.397082090 \quad-7.342189312$

C $\quad-2.908446550 \quad 2.150281668 \quad-5.671716213$

H $\quad-2.272560120 \quad 1.507927060 \quad-6.273299217$

C $\quad-3.033427000 \quad 1.912703633 \quad-4.317009926$

H $-2.491106033 \quad 1.088736653 \quad-3.868555546$

H $-2.822498083 \quad 0.746766806 \quad-1.804402709$

H $-2.498877525 \quad-0.687107801 \quad 2.252532721$

$\mathrm{H} \quad-3.935064077 \quad 0.376789063 \quad 2.397259712$

H $\quad-2.295790672 \quad 1.058812618 \quad 2.611704588$

H $\quad-4.712348938 \quad 3.216453075 \quad-1.582560062$ 
Table S9. Vibrational frequencies (in $\mathrm{cm}^{-1}$ ) of the optimized geometries

\begin{tabular}{|c|c|c|c|c|c|}
\hline \multicolumn{6}{|l|}{$6 f$} \\
\hline 23.03 & 38.03 & 41.95 & 43.73 & 45.48 & .07 \\
\hline 49.58 & 62.65 & $67.49 \quad 7$ & $72.81 \quad 89$ & 39.22102 & 2.21 \\
\hline 105.91 & 122.97 & 151.91 & 176.83 & $184.54 \quad 1$ & 190.52 \\
\hline 199.94 & 212.11 & 218.84 & 221.96 & 231.45 & 233.80 \\
\hline 235.94 & 248.24 & 265.04 & 276.64 & 297.66 & 306.10 \\
\hline 317.65 & 337.14 & 340.99 & 349.65 & $366.20 \quad 4$ & 414.77 \\
\hline 473.67 & 489.62 & 513.75 & 514.39 & $527.53 \quad 5$ & 528.67 \\
\hline 533.63 & 535.06 & 543.83 & 554.83 & 558.52 & 572.24 \\
\hline 583.84 & 595.62 & 606.32 & 617.79 & 628.62 & 648.36 \\
\hline 682.59 & 706.52 & 716.69 & 721.43 & 749.33 & 763.90 \\
\hline 766.93 & 836.92 & 847.45 & 850.36 & 856.56 & 863.09 \\
\hline 872.04 & 880.12 & 891.67 & 903.41 & 913.74 & 922.19 \\
\hline 932.55 & 934.08 & 942.53 & 953.10 & 962.529 & 966.37 \\
\hline 994.85 & 999.37 & 1007.45 & 1016.14 & 41017.76 & 1025.80 \\
\hline 1031.33 & 1049.46 & $5 \quad 1049.90$ & $\begin{array}{ll}0 & 1057.90\end{array}$ & $0 \begin{array}{ll}0 & 1061.19\end{array}$ & $9 \quad 1065.95$ \\
\hline 1066.21 & 1067.04 & 41068.05 & $\begin{array}{ll}5 & 1083.98\end{array}$ & 81116.43 & $\begin{array}{ll}3 & 1122.22\end{array}$ \\
\hline 1159.95 & 1170.42 & 1198.30 & $\begin{array}{ll}0 & 1198.71\end{array}$ & 11206.51 & 11206.96 \\
\hline 1211.90 & 1224.96 & 1232.34 & $4 \quad 1247.10$ & $\begin{array}{ll}0 & 1265.67\end{array}$ & $\begin{array}{ll}7 & 1307.19\end{array}$ \\
\hline 1314.45 & 1321.03 & 31325.59 & 91334.51 & 11341.98 & $\begin{array}{ll}8 & 1345.57\end{array}$ \\
\hline 1363.90 & 1366.53 & 1369.23 & $\begin{array}{ll}3 & 1372.27\end{array}$ & $27 \quad 1385.25$ & $\begin{array}{ll}5 & 1395.24\end{array}$ \\
\hline 1401.26 & 1429.49 & 1430.23 & $\begin{array}{ll}3 & 1434.45\end{array}$ & $.5 \quad 1434.87$ & $\begin{array}{ll}7 & 1456.89\end{array}$ \\
\hline 1468.39 & 1468.59 & 1478.45 & 51482.85 & 31496.55 & $\begin{array}{ll}5 & 1496.97\end{array}$ \\
\hline 1499.06 & 1499.56 & 51505.70 & $\begin{array}{ll}0 & 1506.46\end{array}$ & 61513.05 & 51514.05 \\
\hline 1516.27 & 1525.31 & 1526.43 & $\begin{array}{ll}3 & 1531.10\end{array}$ & $\begin{array}{ll}0 & 1545.14\end{array}$ & 41622.94 \\
\hline 1651.20 & 1656.26 & 1658.31 & $1 \quad 1661.47$ & $7 \quad 1684.12$ & 23046.79 \\
\hline 3047.12 & 3047.72 & 2 3048.14 & 43076.97 & 73083.74 & 43108.09 \\
\hline 3108.40 & 3109.00 & 3110.90 & 03116.33 & 33128.65 & 53131.05 \\
\hline 3135.44 & 3135.81 & 3140.67 & 73147.08 & 83161.63 & $3 \quad 3171.44$ \\
\hline 3174.51 & 3175.62 & 23176.37 & $\begin{array}{ll}7 & 3181.37\end{array}$ & 733196.92 & 23207.63 \\
\hline 3252.02 & 3252.90 & 3474.15 & & & \\
\hline
\end{tabular}

${ }^{1} \mathbf{7 f}$

$\begin{array}{llllll}15.04 & 19.89 & 29.04 & 29.58 & 33.63 & 35.25\end{array}$

$\begin{array}{llllll}37.38 & 40.43 & 62.94 & 64.92 & 72.05 & 80.68\end{array}$

$\begin{array}{llllll}82.13 & 97.99 & 119.87 & 122.34 & 133.06 & 134.76\end{array}$

$\begin{array}{llllll}138.05 & 162.59 & 163.84 & 173.44 & 197.94 & 199.34\end{array}$

$\begin{array}{llllll}206.20 & 223.04 & 224.42 & 230.53 & 240.22 & 249.72\end{array}$

$\begin{array}{lllllll}258.77 & 274.90 & 279.46 & 287.12 & 290.41 & 298.56\end{array}$

$\begin{array}{llllll}310.54 & 312.45 & 324.65 & 334.88 & 341.08 & 342.04\end{array}$

$\begin{array}{lllllll}342.43 & 349.60 & 351.74 & 355.12 & 356.39 & 358.33\end{array}$

$\begin{array}{lllllll}361.04 & 405.56 & 408.11 & 409.00 & 411.96 & 417.51\end{array}$

$\begin{array}{llllll}436.05 & 446.43 & 450.69 & 452.62 & 463.82 & 464.07\end{array}$

$\begin{array}{llllll}493.58 & 498.57 & 528.78 & 529.36 & 536.00 & 545.04\end{array}$ $\begin{array}{llllll}549.00 & 562.57 & 565.78 & 583.51 & 583.65 & 610.08\end{array}$ $\begin{array}{lllllll}620.49 & 621.51 & 666.40 & 670.76 & 683.16 & 688.91\end{array}$ $\begin{array}{llllll}692.73 & 693.22 & 730.15 & 730.50 & 739.63 & 747.97\end{array}$ $\begin{array}{llllll}748.72 & 749.58 & 764.71 & 769.68 & 770.52 & 783.51\end{array}$ $\begin{array}{llllll}795.06 & 796.53 & 815.28 & 835.55 & 837.32 & 861.13\end{array}$ $\begin{array}{lllllll}873.64 & 876.41 & 885.11 & 889.23 & 896.30 & 896.78\end{array}$ $\begin{array}{lllllll}897.66 & 903.90 & 904.27 & 913.75 & 921.10 & 941.69\end{array}$ $\begin{array}{llllll}942.81 & 946.01 & 946.54 & 953.51 & 954.96 & 971.57\end{array}$ $\begin{array}{lllllll}972.43 & 979.49 & 979.78 & 999.49 & 1001.02 & 1012.68\end{array}$ $\begin{array}{lllllll}1012.72 & 1030.56 & 1036.53 & 1037.03 & 1043.29 & 1049.60\end{array}$ $\begin{array}{lllllll}1053.64 & 1056.84 & 1057.69 & 1059.67 & 1059.97 & 1071.43\end{array}$ $\begin{array}{lllllll}1077.20 & 1092.92 & 1094.61 & 1110.22 & 1143.64 & 1148.49\end{array}$ $\begin{array}{lllllll}1153.10 & 1160.12 & 1160.91 & 1162.87 & 1169.05 & 1190.98\end{array}$ $\begin{array}{llllll}1201.49 & 1202.10 & 1232.66 & 1232.93 & 1240.37 & 1243.35\end{array}$ $\begin{array}{llllll}1243.46 & 1244.25 & 1277.26 & 1292.81 & 1299.51 & 1302.03\end{array}$ $\begin{array}{lllllll}1309.21 & 1309.28 & 1310.13 & 1319.28 & 1319.78 & 1321.34\end{array}$ $\begin{array}{lllllll}1335.80 & 1342.75 & 1343.12 & 1344.09 & 1345.45 & 1354.38\end{array}$ $\begin{array}{llllllll}1355.00 & 1420.47 & 1422.25 & 1422.57 & 1422.80 & 1433.63\end{array}$ $\begin{array}{llllll}1436.14 & 1448.72 & 1456.40 & 1457.16 & 1459.63 & 1478.36\end{array}$ $\begin{array}{lllllll}1478.82 & 1498.29 & 1498.76 & 1499.76 & 1499.91 & 1503.29\end{array}$ $\begin{array}{lllllll}1504.98 & 1505.50 & 1508.22 & 1518.96 & 1521.03 & 1523.68\end{array}$ $\begin{array}{llllll}1525.16 & 1527.54 & 1527.68 & 1530.00 & 1531.00 & 1539.12\end{array}$ $\begin{array}{lllllll}1539.58 & 1591.06 & 1597.95 & 1605.63 & 1606.58 & 1619.60\end{array}$ $\begin{array}{lllllll}1620.10 & 1642.59 & 1646.10 & 1656.94 & 1664.17 & 1665.61\end{array}$ $\begin{array}{lllllll}1667.34 & 3042.74 & 3043.27 & 3043.87 & 3044.10 & 3049.33\end{array}$ $\begin{array}{lllllll}3049.82 & 3116.03 & 3116.44 & 3118.20 & 3118.93 & 3120.93\end{array}$ $\begin{array}{lllllll}3121.86 & 3122.21 & 3122.36 & 3123.08 & 3123.62 & 3128.91\end{array}$ $\begin{array}{lllllll}3129.45 & 3192.53 & 3193.04 & 3204.72 & 3205.35 & 3210.40\end{array}$ $\begin{array}{lllllll}3214.10 & 3215.55 & 3215.66 & 3220.63 & 3222.73 & 3223.64\end{array}$ $\begin{array}{lllllll}3231.02 & 3231.27 & 3232.52 & 3233.38 & 3236.83 & 3237.57\end{array}$ $3240.34 \quad 3243.24 \quad 3243.32$

\section{$==$
${ }^{3} \mathbf{7 f}$}

$\begin{array}{llllll}14.89 & 18.09 & 28.02 & 29.11 & 32.09 & 32.74\end{array}$ $\begin{array}{lllllll}38.53 & 41.90 & 63.47 & 65.17 & 68.68 & 74.73\end{array}$ $\begin{array}{lllllll}78.25 & 93.64 & 111.56 & 117.40 & 131.49 & 137.01\end{array}$ $\begin{array}{lllllll}140.60 & 157.32 & 165.06 & 172.97 & 178.92 & 192.54\end{array}$ $\begin{array}{llllll}196.05 & 207.25 & 222.52 & 232.44 & 240.32 & 252.76\end{array}$ $\begin{array}{lllllll}257.46 & 263.70 & 266.84 & 291.47 & 292.01 & 301.81\end{array}$ $\begin{array}{lllllll}304.55 & 315.31 & 323.80 & 333.23 & 338.49 & 339.43\end{array}$ $\begin{array}{lllllll}340.05 & 342.68 & 346.54 & 356.42 & 358.06 & 359.57\end{array}$ $\begin{array}{lllllll}360.13 & 396.63 & 402.12 & 404.52 & 405.94 & 415.70\end{array}$ $\begin{array}{lllllll}425.42 & 438.89 & 444.38 & 448.19 & 452.65 & 463.49\end{array}$ $\begin{array}{lllllll}468.03 & 490.25 & 527.03 & 528.54 & 532.17 & 536.81\end{array}$ $\begin{array}{lllllll}544.34 & 555.37 & 563.47 & 569.41 & 580.09 & 580.18\end{array}$ $\begin{array}{lllllll}585.08 & 588.66 & 654.10 & 666.54 & 671.45 & 672.96\end{array}$ $\begin{array}{llllll}689.70 & 693.18 & 710.67 & 717.84 & 726.94 & 730.11\end{array}$ $\begin{array}{llllll}737.36 & 741.34 & 745.65 & 746.99 & 770.30 & 772.07\end{array}$ $\begin{array}{lllllll}784.84 & 794.43 & 800.24 & 802.29 & 810.69 & 849.61\end{array}$ $\begin{array}{lllllll}850.05 & 858.19 & 874.22 & 887.88 & 892.60 & 893.81\end{array}$ $\begin{array}{lllllll}896.05 & 903.95 & 905.38 & 906.75 & 907.32 & 939.25\end{array}$ $\begin{array}{lllllll}939.77 & 950.69 & 951.53 & 952.84 & 954.65 & 961.35\end{array}$ $\begin{array}{llllll}961.52 & 977.17 & 977.41 & 986.86 & 991.59 & 1003.71\end{array}$ $\begin{array}{lllllll}1019.55 & 1020.56 & 1031.37 & 1032.29 & 1037.16 & 1042.75\end{array}$ $\begin{array}{lllllll}1052.14 & 1054.40 & 1055.52 & 1057.45 & 1061.04 & 1061.26\end{array}$ $\begin{array}{lllllll}1067.48 & 1069.39 & 1092.16 & 1095.60 & 1138.37 & 1139.18\end{array}$ $\begin{array}{lllllll}1141.70 & 1145.46 & 1159.93 & 1160.99 & 1164.21 & 1195.14\end{array}$ $\begin{array}{lllllll}1202.80 & 1202.89 & 1233.40 & 1233.71 & 1242.30 & 1242.98\end{array}$ $\begin{array}{lllllll}1243.88 & 1245.90 & 1263.12 & 1280.56 & 1283.23 & 1285.31\end{array}$ $\begin{array}{lllllll}1299.26 & 1308.73 & 1309.55 & 1316.54 & 1317.68 & 1326.80\end{array}$ $\begin{array}{lllllll}1329.66 & 1339.74 & 1341.70 & 1342.80 & 1356.07 & 1357.47\end{array}$ $\begin{array}{lllllll}1371.11 & 1415.40 & 1420.07 & 1420.15 & 1420.27 & 1421.25\end{array}$ $\begin{array}{lllllll}1425.76 & 1434.24 & 1452.46 & 1453.43 & 1473.17 & 1474.16\end{array}$ $\begin{array}{lllllll}1475.65 & 1495.39 & 1498.13 & 1498.38 & 1499.11 & 1499.71\end{array}$ $\begin{array}{lllllll}1500.27 & 1502.88 & 1505.27 & 1512.68 & 1517.75 & 1518.66\end{array}$ $\begin{array}{lllllll}1520.74 & 1520.86 & 1526.20 & 1526.88 & 1527.22 & 1530.16\end{array}$ $\begin{array}{lllllll}1538.87 & 1539.42 & 1564.64 & 1582.60 & 1590.03 & 1602.71\end{array}$ $\begin{array}{lllllll}1609.19 & 1612.52 & 1622.48 & 1626.56 & 1627.51 & 1659.05\end{array}$ $\begin{array}{lllllll}1659.25 & 3036.84 & 3039.05 & 3039.51 & 3040.39 & 3045.81\end{array}$ $\begin{array}{lllllll}3046.40 & 3109.60 & 3111.53 & 3113.47 & 3113.55 & 3114.22\end{array}$ $\begin{array}{llllll}3114.69 & 3116.27 & 3118.02 & 3120.81 & 3121.72 & 3122.96\end{array}$ $\begin{array}{lllllll}3123.31 & 3202.99 & 3203.72 & 3206.69 & 3209.22 & 3209.45\end{array}$ $\begin{array}{llllll}3209.52 & 3211.64 & 3217.06 & 3222.70 & 3224.99 & 3226.81\end{array}$ $\begin{array}{llllll}3228.92 & 3229.80 & 3231.05 & 3234.17 & 3236.13 & 3242.30\end{array}$ 
$3247.84 \quad 3247.98 \quad 3251.54$

$\mathbf{1}_{4}$

$\begin{array}{llllll}30.45 & 72.96 & 86.84 & 108.22 & 138.17 & 214.14\end{array}$ $\begin{array}{llllll}225.82 & 277.24 & 288.34 & 355.52 & 412.73 & 501.22\end{array}$ $\begin{array}{llllll}515.33 & 589.56 & 632.69 & 695.34 & 725.27 & 736.24\end{array}$ $\begin{array}{lllllll}783.76 & 850.32 & 853.24 & 888.72 & 933.34 & 961.88\end{array}$ $\begin{array}{lllllll}975.69 & 1001.73 & 1014.13 & 1037.01 & 1049.62 & 1061.28\end{array}$ $\begin{array}{llllll}1115.91 & 1179.01 & 1197.78 & 1205.83 & 1218.73 & 1223.25\end{array}$ $\begin{array}{lllllll}1240.85 & 1328.33 & 1358.31 & 1372.20 & 1375.31 & 1480.74\end{array}$ $\begin{array}{lllllll}1492.61 & 1493.54 & 1508.88 & 1541.24 & 1632.35 & 1659.80\end{array}$ $\begin{array}{llllll}1702.13 & 1795.94 & 3056.39 & 3130.17 & 3165.13 & 3167.85\end{array}$ $\begin{array}{lllllll}3176.93 & 3182.96 & 3192.75 & 3201.49 & 3208.63 & 3212.81\end{array}$

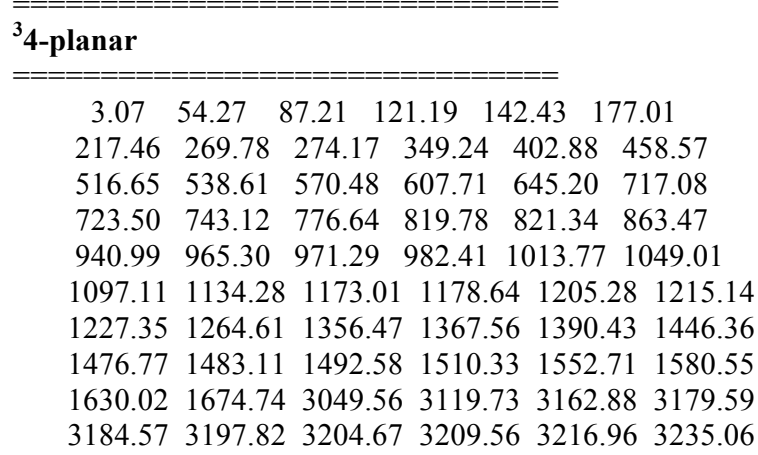

${ }^{3}$ 4-twisted

$\begin{array}{llllll}33.73 & 44.70 & 57.20 & 135.24 & 156.94 & 164.25\end{array}$

$\begin{array}{lllllll}248.33 & 256.93 & 317.70 & 351.56 & 409.26 & 485.23\end{array}$

$\begin{array}{lllllll}523.23 & 544.71 & 619.94 & 630.47 & 641.31 & 688.01\end{array}$

$\begin{array}{lllllll}719.21 & 744.71 & 769.58 & 834.74 & 835.19 & 900.68\end{array}$ $\begin{array}{lllllll}927.50 & 968.47 & 989.96 & 993.16 & 1029.83 & 1046.62\end{array}$ $\begin{array}{lllllll}1111.95 & 1131.50 & 1178.47 & 1189.80 & 1191.37 & 1194.95\end{array}$ $\begin{array}{lllllll}1215.33 & 1235.16 & 1333.61 & 1362.89 & 1382.19 & 1429.27\end{array}$ $\begin{array}{lllllll}1480.80 & 1492.49 & 1493.00 & 1508.93 & 1513.57 & 1595.38\end{array}$ $\begin{array}{lllllll}1618.05 & 1723.17 & 3052.97 & 3125.67 & 3131.25 & 3151.04\end{array}$ $\begin{array}{llllll}3165.48 & 3174.12 & 3178.78 & 3189.80 & 3195.93 & 3207.48\end{array}$

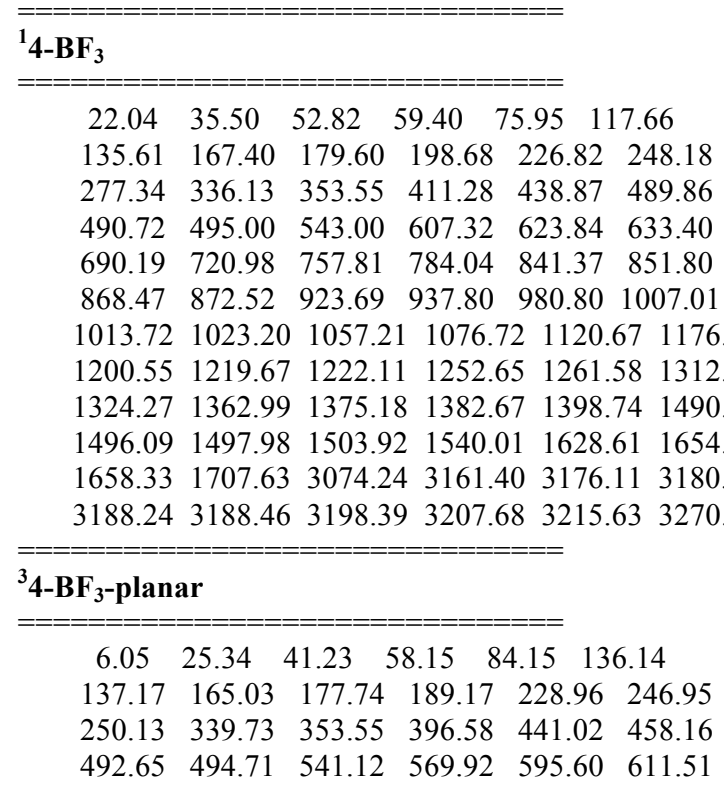

$\begin{array}{llllll}615.96 & 641.05 & 713.36 & 745.00 & 748.63 & 779.95\end{array}$ $\begin{array}{llllll}817.86 & 827.45 & 840.41 & 877.31 & 908.55 & 973.63\end{array}$ $\begin{array}{lllllll}975.23 & 989.88 & 1014.24 & 1081.18 & 1105.80 & 1143.97\end{array}$ $\begin{array}{llllll}1175.55 & 1176.33 & 1218.56 & 1227.04 & 1242.36 & 1271.03\end{array}$ $\begin{array}{lllllll}1308.32 & 1354.81 & 1367.52 & 1372.15 & 1408.51 & 1442.56\end{array}$ $\begin{array}{lllllll}1477.48 & 1493.52 & 1494.59 & 1503.93 & 1551.33 & 1574.13\end{array}$ $\begin{array}{lllllll}1595.27 & 1678.62 & 3063.19 & 3145.43 & 3179.62 & 3180.50\end{array}$ $\begin{array}{llllll}3186.76 & 3197.94 & 3207.85 & 3212.70 & 3224.02 & 3276.50\end{array}$

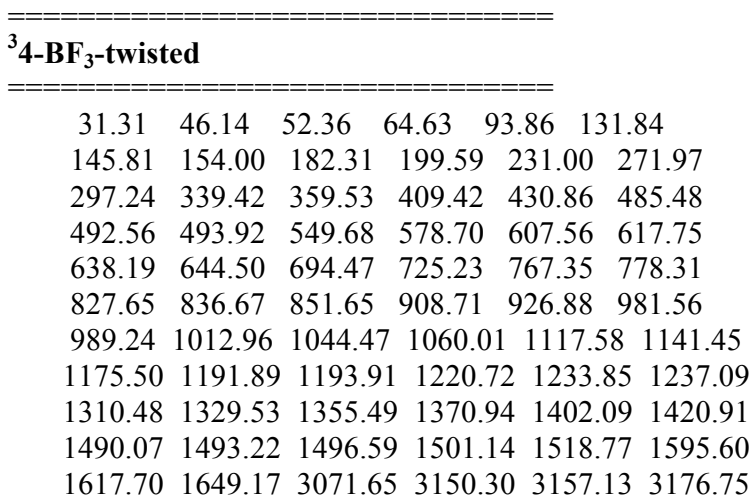
$\begin{array}{llllll}3183.76 & 3191.18 & 3191.78 & 3194.14 & 3198.85 & 3209.86\end{array}$

\begin{tabular}{|c|c|c|c|c|c|}
\hline${ }^{1}$ 4-AlEtCl ${ }_{2}$ & & & & & \\
\hline 18.12 & 20.15 & 31.23 & 38.04 & $39.73 \quad 72$ & 72.57 \\
\hline 77.13 & 103.91 & 110.88 & 124.22 & 134.08 & 157.02 \\
\hline 165.06 & 198.10 & 210.59 & 226.06 & 241.72 & 270.09 \\
\hline 278.85 & 280.63 & 367.19 & 406.15 & 410.75 & 450.82 \\
\hline 490.40 & 506.99 & 529.30 & 608.00 & 628.49 & 642.77 \\
\hline 650.37 & 688.85 & 725.16 & 749.50 & 785.85 & 851.97 \\
\hline 865.87 & 879.89 & 925.82 & 940.19 & 949.25 & 972.74 \\
\hline 983.85 & 1009.18 & 1009.90 & $\begin{array}{ll}0 & 1012.3\end{array}$ & 32 1023.9 & $97 \quad 1056.41$ \\
\hline 1080.57 & 1122.68 & $8 \quad 1175.3$ & 371201. & $.28 \quad 1219.9$ & $.97 \quad 1223.06$ \\
\hline 1245.56 & 1253.24 & 41264.9 & 1325 & $.60 \quad 1359.2$ & $.22 \quad 1378.49$ \\
\hline 1379.99 & 1401.47 & $\begin{array}{ll}7 & 1423.2\end{array}$ & 231465 & $\begin{array}{ll}88 & 1489.8\end{array}$ & $.87 \quad 1496.07$ \\
\hline 1498.55 & 1503.37 & $7 \quad 1515.0$ & 61522 & $.88 \quad 1540.1$ & .141625 .27 \\
\hline 1638.62 & 1657.82 & 21696.5 & 503017. & $.78 \quad 3022.2$ & $.23 \quad 3058.94$ \\
\hline 3073.93 & 3076.68 & $8 \quad 3093.9$ & 993160. & $.83 \quad 3175.6$ & .623180 .71 \\
\hline 3189.73 & 3190.37 & 73200.0 & 993208. & $\begin{array}{ll}83 & 3216.7\end{array}$ & $.73 \quad 3258.59$ \\
\hline
\end{tabular}

\section{${ }^{3}$ 4-AlEtCl -planar}

$\begin{array}{llllll}6.11 & 29.79 & 34.00 & 36.61 & 48.38 & 68.97\end{array}$ $\begin{array}{llllll}76.79 & 106.76 & 126.99 & 136.00 & 138.28 & 162.49\end{array}$ $\begin{array}{llllll}170.72 & 199.58 & 208.41 & 219.60 & 244.96 & 267.02\end{array}$ $\begin{array}{llllll}275.90 & 284.52 & 362.59 & 396.13 & 402.63 & 455.00\end{array}$ $\begin{array}{lllllll}459.61 & 498.68 & 528.18 & 583.64 & 599.45 & 601.62\end{array}$ $\begin{array}{llllll}638.16 & 643.66 & 650.02 & 735.91 & 742.15 & 755.90\end{array}$ $\begin{array}{llllll}783.91 & 815.66 & 819.01 & 882.07 & 904.43 & 950.16\end{array}$ $\begin{array}{lllllll}972.35 & 972.98 & 977.60 & 990.66 & 1012.25 & 1013.22\end{array}$ $\begin{array}{llllll}1080.45 & 1108.94 & 1149.54 & 1175.54 & 1178.20 & 1218.39\end{array}$ $\begin{array}{llllll}1228.13 & 1246.19 & 1265.26 & 1268.92 & 1352.26 & 1369.79\end{array}$ $\begin{array}{lllllll}1372.26 & 1407.63 & 1423.94 & 1440.31 & 1466.05 & 1475.19\end{array}$ $\begin{array}{llllll}1494.45 & 1496.14 & 1503.00 & 1515.70 & 1522.86 & 1549.39\end{array}$ $\begin{array}{lllllll}1564.17 & 1589.97 & 1679.62 & 3012.70 & 3017.15 & 3053.70\end{array}$ $\begin{array}{lllllll}3055.56 & 3071.87 & 3089.52 & 3138.60 & 3172.38 & 3184.48\end{array}$ $\begin{array}{llllll}3193.11 & 3201.83 & 3211.44 & 3214.08 & 3224.56 & 3240.06\end{array}$

\section{${ }^{3}$ 4-AlEtCl ${ }_{2}$-twisted}


$\begin{array}{llllll}17.68 & 24.74 & 36.61 & 41.84 & 52.26 & 57.17\end{array}$ $\begin{array}{llllll}76.08 & 99.61 & 114.84 & 137.23 & 150.47 & 156.62\end{array}$ $\begin{array}{llllll}161.69 & 177.22 & 212.10 & 235.17 & 247.33 & 263.95\end{array}$ $\begin{array}{llllll}273.06 & 309.30 & 368.57 & 401.59 & 405.99 & 439.55\end{array}$ $\begin{array}{llllll}483.99 & 510.20 & 546.48 & 561.45 & 606.30 & 625.22\end{array}$ $\begin{array}{lllllll}643.33 & 647.86 & 654.99 & 688.11 & 715.70 & 766.54\end{array}$ $\begin{array}{llllll}775.29 & 839.22 & 841.93 & 911.58 & 914.28 & 949.54\end{array}$ $\begin{array}{lllllll}972.72 & 974.81 & 993.64 & 999.04 & 1011.85 & 1044.66\end{array}$ $\begin{array}{lllllll}1059.36 & 1117.58 & 1139.13 & 1173.29 & 1192.21 & 1194.95\end{array}$ $\begin{array}{lllllll}1220.61 & 1233.16 & 1245.30 & 1264.81 & 1323.58 & 1349.54\end{array}$ $\begin{array}{lllllll}1367.99 & 1397.76 & 1422.59 & 1424.88 & 1464.98 & 1490.19\end{array}$ $\begin{array}{llllllll}1492.25 & 1495.97 & 1498.97 & 1515.75 & 1516.48 & 1522.60\end{array}$ $\begin{array}{lllllll}1595.13 & 1617.96 & 1635.80 & 3018.13 & 3022.75 & 3059.21\end{array}$ $\begin{array}{llllll}3072.10 & 3077.02 & 3093.72 & 3142.18 & 3158.36 & 3161.63\end{array}$ $\begin{array}{llllll}3176.99 & 3185.77 & 3193.36 & 3193.48 & 3200.58 & 3211.08\end{array}$

\section{${ }^{1} 4-\mathrm{AlBr}_{3}$}

$\begin{array}{llllll}14.03 & 18.25 & 28.06 & 32.78 & 61.92 & 71.73\end{array}$ $\begin{array}{llllll}86.59 & 101.42 & 106.75 & 112.48 & 137.22 & 145.79\end{array}$ $\begin{array}{llllll}191.92 & 198.43 & 223.34 & 225.13 & 278.88 & 300.03\end{array}$ $\begin{array}{lllllll}369.66 & 408.56 & 434.23 & 462.48 & 471.09 & 487.74\end{array}$ $\begin{array}{lllllll}530.19 & 608.86 & 630.63 & 687.90 & 725.79 & 753.45\end{array}$ $\begin{array}{llllll}786.03 & 850.57 & 871.85 & 874.96 & 930.52 & 939.86\end{array}$ $\begin{array}{lllllll}979.01 & 1010.14 & 1012.94 & 1024.57 & 1056.23 & 1078.80\end{array}$ $\begin{array}{lllllll}1123.31 & 1173.14 & 1202.44 & 1220.00 & 1223.73 & 1255.32\end{array}$ $\begin{array}{lllllll}1325.59 & 1356.26 & 1377.41 & 1380.00 & 1413.01 & 1489.93\end{array}$ $\begin{array}{lllllll}1496.29 & 1498.03 & 1505.25 & 1540.34 & 1612.17 & 1629.36\end{array}$ $\begin{array}{llllllll}1655.54 & 1687.42 & 3080.44 & 3169.79 & 3176.36 & 3181.68\end{array}$ $\begin{array}{llllll}3190.93 & 3199.36 & 3201.14 & 3209.44 & 3217.16 & 3253.71\end{array}$

\section{${ }^{3} 4-\mathrm{AlBr}_{3}$-planar}

$\begin{array}{llllll}4.96 & 20.46 & 29.06 & 35.20 & 60.99 & 64.94\end{array}$ $\begin{array}{llllll}86.94 & 101.83 & 107.54 & 134.67 & 140.09 & 163.17\end{array}$ $\begin{array}{llllll}186.05 & 194.52 & 211.45 & 222.12 & 278.29 & 300.39\end{array}$ $\begin{array}{lllllll}360.79 & 392.84 & 420.61 & 458.70 & 462.44 & 485.81\end{array}$ $\begin{array}{lllllll}529.53 & 584.55 & 592.37 & 601.21 & 637.78 & 744.66\end{array}$ $\begin{array}{llllll}746.44 & 752.78 & 784.13 & 818.29 & 829.97 & 883.82\end{array}$ $\begin{array}{lllllll}910.30 & 975.49 & 976.17 & 992.60 & 1013.00 & 1079.86\end{array}$ $\begin{array}{lllllll}1109.68 & 1150.14 & 1172.24 & 1179.89 & 1218.46 & 1227.87\end{array}$ $\begin{array}{lllllll}1269.87 & 1351.80 & 1365.26 & 1371.60 & 1414.33 & 1437.83\end{array}$ $\begin{array}{lllllll}1473.67 & 1494.65 & 1495.30 & 1502.81 & 1546.07 & 1557.64\end{array}$ $\begin{array}{lllllll}1586.90 & 1674.33 & 3064.75 & 3149.15 & 3184.06 & 3184.66\end{array}$ $\begin{array}{lllllll}3191.93 & 3201.51 & 3211.47 & 3213.70 & 3222.01 & 3246.64\end{array}$

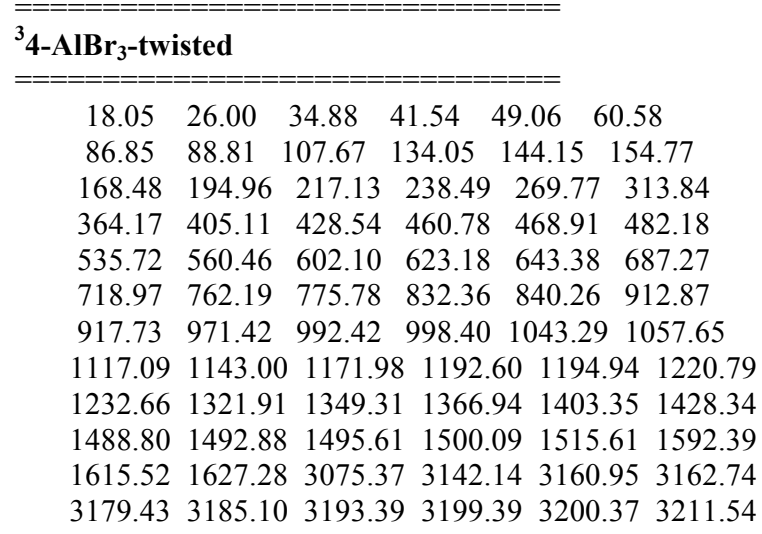

${ }^{1}$ 4-LA $\begin{array}{llllll}27.96 & 29.47 & 38.53 & 43.28 & 45.51 & 48.43\end{array}$ $\begin{array}{llllll}48.69 & 55.17 & 60.10 & 62.07 & 63.04 & 79.05\end{array}$ $\begin{array}{llllll}89.00 & 89.26 & 90.20 & 93.50 & 94.70 & 105.03\end{array}$ $\begin{array}{llllll}112.41 & 128.85 & 135.66 & 142.44 & 170.42 & 177.49\end{array}$ $\begin{array}{lllllll}180.80 & 194.74 & 199.13 & 207.47 & 211.12 & 220.42\end{array}$ $\begin{array}{llllll}225.90 & 228.13 & 232.28 & 235.49 & 243.32 & 248.58\end{array}$ $\begin{array}{lllllll}252.00 & 252.95 & 274.49 & 281.08 & 294.02 & 302.46\end{array}$ $\begin{array}{lllllll}304.37 & 316.09 & 333.15 & 344.50 & 356.49 & 371.05\end{array}$ $\begin{array}{lllllll}379.13 & 394.99 & 409.95 & 427.13 & 471.94 & 482.48\end{array}$ $\begin{array}{lllllll}489.41 & 505.75 & 507.05 & 517.81 & 528.65 & 532.49\end{array}$ $\begin{array}{lllllll}533.74 & 536.80 & 538.97 & 549.82 & 555.06 & 560.61\end{array}$ $\begin{array}{llllll}576.36 & 577.74 & 603.43 & 615.38 & 619.55 & 621.98\end{array}$ $\begin{array}{lllllll}630.15 & 636.88 & 663.94 & 674.45 & 689.34 & 711.24\end{array}$ $\begin{array}{llllll}717.28 & 723.37 & 725.30 & 754.34 & 765.21 & 772.46\end{array}$ $\begin{array}{lllllll}783.93 & 796.44 & 845.03 & 846.30 & 851.04 & 858.46\end{array}$ $\begin{array}{lllllll}861.37 & 865.73 & 872.75 & 875.97 & 884.73 & 890.02\end{array}$ $\begin{array}{llllll}902.06 & 908.29 & 916.72 & 924.28 & 927.46 & 934.42\end{array}$ $\begin{array}{llllll}940.50 & 946.39 & 950.61 & 951.72 & 963.55 & 966.59\end{array}$ $\begin{array}{lllllll}987.33 & 992.08 & 1001.90 & 1013.00 & 1013.88 & 1014.91\end{array}$ $\begin{array}{lllllll}1018.93 & 1019.77 & 1033.17 & 1035.19 & 1035.65 & 1044.89\end{array}$ $\begin{array}{llllll}1048.29 & 1049.88 & 1055.35 & 1055.55 & 1064.23 & 1065.29\end{array}$ $\begin{array}{lllllll}1067.58 & 1070.86 & 1079.75 & 1090.28 & 1110.95 & 1119.00\end{array}$ $\begin{array}{lllllll}1124.83 & 1128.17 & 1156.40 & 1166.70 & 1184.67 & 1191.73\end{array}$ $\begin{array}{lllllll}1198.07 & 1203.24 & 1205.05 & 1207.74 & 1209.78 & 1221.36\end{array}$ $\begin{array}{lllllll}1223.10 & 1226.33 & 1233.63 & 1258.79 & 1261.90 & 1266.69\end{array}$ $\begin{array}{llllll}1302.16 & 1317.06 & 1323.98 & 1327.03 & 1330.46 & 1337.41\end{array}$ $\begin{array}{lllllll}1340.49 & 1346.10 & 1355.66 & 1360.08 & 1366.51 & 1371.91\end{array}$ $\begin{array}{lllllll}1372.58 & 1374.00 & 1377.82 & 1382.41 & 1386.15 & 1405.07\end{array}$ $\begin{array}{llllll}1424.65 & 1427.75 & 1431.34 & 1433.54 & 1437.35 & 1462.83\end{array}$ $\begin{array}{lllllll}1467.18 & 1470.59 & 1477.00 & 1482.86 & 1489.78 & 1497.72\end{array}$ $\begin{array}{lllllll}1498.63 & 1499.45 & 1501.53 & 1505.88 & 1507.30 & 1507.79\end{array}$ $\begin{array}{llllll}1512.80 & 1513.58 & 1514.68 & 1516.64 & 1520.33 & 1525.30\end{array}$ $\begin{array}{lllllll}1526.36 & 1534.76 & 1541.53 & 1585.78 & 1626.14 & 1631.24\end{array}$ $\begin{array}{lllllll}1652.18 & 1653.60 & 1656.39 & 1657.64 & 1663.24 & 1676.25\end{array}$ $\begin{array}{lllllll}1684.65 & 3040.11 & 3044.47 & 3051.21 & 3056.90 & 3068.58\end{array}$ $\begin{array}{lllllll}3080.78 & 3101.99 & 3103.64 & 3105.95 & 3107.51 & 3107.69\end{array}$ $\begin{array}{lllllll}3111.75 & 3120.17 & 3132.06 & 3132.16 & 3132.44 & 3132.53\end{array}$ $\begin{array}{llllll}3134.93 & 3165.95 & 3169.32 & 3171.22 & 3171.92 & 3173.56\end{array}$ $\begin{array}{lllllll}3174.80 & 3178.10 & 3185.31 & 3189.19 & 3196.90 & 3197.62\end{array}$ $\begin{array}{lllllll}3201.35 & 3207.65 & 3217.82 & 3222.77 & 3226.57 & 3247.89\end{array}$ $3250.67 \quad 3281.12 \quad 3510.26$

\begin{tabular}{|c|c|c|c|c|c|}
\hline \multicolumn{5}{|l|}{${ }^{3} 4-\mathrm{LA}$} & \multirow[b]{2}{*}{32.12} \\
\hline 13.40 & 19.20 & 22.82 & 24.23 & 30.98 & \\
\hline 36.19 & 39.67 & 44.18 & 47.29 & 51.51 & 56.77 \\
\hline 60.84 & 61.67 & 65.04 & 79.00 & 88.14 & 109.15 \\
\hline 112.44 & 121.16 & 133.67 & 145.27 & $7 \quad 160.72$ & $2 \quad 173.20$ \\
\hline 180.94 & 188.41 & 189.57 & 200.29 & 207.44 & 210.82 \\
\hline 216.01 & 221.91 & 226.46 & 230.21 & 235.07 & 242.79 \\
\hline 246.88 & 250.51 & 264.70 & 276.27 & 281.40 & 298.43 \\
\hline 307.02 & 322.95 & 328.76 & 347.85 & 352.80 & 363.94 \\
\hline 372.77 & 387.77 & 399.38 & 423.13 & 466.25 & 470.71 \\
\hline 481.21 & 492.37 & 503.98 & 509.56 & 522.72 & 528.25 \\
\hline 531.33 & 532.34 & 538.13 & 540.12 & 2551.91 & 556.03 \\
\hline 569.56 & 570.41 & 594.72 & 600.42 & 608.66 & 611.09 \\
\hline 615.17 & 620.03 & 632.53 & 655.09 & 662.88 & 674.53 \\
\hline 701.95 & 712.22 & 717.06 & 746.22 & 2747.94 & 756.74 \\
\hline 767.09 & 784.11 & 802.34 & 813.63 & 821.44 & 840.51 \\
\hline 841.62 & 845.19 & 855.02 & 862.88 & 868.25 & 875.37 \\
\hline 878.87 & 897.81 & 903.49 & 908.12 & 2912.33 & 3916.97 \\
\hline
\end{tabular}


$\begin{array}{llllll}927.95 & 931.80 & 936.40 & 945.34 & 947.37 & 960.63\end{array}$ $\begin{array}{llllll}965.27 & 981.03 & 982.50 & 987.61 & 998.02 & 998.96\end{array}$ $\begin{array}{lllllll}1011.01 & 1013.42 & 1016.03 & 1025.45 & 1025.70 & 1034.97\end{array}$ $\begin{array}{llllll}1040.59 & 1045.24 & 1046.01 & 1050.60 & 1057.46 & 1058.42\end{array}$ $\begin{array}{lllllll}1064.86 & 1065.25 & 1065.79 & 1091.12 & 1104.17 & 1113.00\end{array}$ $\begin{array}{lllllll}1116.70 & 1123.76 & 1154.54 & 1157.48 & 1169.73 & 1175.04\end{array}$ $\begin{array}{lllllll}1188.83 & 1190.86 & 1193.72 & 1198.30 & 1201.83 & 1205.20\end{array}$ $\begin{array}{lllllll}1217.30 & 1218.77 & 1220.83 & 1224.35 & 1261.31 & 1263.19\end{array}$ $\begin{array}{lllllll}1268.26 & 1294.99 & 1314.75 & 1322.78 & 1330.37 & 1333.88\end{array}$ $\begin{array}{lllllll}1337.32 & 1339.02 & 1348.98 & 1352.95 & 1357.70 & 1368.11\end{array}$ $\begin{array}{lllllll}1368.77 & 1370.51 & 1373.66 & 1378.34 & 1385.77 & 1401.81\end{array}$ $\begin{array}{lllllll}1419.06 & 1424.24 & 1427.87 & 1428.41 & 1430.79 & 1443.91\end{array}$ $\begin{array}{lllllll}1455.29 & 1460.62 & 1467.23 & 1469.33 & 1476.91 & 1480.85\end{array}$ $\begin{array}{lllllll}1494.29 & 1495.52 & 1497.42 & 1498.39 & 1501.36 & 1502.30\end{array}$ $\begin{array}{lllllll}1504.62 & 1505.82 & 1511.23 & 1512.75 & 1515.12 & 1517.81\end{array}$ $\begin{array}{lllllll}1524.16 & 1524.73 & 1531.76 & 1535.48 & 1556.77 & 1588.33\end{array}$ $\begin{array}{lllllll}1623.24 & 1629.45 & 1641.29 & 1652.01 & 1659.41 & 1661.13\end{array}$ $\begin{array}{lllllll}1677.79 & 3037.96 & 3039.43 & 3043.24 & 3045.58 & 3066.44\end{array}$ $\begin{array}{lllllll}3069.54 & 3099.02 & 3100.94 & 3102.96 & 3106.41 & 3108.66\end{array}$ $\begin{array}{llllll}3112.32 & 3115.36 & 3127.98 & 3130.02 & 3131.06 & 3132.06\end{array}$ $\begin{array}{lllllll}3143.57 & 3155.81 & 3165.91 & 3171.00 & 3173.43 & 3176.25\end{array}$ $\begin{array}{lllllll}3176.56 & 3179.00 & 3189.59 & 3191.71 & 3195.18 & 3204.36\end{array}$ $\begin{array}{lllllll}3206.72 & 3212.61 & 3214.11 & 3219.75 & 3222.44 & 3248.82\end{array}$ $3249.08 \quad 3282.79 \quad 3513.62$

\footnotetext{
14 a-LA

$\begin{array}{llllll}12.96 & 20.89 & 24.87 & 26.68 & 30.61 & 33.55\end{array}$

$\begin{array}{llllll}38.43 & 42.32 & 44.29 & 46.86 & 50.63 & 59.32\end{array}$

$\begin{array}{llllll}63.24 & 65.94 & 73.09 & 73.94 & 94.10 & 99.20\end{array}$

$\begin{array}{llllll}116.91 & 131.57 & 136.35 & 154.29 & 169.40 & 178.86\end{array}$ $\begin{array}{lllllll}188.97 & 200.18 & 207.36 & 208.37 & 214.91 & 224.77\end{array}$ $\begin{array}{llllll}225.59 & 228.85 & 229.58 & 236.46 & 250.27 & 255.85\end{array}$ $\begin{array}{llllll}256.48 & 263.28 & 279.45 & 289.30 & 315.32 & 319.36\end{array}$ $\begin{array}{llllll}321.90 & 330.23 & 334.01 & 335.46 & 354.22 & 370.53\end{array}$ $\begin{array}{llllll}409.83 & 410.63 & 418.76 & 470.88 & 487.17 & 492.61\end{array}$ $\begin{array}{lllllll}500.53 & 509.49 & 517.83 & 528.11 & 529.74 & 531.11\end{array}$ $\begin{array}{lllllll}532.89 & 546.07 & 552.39 & 558.19 & 558.53 & 577.54\end{array}$ $\begin{array}{llllll}580.75 & 596.58 & 602.06 & 610.45 & 616.38 & 624.31\end{array}$ $\begin{array}{lllllll}628.73 & 630.26 & 656.43 & 668.24 & 689.19 & 705.74\end{array}$ $\begin{array}{llllll}712.47 & 721.40 & 724.66 & 753.01 & 765.55 & 770.04\end{array}$ $\begin{array}{llllll}783.59 & 787.09 & 842.65 & 845.18 & 853.80 & 855.85\end{array}$ $\begin{array}{lllllll}860.35 & 866.07 & 870.17 & 877.76 & 881.37 & 884.14\end{array}$ $\begin{array}{llllll}895.86 & 909.22 & 914.55 & 924.26 & 931.72 & 933.10\end{array}$ $\begin{array}{llllll}949.22 & 951.78 & 954.46 & 962.15 & 967.25 & 981.10\end{array}$ $\begin{array}{llllll}989.40 & 997.38 & 1006.07 & 1013.61 & 1015.49 & 1020.46\end{array}$ $\begin{array}{lllllll}1020.83 & 1021.75 & 1038.58 & 1039.86 & 1046.60 & 1047.45\end{array}$ $\begin{array}{lllllll}1053.21 & 1056.35 & 1058.45 & 1064.09 & 1065.64 & 1066.90\end{array}$ $\begin{array}{lllllll}1072.23 & 1085.11 & 1112.47 & 1117.47 & 1124.80 & 1131.17\end{array}$ $\begin{array}{lllllll}1139.76 & 1154.78 & 1159.17 & 1166.71 & 1184.20 & 1191.54\end{array}$ $\begin{array}{lllllll}1198.71 & 1205.01 & 1205.66 & 1206.11 & 1213.17 & 1222.14\end{array}$ $\begin{array}{lllllll}1223.85 & 1225.98 & 1253.30 & 1260.97 & 1264.28 & 1271.43\end{array}$ $\begin{array}{lllllll}1291.84 & 1305.47 & 1318.01 & 1322.98 & 1331.32 & 1335.79\end{array}$ $\begin{array}{lllllll}1339.22 & 1345.55 & 1350.08 & 1353.22 & 1366.60 & 1368.93\end{array}$ $\begin{array}{lllllll}1369.91 & 1377.53 & 1380.84 & 1392.26 & 1394.00 & 1404.22\end{array}$ $\begin{array}{lllllll}1426.83 & 1427.81 & 1428.03 & 1430.81 & 1466.09 & 1468.81\end{array}$ $\begin{array}{llllll}1470.25 & 1481.75 & 1485.80 & 1489.72 & 1492.00 & 1497.73\end{array}$ $\begin{array}{lllllll}1500.54 & 1501.91 & 1503.53 & 1504.86 & 1506.23 & 1509.84\end{array}$ $\begin{array}{lllllll}1511.23 & 1515.65 & 1516.73 & 1524.11 & 1525.58 & 1529.41\end{array}$ $\begin{array}{lllllll}1529.56 & 1542.96 & 1543.74 & 1588.19 & 1626.56 & 1633.23\end{array}$ $\begin{array}{lllllll}1650.87 & 1655.82 & 1656.71 & 1657.93 & 1661.92 & 1679.93\end{array}$ $\begin{array}{lllllll}1680.52 & 2616.93 & 2651.03 & 2723.21 & 3038.35 & 3043.02\end{array}$
}

$\begin{array}{llllll}3045.34 & 3070.07 & 3072.11 & 3085.34 & 3099.66 & 3103.22\end{array}$ $\begin{array}{lllllll}3107.51 & 3114.26 & 3124.33 & 3127.49 & 3128.64 & 3129.96\end{array}$ $\begin{array}{lllllll}3132.64 & 3157.16 & 3160.60 & 3170.10 & 3170.99 & 3174.79\end{array}$ $\begin{array}{lllllll}3176.36 & 3176.56 & 3192.27 & 3196.41 & 3197.47 & 3197.71\end{array}$ $\begin{array}{llllll}3203.96 & 3212.66 & 3216.11 & 3219.75 & 3226.42 & 3235.09\end{array}$ $3248.16 \quad 3249.30 \quad 3509.43$

\begin{tabular}{|c|c|c|c|c|}
\hline${ }^{3} 4 a-L A$ & & & & \\
\hline 10.32 & 15.28 & 24.34 & 26.56 & 6.58 \\
\hline 38.62 & 44.53 & 46.68 & $53.86 \quad 58.96$ & 0.16 \\
\hline 67.04 & 73.88 & 82.90 & $93.17 \quad 104.09$ & 17.88 \\
\hline 121.04 & 127.79 & 137.89 & $158.23 \quad 174.03$ & 178.24 \\
\hline 188.30 & 195.56 & 211.67 & 214.16 & 222.16 \\
\hline 224.72 & 228.01 & 235.77 & 243.21 & 257.41 \\
\hline 260.71 & 271.33 & 278.53 & 288.88 & 307.41 \\
\hline 321.99 & 328.44 & 332.01 & 360.19 & 368.41 \\
\hline 385.80 & 398.78 & 425.10 & 479.58 & 499.02 \\
\hline 504.45 & 510.96 & 520.78 & 528.43 & 529.99 \\
\hline 535.42 & 539.55 & 551.87 & $\begin{array}{lll}556.27 & 559.68 \quad 5\end{array}$ & 572.23 \\
\hline 576.43 & 584.63 & 598.24 & $604.08 \quad 605.80 \quad 6$ & 614.04 \\
\hline 629.73 & 632.03 & 649.63 & $656.51 \quad 669.20$ & 706.93 \\
\hline 711.79 & 717.82 & 725.13 & 755.77 & 767.65 \\
\hline 774.38 & 792.90 & 803.55 & $811.37 \quad 838.96$ & 841.23 \\
\hline 846.92 & 850.67 & 855.85 & $\begin{array}{lll}861.65 & 870.77 & 8\end{array}$ & 879.13 \\
\hline 883.51 & 893.37 & 899.39 & $905.86912 .94 \quad 9$ & 925.37 \\
\hline 929.76 & 931.27 & 945.54 & $954.39 \quad 962.12$ & 966.85 \\
\hline 973.44 & 978.94 & 983.23 & $\begin{array}{lll}996.25 & 1003.68 & 1\end{array}$ & 1005.92 \\
\hline 1012.48 & 1014.79 & $9 \quad 1021.31$ & $\begin{array}{lll}31 & 1023.16 & 1030.23\end{array}$ & $23 \quad 1037.54$ \\
\hline 1045.51 & 1046.28 & $8 \quad 1052.13$ & $13 \quad 1057.29 \quad 1059.38$ & $38 \quad 1065.59$ \\
\hline 1066.38 & 1073.73 & $\begin{array}{ll}3 & 1107.93\end{array}$ & $33 \quad 1114.19 \quad 1117.24$ & $24 \quad 1125.88$ \\
\hline 1151.53 & 1156.33 & 31170.95 & $95 \quad 1174.11 \quad 1188.97$ & 971192.62 \\
\hline 1193.57 & 1196.98 & $8 \quad 1204.96$ & $96 \quad 1205.97 \quad 1216.13$ & 131217.92 \\
\hline 1220.17 & 1222.84 & $4 \quad 1229.72$ & $\begin{array}{lll}72 & 1236.48 & 1260.28\end{array}$ & $28 \quad 1263.03$ \\
\hline 1273.07 & 1297.83 & 31303.67 & $\begin{array}{llll}67 & 1315.37 & 1323.02\end{array}$ & $02 \quad 1331.96$ \\
\hline 1336.24 & 1341.07 & $7 \quad 1345.04$ & $94 \quad 1355.78 \quad 1361.20$ & 201368.74 \\
\hline 1373.26 & 1374.76 & $\begin{array}{ll}6 & 1374.98\end{array}$ & $88 \quad 1377.12 \quad 1386.64$ & $64 \quad 1403.38$ \\
\hline 1411.78 & 1426.51 & 11427.87 & $\begin{array}{lll}37 & 1430.92 & 1453.24\end{array}$ & 241456.65 \\
\hline 1462.82 & 1468.22 & 21480.15 & $\begin{array}{lll}15 & 1481.41 & 1482.45\end{array}$ & $45 \quad 1496.43$ \\
\hline 1497.13 & 1498.01 & 11500.85 & $\begin{array}{llll}35 & 1502.83 & 1504.92\end{array}$ & $92 \quad 1507.12$ \\
\hline 1513.15 & 1513.99 & $9 \quad 1515.73$ & $3 \quad 1517.95 \quad 1518.40$ & $40 \quad 1524.92$ \\
\hline 1526.16 & 1533.67 & 71542.86 & $\begin{array}{llll}36 & 1547.15 & 1554.35\end{array}$ & 351584.81 \\
\hline 1623.36 & 1630.46 & 61641.08 & $8 \quad 1650.77 \quad 1654.95$ & 951658.39 \\
\hline 1678.15 & 1886.17 & 72003.33 & $\begin{array}{lll}3 & 2099.31 & 3038.57\end{array}$ & $57 \quad 3042.79$ \\
\hline 3042.87 & 3063.60 & $\begin{array}{ll}0 & 3066.71\end{array}$ & $\begin{array}{lll}1 & 3099.40 & 3101.12\end{array}$ & 123102.62 \\
\hline 3106.98 & 3108.29 & 93116.67 & $\begin{array}{lll}7 & 3127.41 & 3130.22\end{array}$ & 223131.64 \\
\hline 3135.28 & 3146.77 & 73164.53 & $\begin{array}{lll}3 & 3168.75 & 3170.48\end{array}$ & $48 \quad 3173.57$ \\
\hline 3176.04 & 3177.95 & 53185.87 & $373188.11 \quad 3197.17$ & 173206.78 \\
\hline 3213.05 & 3216.28 & $8 \quad 3222.16$ & $\begin{array}{ll}6 & 3222.77 \quad 3229.16\end{array}$ & 163248.10 \\
\hline 3248.45 & 3280.09 & 93514.93 & & \\
\hline
\end{tabular}

14b-LA

$\begin{array}{llllll}14.97 & 22.20 & 28.16 & 32.22 & 33.34 & 38.18\end{array}$ $\begin{array}{lllllll}41.49 & 44.86 & 49.57 & 52.28 & 60.71 & 64.36\end{array}$ $\begin{array}{llllll}72.31 & 75.25 & 77.80 & 89.36 & 92.81 & 99.71\end{array}$ $\begin{array}{llllll}111.39 & 122.06 & 131.61 & 135.69 & 144.93 & 172.34\end{array}$ $\begin{array}{llllll}182.99 & 185.50 & 191.64 & 194.96 & 208.59 & 212.68\end{array}$ $\begin{array}{lllllll}215.08 & 223.60 & 227.99 & 230.03 & 235.52 & 238.06\end{array}$ $\begin{array}{llllll}246.17 & 247.93 & 260.88 & 279.57 & 284.73 & 292.86\end{array}$ $\begin{array}{lllllll}307.44 & 310.57 & 327.70 & 331.31 & 341.54 & 357.31\end{array}$ $\begin{array}{llllll}380.98 & 400.85 & 409.19 & 420.54 & 459.39 & 478.19\end{array}$ 
$\begin{array}{llllll}486.91 & 500.93 & 518.42 & 519.38 & 526.54 & 531.64\end{array}$ $\begin{array}{lllllll}532.46 & 534.74 & 542.52 & 548.85 & 553.47 & 568.84\end{array}$ $\begin{array}{lllllll}580.40 & 588.11 & 605.31 & 616.65 & 623.77 & 627.83\end{array}$ $\begin{array}{llllll}629.28 & 638.24 & 668.70 & 678.13 & 688.92 & 706.64\end{array}$ $\begin{array}{llllll}712.36 & 718.83 & 724.10 & 734.72 & 755.63 & 768.09\end{array}$ $\begin{array}{lllllll}783.07 & 789.34 & 830.82 & 843.06 & 848.37 & 850.33\end{array}$ $\begin{array}{llllll}856.08 & 864.13 & 867.97 & 870.83 & 873.54 & 877.81\end{array}$ $\begin{array}{llllll}891.96 & 899.22 & 908.75 & 911.88 & 912.64 & 923.69\end{array}$ $\begin{array}{lllllll}940.55 & 942.89 & 957.00 & 960.01 & 965.52 & 976.26\end{array}$ $\begin{array}{lllllll}986.80 & 1008.65 & 1011.01 & 1011.78 & 1014.53 & 1015.42\end{array}$ $\begin{array}{llllll}1018.19 & 1022.65 & 1024.31 & 1037.90 & 1046.15 & 1046.87\end{array}$ $\begin{array}{lllllll}1049.38 & 1054.36 & 1059.02 & 1062.41 & 1062.62 & 1065.04\end{array}$ $\begin{array}{lllllll}1065.81 & 1068.00 & 1082.00 & 1096.62 & 1105.38 & 1121.74\end{array}$ $\begin{array}{llllll}1122.44 & 1130.16 & 1156.66 & 1173.07 & 1181.05 & 1192.20\end{array}$ $\begin{array}{lllllll}1197.81 & 1204.00 & 1204.43 & 1210.51 & 1216.17 & 1219.57\end{array}$ $\begin{array}{lllllll}1223.40 & 1235.48 & 1255.14 & 1257.15 & 1263.66 & 1275.51\end{array}$ $\begin{array}{llllll}1304.26 & 1310.65 & 1312.85 & 1315.88 & 1329.58 & 1335.03\end{array}$ $\begin{array}{lllllll}1338.42 & 1340.80 & 1342.84 & 1348.35 & 1364.62 & 1368.75\end{array}$ $\begin{array}{lllllll}1371.39 & 1377.32 & 1382.19 & 1383.67 & 1398.16 & 1414.29\end{array}$ $\begin{array}{lllllll}1424.96 & 1427.55 & 1427.91 & 1429.01 & 1430.66 & 1465.82\end{array}$ $\begin{array}{lllllll}1468.74 & 1469.02 & 1475.59 & 1482.04 & 1488.30 & 1493.67\end{array}$ $\begin{array}{lllllll}1496.28 & 1502.47 & 1503.51 & 1504.16 & 1505.83 & 1506.14\end{array}$ $\begin{array}{lllllll}1511.22 & 1514.98 & 1522.28 & 1523.09 & 1523.54 & 1524.59\end{array}$ $\begin{array}{lllllll}1526.14 & 1540.71 & 1542.41 & 1592.65 & 1626.38 & 1633.28\end{array}$ $\begin{array}{lllllll}1649.78 & 1654.00 & 1656.84 & 1658.41 & 1662.17 & 1677.18\end{array}$ $\begin{array}{lllllll}1683.39 & 3038.82 & 3040.42 & 3042.89 & 3043.14 & 3086.07\end{array}$ $\begin{array}{lllllll}3086.25 & 3095.95 & 3100.20 & 3101.82 & 3102.59 & 3105.68\end{array}$ $\begin{array}{lllllll}3110.85 & 3119.48 & 3122.07 & 3123.88 & 3126.17 & 3129.36\end{array}$ $\begin{array}{lllllll}3138.53 & 3163.66 & 3167.50 & 3171.58 & 3175.25 & 3182.49\end{array}$ $\begin{array}{llllll}3182.98 & 3187.11 & 3187.93 & 3188.05 & 3194.26 & 3195.48\end{array}$ $\begin{array}{llllll}3202.19 & 3205.54 & 3215.07 & 3220.96 & 3232.24 & 3247.95\end{array}$ $3249.27 \quad 3250.07 \quad 3488.77$

\footnotetext{
${ }^{3}$ bb-LA

$\begin{array}{llllll}11.24 & 26.43 & 27.39 & 30.32 & 39.87 & 41.41\end{array}$ $\begin{array}{lllllll}43.29 & 48.74 & 53.07 & 57.41 & 62.32 & 67.76\end{array}$ $\begin{array}{lllllll}70.85 & 78.39 & 81.14 & 95.02 & 110.63 & 114.94\end{array}$ $\begin{array}{llllll}124.52 & 125.14 & 139.46 & 146.11 & 155.59 & 171.17\end{array}$ $\begin{array}{llllll}176.90 & 178.46 & 182.28 & 196.41 & 207.14 & 209.42\end{array}$ $\begin{array}{llllll}213.68 & 223.53 & 229.36 & 231.46 & 238.99 & 241.85\end{array}$ $\begin{array}{llllll}247.04 & 255.33 & 259.92 & 274.18 & 280.61 & 306.58\end{array}$ $\begin{array}{lllllll}311.80 & 314.71 & 325.12 & 329.95 & 340.90 & 353.61\end{array}$ $\begin{array}{llllll}377.82 & 397.14 & 401.76 & 423.42 & 464.31 & 470.47\end{array}$ $\begin{array}{llllll}478.01 & 506.05 & 514.30 & 519.52 & 524.08 & 528.70\end{array}$ $\begin{array}{llllll}530.56 & 531.09 & 545.31 & 547.87 & 551.54 & 563.92\end{array}$ $\begin{array}{llllll}576.91 & 581.22 & 590.92 & 597.80 & 606.38 & 616.90\end{array}$ $\begin{array}{lllllll}625.61 & 628.25 & 640.88 & 652.05 & 667.94 & 687.49\end{array}$ $\begin{array}{lllllll}694.09 & 708.40 & 720.69 & 728.26 & 751.88 & 755.49\end{array}$ $\begin{array}{llllll}762.17 & 782.58 & 788.51 & 804.22 & 820.62 & 832.49\end{array}$ $\begin{array}{llllll}842.80 & 843.43 & 850.11 & 855.66 & 860.32 & 867.72\end{array}$ $\begin{array}{lllllll}876.57 & 888.49 & 893.33 & 895.93 & 908.25 & 911.56\end{array}$ $\begin{array}{lllllll}913.85 & 921.47 & 941.62 & 956.43 & 960.78 & 965.04\end{array}$ $\begin{array}{lllllll}974.48 & 976.24 & 984.90 & 997.17 & 1008.23 & 1009.51\end{array}$ $\begin{array}{lllllll}1014.16 & 1017.52 & 1022.98 & 1026.34 & 1039.29 & 1046.02\end{array}$ $\begin{array}{llllll}1046.97 & 1050.07 & 1055.56 & 1058.72 & 1060.94 & 1064.92\end{array}$ $\begin{array}{lllllll}1065.73 & 1066.34 & 1068.66 & 1093.50 & 1102.72 & 1114.36\end{array}$ $\begin{array}{lllllll}1124.73 & 1127.52 & 1155.87 & 1164.98 & 1172.67 & 1175.53\end{array}$ $\begin{array}{lllllll}1189.39 & 1193.03 & 1196.78 & 1203.31 & 1205.33 & 1212.65\end{array}$ $\begin{array}{lllllll}1218.13 & 1219.96 & 1225.13 & 1253.40 & 1261.62 & 1267.74\end{array}$ $\begin{array}{lllllll}1273.51 & 1300.49 & 1309.87 & 1314.66 & 1326.80 & 1331.10\end{array}$ $\begin{array}{llllll}1335.19 & 1337.46 & 1341.03 & 1349.18 & 1351.42 & 1365.63\end{array}$
}

$\begin{array}{llllll}1371.29 & 1373.17 & 1373.84 & 1381.39 & 1397.86 & 1410.60\end{array}$ $\begin{array}{llllll}1424.29 & 1426.96 & 1427.39 & 1428.14 & 1431.23 & 1443.97\end{array}$ $\begin{array}{lllllll}1456.30 & 1462.29 & 1466.90 & 1467.77 & 1474.29 & 1480.38\end{array}$ $\begin{array}{lllllll}1494.73 & 1496.71 & 1501.70 & 1503.44 & 1503.59 & 1504.52\end{array}$ $\begin{array}{lllllll}1507.59 & 1509.77 & 1514.80 & 1517.30 & 1521.76 & 1522.92\end{array}$ $\begin{array}{lllllll}1524.30 & 1526.50 & 1534.76 & 1543.59 & 1556.42 & 1589.69\end{array}$ $\begin{array}{lllllll}1607.07 & 1633.30 & 1643.07 & 1648.30 & 1654.15 & 1659.57\end{array}$ $\begin{array}{lllllll}1679.30 & 3039.77 & 3039.89 & 3043.11 & 3044.10 & 3073.06\end{array}$ $\begin{array}{lllllll}3081.08 & 3096.57 & 3101.66 & 3102.17 & 3103.09 & 3107.78\end{array}$ $\begin{array}{llllll}3111.88 & 3114.90 & 3126.00 & 3129.76 & 3130.31 & 3133.34\end{array}$ $\begin{array}{llllll}3139.66 & 3164.83 & 3166.46 & 3172.08 & 3173.54 & 3182.86\end{array}$ $\begin{array}{lllllll}3187.11 & 3189.00 & 3191.94 & 3192.70 & 3195.66 & 3208.13\end{array}$ $\begin{array}{llllll}3209.41 & 3213.70 & 3219.82 & 3226.38 & 3245.53 & 3247.35\end{array}$ $3248.82 \quad 3254.49 \quad 3490.83$

\footnotetext{
${ }^{1} \mathbf{4 c - L A}$

$\begin{array}{llllll}18.72 & 26.34 & 30.46 & 32.06 & 35.10 & 40.84\end{array}$ $\begin{array}{llllll}41.62 & 49.72 & 50.79 & 53.75 & 57.80 & 62.66\end{array}$ $\begin{array}{llllll}73.65 & 75.50 & 78.58 & 88.46 & 91.32 & 104.60\end{array}$ $\begin{array}{llllll}106.89 & 120.83 & 135.69 & 137.66 & 150.28 & 172.53\end{array}$ $\begin{array}{lllllll}176.11 & 185.63 & 198.84 & 208.77 & 212.17 & 220.72\end{array}$ $\begin{array}{llllll}222.98 & 229.00 & 235.36 & 237.73 & 240.96 & 242.56\end{array}$ $\begin{array}{llllll}248.18 & 253.90 & 272.83 & 278.00 & 288.87 & 297.64\end{array}$ $\begin{array}{llllll}307.15 & 311.03 & 326.57 & 331.31 & 340.25 & 351.95\end{array}$ $\begin{array}{lllllll}379.48 & 400.02 & 409.43 & 419.59 & 457.12 & 477.24\end{array}$ $\begin{array}{lllllll}491.53 & 501.87 & 519.16 & 523.32 & 526.64 & 527.33\end{array}$ $\begin{array}{lllllll}533.38 & 535.32 & 547.90 & 551.40 & 553.95 & 572.98\end{array}$ $\begin{array}{lllllll}580.39 & 586.74 & 602.27 & 608.16 & 617.30 & 625.41\end{array}$ $\begin{array}{lllllll}628.08 & 634.65 & 670.43 & 679.58 & 690.87 & 706.41\end{array}$ $\begin{array}{llllll}713.86 & 717.92 & 720.23 & 740.27 & 756.66 & 770.06\end{array}$ $\begin{array}{lllllll}782.17 & 787.47 & 825.48 & 843.80 & 849.54 & 850.87\end{array}$ $\begin{array}{lllllll}852.69 & 856.87 & 860.28 & 870.46 & 877.96 & 878.64\end{array}$ $\begin{array}{lllllll}890.33 & 909.37 & 910.41 & 910.94 & 922.24 & 940.83\end{array}$ $\begin{array}{llllll}941.41 & 950.63 & 959.59 & 960.60 & 966.02 & 976.14\end{array}$ $\begin{array}{llllll}985.07 & 1002.41 & 1008.85 & 1010.52 & 1013.56 & 1013.97\end{array}$ $\begin{array}{llllll}1018.25 & 1019.88 & 1022.62 & 1038.26 & 1045.17 & 1046.90\end{array}$ $\begin{array}{lllllll}1050.06 & 1053.57 & 1058.29 & 1061.89 & 1065.43 & 1065.85\end{array}$ $\begin{array}{llllll}1066.11 & 1067.42 & 1073.52 & 1088.50 & 1106.42 & 1121.04\end{array}$ $\begin{array}{llllll}1122.66 & 1131.16 & 1156.96 & 1172.95 & 1180.83 & 1192.04\end{array}$ $\begin{array}{lllllll}1196.12 & 1203.45 & 1204.47 & 1211.15 & 1216.58 & 1218.88\end{array}$ $\begin{array}{lllllll}1221.37 & 1233.98 & 1251.11 & 1257.74 & 1265.11 & 1276.20\end{array}$ $\begin{array}{lllllll}1290.07 & 1305.54 & 1315.23 & 1316.87 & 1327.03 & 1334.90\end{array}$ $\begin{array}{llllllll}1337.02 & 1338.38 & 1340.47 & 1349.70 & 1362.90 & 1367.20\end{array}$ $\begin{array}{lllllll}1371.34 & 1373.08 & 1375.67 & 1382.77 & 1399.66 & 1421.97\end{array}$ $\begin{array}{llllllll}1425.07 & 1425.77 & 1427.79 & 1430.87 & 1431.95 & 1466.21\end{array}$ $\begin{array}{lllllll}1469.09 & 1469.80 & 1475.67 & 1482.61 & 1487.46 & 1493.27\end{array}$ $\begin{array}{lllllll}1496.87 & 1498.81 & 1499.91 & 1502.60 & 1504.07 & 1506.33\end{array}$ $\begin{array}{lllllll}1514.06 & 1515.37 & 1521.99 & 1524.15 & 1524.54 & 1526.08\end{array}$ $\begin{array}{lllllll}1526.55 & 1541.35 & 1542.25 & 1586.40 & 1624.90 & 1633.50\end{array}$ $\begin{array}{llllll}1646.48 & 1654.08 & 1656.03 & 1657.29 & 1662.21 & 1680.25\end{array}$ $\begin{array}{lllllll}1683.28 & 3038.88 & 3042.57 & 3043.67 & 3044.25 & 3085.95\end{array}$ $\begin{array}{lllllll}3087.93 & 3095.46 & 3100.23 & 3102.17 & 3105.88 & 3107.63\end{array}$ $\begin{array}{lllllll}3111.13 & 3123.09 & 3125.65 & 3126.25 & 3129.23 & 3129.41\end{array}$ $\begin{array}{llllll}3138.29 & 3164.34 & 3170.83 & 3174.56 & 3176.79 & 3177.40\end{array}$ $\begin{array}{lllllll}3178.83 & 3183.30 & 3190.05 & 3194.66 & 3196.02 & 3196.96\end{array}$ $\begin{array}{llllll}3201.49 & 3205.21 & 3213.91 & 3221.04 & 3237.23 & 3244.79\end{array}$ $3247.68 \quad 3249.27 \quad 3485.39$

$====$
${ }^{3} \mathbf{4}-\mathbf{L A}$

$\begin{array}{llllll}15.20 & 23.66 & 29.34 & 35.08 & 36.03 & 44.70\end{array}$
} 
$\begin{array}{llllll}47.22 & 49.88 & 56.54 & 62.03 & 68.35 & 71.85\end{array}$

$\begin{array}{lllllll}73.85 & 87.95 & 97.82 & 103.54 & 104.55 & 117.28\end{array}$

$\begin{array}{llllll}123.72 & 125.63 & 140.32 & 144.59 & 160.54 & 167.84\end{array}$

$\begin{array}{llllll}177.48 & 181.79 & 196.67 & 204.21 & 207.33 & 216.39\end{array}$

$\begin{array}{llllll}223.64 & 231.07 & 236.24 & 240.57 & 242.23 & 247.97\end{array}$

$\begin{array}{llllll}251.68 & 258.07 & 270.83 & 279.56 & 295.63 & 305.67\end{array}$

$\begin{array}{llllll}313.58 & 322.11 & 324.04 & 331.68 & 339.83 & 349.39\end{array}$

$\begin{array}{llllll}376.64 & 400.04 & 401.01 & 422.60 & 462.66 & 473.84\end{array}$

$\begin{array}{lllllll}476.52 & 509.81 & 517.40 & 519.51 & 524.24 & 526.51\end{array}$

$\begin{array}{llllll}528.63 & 531.13 & 545.12 & 549.42 & 553.29 & 571.03\end{array}$

$\begin{array}{lllllll}573.37 & 578.99 & 586.39 & 593.58 & 607.82 & 617.19\end{array}$

$\begin{array}{llllll}627.02 & 631.74 & 648.69 & 656.06 & 672.79 & 691.12\end{array}$

$\begin{array}{llllll}699.96 & 704.03 & 716.55 & 727.68 & 748.65 & 756.51\end{array}$

$\begin{array}{lllllll}764.57 & 787.05 & 805.97 & 819.27 & 825.69 & 834.27\end{array}$

$\begin{array}{lllllll}838.09 & 843.27 & 849.16 & 856.00 & 863.20 & 868.64\end{array}$

$\begin{array}{llllll}875.19 & 895.29 & 899.35 & 905.10 & 909.45 & 913.53\end{array}$

$\begin{array}{llllll}934.06 & 939.78 & 951.25 & 957.97 & 962.69 & 966.80\end{array}$

$\begin{array}{lllllll}974.06 & 978.39 & 983.20 & 995.12 & 1007.12 & 1009.42\end{array}$

$\begin{array}{lllllll}1013.78 & 1017.49 & 1023.07 & 1025.23 & 1038.40 & 1046.49\end{array}$

$\begin{array}{lllllll}1047.14 & 1050.85 & 1055.53 & 1058.76 & 1059.69 & 1062.38\end{array}$

$\begin{array}{lllllll}1065.09 & 1065.42 & 1066.46 & 1077.82 & 1101.51 & 1111.97\end{array}$

$\begin{array}{lllllll}1125.03 & 1130.71 & 1155.65 & 1166.41 & 1172.98 & 1176.23\end{array}$

$\begin{array}{lllllll}1189.76 & 1194.04 & 1194.98 & 1203.21 & 1204.92 & 1211.83\end{array}$

$\begin{array}{llllllll}1214.63 & 1220.52 & 1222.25 & 1251.96 & 1259.90 & 1263.43\end{array}$

$\begin{array}{lllllll}1273.27 & 1295.98 & 1303.33 & 1309.05 & 1313.96 & 1328.80\end{array}$ $\begin{array}{lllllll}1334.41 & 1337.15 & 1339.96 & 1348.66 & 1356.95 & 1366.97\end{array}$ $\begin{array}{lllllll}1369.85 & 1371.58 & 1374.06 & 1380.71 & 1397.79 & 1412.84\end{array}$ $\begin{array}{lllllll}1425.17 & 1426.17 & 1428.28 & 1429.27 & 1431.40 & 1451.03\end{array}$ $\begin{array}{llllllll}1457.03 & 1463.07 & 1468.10 & 1469.40 & 1479.78 & 1480.80\end{array}$ $\begin{array}{lllllll}1494.14 & 1494.42 & 1496.85 & 1500.71 & 1503.93 & 1507.89\end{array}$ $\begin{array}{lllllll}1508.86 & 1512.15 & 1514.98 & 1518.80 & 1523.00 & 1523.62\end{array}$ $\begin{array}{lllllll}1524.86 & 1527.98 & 1535.58 & 1544.27 & 1557.52 & 1591.93\end{array}$ $\begin{array}{llllllll}1599.74 & 1633.56 & 1642.79 & 1648.06 & 1649.30 & 1654.69\end{array}$ $\begin{array}{lllllll}1679.83 & 3039.92 & 3041.30 & 3042.86 & 3046.84 & 3073.81\end{array}$ $\begin{array}{lllllll}3080.88 & 3096.05 & 3101.85 & 3102.86 & 3103.30 & 3108.86\end{array}$ $\begin{array}{lllllll}3112.77 & 3113.38 & 3125.47 & 3129.54 & 3130.35 & 3133.38\end{array}$ $\begin{array}{llllll}3140.05 & 3165.31 & 3166.68 & 3172.11 & 3183.67 & 3184.06\end{array}$ $\begin{array}{lllllll}3184.80 & 3191.55 & 3194.39 & 3195.65 & 3195.74 & 3196.55\end{array}$ $\begin{array}{llllll}3204.43 & 3217.75 & 3221.60 & 3223.77 & 3245.78 & 3246.03\end{array}$ $3247.78 \quad 3248.58 \quad 3488.36$ 


\section{References and Footnotes}

${ }^{1}$ Kim, Y.; Chang, S. Angew. Chem., Int. Ed. 2016, 55, 218-222.

${ }^{2}$ Kim, E.; Koh, M.; Lim, B. J.; Park, S. B. J. Am. Chem. Soc. 2011, 133, 6642-6649.

${ }^{3}$ El-Batta, A.; Jiang, C.; Zhao, W.; Anness, R.; Cooksy, A. L.; Bergdahl, M. J. Org. Chem. 2007, 72, 5244-5259.

${ }^{4}$ Ortega, V.; del Castillo, E.; Csákÿ, A. G. Org. Lett. 2017, 19, 6236-6239.

${ }^{5}$ Brimioulle, R.; Bach, T. Angew. Chem., Int. Ed. 2014, 53, 12921-12924.

${ }^{6}$ The weak ${ }^{13} \mathrm{C}$ signal was confirmed by HMBC.

${ }^{7}$ (E)-3-(4-Methoxyphenyl)acrylate was prepared according to the literature procedure: Ambler, B. R.; Altman, R. A. Org. Lett. 2013, 15, 5578-5581.

${ }^{8}$ Kamada, K.; Iwase, Y.; Sakai, K.; Kondo, K.; Ohta, K. J. Phys. Chem. C 2009, 113, $11469-$ 11474.

9 Miller, Z. D.; Lee, B. J.; Yoon, T. P. Angew. Chem., Int. Ed. 2017, 56, 11891-11895.

${ }^{10}$ Parr, R. G.; Weitao, Y. Density-Functional Theory of Atoms and Molecules; Oxford University Press, 1994

${ }^{11}$ Bochevarov, A. D.; Harder, E.; Hughes, T. F.; Greenwood, J. R.; Braden, D. A.; Philipp, D. M.; Rinaldo, D.; Halls, M. D.; Zhang, J.; Friesner, R. A. Int. J. Quantum Chem. 2013, 113, 21102142 .

${ }^{12}$ (a) Becke, A. D. J. Chem. Phys. 1993, 98, 1372-1377; (b) Lee, C.; Yang, W.; Parr, R. G. Phys. Rev. B 1988, 37, 785-789; (c) Grimme, S.; Antony, J.; Ehrlich, S.; Krieg, H. J. Chem. Phys. 2010, 132, 154104.

${ }^{13}$ Ditchfield, R.; Hehre, W. J.; Pople, J. A. J. Chem. Phys. 1971, 54, 724-728.

${ }^{14}$ (a) Hay, P. J.; Wadt, W. R. J. Chem. Phys. 1985, 82, 299-310; (b) Wadt, W. R.; Hay, P. J. J. Chem. Phys. 1985, 82, 284-298; (c) Hay, P. J.; Wadt, W. R. J. Chem. Phys. 1985, 82, 270-283.

${ }^{15}$ Dunning, T. H. J. Chem. Phys. 1989, 90, 1007-1023.

${ }^{16}$ (a) Marten, B.; Kim, K.; Cortis, C.; Friesner, R. A.; Murphy, R. B.; Ringnalda, M. N.; Sitkoff, D.; Honig, B. J. Phys. Chem. 1996, 100, 11775-11788; (b) Edinger, S. R.; Cortis, C.; Shenkin, P. S.; Friesner, R. A. J. Phys. Chem. B 1997, 101, 1190-1197; (c) Friedrichs, M.; Zhou, R.;

Edinger, S. R.; Friesner, R. A. J. Phys. Chem. B 1999, 103, 3057-3061. 